PREMIO TESI DI DOTTORATO

ISSN 2612-8039 (PRINT) | ISSN 2612-8020 (ONLINE)

$-77-$ 
PREMIO TESI DI DOTTORATO

Commissione giudicatrice, anno 2018

Vincenzo Varano, Presidente della Commissione

Tito Arecchi, Area Scientifica

Aldo Bompani, Area delle Scienze Sociali

Mario Caciagli, Area delle Scienze Sociali

Franco Cambi, Area Umanistica

Paolo Felli, Area Tecnologica

Giancarlo Garfagnini, Area Umanistica

Roberto Genesio, Area Tecnologica

Flavio Moroni, Area Biomedica

Adolfo Pazzagli, Area Biomedica

Giuliano Pinto, Area Umanistica

Vincenzo Schettino, Area Scientifica

Luca Uzielli, Area Tecnologica

Graziella Vescovini, Area Umanistica 


\author{
Andrea Sacchetti
}

\title{
La costituente libertaria di Camillo Berneri
}

Un disegno politico tra federalismo e anarchismo

Firenze University Press

2019

Andrea Sacchetti, La costituente libertaria di Camillo Berneri. Un disegno politico tra federalismo e anarchismo, (C) 2020 Author(s), content CC BY 4.0 International, metadata CC0 1.0 Universal, published by Firenze University Press (www.fupress.com), ISSN 26128020 (online), ISBN 978-88-6453-991-1 (online PDF) 


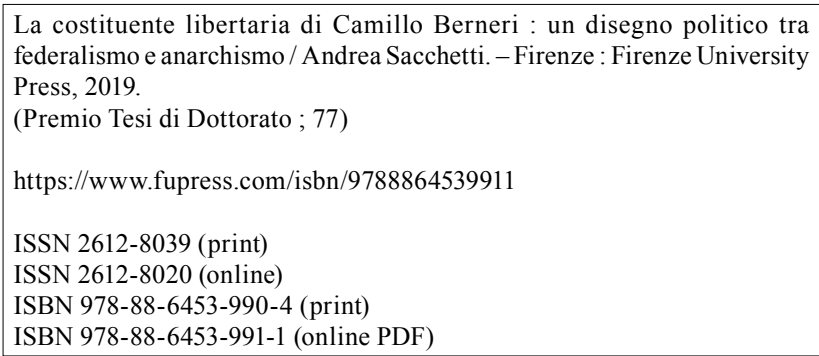

Graphic design: Alberto Pizarro Fernández, Lettera Meccanica SRLs

Peer Review Process

All publications are submitted to an external refereeing process under the responsibility of the FUP Editorial Board and the Scientific Committees of the individual series. The works published in the FUP catalogue are evaluated and approved by the Editorial Board of the publishing house. For a more detailed description of the refereeing process we refer to the official documents published on the website and in the online catalogue (www.fupress.com).

Firenze University Press Editorial Board

M. Garzaniti (Editor-in-Chief), M.E. Alberti, M. Boddi, A. Bucelli, R. Casalbuoni, A. Dolfi, R. Ferrise, M.C. Grisolia, P. Guarnieri, R. Lanfredini, P. Lo Nostro, G. Mari, A. Mariani, P.M. Mariano, S. Marinai, R. Minuti, P. Nanni, A. Orlandi, A. Perulli, G. Pratesi.

The online digital edition is published in Open Access on www.fupress.com.

Content license: the present work is released under Creative Commons Attribution 4.0 International license (CC BY 4.0: http://creativecommons.org/licenses/by/4.0/legalcode). This license allows you to share any part of the work by any means and format, modify it for any purpose, including commercial, as long as appropriate credit is given to the author, any changes made to the work are indicated and a URL link is provided to the license.

Metadata license: all the metadata are released under the Public Domain Dedication license (CC0 1.0 Universal: https://creativecommons.org/publicdomain/zero/1.0/legalcode).

(C) 2019 Author(s)

Published by Firenze University Press

Firenze University Press

Università degli Studi di Firenze

via Cittadella, 7, 50144 Firenze, Italy

www.fupress.com

This book is printed on acid-free paper

Printed in Italy 
A Lea, per tutto quello che ha chiesto per tutto quello che ha dato 



\section{Indice}

$\begin{array}{ll}\text { Elenco delle abbreviazioni } & 9\end{array}$

$\begin{array}{ll}\text { Introduzione } & 11\end{array}$

\section{Capitolo 1}

Il sentimento e l'impegno: la gioventù e la formazione politica di

Camillo Berneri

1. Come un giovane socialista divenne anarchico

2. Un giovane anarchico tra guerra e rivoluzione

\section{Capitolo 2}

Il federalismo per realizzare l'avvenire anarchico: la maturazione del pensiero politico di Camillo Berneri

1. Gli studi universitari e l'incontro con Salvemini 51

2. Il federalismo libertario per rinnovare l'anarchismo $\quad 73$

3. Programma minimo federalista, un pratico proposito 94

\section{Capitolo 3}

L'esilio senza requie e la costituente libertaria $\quad 119$

1. «L'anarchico più espulso d'Europa»: l'elaborazione politica 119

2. Per un'Italia federalista e libertaria: la Costituente 144

3. Sviluppi della riflessione e confronto con il «socialismo federalista liberale» 171

4. Verso un epilogo: ultimi episodi di riflessione federalista 190

$\begin{array}{ll}\text { Bibliografia } & 207\end{array}$

$\begin{array}{ll}\text { Indice dei nomi } & 227\end{array}$ 



\section{Elenco delle abbreviazioni}

ACS

AFB

AGL

AGS

ASUF

b.

c., cc.

cass.

$\mathrm{CPC}$
Archivio Centrale dello Stato

Archivio Famiglia Berneri-Aurelio Chessa

Archivi di Giustizia e Libertà

Archivio Gaetano Salvemini

Archivio Storico dell'Università degli Studi di Firence

busta

carta, carte

cassetta

Casellario Politico Centrale

Direzione Generale Pubblica Sicurezza, Affari Generali

Riservati

Dir. Gen. PS, Div. Pol. Polit. Direzione Generale Pubblica Sicurezza, Divisione

Polizia Politica

fsc.

fascicolo

ISRT

Istituto Storico della Resistenza in Toscana

Min. In.

Ministero dell'Interno

s. d.

senza data

s. 1 .

senza luogo

vol.

volume 



\section{Introduzione}

Art. 1 - L'Italia è una repubblica federale tendente a realizzare il massimo possibile di libertà e di giustizia. I suoi organi amministrativi, politici e giuridici emanano dal popolo, che ne controlla il funzionamento. La Repubblica è il complesso degli organi nazionali, regionali e municipali ${ }^{1}$.

Con questo primo articolo si apre il progetto di carta costituzionale scritto nell'ottobre 1935 dall'anarchico Berneri a Sartrouville, Parigi, durante il Convegno d'intesa degli anarchici italiani emigrati in Europa. Il testo di questa costituzione è stato reso noto e pubblicato soltanto nel 2001, probabilmente perché fino ad allora considerato troppo "eretico" e finanche imbarazzante rispetto all'immagine tipica di Berneri, noto sì per essere stato un spirito critico, ma ancor di più per essere stato uno dei più appassionati e preparati agitatori anarchici di inizio Novecento, nonché per essere diventato il martire della causa nella Spagna della Guerra Civile (193637). La moglie Giovanna Caleffi custodisce il documento in gran segreto e altrettanto farà, una volta divenuto curatore delle carte di Berneri, Aurelio Chessa. Solo nel 1977, in occasione del cinquantenario della morte, egli avvia una prima discussione per corrispondenza sull'opportunità o meno di rendere pubblico il documento, ma l'ipotesi di sottrarre questa "costituente libertaria" all'oscurità viene accantonata ${ }^{2}$.

Il nostro lavoro cerca di individuare le motivazioni storiche, culturali, politiche e strategiche che hanno condotto un anarchico come Berneri a redigere questo progetto, senza dubbio originale e senza precedenti all'interno del movimento anarchico. Nonostante siano passati più di quindici anni dalla sua pubblicazione, la Costituzione di Berneri non ha a nostro parere ancora ricevuto l'attenzione che meriterebbe; mancano soprattutto all'interno della storia del pensiero politico

${ }^{1}$ C. Berneri, Costituzione della Federazione Italiana Comuni Socialisti (F.I.C.S.), in Id., Anarchia e società aperta. Scritti editi ed inediti, a cura di Pietro Adamo, M\&B Publishing, Milano, 2001, p. 207. Nel documento originale la forma di governo era una «repubblica federale libertaria», ma quest'ultimo aggettivo è stato poi cancellato.

${ }^{2}$ La complessa vicenda è stata ricostruita da Pietro Adamo nella nota introduttiva al testo, vedi C. Berneri, Costituzione cit., pp. 205-206. 
contributi e studi che ne evidenzino l'innovatività e che rendano ragione della sua esistenza all'interno del percorso intellettuale berneriano, un percorso che ha tutto l'aspetto di una vera e propria battaglia di aggiornamento portata all'interno del movimento anarchico e tesa a mettere da parte molti principi teorici e a spingersi sul terreno del pratico e del contingente. È nostra opinione che questo documento rappresenti per Berneri un vero e proprio punto d'arrivo, magari non definitivo e senz'altro passibile di ulteriori modifiche, ma nondimeno un traguardo, un farsi finalmente concreto e nero su bianco di un progetto e di un auspicio di lunga gestazione in cui al movimento anarchico sarebbe toccato un ruolo da protagonista nel gettare le basi e nel realizzare una società di liberi ed eguali.

\section{Luigi Camillo Berneri}

Alto un metro e sessantotto, dalla corporatura snella. Capelli castani scuri, lisci e folti, viso pallido e lungo, fronte spaziosa e «sveggente», sopracciglia arcuate e castane, occhi ovali, grandi e neri, naso rettilineo e «grossetto», orecchie grandi, mento ovale, collo lungo e magro. Spalle leggermente curve, gambe dritte, «simpatico» di espressione fisionomica e «decente» nell'abbigliamento abituale. Così si legge aprendo il fascicolo della Prefettura di Reggio Emilia che descrive Camillo Berneri (Lodi, 1897 - Barcellona, 1937) ${ }^{3}$. Luigi Camillo Berneri ${ }^{4}$, questo il suo nome per esteso, nasce a Lodi dal padre Stefano e dalla madre Adalgisa Fochi, ma della città lombarda conserverà solamente il dato anagrafico del luogo di nascita: la sua è un'infanzia itinerante, segnata dai frequenti spostamenti della madre, maestra elementare. È in Emilia Romagna che Berneri troverà una prima residenza più stabile ed è qui che avvengono i suoi primi passi in politica, col l'adesione alla Federazione Giovanile Socialista di Reggio Emilia nel 1912. Vi milita appassionatamente finché, nel 1915, non avviene la svolta decisiva: rassegnate le dimissioni dalla Federazione Giovanile, Berneri aderisce all'anarchismo e a questo movimento e a questa idea politica resterà legato fino alla fine della sua breve esistenza.

Nel 1919, dopo il fuoco e il piombo della Grande Guerra, si trasferisce a Firenze, dove compie gli studi universitari presso la Facoltà di Filosofia e Filologia del Regio Istituto di Studi Superiori. In questo periodo avvengono due incontri fondamentali per lui, da un lato col professore di Storia Moderna Gaetano Salvemini

${ }^{3}$ Prefettura di Reggio Emilia, Connotati, in ACS, Min. In., CPC, Berneri Camillo, b. 537, fsc. I.

${ }^{4}$ Per maggiori dettagli biografici si rimanda a C. De Maria, Camillo Berneri. Tra anarchismo e liberalismo, Franco Angeli, Milano, 2004, pp. 15-113, e S. D'Errico, Anarchismo e politica. Nel problemismo e nella critica dell'anarchismo del ventesimo secolo, il "programma minimo" dei libertari del terzo millennio. Rilettura antologica e biografica di Camillo Berneri, Mimesis, Milano, 2007, pp. 497-617. Vedi anche la voce «Berneri, Camillo», in M. Antonioli et al. (diretto da), Dizionario Biografico degli anarchici italiani, vol I., Edizioni Biblioteca Franco Serantini, Pisa, 2003, pp. 142-149, e F. Madrid Santos, Camillo Berneri, un anarchico italiano (1897-1937). Rivoluzione e controrivoluzione in Europa (1917-1937), Edizioni Archivio Famiglia Berneri, Pistoia, 1985. 
con cui nel 1922 discuterà anche la sua tesi di laurea in Pedagogia sulla campagna dei clericali piemontesi per la libertà della scuola dal 1848 al 1859, dall'altro con l'anziano e autorevole militante anarchico Errico Malatesta con cui collabora, tra l'altro, alla redazione del quotidiano «Umanità Nova». Durante e successivamente alla sua formazione universitaria Berneri offre importanti contributi giornalistici alla stampa anarchica, ma anche a riviste di altra area politica quali, ad esempio, «L'Unità» di Gaetano Salvemini e «Rivoluzione Liberale» di Piero Gobetti. Il contatto col professore pugliese lo porta inoltre a stringere amicizia, tra gli altri, con i fratelli Rosselli ed Ernesto Rossi nell'ambito del Circolo di Cultura di Firenze.

Divenuto Professore di Filosofia, dal 1923 inizia a spostarsi tra diverse scuole tornando frequentemente nel capoluogo toscano dove mantiene i contatti con l'antifascismo locale; vanno ricordati a tal proposito la sua militanza nell'«Italia Libera», prima associazione antifascista che univa, tra gli altri, i fratelli Rosselli, Pietro Gobetti ed Ernesto Rossi, e il suo contributo alla diffusione del «Non Mollare» (1925), primo foglio clandestino contro il regime stampato a Firenze e animato da Salvemini, dai fratelli Rosselli e da Piero Calamandrei. Perseguitato dal regime fascista, Berneri è costretto a emigrare in Francia nell'aprile 1926 dove ritorna a collaborare con la stampa anarchica e prende contatto col movimento anarchico internazionale. Caduto nella rete di provocazione fascista, viene espulso dalla Francia alla fine del 1928 e trascorre un periodo travagliato nei sette anni successivi tra arresti, revoche e proroghe del permesso di soggiorno e relative espulsioni, spostandosi fra Belgio, Lussemburgo, Paesi Bassi, Germania e Svizzera; otterrà il permesso di soggiorno in Francia soltanto nel $1935^{5}$.

Il 29 luglio 1936 decide di passare clandestinamente la frontiera spagnola per prendere parte alla Guerra Civile ${ }^{6}$, arruolandosi nelle milizie organizzate dalla sigla d'ispirazione anarcosindacalista della Confederación Nacional del Trabajo (CNT), appoggiata dalla Federación Anarquista Ibérica (FAI), cui era storicamente legata. Berneri lavorerà alla costituzione di una sezione italiana della colonna Ascaso, il cui patto d'intesa viene siglato il 17 agosto 1936 con Carlo Rosselli e il repubblicano Mario Angeloni. Il 5 maggio 1937 Berneri viene assassinato da poliziotti agli ordini di ufficiali di parte stalinista, nel contesto degli scontri di Barcellona che oppongono appunto le formazioni del filosovietico Partit Socialista Unificat de Catalunya (PSUC) a quelle della CNT-FAI e del Patrido Obrero de Unificación Marxista (POUM), di ispirazione marxista-leninista ma antistalinista ${ }^{7}$.

${ }^{5}$ La travagliata vicenda di questi anni è stata ben ricostruita da C. De Maria, Camillo Berneri cit., pp. 31-86, e da S. D'Errico, Anarchismo e politica cit., pp. 554-570.

${ }^{6}$ Sulla guerra civile spagnola risultano ancora indispensabili N. Torcellan, Gli italiani in Spagna: bibliografia della guerra civile spagnola, Franco Angeli, Milano, 1988, e H. Thomas, Storia della guerra civile spagnola, Einaudi, Torino, 1963; tra gli studi più recenti si vedano almeno L. Ceva, Spagne 1936-1939: politica e guerra civile, Franco Angeli, Milano, 2010, B. Bennassar, La guerra di Spagna: una tragedia nazionale, Einaudi, Torino, 2006, e G. Ranzato, L'eclissi della democrazia: la guerra civile spagnola e le sue origini (19311939), Bollati Boringhieri, Torino, 2003. 


\section{Gli studi su Berneri}

Attualmente le ricerche e i lavori intorno alla figura e al pensiero di Camillo Berneri appaiono numericamente non esigui, ma complessivamente poco noti o diffusi tra gli storici del pensiero politico. A partire dalla sua morte, il solo movimento anarchico ne ha a lungo conservato la memoria postuma e non di rado ciò ha condotto al prevalere di una tendenza più memorialistica che storica.

Il primo tentativo di inquadramento critico si ha negli anni '60 del Novecento con la pubblicazione di una raccolta di scritti curata da Pier Carlo Masini e Alberto Sorti: la tendenza prevalente di questa storiografia è stata soprattutto quella di enfatizzare l'opposizione di Berneri ai totalitarismi, il ruolo organizzativo e pubblicistico ricoperto in Spagna, nonché il suo "martirio"8. Nel decennio successivo, inizia a manifestarsi un interesse più profondo e un approccio più complesso alla figura dell'anarchico lodigiano nel contesto del Convegno di studi promosso in occasione del quarantennale della sua morte e tenutosi a Milano il 9 ottobre 1977, cui contribuiscono, tra gli altri, Pier Carlo Masini, Gino Cerrito, Giampietro Berti, Giovanbattista Carrozza e Umberto Marzocchi ${ }^{9}$.

Negli anni '80 inizia l'importante attività editoriale dell'Archivio Famiglia Berneri (curato e gestito a partire dal 1962 da Aurelio Chessa) ${ }^{10}$ che avvia la pubblicazione di numerosi scritti di Berneri: particolarmente importanti e significativi sono i due volumi dell'epistolario dell'anarchico lodigiano, usciti rispettivamente nel 1980 e nel 1984, e il primo studio storico sulla sua vita, il suo pensiero e la sua azione, di Francisco Madrid Santos, nel $1985^{11}$.

Studi più recenti, anche se spesso prossimi all'area anarchica, hanno contribuito a far emergere ancora di più la complessità del pensiero politico berneriano, le

${ }^{7}$ Per l'assassinio di Berneri si rimanda a S. D'Errico, Anarchismo e politica, cit., pp. 598-604, e a C. Venza, Tra rivoluzione e guerra. Libertari italiani nella Spagna degli anni Trenta, in La Resistenza sconosciuta. Gli anarchici e la lotta contro il fascismo, Milano, Zero in condotta, 2005, pp. 133-137. Recentemente una nuova e documentata ricerca sui moventi del delitto ha modificato la canonica ricostruzione fin qui accettata, vedi S. W. Pechar, Il caso Berneri. Antifascisti italiani nella Spagna rivoluzionaria (1936-1937), Edizioni ANPPIA, Roma, 2017.

${ }^{8}$ Vedi C. Berneri, Pietrogrado 1917 Barcellona 1937. Scritti scelti, a cura di Pier Carlo Masini e Alberto Sorti, Sugar, Milano 1964.

${ }^{9}$ Atti del Convegno di studi su Camillo Berneri, Milano, 9 ottobre 1977, La Cooperativa Tipografica Editrice, Carrara, 1979.

${ }^{10}$ Per una storia dell'archivio si rimanda alla scheda Archivio «Famiglia Berneri-Aurelio Chessa» a cura della Biblioteca Panizzi di Reggio Emilia $<$ http://panizzi.comune.re.it/allegati/ABC completo.pdf $>$ (09/2019).

${ }^{11}$ Vedi C. Berneri, Epistolario inedito, vol. I, a cura di Aurelio Chessa e Pier Carlo Masini, Edizioni Archivio Famiglia Berneri, Pistoia, 1980 e Id., Epistolario inedito, vol. II, a cura di Luigi Di Lembo e Paola Feri, Edizioni Archivio Famiglia Berneri, Pistoia, 1984; F. Madrid Santos, Camillo Berneri cit. Si veda inoltre Memoria antologica, saggi critici e appunti biografici in ricordo di Camillo Berneri nel cinquantesimo della morte, Edizioni Archivio Famiglia Berneri, Pistoia, 1986. 
critiche che Berneri ha portato al movimento anarchico e la sua proposta di aggiornarne e di attualizzarne la battaglia; si tratta in particolare delle ricerche di Giampietro Berti, che nella sua storia del pensiero anarchico ha dedicato ampio spazio alla figura dell'anarchico lodigiano, di Pietro Adamo e di Carlo De Maria, che hanno soprattutto sottolineato gli elementi di maggiore eccentricità di Berneri rispetto al movimento anarchico, e infine di Stefano D'Errico, che ha offerto un'ampia rilettura antologia e biografica con l'obiettivo di convincere anarchici e libertari a riappropriarsi dell'eredità del pensiero e delle battaglie berneriane ${ }^{12}$. La collaborazione tra Archivio Famiglia Berneri-Aurelio Chessa e Biblioteca Panizzi ha portato nel corso degli anni Duemila alla pubblicazione degli atti di due giornate di studi - svoltesi a Reggio Emilia nel 2005 e ad Arezzo nel 2007 - che hanno ulteriormente contribuito ad aumentare la ricchezza e la complessità degli studi critici su Camillo Berneri ${ }^{13}$.

Infine, vanno segnalati i recenti lavori di Claudio Strambi, che tentano di ricostruire la biografia, il pensiero e l'azione di Berneri collocandole all'interno della complessa e articolata storia del movimento anarchico italiano. La voce e le idee berneriane vengono così collocate in una precisa cornice storica, economica e sociale e, al contempo, vengono situate all'interno di un coro plurale e ben più vasto $^{14}$.

\section{Il federalismo di Berneri e il rapporto con Salvemini}

Il presente lavoro si propone di indagare un aspetto del pensiero politico di Berneri su cui poco è stato scritto o detto, vale a dire le sue idee federaliste ${ }^{15}$, a loro

\footnotetext{
${ }^{12}$ Vedi il capitolo dedicato a Berneri in G. Berti, Il pensiero anarchico. Dal Settecento al Novecento, Lacaita, Manduria-Bari-Roma, 1998, pp. 857-893; quindi l'antologia di scritti editi e inediti C. Berneri, Anarchia e società aperta cit., il lavoro di C. De Maria, Camillo Berneri cit., e il volume di S. D'Errico, Anarchismo e politica cit. Da segnalare inoltre G. Cerrito, Introduzione a C. Berneri, Scritti scelti, Zero in condotta, Milano, 2013, pp. 13-41, e il recente C. De Maria, Una famiglia anarchica. La vita dei Berneri tra affetti, impegno ed esilio nell'Europa del Novecento, Viella, Roma, 2019, che ricostruisce la vicenda biografica e il pensiero politico di Camillo Berneri alla luce dei suoi legami privati e pubblici.

${ }^{13}$ Camillo Berneri, singolare/plurale. Atti della giornata di studi, Reggio Emilia, 28 maggio 2005, Edizioni Biblioteca Panizzi e Archivio Famiglia Berneri-Aurelio Chessa, Reggio Emilia, 2007 e G. Berti, G. Sacchetti (a cura di), Un libertario in Europa. Camillo Berneri: fra totalitarismi e democrazia. Atti del convegno di studi storici, Arezzo, 5 maggio 2007, Edizioni Biblioteca Panizzi e Archivio Famiglia Berneri-Aurelio Chessa, Reggio Emilia, 2010.

${ }^{14} \mathrm{C}$. Strambi, L'inquieta attitudine: Camillo Berneri e la vicenda politica dell'anarchismo in Italia. Primo Libretto, Edizioni Kronstadt, Pisa, 2015, e Id., , L'inquieta attitudine: Camillo Berneri e la vicenda politica dell'anarchismo in Italia. Secondo Libro, "Il biennio rosso e rossonero", Edizioni Kronstadt, Pisa, 2017.

${ }^{15}$ Ad oggi si hanno solo i paragrafi dedicati a Berneri nello studio di L. Di Lembo, Il federalismo libertario e anarchico in Italia. Dal Risorgimento alla Seconda Guerra Mondiale, Edizioni Sempre Avanti!, Livorno, 1994, in particolare le pp. 44-58, e C. Berneri,
} 
volta complesse e caratterizzate dalla presenza di diverse influenze, tra cui quelle di «Cattaneo completato da Salvemini e dal sovietismo»" ${ }^{16}$. A questo scopo ci proponiamo di mettere in luce la maturazione delle idee del Berneri intorno al federalismo libertario, aspetto che riteniamo molto importante e significativo per comprendere i suoi tentativi di rinnovamento dell'anarchismo in «una sintesi che tende a mettere insieme i principi supremi del liberalismo e del socialismo (libertà individuale e uguaglianza sociale), con il metodo offerto dai modelli del comunalismo, del federalismo, del sovietismo e del sindacalismo», come hanno correttamente indicato i curatori Berti e Sacchetti nell'introduzione a Un libertario in Europa, ultima raccolta di atti di convegno sull'anarchico lodigiano pubblicata, anche se poi il tema del federalismo non viene ulteriormente approfondito ${ }^{17}$. Tale "sintesi" doveva essere funzionale a trovare sbocchi di realizzabilità pratica per la battaglia di libertà dell'anarchismo, una necessità avvertita con forza dall'anarchico lodigiano fin dagli anni dei suoi studi universitari alla scuola di Salvemini.

Il rapporto col professore pugliese è il secondo elemento che ha destato la nostra attenzione e che ci ha portato a condurre buona parte della ricerca sul rapporto e sullo scambio avvenuto tra $\mathrm{i}$ due. È emerso in particolare non solo che la tendenza berneriana di portare la battaglia dell'anarchismo su un terreno maggiormente pratico e contingente si sia sviluppata proprio in quegli anni e a contatto con la lezione concretista del professore pugliese, ma anche che l'attenzione e lo studio profondo che il Berneri porterà sul tema del federalismo abbia avuto il suo inizio proprio sulla scorta di letture e suggestioni salveminiane. Un altro aspetto si è fatto dunque largo, ossia che l'apporto e l'influenza di Salvemini su Berneri studente e militante siano stati molto più profondi e decisivi di quanto gli studi critici sull'anarchico lodigiano abbiano finora mostrato: se il nome di Salvemini e il riconoscimento della sua importanza invero non mancano, è anche vero che nessuno ha sinora pensato di sondare e indagare più a fondo la natura e i caratteri di questo apporto. Abbiamo dunque indirizzato le nostre ricerche e le nostre osservazioni in questo senso, mettendo a confronto la produzione di Berneri con quella di Salvemini, cogliendo citazioni, rimandi e influenze che ci sembrano evidenti e che sono state sorprendentemente ignorate fin qui.

L'anarchico lodigiano, come già il suo professore prima di lui, svolge una battaglia per abbandonare l'attaccamento a formule e ad astrattezze ideologiche e per avviare lo studio dei problemi sociali, economici e politici attraverso cui elaborare soluzioni concrete e praticabili, privilegiando la tattica degli obiettivi minimi rispetto alla pretesa di veder realizzare immediatamente le proprie aspettative massime. Questo atteggiamento intellettuale viene riletto da Berneri in chiave rivoluzionaria e

Il federalismo libertario, a cura di Patrizio Mauti, La Fiaccola, Ragusa, 1992, una raccolta di testi e saggi berneriani inerenti appunto al federalismo introdotta da uno schematico inquadramento critico.

${ }^{16}$ Lettera di Camillo Berneri a Libero Battistelli (s.l., s.d., ma probabilmente 1929), in C. Berneri, Epistolario inedito, vol. I cit., p. 19.

${ }^{17}$ G. Berti, G. Sacchetti, Introduzione a Un libertario in Europa cit., p. 12. 
agitato con coerenza all'interno del movimento anarchico a partire dall'immediato dopoguerra e fino alla fine dei suoi giorni.

\section{Il progetto di Costituzione}

Tale atteggiamento emerge e si precisa già nel corso degli anni '20, ma è soprattutto durante il periodo del fuoruscitismo degli anni '30 che il lodigiano torna a insistervi: gli anarchici rischiavano in quel frangente di rimanere relegati ai margini del movimento antifascista e incapaci di incidervi, proprio per via della loro scarsa disposizione a porsi il problema di obiettivi rivoluzionari attuabili. Si può dire che Camillo Berneri sia stato il più attivo - se non addirittura un caso isolato - da questo punto di vista, nel ricercare contatti con gli elementi antifascisti più dinamici e disponibili a muoversi all'interno di coordinate di tipo libertario, da lui individuati in particolare nei repubblicani di sinistra, nel movimento di «Giustizia e Libertà», nei socialisti e nei comunisti dissidenti ${ }^{18}$. La relazione da lui presentata durante il Convegno d'intesa degli anarchici italiani di Sartrouville (ottobre 1935) è in questo senso molto interessante, in quanto porterà alla redazione, a margine, della Costituzione della Federazione Italiana Comuni Socialisti (F.I.C.S.): un documento fondativo senza precedenti per l'area anarchica e che doveva avere probabilmente il fine di avviare un confronto e un dibattito con altre forze politiche sensibili a contenuti libertari e federalisti.

Pubblicato per la prima volta, come si è detto, nel 2001 nell'antologia di scritti berneriani curata da Pietro Adamo, ci sembra che questo progetto costituzionale non abbia ancora goduto del necessario interesse negli studi della storia delle dottrine politiche. Invece un simile scritto è senza dubbio, in termini generali, qualcosa di innovativo e degno di attenzione già solo per il fatto di essere stato redatto da un anarchico; nel caso specifico, lo è in quanto rappresenta un originale tentativo di dare un programma e di organizzare e riunire in un polo tutte quelle forze d'ispirazione socialista che rifiutavano l'impostazione marxista, in quanto centralista e autoritaria. Berneri tenta in questo modo di tracciare una sorta di programma di minima il più concreto possibile per un socialismo 'altro', che guardasse all'autonomia come dimensione virtuosa, entro cui sviluppare le libertà individuali e le capacità progressiste della società. Come sintetizza efficacemente D'Errico, egli decide di «portare l'anarchismo dalla protesta alla proposta» ${ }^{19}$, ritenendo prioritario dare una direzione pratica e attuabile dei suoi principi; ciò per evitare l'emarginazione politica degli anarchici e, con loro, dei fautori di una realtà sociale che rappresentasse un possibile sviluppo delle istanze democratiche e libertarie.

Questo lavoro si propone di ricostruire il percorso di Berneri ponendo particolare attenzione alle sue idee federaliste e alle sue proposte operative, con l'intento di gettare nuova luce e ampliare la prospettiva su un personaggio e un progetto politico ancora poco noti o comunque poco studiati dal punto di vista della Storia delle dottrine politiche.

\footnotetext{
${ }^{18} \mathrm{Su}$ Berneri e le alleanze si veda G. Sacchetti, Gli anarchici italiani e la questione delle alleanze, in Camillo Berneri, singolare/plurale cit., pp. 84-87.

${ }^{19}$ Stefano D'Errico, Anarchismo e politica cit., p. 396.
} 
Nel primo capitolo viene ripercorsa la gioventù e la formazione politica di Camillo Berneri, a partire dalla sua militanza all'interno della Federazione Giovanile Socialista, illustrando le ragioni che lo portano infine ad aderire al movimento anarchico. Particolare attenzione viene dedicata al suo impegno nel promuovere e diffondere la cultura nel proletariato, ma soprattutto alla sua vivace propaganda antimilitarista e rivoluzionaria, senza dubbio la motivazione principale che lo porterà lontano dai suoi giovani compagni socialisti.

Nel secondo capitolo vengono messe in luce le caratteristiche dell'incontro e del primo scambio intellettuale con il professor Salvemini e il parallelo emergere di un atteggiamento intellettuale che, pur senza perdere un certo slancio volontaristico, inizia a concentrarsi sullo studio e l'individuazione di problemi e aspetti critici di cui anche la rivoluzione libertaria deve farsi carico. È sempre in questo momento che Berneri, alla ricerca della possibilità di un'applicazione concreta dei principi anarchici, inizia su influenza salveminiana la sua riflessione sul federalismo quale sistema politico che possa aprire la strada verso una realizzazione graduale dell'abolizione dello Stato teorizzata dal suo movimento. Emergeranno anche i primi attriti e le prime polemiche con il movimento anarchico, incapace di giocare un ruolo da protagonista durante $\mathrm{i}$ fermenti rivoluzionari del dopoguerra e progressivamente ridotto al silenzio dopo l'imporsi del regime fascista.

Il terzo capitolo analizza quella che è l'ultima fase della riflessione federalista di Berneri, vale a dire quella dell'esilio in Francia. Il lodigiano tenta in questo periodo di contribuire alla ricomposizione e riorganizzazione del frammentato movimento anarchico, continuando a insistere sulla necessità di darsi un programma più concreto e attuabile, capace di aprirsi anche ad altre forze politiche affini e con cui sia possibile condividere alcuni punti. Il federalismo è ancora uno dei temi che Berneri affronta con maggiore frequenza, tanto da diventare l'idea-cardine su cui promuovere un'alleanza tra forze antifasciste contrarie all'accentramento del potere nelle mani dello Stato e decise a valorizzare le autonomie nella futura Italia liberata. Per evitare che gli anarchici si trovino nuovamente in condizione subalterna rispetto ad altri partiti o movimenti politici rivoluzionari, Berneri decide infine di partire da un documento fondativo provvisorio - la Costituzione - in modo da avere le basi e le linee generali di una piattaforma di azione comune in cui siano previste numerose garanzie atte a indebolire fortemente le attribuzioni dello Stato, con la prospettiva di poter nel tempo lavorare per ridurle ulteriormente e di aumentare per contro le autonomie individuali e sociali. Il contatto tra anarchici e giellisti e il dibattito tra Berneri e Carlo Rosselli assumono, da questo punto di vista, un grande significato e mostrano abbastanza chiaramente quali fossero i confini dell'azione che l'anarchico lodigiano intendesse operare.

Il lavoro si interrompe nel periodo compreso tra 1935 e 1936: sono gli ultimi momenti significativi di elaborazione politica, che prosegue in maniera episodica anche dopo che Berneri è accorso in Spagna per prendere parte alla difesa dell'esperienza repubblicana dalla reazione fascista; essendosi modificato sensibilmente lo scenario politico, l'urgenza dell'azione pone in secondo piano la questione del federalismo, in generale, e del progetto di costituzione in particolare. 
Le energie intellettuali e fisiche di Berneri prediligono in questo frangente l'analisi della situazione rivoluzionaria spagnola e dello scenario politico internazionale, $\mathrm{i}$ progetti per l'azione antifascista in Italia sono rimandati al profilarsi di situazioni maggiormente propizie, che forse proprio l'evolversi della situazione in Spagna avrebbe infine favorito. Berneri, come detto, troverà la morte a Barcellona e i suoi progetti federalisti libertari e le sue intuizioni programmatiche spireranno assieme a lui.

\section{Il federalismo libertario}

Lo studio del federalismo libertario ci ha posto però di fronte a un ulteriore dilemma: capire cosa si potesse e si dovesse intendere con questo termine e quali fossero i confini di tale paradigma politico. Dare un profilo netto del federalismo libertario è un'operazione complessa e la sensazione che si ha nel trattare tale argomento è quella di trovarsi di fronte a una sorta di "vuoto storiografico". Ovviamente non mancano cenni e riferimenti alle idee federali dei protagonisti dell'anarchismo nelle storie generali sul movimento, nonché una certa attenzione rivolta alle strutture organizzative che il movimento si è dato - strutture, per l'appunto, di tipo federale - tuttavia manca ancora una storia o un'opera che offra una descrizione chiara del federalismo libertario. Ciò è probabilmente dovuto al fatto che la prima definizione che si dà dell'anarchia, vale a dire la rappresentazione ideale della società anarchica, è una definizione di tipo negativo: l'alfa privativo $a$ posto dinnanzi al termine archos (traducibile come governo, dominio, potere, autorità), implica in primo luogo una negazione del principio dell'autorità, negazione che accomuna in generale chiunque si riconosca come anarchico. Secondo Giampietro Berti, tale negazione è talmente fondante e comune agli anarchici che «Ciò che si aggiunge in positivo [...] non potrà essere unanime». Da questo carattere intrinsecamente negativo deriverebbe, in ultima analisi, quello utopico dell'anarchia che dunque, in quanto forma di governo, essendo fondata su una negazione, costituisce un problema «irresolubile $»^{20}$. Ne segue che la tematica principalmente posta in rilievo, ogni qualvolta si parli o si studi l'anarchia e il pensiero anarchico, sia per lo più rivolta ai concetti e agli argomenti adottati per contestare le diverse forme di autorità e di potere, in particolar modo la critica allo Stato e alla proprietà. Minore è invece l'interesse che suscitano le finalità e le proposte politiche avanzate storicamente dal movimento anarchico. Se così abbiamo, da un lato, una nutrita letteratura di storie "complessive" sull'anarchia e sull'anarchismo che si risolvono soprattutto in una storia dei protagonisti e del pensiero dei principali teorici, così abbiamo, dall'altro, radi riferimenti in merito alle proposte concrete e praticabili avanzate dagli anarchici e dal movimento anarchico. Eppure già George Woodcock aveva messo in guardia rispetto a una perimetrazione dell'anarchia che guardasse solo ed esclusivamente al suo carattere negativo, descrivendola storicamente come «una dottrina che critica la società esistente, preconizza un nuovo ordine sociale, indica i mezzi per passare dall'uno all'altro» ${ }^{21}$. Proposta dunque, non solo protesta.

\footnotetext{
${ }^{20} \mathrm{G}$. Berti, Il pensiero anarchico cit., p. 12.
} 
Ora, al di là della complessità del definire in maniera compiuta il principio di libertà che viene opposto dagli anarchici a quello di autorità, e al di là della più generale indefinibilità del concetto stesso di libertà, è certo che l'anarchismo abbia particolarmente teso nel corso della sua storia «alla sostituzione dello stato autoritario con qualche forma di libera cooperazione tra individui liberi» ${ }^{22}$. Su una possibile prassi modellata su principi anarchici si è espresso anche Salvo Vaccaro, che ha definito l'anarchismo proprio come «una matrice di prassi politica che, opportunamente elaborata, affinata e calibrata localmente, può offrire uno stile di organizzazione delle comunità informate ai principi di libertà $\rangle^{23}$. Colin Ward ha per di più sostenuto che l'anarchia non sia tanto la rappresentazione di una società futura, quanto «la descrizione di un modo umano di organizzarsi radicato nell'esperienza della vita quotidiana $\rangle^{24}$, dunque non tanto la creazione di un nuovo ordine quanto l'affermazione di pratiche già esistenti ${ }^{25}$. Più precisamente, secondo Woodcock, il mutamento a cui mirano gli anarchici può essere così sintetizzato: «Dissoluzione dell'autorità e del governo, decentramento delle responsabilità, sostituzione degli stati e di analoghe organizzazioni monolitiche con 'federazioni' nelle quali la sovranità torni alle unità prima della società $[\ldots]\rangle^{26}$. Un'idea in tutto concorde è stata espressa da Noam Chomsky, secondo il quale la rappresentazione anarchica consisterebbe in «un sistema di consigli operai, consigli di consumatori, assemblee comunitarie, federazioni regionali, e così via ${ }^{27}$. Ward a sua volta ha posto in rilievo come l'organizzazione sociale affermata dai maggiori pensatori anarchici sia composta da un insieme di gruppi locali di carattere territoriale e produttivo che

si aggregherebbero [...] come le maglie di una rete, una rete di gruppi autonomi. Numerose categorie concettuali concorrono alla definizione della teoria sociale anarchica, tra le altre quelle di azione diretta, di autonomia, di autogestione, di decentramento e di federalismo ${ }^{28}$.

${ }^{21}$ G. Woodcock, L'Anarchia. Storia delle idee e dei movimenti libertari, Feltrinelli, Milano, 1966, p. 5.

${ }^{22}$ Ivi, p. 9. Considerazioni similari furono espresse anche da S. Rota Ghibaudi, cfr. Discussione, in Anarchici e anarchia nel mondo contemporaneo. Atti del Convegno promosso dalla Fondazione Luigi Einaudi (Torino, 5, 6, e 7 dicembre 1969), Fondazione Luigi Einaudi, Torino, 1970, p. 49.

${ }^{23}$ V. A. Scrima, S. Vaccaro, Conversazione su anarchia e autogoverno, La Fiaccola, Ragusa, 1994, p. 11.

${ }^{24} \mathrm{C}$. Ward, Anarchia come organizzazione. La pratica della libertà, Elèuthera, Milano, 2006, p. 12.

${ }^{25}$ In sostanziale accordo con questa idea vi sono anche le considerazioni in V. A. Scrima, S. Vaccaro, Conversazione su anarchia cit., pp. 13-14.

${ }^{26} \mathrm{G}$. Woodcock, L'Anarchia cit., p. 22. Cfr. anche l'intervento di Federica Montseny in Discussione, in Anarchici e anarchia cit., p. 51.

${ }^{27}$ N. Chomsky, Il governo del futuro, Tropea, Milano, 2009, p. 30. 
Il termine federalismo, come si vede, compare sovente per descrivere il nuovo tipo di ordine sociale cui aspirano gli anarchici, ma ancora non ne emerge un profilo limpido che aiuti a capire anche solo approssimativamente quali siano le sue caratteristiche. Tanto più che il termine federalismo non è affatto sufficiente da sé, specie se inteso generalmente come unione di stati dalle ampie autonomie con un governo unico cui sono demandati solo alcuni compiti o poteri. Nonostante la separazione delle attribuzioni possa anche avvenire in modo ascendente, dal basso verso l'alto, «la logica statuale che sorregge questo modello di federalismo riordina i poteri esercitati dal basso lungo una catena saldamente connessa secondo gerarchia di autorità $\rangle^{29}$. Scrima e Vaccaro hanno proposto pertanto il termine «acentramento» intendendo con questo «una posizione di autogoverno liberato in cui l'autonomia radicalmente coerente non è subordinata ad istanze di disciplinamento e di regolazione statuale, bensì è indipendente nelle relazioni di reciprocità $e$ orizzontalità tra comunità e tra singolarità ${ }^{30}$. Il concetto è senza dubbio importante e funzionale a contrapporsi a quello di accentramento o, più in generale, di centro politico, ma ci appare solo in parte soddisfacente: se da un lato riconosce per un federalismo di tipo libertario la necessità di superare lo Stato quale ambito istituzionale di riferimento, dall'altro si limita - nel prosieguo dell'argomentazione a una critica delle convenzionalità delle frontiere interne ed esterne (tra quartieri, amministrazioni locali, comunità nazionali, etc.), senza offrire un'illustrazione compiuta di un paradigma federalista libertario e della sua storia.

Un tentativo descrittivo più compiuto in questo senso è stato tentato da Luigi Di Lembo all'inizio del suo saggio sulla storia del federalismo libertario in Italia ${ }^{31}$. Egli ha avuto inoltre il merito di problematizzare la differenza esistente tra idea libertaria e idea anarchica. Le due rappresentazioni, pur partendo dai cardini comuni dell'individuo e dell'autogestione, sarebbero infatti prossime ma non necessariamente coincidenti. Il federalismo, inteso in senso libertario, privilegerebbe l'autogestione e dunque «non esclude al suo interno forze decisionali di tipo democratico (maggioranza/minoranza) o di ampia delega»; pertanto «il federalismo libertario non esclude forme di organizzazione statale ridotta alle funzioni essenziali e configura una costruzione dalla periferia al centro $\rangle^{32}$. Il federalismo libertario sarebbe dunque più prossimo allo "Stato minimo" che non a una visione a-statale o

${ }^{28}$ C. Ward, Anarchia come organizzazione cit., p. 22. Scrima e Vaccaro si sono particolarmente soffermati sull'istanza di autonomia quale principio fondamentale del pensiero e della prassi anarchiche, cfr. V. A. Scrima, S. Vaccaro, Conversazione su anarchia cit., pp. 15-22.

${ }^{29}$ V. A. Scrima, S. Vaccaro, Conversazione su anarchia cit., p. 31.

${ }^{30}$ Ivi, p. 32. I corsivi sono originali.

${ }^{31}$ L. Di Lembo, Il federalismo libertario cit., ma vedi anche Id., La tradizione dell'anarchismo federato, in L'Unione Anarchica Italiana. Tra rivoluzione europea $e$ reazione fascista (1919-1926), Zero in condotta, Milano, 2006, pp. 13-27. I saggi di Di Lembo ci risultano essere ad oggi gli unici studi che abbiano cercato di tratteggiare una storia - per quanto geograficamente limitata alla penisola italiana - dell'idea federalista libertaria.

${ }^{32}$ Ivi, p. 3. 
anti-statale, viceversa il federalismo anarchico si determina come costruzione orizzontale di solidarietà volontarie e quindi rifiuta

non solo il concetto di governo dall'alto ma anche quello della democrazia e della delega $[. .$.$] non riconosce maggioranze o minoranze ma solo l'oggettivo prevalere di$ una soluzione "tecnica" su altre e il diritto per chi non condivide quella prevalente di provare la propria ${ }^{33}$.

La distinzione posta da Di Lembo è tutt'altro che secondaria, specialmente se si considera il fatto che i termini libertario e anarchico sono spesso adottati come sinonimi. Senza dubbio si tratta di idee prossime, tangenti, capaci di convergere sui valori fondamentali della libertà e dell'individuo, tuttavia se la posizione anarchica è una posizione senza dubbio libertaria, non così il contrario. Il libertarismo può presentare diverse sfumature e letture, tra cui alcune di tipo estremamente liberista e liberale, che sono particolarmente diffuse nel mondo anglosassone ${ }^{34}$. Nel contesto europeo continentale invece il libertarismo corrisponde - di norma - all'accezione socialista del termine, ma può non essere necessariamente un paradigma anarchico, anche se prossimo a tale rappresentazione ${ }^{35}$. A tal proposito non sarà inoltre fuori luogo rammentare come, nel tentativo di ricostruire le origini dell'anarchismo, alcuni autori si siano spinti con la loro ricerca fino alle più antiche manifestazioni di fermenti e concezioni libertarie, costituendo così un coacervo vasto e disomogeneo di pensatori e movimenti che spesso e volentieri poco o nulla hanno a che vedere con l'anarchismo propriamente detto ${ }^{36}$. Se tale albero genealogico può avere una funzione, la ha solo in quanto riconosce il manifestarsi nella storia di tendenze libertarie che contestano l'autorità, la gerarchizzazione e l'accentramento del potere.

Di Lembo coglie dunque un importante discrimine, una differenza specifica fondamentale tra idee libertarie, idee anarchiche e relative rappresentazioni federaliste. Queste ultime, in ambedue i casi, appaiono come paradigmi funzionali alla disgregazione del centralismo e alla valorizzazione delle autonomie, ma con fini che possono differire: per un anarchico il fine ultimo è la distruzione e la dissoluzione assoluta del potere, non così per un libertario che può semplicemente mirare a ridurre al minimo le competenze dello Stato e del potere centrale. In altre

\footnotetext{
${ }^{33} \mathrm{Ibid}$.

${ }^{34}$ Si veda P. Vallentyne, Libertarianism, in The Stanford Encyclopedia of Philosophy, Edward N. Zalta, Spring 2012 Edition

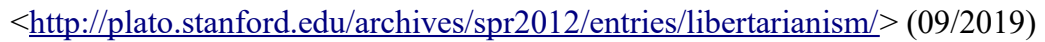

${ }^{35} \mathrm{Si}$ pensi, per fare un esempio, al socialismo gildista sorto in Inghilterra all'inizio del XX secolo intorno alla figura di George Douglas Howard Cole e ispirato a principi federalisti e cooperativisti. Cfr. C. Wyatt, A recipe for a cookshop of the future: G.D.H. Cole and the conundrum of sovereignty, «Capital \& Class», XXX, Autumn 2006, pp. 93-123.

${ }^{36}$ Cfr. soprattutto P. Marshall, Demanding the Impossible. A History of Anarchism, Harper Perennial, London, New York, Toronto and Sydney, 2008, pp. 51-188, ma anche M. Nettlau, Breve storia dell'anarchismo, Edizioni L'Antistato, Cesena, 1964, pp. 1-14, e G. Woodcock, L'Anarchia cit., pp. 31-50.
} 
parole, un libertario potrebbe non voler spingere il processo di indebolimento dello Stato fino alla sua scomparsa e alla conseguente realizzazione dell'anarchia, laddove un anarchico fa proprie le aspirazioni e gli auspici libertari portandoli fino alle loro estreme conseguenze. Ne deriverebbe di conseguenza un'ulteriore complessità, ossia di non poter parlare di un unico modello di federalismo libertario, ma piuttosto di "federalismi libertari": ciò rileverebbe meglio la pluralità di paradigmi federali cui l'aggettivo libertario può condurre. Tuttavia non intendiamo lasciarci intrappolare in questo labirinto terminologico e, pur prendendone atto, riteniamo sia necessario andarne al di là. In questo senso, riteniamo che ad oggi sia incontestabile il fatto che, in generale, il significato estensivo del termine e dell'aggettivo libertario sia appunto quello di anarchico e che, più specificatamente, socialismo libertario sia un termine tradizionalmente utilizzato nell'ambito della storia del pensiero politico per riferirsi appunto all'anarchismo. Quest'ultimo contempla allo stesso tempo nella sua prospettiva tanto la valorizzazione e la difesa della libertà individuale quanto - e qui vi è la sostanziale differenza con altre possibili rappresentazioni libertarie - quella dell'uguaglianza sociale, cercando di trovare una sintesi per la quale

si realizza veramente la libertà individuale solo attraverso il completo dispiegamento dell'uguaglianza sociale e si realizza veramente l'uguaglianza sociale solo attraverso il completo dispiegamento della libertà individuale ${ }^{37}$.

Il federalismo libertario potrà dunque essere un paradigma declinabile secondo diverse prospettive politiche, ma resta principalmente un'elaborazione del pensiero anarchico.

Lo studio delle idee federaliste di Berneri può quindi portare un contributo a questo importante snodo teorico, oltre a colmare una lacuna nella Storia del pensiero politico, poiché gli studi sul federalismo tendono generalmente a non considerare o a ignorare del tutto gli apporti e i contributi degli anarchici nello sviluppo di questa idea $^{38}$. La vicenda berneriana dimostra invece che non solo il movimento anarchico ha dato storicamente una propria originale interpretazione del federalismo, ma anche che ha saputo riflettervi e svilupparla in maniera operativa, aprendosi al dibattito con movimenti e realtà diverse con cui condivideva la fede in questo tipo di organizzazione politica. In altre parole, gli anarchici hanno dato un contributo non trascurabile al pensiero federalista e Berneri, con la sua profonda e continua riflessione, ha dato con ogni probabilità l'ultimo e più alto contributo in questo senso

${ }^{37} \mathrm{G}$. Berti, Il pensiero anarchico cit., p. 29.

${ }^{38}$ Con la sola eccezione delle idee di Pierre-Joseph Proudhon, non vi è in genere traccia di altre idee libertarie negli studi sul federalismo. Un'eccezione notevole è quella di C. Malandrino, Federalismo. Storia, idee, modelli, Carocci, Roma, 1998, che dedica spazio a Bakunin e alla Comune di Parigi (pp. 56-57), alla presenza dei loro indirizzi federali nella Prima Internazionale (pp. 81-83), e al dibattito tra Rosselli e Berneri (pp. 109-110); di quest'ultimo dibattito era già stato ampiamente reso conto in Id., Socialismo e libertà. Autonomie, federalismo, Europa da Rosselli a Silone, Franco Angeli, Milano, 1990, pp. 129135. 
nel corso della prima metà del Novecento. Lo studio del percorso che lo porta fino alla redazione della sua "costituente libertaria", che prende forma e corpo anche nel confronto con altri grandi intellettuali italiani e stranieri del secolo scorso, può portare nuova luce e aprire una nuova finestra nell'ampia stanza della Storia delle dottrine politiche su un aspetto non troppo affrontato o comunque sin qui scarsamente considerato. Aprire una finestra significa non solo poter offrire una prospettiva nuova, ma anche areare il locale facendo nel contempo luce su letture e documenti che possono e devono portare a ripensare, o comunque a riconsiderare, parti che rischiano di restare nella penombra della Storia. È nostra opinione che il progetto di Berneri, che è ad oggi l'unico tentativo documentato di trascrizione di un progetto di costituzione federalista e libertaria attraverso cui tradurre in atto almeno una parte delle idee e dei principi anarchici, non debba correre questo rischio. 


\section{Capitolo 1 \\ Il sentimento e l'impegno: la gioventù e la formazione politica di Camillo Berneri}

\section{Come un giovane socialista divenne anarchico}

Camillo Berneri ha quindici anni quando, nel 1912, si iscrive alla Federazione Giovanile Socialista di Reggio Emilia. È giovanissimo e la sua adesione al socialismo ha i caratteri e la forma di un imperativo morale, di una professione di fede in cui si mescolano moti sentimentali verso la causa degli ultimi unitamente a mitologie di una società giusta ed eguale, a speranze nel progresso sociale e nell'emancipazione delle masse diseredate. Ciò è confermato dalla sua stessa formazione che, nelle memorie della madre Adalgisa Fochi, appare segnata da queste caratteristiche e ammantata dalla tradizione democratica e repubblicana di età risorgimentale di famiglia: lo stesso nome che sceglie per suo figlio - Luigi Camillo - risulta composto dai nomi del bisnonno Luigi, mazziniano e carbonaro, e del nonno Camillo, volontario garibaldino nel 1860 e nel 1866. Nei suoi ricordi Adalgisa Fochi si descrive «imbevuta di garibaldinismo» ed educata ne «il meglio, il romantico, l'umanitario ed anche il cavalleresco dell'epoca dal '21 al '60»; così il piccolo Camillo cresce a sua volta nel mito dell'epopea popolare del Risorgimento, sviluppando un «sentimento dell'ingiustizia delle sorti umane» e uno «spirito della giustizia e del rispetto alla dignità umana ${ }^{39}$.

Il giovane Berneri non può allora che rivolgere le proprie aspettative e il proprio interesse politico al socialismo, erede fin dalla sua fondazione «di tutto quanto s'era

${ }^{39}$ A. Fochi, Con te, figlio mio!, Officina Grafica Franchising, Parma, 1948, p. 14, p. 90 e pp. 92-95. Sulle idee di Adalgisa Fochi e sulla tradizione politica della famiglia si è particolarmente soffermato C. De Maria, Una famiglia anarchica. La vita dei Berneri tra affetti, impegno ed esilio nell'Europa del Novecento, Viella, Roma, 2019, pp. 19-33. Sulla propria infanzia, per parte sua, Berneri dichiara di non avere che pochi ricordi per lo più confusi e frammentari, vedi C. Berneri, Ricordi, in Id., Pensieri e battaglie, Comitato Camillo Berneri, Parigi, 1938, pp. 25-26. 
detto, s'era agitato, s'era operato e s'era creduto nei trascorsi decenni in campo democratico» e la cui propaganda tra le classi popolari si era servita spesso e volentieri di «vaghi motivi ideali, magari miti e legami sentimentali» ${ }^{40}$ provenienti dalla tradizione politica mazziniana o dalla fervente mitologia garibaldina. La comunanza e la continuità tra il movimento democratico-repubblicano e quello socialista sono in altre parole segnate da quella che Giorgio Sacchetti ha felicemente definito come una «koiné dei rivoluzionari» ${ }^{41}$, ossia una serie di aspirazioni e di idee di profondi e radicali mutamenti politici e sociali condivise da entrambe le culture politiche. Ł̀ anche all'insegna di questo linguaggio comune che deve avvenire la formazione e la prima educazione politica di Berneri.

Il Partito Socialista Italiano (PSI) di inizio Novecento, pur avendo raggiunto una notevole maturità politica, ha un'organizzazione poco articolata ed è ancora privo di una programma forte e coerente, con obiettivi precisi. Le sue sezioni si trovano ormai in ogni regione d'Italia, ma non sono sempre in grado di mediare organicamente tra partito e base, tant'è vero che «la propaganda è concepita e praticata soprattutto nelle forme di predicazione della buona novella ${ }^{42}$, proseguendo in parte quella sorta di "religiosità laica" tipica dei repubblicani e dei pionieri del socialismo tramite cui era possibile raggiungere le grandi masse sia urbane che contadine $^{43}$. Tra le personalità in questo senso maggiormente suggestive vi è senza dubbio quella di Camillo Prampolini, dirigente della sezione di Reggio Emilia e deputato parlamentare del PSI: una figura "apostolica", nota soprattutto per la sua propaganda e il suo riformismo profondamente umanitari, popolari e, a tratti, evangelici ${ }^{44}$. È proprio durante una conferenza tenuta da Prampolini a Reggio Emilia

${ }^{40} \mathrm{G}$. Arfé, Storia del socialismo italiano (1892-1926), Torino, Einaudi, Torino, 1965, p. 16. Cfr. anche sul medesimo tema L. Romaniello (a cura di), Le radici del socialismo italiano. Atti del Convegno, Milano, 15-16-17 novembre 1994, Edizioni Comune di Milano Amici Museo del Risorgimento, Milano, 1997, e M. Degl'Innocenti, La patria divisa. Socialismo, nazione e guerra mondiale, Franco Angeli, Milano, 2015, pp. 159-163.

${ }^{41} \mathrm{G}$. Sacchetti, Le culture politiche del giovane Berneri. Un intellettuale fra Arezzo, Firenze e Cortona, in G. Berti, G. Sacchetti (a cura di), Un libertario in Europa. Camillo Berneri: fra totalitarismi e democrazia. Atti del convegno di studi storici, Arezzo, 5 maggio 2007, Edizioni Biblioteca Panizzi, Archivio Famiglia Berneri-Aurelio Chessa in collaborazione con la Provincia di Arezzo, Reggio Emilia, 2010, p. 34.

${ }^{42} \mathrm{G}$. Arfé, Storia del socialismo italiano cit., p. 158. Si vedano in generale le pp. 157-161 per quanto riguarda queste continuità nell'organizzazione e nelle caratteristiche del partito dall'atto di nascita (1892) al Congresso di Reggio Emilia (1912).

${ }^{43}$ Ivi, pp. 19-20. Sulla propaganda del PSI e sulla dinamica tra "alto" e "basso" si veda anche D. Tabor, L'arte della propaganda. Il modello di proselitismo del Psi tra fine Ottocento e inizio Novecento, "Contemporanea», XIV, $\mathrm{n}^{\circ}$ 4, 2011, pp. 579-616. Cfr. inoltre M. Degl'Innocenti, Geografia e istituzioni del socialismo italiano, 1892-1914, Guida, Napoli, 1983, pp. 31-65.

${ }^{44}$ Per un profilo completo sulla figura e sull'attività di Prampolini si vedano il voluminoso lavoro di S. Bianciardi, Camillo Prampolini, costruttore di socialismo, Il Mulino, Bologna, 2012. Si vedano inoltre M. Degl'Innocenti, Camillo Prampolini e il socialismo del suo tempo, 
che il giovane Berneri ricorderà di aver sentito «la prima commozione profonda che il dolore dell'umanità doveva ispirarmi»» ${ }^{45}$.

$\mathrm{Vi}$ è dunque, come già è stato evidenziato ${ }^{46}$, un'innegabile sintonia tra le caratteristiche dell'azione e della predicazione prampoliniane e lo slancio sentimentale e morale dell'impegno socialista del giovane Berneri. Sarà egli stesso a descrivere questo periodo come il proprio «catecumenato socialista» e a riconoscere al proprio maestro «la stoffa di santo e di poeta» insieme a una sincera passione per la causa degli ultimi che ne faceva «uno di quegli uomini che hanno una fede che non insterilisce ${ }^{47}$; caratteristiche, queste, che probabilmente avranno ricordato al giovane Berneri l'altro "apostolo" per eccellenza, Giuseppe Mazzini, nel cui mito e nella cui figura è avvenuta - come si è visto - la sua prima educazione politica.

\section{Cultura e organizzazione, due vie per l'emancipazione}

Fin dalla sua nascita, il movimento giovanile socialista si è dimostrato particolarmente vivace nel proprio attivismo e nell'opera di propaganda e di organizzazione, caratterizzati da una forte vocazione alla causa e da un radicale attaccamento ai principi ideali, cosa che lo ha portato spesso e volentieri a polemiche o a frizioni con la dirigenza del partito, soprattutto se di tendenza riformista. Al momento dell'iscrizione di Camillo Berneri, la Federazione Giovanile Socialista di Reggio Emilia può già vantare una certa autorevolezza, in quanto ha costituito il primo nucleo embrionale della Federazione Italiana Giovanile Socialista (FIGS) aderente al PSI, sorta nel 1907 dopo la scissione con la corrente sindacalista rivoluzionaria ${ }^{48}$.

«Storia e Futuro», VIII, n¹9, febbraio 2009, e G. Boccolari e L. Casali (a cura di), Prampolini e il socialismo reggiano, «L'Almanacco», XX, n³7, dicembre 2001.

${ }^{45}$ C. Berneri, Camillo Prampolini, in Id., Pensieri e battaglie cit., p. 40.

${ }^{46} \mathrm{Su}$ questa prima fase di formazione politica e sull'ascendente esercitato da Camillo Prampolini sul giovane Berneri si sono particolarmente soffermati C. De Maria, Una famiglia anarchica cit., pp. 27-43, C. Strambi, L'inquieta attitudine: Camillo Berneri e la vicenda politica dell'anarchismo in Italia. Primo Libretto, Edizioni Kronstadt, Pisa, 2015, pp. 57-70, P. Adamo, Dai feudi di Camillo Prampolini: Camillo Berneri e la tradizione socialista, in Prampolini e il socialismo reggiano cit., pp. 75-93 e P. C. Masini, Camillo Berneri alla scuola di Prampolini, appendice a C. Berneri, Mussolini, psicologia di un dittatore, Edizioni Azione Comune, Milano, 1966, pp. 105-117. Cfr. anche F. Montanari, La giovinezza di Berneri, «Ricerche Storiche», XXXI, n83, dicembre 1997, pp. 13-15, G. Furlotti, Le radici e gli ideali educativi dell'infanzia di Camillo Berneri, in Memoria antologica, saggi critici e appunti biografici in ricordo di Camillo Berneri, Edizioni Archivio Famiglia Berneri, Pistoia, 1986, pp. 21-22.

${ }^{47}$ C. Berneri, Camillo Prampolini cit., pp. 41-42.

${ }^{48}$ Sulla storia del primo movimento giovanile socialista è ancora indispensabile il riferimento a G. Arfé, Il movimento giovanile socialista. Appunti sul primo periodo (1903-1912), Edizioni Del Gallo, Milano, 1973, in particolare le pp. 25-52 per quel che riguarda lo scontro con la corrente sindacalista rivoluzionaria e la sua successiva scissione. 
La sezione reggiana della FIGS è attiva in varie manifestazioni ricreative e di divulgazione culturale: organizza conferenze e manifestazioni, gite e visite guidate, si occupa della distribuzione della stampa; lo stesso Berneri ricorda i tempi «nelle pianure emiliane, quando divoravo chilometri per giungere a un circolo vinicolo e spiegare il socialismo davanti al busto in gesso del Marx locale ${ }^{49}$. Il contributo di Camillo Berneri a questa battaglia culturale avviene, come per molti dei giovani militanti, anche sulle colonne del periodico «L'Avanguardia», organo della FIGS: qui egli pubblicherà, a partire dal 1914, alcuni contributi culturali e didascalici, volti spesso a divulgare e a rendere accessibili ai giovani lettori figure notevoli di intellettuali, artisti e letterati. I primi articoli e le prime parole pubblicate dal debuttante Berneri non risultano segnalate in nessuna delle rassegne bibliografiche dei suoi scritti ${ }^{50}$, segno che finora nessuno studioso o ricercatore abbia mai operato uno spoglio completo e rigoroso delle annate de «L'Avanguardia» nel periodo in cui il lodigiano ha offerto i suoi primi contributi. Scorrendo i numeri che vanno dal 1912 al 1915 ci siamo imbattuti in un'ampia serie di scritti che contribuiscono invece a rendere in maniera assai efficace il tenore dell'impegno di diffusione culturale del giovane Berneri, trovando articoli che spaziano dal pensiero sociale dello scienziato Alfred Russel Wallace fino alle idee pacifiste nell'arte e alla pittura sociale di Karel Myelback, passando per la vita e il pensiero di grandi figure storiche del socialismo quali Auguste Blanqui, Saverio Friscia e Paule Mink.

Tutto ciò concorre a spiegare come mai proprio Berneri sia stato tra i promotori principali della qualificazione culturale del periodico «L'Avanguardia» e il motivo per cui proprio lui nella Federazione Provinciale Reggiana sarà chiamato a relazionare sul tema della rivista in preparazione al Congresso nazionale della gioventù socialista prevista per il settembre del $1914^{51}$. Il suo impegno si spinge però anche ben al di fuori della stampa, dove possiamo trovarlo impegnato nella fondazione di un circolo di cultura all'interno della sua provincia:

Sabato, 12 - Berneri e Simonini parlano e costituiscono il C[ircolo di] C[ultura] S[ocialista] a Roncocesi ${ }^{52}$.

${ }^{49}$ C. Berneri, Avanti i giovani, in Id., Pensieri e battaglie cit., p. 142.

${ }^{50} \mathrm{Ad}$ oggi il più ampio e completo profilo bibliografico degli scritti di Berneri resta quello di F. Madrid Santos, Camillo Berneri, un anarchico italiano (1897-1937). Rivoluzione e controrivoluzione in Europa (1917-1937), Edizioni Archivio Famiglia Berneri, Pistoia, 1985, pp. 401-534. Da ricordare, seppur non altrettanto ricco, è anche quello di S. D'Errico, Anarchismo e politica. Nel problemismo e nella critica all'anarchismo del ventesimo secolo, il "programma minimo" dei libertari del terzo millennio. Rilettura antologica e biografica di Camillo Berneri, Mimesis, Milano, 2007, pp. 713-734.

${ }^{51}$ Dalla giovine Italia socialista - Dall'Italia settentrionale, «L'Avanguardia», VIII, 19 luglio 1914.

${ }^{52}$ Dalla giovine Italia socialista - Federaz. Provinciale Reggiana, «L'Avanguardia», VIII, 27 settembre 1914. 
Non è dunque un caso trovare il giovane Berneri tra i sostenitori della corrente cosiddetta «culturista», propugnata da Angelo Tasca e fatta propria anche da Prampolini; lo stesso Tasca peraltro ricorderà così il primo incontro col lodigiano:

Lo conobbi allora, nel settembre 1912, sostando a Reggio Emilia prima di recarmi a Bologna, dove si teneva il quarto congresso della Federazione Giovanile Socialista. Berneri apparteneva alla corrente dei cosiddetti "culturisti", che si affermò a Bologna su un mio ordine del giorno [...]. Certamente la sua adesione al nostro "culturismo" non era stata un puro caso, ed egli ne conservò le preoccupazioni e, perché no?, le ingenuità ${ }^{53}$.

I culturisti intendono farsi carico dell'emancipazione e dell'elevazione culturale di contadini e operai e affermano la funzione educativa del partito, agitano la questione della riforma scolastica, si battono per l'istruzione elementare, lottano contro l'analfabetismo, promuovono la nascita di biblioteche e di luoghi dove poter diffondere la cultura a tutto il popolo. Berneri è in perfetta sintonia con questa tendenza, complice ancora una volta la sua formazione che passa attraverso l'esempio della madre, insegnante, e dei suoi racconti su «la vita del grande apostolo dell'istruzione popolare, il Pestalozzi che amò il popolo e che meritò la scritta sul suo monumento "Nulla per sé, tutto per gli altri" $\rangle^{54}$.

Il culturismo genererà un vivace dibattito nella Federazione Giovanile Socialista del tempo, in particolare per via dell'opposizione da parte del giovane Bordiga, che ritiene che la semplice divulgazione culturale non sia in alcun modo di aiuto al movimento operaio e che i proletari farebbero meglio a curarsi di approfondire semmai la propria coscienza di classe ${ }^{55}$. La corrente del culturismo cresce ad ogni modo in un contesto favorevole come quello del riformismo dei socialisti di Reggio Emilia che, specialmente dopo l'amministrazione della città dal 1899 al 1905, è diventato un punto di riferimento per tutte le amministrazioni comunali socialiste. Il socialismo reggiano è un vero e proprio laboratorio sperimentale di un certo successo nello stimolo e nel sostegno fornito alla nascita di cooperative e di associazioni di produttori e di consumatori, di credito e di previdenza sociale, nonché negli investimenti in opere pubbliche: la battaglia culturista avviene dunque su un terreno fertile grazie alle iniziative di riforma che aveva preso a suo tempo

${ }^{53}$ A. Tasca, Camillo Berneri, «Il nuovo Avanti», IV, 22 maggio 1937. Il rapporto tra i due è stato delineato da M. Gervasoni, Il filo rosso della "inappartenenza": Berneri e Tasca, «Rivista Storica dell'Anarchismo», IV, n²1, gennaio-giugno 1997, pp. 85-94.

${ }^{54}$ A. Fochi, Con te, figlio mio cit., p. 68.

${ }^{55} \mathrm{Al}$ dibattito tra Tasca e Bordiga dedica ampio spazio G. Arfé, Il movimento giovanile socialista cit., pp. 108-116, ma cfr. anche P. Spriano, Torino operaia nella Grande Guerra, Einaudi, Torino, 1960, pp. 35-39 e G. Gozzini, Alle origini del comunismo italiano. Storia della federazione giovanile socialista (1907-1921), Dedalo Libri, Bari, 1979, pp. 31-34. Più in generale sul culturismo, si veda la voce «Tasca, Angelo» in F. Andreucci e T. Detti (a cura di), Il movimento operaio italiano. Dizionario biografico, 1853-1943, vol. V, Editori Riuniti, Roma, 1978, pp. 14-15, e M. Degl'Innocenti, Camillo Prampolini e il socialismo del suo tempo cit., pp. 20-27. 
l'amministrazione socialista di Reggio Emilia, impegnandosi operativamente per migliorare l'istruzione e la cultura di operai e contadini, e ottenendo anche risultati considerevoli durante il proprio mandato ${ }^{56}$.

In generale, è possibile affermare che Berneri abbia senza dubbio appreso, in questo contesto e nelle sue prime esperienze di militanza, l'importanza della coniugazione tra slancio sentimentale e impegno concreto diretto su obiettivi ben determinati, e nello specifico il valore delle autonomie individuali e locali che, organizzate "dal basso", possono riuscire a combattere e a contrastare lo Stato, organizzando reti di solidarietà e di fratellanza, strappando importanti concessioni e spazi di libertà, coniugando insomma emancipazione sociale e umana del singolo e della comunità. Noi sappiamo che il movimento socialista, al suo atto di nascita, ereditò questa battaglia politica per l'autonomia delle amministrazioni locali proprio dalla tradizione repubblicana e ciò pone un'ulteriore e interessante continuità tra l'eredità ideale della famiglia di Berneri e la sua prima militanza politica: anche se non è ancora iniziata la sua riflessione sul federalismo, è probabile che il giovane socialista inizi a cogliere in questi primi anni come le autonomie di province e comuni rispetto al potere centrale possano essere una strada da imboccare per trasformare dal basso l'ordinamento esistente, per indebolire lo Stato ed edificare una grande federazione socialista ${ }^{57}$. Non è da escludere, tra l'altro, che una delle prime letture e suggestioni sul tema provengano da un articolo pubblicato su «L'Avanguardia» nella rubrica «Coltura e propaganda socialista», firmato da un certo Mauri e intitolato, appunto, Federalismo e socialismo ${ }^{58}$ : è la prima volta che nell'organo ufficiale della FIGS si parla chiaramente di federalismo come fine del socialismo. L'articolo sottolinea con una lunga rassegna storica come l'autonomia fosse un istinto comune «in quelle epoche e in quei popoli che più contribuiscono allo sviluppo della civiltà», citando in particolare i casi della Grecia antica, dei Comuni medievali e della Comune di Parigi di fine XIX secolo. L'autore esprime la necessità che il socialismo si faccia erede di questa battaglia, accompagnando all'emancipazione dei comuni anche l'emancipazione economica dei lavoratori attraverso libere organizzazioni capaci di estendersi, progressivamente, all'intera umanità:

\footnotetext{
${ }^{56} \mathrm{Su}$ questo punto vedi L. Casali, Sovversivi e costruttori. Sul movimento operaio in EmiliaRomagna, in R. Finzi (a cura di) Storia d'Italia, Le regioni dall'Unità ad oggi. L'EmiliaRomagna, Einaudi, Torino, 1997, in particolare le pp. 488-496 per quel che riguarda i risultati delle politiche scolastiche. Si veda anche, più in generale, M. Degl'Innocenti, Geografia e istituzioni del socialismo cit., pp. 102-107 e pp. 138-153.

${ }^{57}$ Vedi su questo U. Chiaramonte, Il dibattito sulle autonomie nella Storia d'Italia (17961996). Unità-federalismo-regionalismo-decentramento, Franco Angeli, Milano, 1998, pp. 429-445.

${ }^{58}$ E. Mauri, Federalismo e socialismo, «L'Avanguardia», IX, 8 agosto 1915.
} 
E anche qui federazione...dal basso in l'alto autonoma su larga scala. Federazione di società fra loro, e su, su fino al comune, da questo alle provincie, e dalle provincie alla nazione, e da questa alla grande famiglia umana ${ }^{59}$.

\section{Un ostinato antimilitarista}

La semplice battaglia culturista non basterà però al giovane Berneri e al suo desiderio di tradurre in pratica fino all'ultimo dei principi e delle aspirazioni socialiste. Nell'estate del 1914 i venti di guerra che sconvolgeranno l'Europa nel primo conflitto mondiale soffiano anche nell'ancora neutrale Italia, dividendo la penisola tra interventisti e anti-interventisti. Tra questi ultimi troviamo il PSI che, primo fra tutti i partiti politici italiani, aveva incluso tra i suoi contenuti caratteristici il richiamo al pacifismo in nome dell'internazionalismo classista. Il movimento giovanile socialista ha in particolare fatto proprio questo contenuto fin dalla propria fondazione in maniera accesa ed entusiastica, propagandando un vivace e radicale antimilitarismo $^{60}$. La dichiarazione della guerra e il deflagrare delle ostilità hanno però spiazzato completamente la dirigenza del partito che, complice il naufragio della II Internazionale, finirà con l'affermare un neutralismo generico e sostanzialmente imbelle, rinunciando di fatto a opporre una decisa resistenza alle continue manifestazioni degli interventisti. Desiderosi di un'opposizione più forte e radicale sono invece i giovani e la massa dei militanti che non hanno aderito alle suggestioni dell'interventismo democratico o all'idea di una guerra che preluda a una prossima palingenesi rivoluzionaria ${ }^{61}$.

Il giovane Berneri non è da meno e non è quindi un caso trovare, durante l'infuriare della Grande Guerra, suoi articoli antimilitaristi e anti-interventisti, specialmente considerando il clima di confusione e di acceso dibattito che si sviluppa in questo periodo anche tra le fila giovanili ${ }^{62}$. Un suo primo articolo sugli

${ }^{59}$ E. Mauri, Federalismo e socialismo cit.

${ }^{60} \mathrm{Su}$ questo aspetto si è particolarmente soffermato G. Gozzini, Alle origini del comunismo italiano cit., pp. 11-37, ma si vedano anche G. Arfé, Storia del socialismo italiano cit., pp. 190-200, Id., Il movimento giovanile socialista cit., pp. 17-23 e A. Quasi, L'antimilitarismo italiano agli inizi del secolo, «Rivista di storia contemporanea», XI, fsc. 1, gennaio 1982, pp. 123-144.

${ }^{61}$ Sull'atteggiamento tenuto dal PSI durante il conflitto si vedano M. Degl'Innocenti, La patria divisa cit., pp. 169-177 e A. Riosa, La «terza via» del «né aderire né sabotare», in M. Isnenghi e D. Ceschin (a cura di), Gli italiani in guerra. Conflitti, identità, memorie dal Risorgimento ai nostri giorni, vol. III, t. 1, La Grande Guerra: dall'Intervento alla "vittoria mutilata», Utet, Torino, 2008, pp. 139-147. Cfr. inoltre gli ancora indispensabili S. Caretti, Il socialismo italiano e la "grande guerra», in S. Caretti. Z. Ciuffoletti e M. Degl'Innocenti (a cura di), Lezioni di storia del Partito Socialista Italiano, 1892-1976, Cooperativa Editrice Universitaria, Firenze, 1977, pp. 105-125, e L. Cortesi (a cura di), Il PSI e la Grande Guerra, «Rivista storica del socialismo», $\mathrm{X}, \mathrm{n}^{\circ} 32,1967$.

${ }^{62} \mathrm{Sul}$ turbolento scambio di opinioni nella FIGS e sulla posizione assunta da Berneri si è particolarmente soffermato G. Carrozza, Camillo Berneri ed il dibattito antimilitarista nella Federazione Giovanile Socialista, «Università di Firenze, Facoltà di Magistero - Annali dell'Istituto di Storia», III, 1982-1984, Leo S. Olschki, Firenze, 1985, pp. 155-167. Cfr. 
effetti del conflitto sulla neutrale Svizzera, dove si trova in vacanza all'aprirsi delle ostilità, appare sul periodico milanese «La Folla» ${ }^{63}$, ma la maggior parte dei suoi interventi verranno pubblicati su «L'Avanguardia», il giornale della FIGS, e saranno in generale caratterizzati dalla divulgazione delle idee pacifiste e antimilitariste nel mondo della cultura ${ }^{64}$. Non mancheranno però discese in campo più dirette con scritti maggiormente ispirati all'attualità politica e al differenziarsi delle posizioni all'interno del movimento giovanile socialista. Interessante a questo proposito il suo articolo Agli anglofili, volto a demistificare le idee interventiste democratiche o rivoluzionarie che rivolgono la propria simpatia verso l'Inghilterra liberale e progressista contro l'autoritarismo e l'assolutismo tedesco ${ }^{65}$. Ne sortirà, sempre su «L'Avanguardia», una breve polemica con Ernesto Cesare Longobardi ${ }^{66}$ che intende invece difendere la posizione degli "anglofili” e rimproverare al giovane redattore il proprio radicalismo anti-interventista. Nel numero successivo Berneri risponderà con una certa umiltà al dirigente socialista, ma precisando allo stesso tempo la propria posizione sottolineando come l'emancipazione sociale e politica non sia «né francese né inglese, ma interamente internazionale come internazionale è stata ed è la lotta per conquistarla» ${ }^{67}$.

Ancora più interessante è però un suo articolo pubblicato il 20 dicembre 1914, fino ad oggi mai citato né censito, in cui il giovane militante traccia un profilo biografico del socialista francese Auguste Blanqui col fine di sottrarlo all'utilizzo che ne sta allora facendo Mussolini, da poco uscito dal PSI per propugnare la sua linea interventista e rivoluzionaria:

«Chi ha del ferro ha del pane». È una massima rivoluzionaria del rivoluzionario Blanqui.

Mussolini l'ha inchiodata sul frontone dell'intervenzionista Popolo d'Italia - Parlare di Blanqui oggi è utile principalmente per due ragioni. La prima perché Blanqui da noi è stato ed è tuttora un semi-sconosciuto [...]. La seconda, e potrebbe essere anche la prima, perché è stridente il contrasto fra Blanqui e Mussolini. Il rivoluzionario intervenzionista, megalomane agitatore della bandiera dello scisma,

anche, più in generale, G. Gozzini, La Federazione Giovanile Socialista tra Bordiga e Mussolini (1912-1914), «Storia contemporanea», XI, $\mathrm{n}^{\circ}$, febbraio 1980, specialmente le pp. 115-125, e Id., Alle origini del comunismo italiano cit., pp. 39-51.

${ }^{63} \mathrm{C}$. Berneri, Effetti di guerra in un paese di pace, «La Folla», III, 16 agosto 1914.

${ }^{64} \mathrm{Il}$ primo articolo a essere pubblicato sarà proprio di questo tenore, vedi C. Berneri, Un pittore e un poeta pacifisti: Rubens e Fagiuoli, «L'Avanguardia», VIII, 1 novembre 1914.

${ }^{65}$ C. Berneri, Agli anglofili, «L'Avanguardia», VIII, 8 novembre 1914. Si veda anche Id., Riabilitazioni guerraiole, «L'Avanguardia», IX, 14 febbraio 1915, che similmente denuncia l'ipocrisia di quanti concentrino le proprie condanne sui soli crimini di guerra e brutalità commessi dai tedeschi.

${ }^{66}$ E. C. Longobardi, Le confessioni di un anglofilo, «L'Avanguardia», VIII, 22 novembre 1914. Sul personaggio vedi «Longobardi, Ernesto Cesare» in F. Andreucci e T. Detti (a cura di), Il movimento operaio italiano. Dizionario biografico, 1853-1943, vol. III, Editori Riuniti, Roma, 1977, pp. 152-154.

${ }^{67}$ C. Berneri, Confessioni di un anglofobo, «L'Avanguardia», VIII, 6 dicembre 1914. 
ha fatto suo un motto, un pensiero di Blanqui; il rivoluzionario che non ha conosciuto le «crisi d'animo» ${ }^{68}$.

Dopo aver ripercorso la vicenda politica e biografica del socialista francese, Berneri rileva che questi «ha combattuto la sua guerra senza chiedere nulla, né onori, né cariche, né denaro. Non ha mai curato i suoi interessi, ha dato la sua vita alla sofferenza del carcere, l'ha esposta nelle vie, sulle piazze, sulle barricate» ${ }^{69}$. Il giovane socialista prende spunto dalla saldezza dell'idea e dell'azione di Blanqui per lanciare un appello ai suoi compagni affinché mantengano la loro fede e il loro impegno altrettanto saldi:

Oggi che abbiamo visto tanti che noi credevamo uomini dalla fede di dinamite, sbriciolare a poco a poco la loro coscienza, oggi che abbiamo visto sparire tanti sogni, cadere tante illusioni, abbiamo però sempre la nostra storia che è storia gloriosa. Gridiamolo alto che abbiamo avuto i rivoluzionari veri che non hanno mai tergiversato, che non si sono mai dichiarati vinti; gridiamolo e copriamo le voci maligne quanto idiote di coloro che godendo di questo nuovo scisma ci predicano la fine del socialismo. Copriamole oggi come ieri abbiamo coperto il De profundis che i guerrafondai cantano per la pretesa morte dell'Internazionale col grido di: abbasso il militarismo! Rivelando così che s'era fallita l'internazionale dei vecchi pieni ancora di pregiudizi, con la mente ingombra ed offuscata da odii di razza, s'era fatta più viva, più forte la nuova Internazionale, quella del sovversivismo della nuova generazione $^{70}$.

Le tensioni tra posizioni interventiste e anti-interventiste tengono alto il clima di tensione e non tarderanno ad agitare anche la pacifica Reggio Emilia: il 25 febbraio 1915 è previsto in città un comizio interventista cui prenderà parte anche Cesare Battisti; i socialisti reggiani intendono opporsi e il deputato Zibordi si mette così alla testa di una dimostrazione neutralista. L'atmosfera incandescente - in cui Berneri rischia addirittura di essere scambiato per uno studente interventista - culmina in uno scontro che si concluderà con un tragico eccidio: la polizia per disperdere la folla spara nel mucchio uccidendo due giovani (Fermo Angioletti e Mario Bosicchi) e facendo una decina di feriti ${ }^{71}$.

Se da un lato l'esperienza conferma nel giovane Berneri il desiderio di partecipare in prima persona alla lotta senza timore delle conseguenze, dall'altro nel commentare i fatti di Reggio Emilia egli fa mostra di allinearsi alla tendenza espressa dalla dirigenza del partito, invitando i suoi a far in parte svampare la

${ }^{68}$ C. Berneri, Augusto Blanqui, «L'Avanguardia», VIII, 20 dicembre 1914.

${ }^{69}$ Ibid..

${ }^{70} \mathrm{Ibid}$.

${ }^{71}$ L'intero episodio e i successivi stati d'animo di Berneri sono narrati in A. Fochi, Con te, figlio mio cit., pp. 118-119, ma si vedano anche le ricostruzioni di P. C. Masini, Camillo Berneri alla scuola di Prampolini cit., pp. 105-107 e F. Montanari, La giovinezza di Berneri cit., pp. 17-18. 
propria febbre rivoluzionaria ${ }^{72}$. Il giovane redattore non mancherà di puntare il dito contro i nazionalisti e la stampa italiana, accusati di aver contribuito ad alimentare un insopportabile clima di tensione, indicando nel governo il maggiore responsabile dell'eccidio e delle violente repressioni che egli definirà in seguito vere e proprie piaghe d'Italia ${ }^{73}$. Resta il fatto però che, all'indomani dell'episodio, Berneri rientri o comunque cerchi di rientrare più ordinatamente nei ranghi del partito e inviti a una moderata prudenza, andando in forte controtendenza rispetto alla sua effervescente indole militante. Certamente deve avere un peso su questo mutamento di prospettiva il trauma dell'evento e l'angoscia per la morte dei due compagni nella

Reggio socialista mite e buona, educata alla scuola di fratellanza e di giustizia di Camillo Prampolini, $[. .$.$] che fra tutte le città rosse d'Italia è nominata come la città$ esemplare per la calma, per la tolleranza e la libertà d'opinione ${ }^{74}$.

A questi aspetti si devono anche aggiungere da un lato la sua giovane età e, di conseguenza, il suo essere facilmente suggestionabile, dall'altro anche le influenze che devono avere su di lui le preoccupazioni espresse dalla madre e l'influenza autorevole e decisiva di Prampolini, apostolo della non violenza e della calma. Il conformarsi alla linea del partito non deve in ogni caso essere stata una scelta fatta di buon grado e con piena convinzione considerando che, di lì a pochi mesi, Berneri si dimetterà dalla FIGS.

\section{Passeggiando sotto i portici della via Emilia: l'adesione all'anarchismo}

Qualcosa si era mosso già in quell'estate del 1914, durante la quale Berneri aveva conosciuto alcuni anarchici reggiani e cominciato a frequentare il circolo anarchico Spartaco. Ha stretto amicizia in maniera particolare con Torquato Gobbi, tipografo e legatore di libri, di cui è anche vicino di casa e di cui subisce il fascino intellettuale: sebbene sia stato costretto dalle modeste condizioni economiche della famiglia a interrompere gli studi al livello elementare, Gobbi è un appassionato autodidatta e in quel momento è senza dubbio «il militante più rappresentativo dei gruppi anarchici reggiani» ${ }^{75}$. L'affinità elettiva tra Gobbi e Berneri, di nove anni più giovane, è forte e contagiosa, tant'è che quest'ultimo avrà modo in seguito di ricordare la crucialità dell'influenza ricevuta dalla «pacata dialettica» di quello che

${ }^{72}$ C. Berneri, Dopo i fatti di Reggio Emilia, «L'Avanguardia», IX, 14 marzo 1915.

${ }^{73}$ Vedi C. Berneri, Piaghe di casa nostra, «L'Avanguardia», IX, 4 aprile 1915, che intende mostrare come il clima repressivo in Italia sia dovuto a una forza pubblica reazionaria e intollerante, non interessata al mantenimento dell'ordine come altrove in Europa dove gli incidenti sarebbero assai più ridotti.

${ }^{74}$ C. Berneri, Dopo i fatti di Reggio Emilia cit.

${ }^{75}$ A. Senta, Note su Torquato Gobbi, un anarchico problematico, «Clio», XLIV, n³, settembre-ottobre 2008, p. 351. Si veda anche «Gobbi, Torquato» in M. Antonioli et al. (diretto da), Dizionario biografico degli anarchici italiani, vol. I, Edizioni Biblioteca Franco Serantini, Pisa, 2003, pp. 739-742 e F. Montanari, Voci del Plata: vita e morte di Torquato Gobbi, Bertani, Verona, 1997. 
egli definisce essere stato il suo «maestro, nelle sere brumose, lungo la via Emilia» ${ }^{76}$; Gobbi ricorderà a sua volta come il suo nuovo amico «soleva distrarsi con interminabili passeggiate lungo i portici di Via Emilia discutendo con me della politica del giorno e facendo insieme delle enormi scorpacciate di sociologia» ${ }^{77}$. La considerazione che Berneri ha dell'amico - tanto da arrivare a definirlo suo maestro - deve essere adeguatamente considerata e conseguentemente deve rendere più complessa e completa l'interpretazione di Claudio Strambi, che ha parlato del «debole per l'autorevolezza» del giovane Berneri solo in relazione a Prampolini e alla madre ${ }^{78}$.

Tra 1914 e 1915 il giovane Berneri ha così avuto modo di confrontare tra loro socialismo e anarchismo su diverse questioni, ha avuto modo di coglierne le affinità ma soprattutto le divergenze, in particolare ha potuto guardare con occhi diversi il riformismo prampoliniano che, sul terreno delle lotte sociali, veniva particolarmente avversato da Gobbi, tanto da fargli affermare che a Reggio Emilia non esistesse la lotta di classe. Sarà però sul tema dell'antimilitarismo e dell'opposizione alla guerra che Berneri troverà la differenza più eclatante tra $\mathrm{i}$ due fronti: dopo il momento di fiamma della cosiddetta Settimana Rossa $(1914)^{79}$ egli, come molti militanti, ha visto sfaldarsi il fronte delle forze politiche contrarie alla guerra, con i repubblicani e frange del sindacalismo rivoluzionario che si sono dichiarati a favore dell'intervento, mentre i socialisti si dichiareranno, come detto, neutralisti assumendo però un atteggiamento oscillante - alcuni elementi arriveranno a simpatizzare per il blocco dell'Intesa, altri opereranno vere e proprie scissioni fondando gruppi interventisti. Gli anarchici da questo punto di vista si presentano più coesi e compatti come movimento e, al netto di «una serie di sporadici e slegati casi personali, qualcuno di rilievo, qualcun altro di nessun rilievo ${ }^{80}$, coerenti al loro antimilitarismo di stampo tanto classista quanto umanitario; tali caratteristiche favoriranno peraltro un

${ }^{76}$ Il ricordo di Gobbi si trova nel saggio C. Berneri, L'operaiolatria, Edizioni Archivio Famiglia Berneri, Pistoia, 1987, p. 5. Apparso inizialmente sotto forma di breve articolo in «Guerra di Classe», 30 aprile 1932, una sua versione più ampia e articolata viene poi pubblicata in forma di opuscolo dal Gruppo d'Edizioni Libertarie, Brest, 1934. Il saggio è riprodotto in Id., Pietrogrado 1917 Barcellona 1937. Scritti scelti, a cura di Pier Carlo Masini e Alberto Sorti, La Fiaccola, Ragusa, 1990, pp. 144-160, in Id., Anarchia e società aperta, a cura di Pietro Adamo, M\&B, Milano, 2001, pp. 142-151, e in Id., Scritti scelti, Zero in condotta, Milano, 2013, pp. 265-276.

${ }^{77}$ Le parole di Gobbi sono citate in A. Fochi, Con te, figlio mio cit., p. 117.

${ }^{78}$ Cfr. C. Strambi, L'inquieta attitudine: Camillo Berneri e la vicenda politica dell'anarchismo in Italia. Primo Libretto cit., pp. 64-65.

${ }^{79} \mathrm{Su}$ questo tema vedi A. Senta (a cura di), La rivoluzione scende in strada: la settimana rossa nella storia d'Italia, 1914-2014. Atti del convegno organizzato dall'Archivio storico della Federazione anarchica italiana, Imola, sabato 27 settembre 2014, Zero in condotta, Milano, 2016, e M. Severini (a cura di), La settimana rossa, Aracne, Roma, 2014. Cfr. inoltre L. Lotti, La settimana rossa, Le Monnier, Firenze, 1972, e M. Degl'Innocenti, La crisi del riformismo e gli intransigenti (1911-1914), in G. Sabbatucci (a cura di), Storia del socialismo italiano, vol. II, L'età giolittiana (1900-1914), Il Poligono, Roma, 1980, pp. 435-440. 
avvicinamento tra giovani militanti socialisti e anarchici nell'azione diretta contro la guerra $^{81}$. Berneri, attivo e radicale nella propria opposizione alla guerra più di quanto non lo sia il suo partito e - ancor di più - la propria sezione di appartenenza, non potrà che guardare con ammirazione l'impegno costante dell'amico Torquato Gobbi contro il militarismo e la sua militanza a favore di Augusto Masetti ${ }^{82}$, che porterà tra l'altro il tipografo anarchico a promuovere a Reggio Emilia un comizio in sua difesa.

L'anno compreso tra l'estate del 1914 e quella del 1915 è dunque un periodo di profonda inquietudine e di tormento per il giovane militante socialista che sempre più difficilmente riesce a vedere nelle iniziative del proprio partito quello spirito e quelle idee che lo avevano spinto a iscriversi alla FIGS. Il binomio tra pensiero e azione, formula di mazziniana memoria che aveva certo influenzato la personalità di Berneri, risulta sempre più drammaticamente divaricato e pone $\mathrm{i}$ primi quesiti al giovane socialista. Egli continua nonostante tutto a svolgere azione di propaganda e di organizzazione ${ }^{83}$, mettendo in gioco anima e corpo e senza lasciarsi tentare dal massimalismo rivoluzionario che egli ritiene vago e confuso dal punto di vista

${ }^{80} \mathrm{P}$. C. Masini, Gli anarchici italiani tra "interventismo" e "disfattismo rivoluzionario", «Rivista storica del socialismo», II, ${ }^{\circ}{ }^{5}$, gennaio-marzo 1959 , p. 208 , sull'interventismo di alcuni anarchici si veda M. Antonioli, Gli anarchici italiani e la prima guerra mondiale. Lettere di anarchici interventisti (1914-15), «Rivista Storica dell'Anarchismo», II, n, gennaio-giugno 1995, pp. 77-107. Cfr. inoltre A. Dadà, Gli anarchici italiani fra guerra di classe e reazione, in I. Barbadoro (coordinata da), Storia della società italiana, vol. XXI, La disgregazione dello Stato liberale, Teti, Milano, 1982, pp. 376-381.

${ }^{81}$ Sui rapporti tra anarchici e movimento giovanile socialista, vedi G. Sacchetti, Compagni di strada. Gli anarchici italiani e la questione delle alleanze (1914-1944), in L'Unione Anarchica Italiana. Tra rivoluzione europea e reazione fascista (1919-1926), Zero in condotta, Milano, 2006, pp. 112-114. Sull'antimilitarismo degli anarchici resta ancora un riferimento indispensabile G. Cerrito, L'antimilitarismo anarchico in Italia nel primo ventennio del secolo, Edizioni RL, Pistoia, 1968, in particolare le pp. 36-49 per quel che riguarda la posizione assunta dal movimento anarchico tra Settimana Rossa e ingresso in guerra dell'Italia, ma vedi anche F. Giulietti, Storia degli anarchici italiani in età giolittiana, Franco Angeli, Milano, 2012, pp. 309-320, pp. 248-263.

${ }^{82}$ Augusto Masetti (1888-1966) è stato un anarchico e antimilitarista italiano che, chiamato alle armi durante la Guerra di Libia (1911) si rese protagonista di un radicale atto di insubordinazione sparando un colpo di fucile al colonnello Stoppa. Per questo gesto verrà arrestato, processato e dichiarato incapace di intendere e di volere, stimolando così la nascita di un'intensa mobilitazione in suo favore da parte di anarchici, repubblicani e socialisti che culminerà nella Settimana Rossa. Su questa vicenda si vedano L. De Marco, Il soldato che disse no alla guerra: storia dell'anarchico Augusto Masetti (1888-1966), Spartaco, Santa Maria Capua Vetere, 2003, ma per un più preciso inquadramento storico e politico cfr. R. Zani, La figura di Augusto Masetti, in A. Senta (a cura di), La rivoluzione scende in strada cit., pp. 173-190 e G. Cerrito, L'antimilitarismo anarchico cit., pp. 22-33.

${ }^{83} \mathrm{La}$ prefettura di Reggio Emilia annota, tra l'altro, un suo tentativo di tenere un conferenza contro la guerra a Genova nel 1915, interrotto dalle violenze degli interventisti, si veda Prefettura di Reggio Emilia, Cenno biografico, 20 luglio 1916, in ACS, Min. In., CPC, Berneri Camillo, b. 537, fsc. I. 
ideologico, caratteristiche che finiranno per condurre alcuni dei suoi elementi alla deriva interventista. Eppure la combattività e la passione politica di Camillo Berneri si trovano a essere sempre più smorzate e costrette nella linea etica, ma sostanzialmente immobile, del riformismo del PSI.

\section{La frattura con la Federazione Giovanile Socialista}

Nel novembre del 1915 Berneri ha maturato la sua decisione e abbraccia infine la causa dell'anarchismo. Tale passaggio è segnato dalla sua famosa lettera di dimissioni dalla FIGS. Parlandone a distanza di tempo con la figlia Maria Luisa, egli ricorda di averla scritta «in un caffè, su della carta che avevo comprato in un Sale e Tabacchi all'uscita da una riunione» e di averla spedita un venerdì sperando che venisse recapitata il giorno successivo, prima della usuale riunione ${ }^{84}$. Giacché è uso leggere le lettere di dimissioni durante le riunioni, Berneri vorrebbe scrivere qualcosa che possa essere utile alla propaganda anarchica, che possa scuotere alcuni ferventi spiriti simili al suo tra i giovani socialisti e spingerli così a loro volta verso la sua stessa scelta. Il giovane anarchico deve essere molto convinto dell'efficacia del suo scritto, tant'è vero che un anno dopo, nel 1916, lo ripubblicherà sul settimanale pisano «L'Avvenire Anarchico»: siamo nel pieno della Grande Guerra, le polemiche entro la FIGS non si sono ancora placate e Berneri ritiene pertanto utile riproporre la propria lettera di dimissioni, probabilmente con la speranza che essa possa rispondere a quei sintomi di «sano risveglio» e a quel manifestarsi di «una nuova e sana crisi di spiriti e di coscienze» tra i giovani socialisti, conquistando così qualche militante all'anarchismo ${ }^{85}$. L'oramai giovane anarchico fa leva sull'entusiasmo e la fede rivoluzionaria che, come era stato per lui, li avevano portati ad aderire alle idee socialiste, ma che si erano trovati poi spiazzati «di fronte all'abisso che separa il pensiero dall'azione» e indica i principali responsabili di questa frustrazione nei dirigenti «pseudorivoluzionari» ${ }^{86}$. Berneri tenta di farsi interprete di questo conflitto generazionale e politico, della crisi spirituale che attraversa gli animi della FIGS, ponendo l'accento sul grande valore che hanno le energie e gli auspici dei giovani militanti per un movimento politico di natura e di intenti sovversivi e indagando come i fermenti e i moti d'animo dei giovani portino questi ultimi a vedere degli estranei - se non addirittura degli avversari - nei dirigenti e nei socialisti adulti:

vedete dei proletari organizzati che non sono, in buona parte, che degli aspiranti borghesi $[. .$.$] , per un egoistico, arido senso di lotta di classe, vedete una pleiade di$ avvocatucoli di provincia aspiranti ai seggi comunali in attesa di quelli parlamentari;

\footnotetext{
${ }^{84}$ C. Berneri, Lettera ai Giovani socialisti, in Id., Pensieri e battaglie cit., p. 33. La raccolta riproduce la lettera solo per estratti.

${ }^{85}$ Id., Lettera aperta ai Giovani socialisti, «L'Avvenire Anarchico», IV, 28 luglio e 4 agosto 1916. La lettera verrà in seguito pubblicata come opuscolo, cfr. C. da Lodi [C. Berneri], Lettera aperta ai giovani socialisti di un giovane anarchico, Tipografia "La Poligrafica", Firenze, 1920.

${ }^{86} \mathrm{Ibid}$.
} 
una schiera di professionisti falliti, di ex-operai poltroni ed ambiziosi, dirigenti le organizzazioni, capitananti le agitazioni finenti poi in ordini del giorno e in accomodamenti padronali o governativi ${ }^{87}$.

La causa del popolo, prosegue Berneri, ha bisogno di speranze, ma soprattutto di vedere che si lotta concretamente per la loro realizzazione; il proletariato non può accontentarsi solo di discorsi, ma ha anche bisogno di eroi e di martiri, mentre il Partito Socialista si sta trovando a ripetere «la traiettoria della potenza morale del Cristianesimo, che divenne potente per i suoi martiri e decadde col cessare del sacrificio dei suoi seguaci» ${ }^{88}$. Il parallelo col cristianesimo non deve essere, riteniamo, casuale, considerando il carattere spesso messianico e religioso - seppure in maniera laica - che il socialismo doveva avere per molti militanti, in particolare per quelli della Reggio Emilia prampoliniana da cui Berneri proveniva. Dal momento però che è proprio la volontà di ribellione dei giovani a essere arginata, sottovalutata, quando non è irrisa e sbeffeggiata, dall'atteggiamento estremamente moderato dei dirigenti riformisti, Berneri rivolge un appello ai suoi oramai excompagni affinché non perdano la loro migliore audacia e il loro entusiasmo tentando di far valere le proprie idee all'interno del partito e di riportarvi un autentico e fresco spirito rivoluzionario, «e se vedrete spezzare i vostri bisturi sanatori sui bubboni del pseudo-rivoluzionarismo vile e disonesto verrete a noi. $\mathrm{Ne}$ sono certo» ${ }^{89}$.

La convinzione e la decisa critica al Partito Socialista emergono chiaramente dalla lettera berneriana del novembre 1915; eppure la sera del giorno dopo, mentre sta passeggiando sotto i portici della via Emilia, viene chiamato dai suoi compagni alla riunione. Convinto che essi non abbiano ricevuto le sue dimissioni, Berneri domanda se non abbiano avuto la sua lettera; gli viene risposto che l'hanno ricevuta, ma viene comunque invitato a prendere parte alla riunione.

Allora andai. Ed ebbi una delle più vive emozioni della mia vita: quella di essere chiamato a presiedere l'ultima riunione alla quale partecipavo. $\mathrm{Fu}$ un gesto di simpatia del quale soltanto più tardi vidi l'enorme valore di educazione politica. Allora vidi in esso la prova che mi volevano bene e il distaccarmi da essi mi gonfiava il cuore di commozione ${ }^{90}$.

In quella stessa occasione avviene anche il suo primo e unico breve contatto diretto avuto con Prampolini che, venuto a sapere delle sue dimissioni, lo manda a chiamare per parlargli. Gli dirà, semplicemente: «"Dunque ci lascia”. Ma soggiunse: "Ma resta sempre nel socialismo"»" . Le parole del vecchio maestro ci aiutano a dare

\footnotetext{
${ }^{87}$ C. Berneri, Lettera aperta ai Giovani socialisti, «L'Avvenire Anarchico», IV, 28 luglio e 4 agosto 1916.

${ }^{88}$ Ibid.

${ }^{89}$ Ibid.

${ }^{90}$ C. Berneri, Lettera ai Giovani socialisti, in Id., Pensieri e battaglie cit., p. 33.

${ }^{91}$ Id., Camillo Prampolini cit., p. 41.
} 
la misura di ciò che è avvenuto nell'animo del giovane Berneri: in lui non si è affatto esaurita quella spinta ideale e sentimentale che lo aveva condotto, tre anni prima, ad aderire al socialismo e all'impegno per realizzare una società di liberi ed eguali attraverso la passione rivoluzionaria e un attivismo concreto ma radicale. Come ha ben sottolineato Masini, la sua conversione all'anarchismo è dettata «da una rivolta di ordine morale più che da una scoperta intellettuale» ${ }^{92}$, ed è lo stesso Berneri a confermarlo quando, negli anni del fuoruscitismo, scrive al giellista Libero Battistelli di aver «abbandonato il movimento socialista perché continuamente mi sentivo dare dell'anarchico ${ }^{93}$; in maniera simile si esprimerà proprio con il vecchio compagno Tasca, sostenendo di essere «diventato anarchico a forza di sentirmi trattare come tale dagli Zibordiani di emiliana memoria. E avevano ragione ${ }^{94}$.

In altre parole, non c'è nulla di nuovo nell'animo del giovane Camillo Berneri se non il desiderio di una più stretta coniugazione tra pensiero e azione, la volontà di non dover continuamente comprimere e tenere a freno la propria passione per sterili e imbelli questioni strategiche di partito. L'anarchismo, agli occhi di Berneri, appare come una corrente politica più pura e coerente nel suo farsi interprete del sogno di emancipazione umana e di classe del socialismo. Berneri non ha insomma abbandonato il movimento socialista, ha solo abbandonato il Partito Socialista, ed è significativo che ad affermarlo sia quello stesso Prampolini che nel 1892, all'atto di fondazione del partito a Genova, aveva invitato gli anarchici contrari all'organizzazione di un partito per la lotta elettorale e legalitaria ad abbandonare l'aula ove si svolgeva il congresso.

Il 14 novembre 1915 la redazione de «L'Avanguardia» annuncia le dimissioni di Berneri parlando di «impazienza» ${ }^{95}$ : è dunque da correggere la ricostruzione del Montanari che ha datato le dimissioni di Berneri al luglio $1916^{96}$. Come si è visto si tratta di una separazione senza astio né veleno: la collaborazione del giovane anarchico con l'organo ufficiale della FIGS proseguirà ancora, seppur sporadicamente, fino all'ottobre 1916.

\section{Un racconto libertario}

Proprio il suo ultimo contributo al periodico dei giovani socialisti, finora mai pubblicato né citato in alcuna rassegna bibliografica di scritti berneriani, ci pare meritevole di attenzione come prima manifestazione di idee ravvicinabili al

\footnotetext{
${ }^{92}$ P. C. Masini, La formazione intellettuale e politica di Camillo Berneri, in Atti del Convegno di studi su Camillo Berneri, Milano, 9 ottobre 1977, La Cooperativa Tipolitografica, Carrara, 1979, p. 11.

${ }^{93}$ Lettera di Camillo Berneri a Libero Battistelli (s.l., s.d., ma probabilmente 1929), in C. Berneri, Epistolario inedito, vol. I, a cura di Aurelio Chessa e Pier Carlo Masini, Edizioni Archivio Famiglia Berneri, Pistoia, 1980, p. 18.

${ }^{94}$ Lettera di Camillo Berneri ad Angelo Tasca (Parigi, 1935), in ivi, p. 39.

${ }^{95}$ Impazienza, «L'Avanguardia», IX, 14 novembre 1915.

${ }^{96}$ F. Montanari, La giovinezza di Berneri cit., p. 19. Il Montanari deve aver probabilmente ritenuto che le dimissioni berneriane coincidessero con la pubblicazione della sua lettera su «L'Avvenire Anarchico».
} 
comunalismo libertario e, in generale, alla potenzialità rivoluzionaria delle autonomie locali contro il potere centrale e i suoi rappresentanti. L'articolo Novella Storica. L'Ercole di Valle d'Illiez ${ }^{97}$ è la riproduzione di un piccolo racconto popolare svizzero di fine XVIII secolo che tratta di Gros Bellet, sorta di eroe dei cantoni della Valle d'Illiez e animatore, con la sua forza sovrumana, della loro rivolta contro le angherie del governatore regionale.

Eravamo all'8 settembre 1790, nella Svizzera del cantone di Valais, alla fiera di Monthey. Fra la gente di montagna riunita per i suoi scambi, v'erano molti paesani della Valle d'Illiez. Costoro erano molto adirati contro il governatore della regione, Ildebrando-Arnaldo Schinner, che, per delle cose da nulla, moltiplicava le vessazioni, pronunciava delle ammende, faceva procedere a dei sequestri e distribuiva la prigione ${ }^{98}$.

Lo scorcio del mercato, luogo principale di riunione e discussione tra individui, è ideale per proporre l'idea del formarsi spontaneo di gruppi che si oppongono all'autorità locale, spinti e aggregati dal malcontento per le sue prepotenze e per i suoi abusi di potere. In questo scenario, racconta Berneri, giungono tre guardie che conducono una giumenta al castello, preceduti dal governatore; si noti come al mercato, simbolo dell'aggregazione di produttori e lavoratori, venga contrapposto il palazzo, luogo di potere e di soprusi ai danni del popolo. Tornando alla narrazione, Berneri ci racconta come poco distante dalle guardie e dal governatore vi sia Gros Bellet, infuriato per l'ingiusto sequestro della bestia, avvenuto col pretesto del mancato pagamento di una multa per un'accusa da cui era peraltro stato assolto dalla Dieta. Nel prosieguo della novella, Gros Bellet irrompe nel palazzo durante il pranzo, reclamando la propria giumenta e utilizzando la propria forza per minacciare il governatore. Riottenuto ciò che gli spetta, egli ammonisce l'autorità locale: «Sig. governatore, il popolo è furibondo contro di voi, pensate alla vostra vita». La vicenda ha richiamato l'attenzione della folla presente al mercato e subito il popolo inizia a organizzarsi spontaneamente contro il governatore:

Si formarono dei capannelli di montanari che presero a commentare il fatto, si aggiunsero ad essi molti curiosi ed alcuni, entusiasmati dall'esempio di energia dato da Gros-Bellet, cominciarono a gridare: "È venuto il giorno di cacciare il governatore! Abbasso i tiranni! Morte a Schinner!” Il vocio divenne sempre più intenso; le grida rivoluzionarie si fecero più frequenti e più entusiaste ed il baccano nelle osterie e nelle strade fu tale che Schinner, rimesso dai suoi terrori, mandò una guardia ad intimare ai contadini di tacere $[\ldots]^{99}$.

Il racconto proposto da Berneri è di fatto una rappresentazione della spontaneità rivoluzionaria e dell'aggregarsi di piccoli gruppi corrispondenti a unità territoriali e

\footnotetext{
${ }^{97}$ C. Berneri, Novella storica. L'Ercole di Valle d'Illiez, «L'Avanguardia», X, 29 ottobre 1916. ${ }^{98}$ Ibid.

${ }^{99}$ Ibid.
} 
sociali in sommossa contro un rappresentante autoritario del potere centrale. La narrazione prosegue:

La guardia, impaurita dall'aspetto minaccioso della folla, ritornò precipitosamente al castello. La fuga della guardia diede ancor più coraggio agli improvvisati agitatori che presero a gridare: “Al castello! All'armi! Cacciamo il governatore!” La folla cominciò ad entusiasmarsi a tali grida, cominciò a tumultare e poco dopo una cinquantina di montanari armati di randelli e di picche corsero all'assalto del castello $^{100}$.

Il sommovimento rivoluzionario è riuscito rapidamente ad auto-organizzarsi e a preparare l'offensiva contro l'autorità: il governatore sarà infine costretto alla fuga e all'abbandono del governo del cantone di Valais. Berneri chiude qui la narrazione, probabilmente per lasciare intendere che di un'autorità si può fare a meno e che il moto rivoluzionario contro il potere possa partire in maniera spontanea e immediata da piccole unità territoriali capaci di aver ragione del potere costituito e di autogestirsi in maniera autonoma ed egualitaria. In altre parole, questo racconto potrebbe rappresentare il primo scritto berneriano in cui si manifestino idee comunaliste libertarie, anche se non nella forma di una riflessione politica vera e propria né tanto meno inquadrate all'interno di un compiuto modello di federalismo libertario. Il giovane Berneri, più appassionato e ancora ingenuo militante che non attento e meticoloso teorico, è ancora legato a uno scrivere e a un argomentare volto a divulgare e a rendere accessibile questa idea a quanti più lettori e militanti possibile. Per questo probabilmente sceglie e predilige la forma della piccola novella rispetto a quella dell'articolo o del saggio critico - con ogni probabilità è qui ravvisabile un'ulteriore influenza della madre Adalgisa Fochi e della sua produzione letteraria favolistica ${ }^{101}$.

Si tratta, riteniamo, di una prima e isolata manifestazione di un'idea che andrà nel tempo diventando uno dei cardini della riflessione politica berneriana.

\section{Un giovane anarchico tra guerra e rivoluzione}

Giunto all'anarchismo in cerca di una maggiore coerenza tra teoria e pratica e di un'opposizione più netta e decisa alla guerra, Berneri prosegue negli anni del conflitto sulla strada dell'antimilitarismo, tentando di fondare a Reggio Emilia un gruppo giovanile anarchico, prima tra gli studenti e in seguito tra gli operai. I rapporti di polizia segnalano il suo attivismo e affermano che egli «può considerarsi per la sua attività, intelligenza e fermezza elemento pericoloso ${ }^{102}$. Le sue iniziative

${ }^{100} \mathrm{C}$. Berneri, Novella storica cit.

${ }^{101}$ Cfr. C. De Maria, Una famiglia anarchica cit., pp. 24-25.

${ }^{102}$ Prefettura di Reggio Emilia, Cenno biografico cit. 
non avranno successo, ma ciò non gli impedisce di prendere parte a importanti mobilitazioni promosse all'interno del movimento anarchico attraverso la sua amicizia con Gobbi. L'intervento dell'Italia nella Grande Guerra aveva portato un oppressivo e pervasivo clima di repressione e di controllo in tutta la penisola che aveva in particolar modo colpito i movimenti a carattere sovversivo, anarchici in testa. Il movimento si trova di fatto a operare nella semi-clandestinità, tuttavia il 27 giugno 1916 riesce a tenere un Convegno a Firenze cui partecipa un nutrito numero di individualità, gruppi e sezioni. Durante il Convegno viene fondato un Comitato d'Azione Internazionale Anarchico (CAIA) col compito di coordinare il frammentato movimento anarchico italiano e di sviluppare collegamenti internazionali nell'opposizione alla guerra ${ }^{103}$. Torquato Gobbi è uno dei delegati del CAIA e ha in particolare il compito di mantenere i rapporti tra Emilia-Romagna e Lombardia; Berneri sarà al suo fianco in questa attività ${ }^{104}$.

Il 1916 coincide anche col trasferimento della madre e del giovane anarchico ad Arezzo, costringendo quest'ultimo a lasciarsi alle spalle Reggio Emilia, forse la prima città in cui ha veramente messo delle radici. Nella città toscana Berneri completa gli studi liceali, recuperando a ottobre le lacune in latino, greco e matematica, ma soprattutto trova un ambiente anarchico più vivace e numericamente nutrito rispetto a quello reggiano: frequenterà gli anarchici aretini del Circolo di studi sociali «I Liberi» e gli ambienti sindacalisti, progetterà anche la fondazione di un quindicinale in cui raccogliere le voci della gioventù sovversiva che avrebbe dovuto chiamarsi «La Giovane Internazionale» - nel quale avrebbero dovuto trovare voce tanto le questioni culturali atte a preparare ed educare i militanti quanto quelle di lotta e battaglia sindacale ${ }^{105}$.

\section{La chiamata alle armi}

Il 10 gennaio 1917 Berneri sposa Giovanna Caleffi e il 1 marzo dell'anno successivo nasce la loro prima figlia, Maria Luisa. La Grande Guerra però non tarda a bussare alla porta del giovane Berneri che, il 15 marzo 1917, è chiamato a prestare servizio militare e destinato al Deposito del Genio a Casale Monferrato. Approfitterà inizialmente di un permesso speciale per frequentare la Facoltà di Filosofia e Filologia presso il Regio Istituto di Studi Superiori di Firenze, ma il 30 ottobre deve trasferirsi a Modena presso la Regia Accademia Militare dove, in quanto diplomato, frequenta il corso obbligatorio per la nomina ad aspirante ufficiale. Berneri, da oppositore alla guerra e all'esercito quale ha sempre dimostrato di essere, appare

\footnotetext{
${ }^{103}$ Vedi F. Giulietti, Gli anarchici italiani dalla grande guerra al fascismo, Franco Angeli, Milano, 2015, pp. 43-49.

${ }^{104} \mathrm{Si}$ vedano le note della Prefettura di Reggio Emilia, 28 dicembre 1916 e della Prefettura di Varese, 11 gennaio 1917 in ACS, Min. In., CPC, Berneri Camillo cit.

${ }^{105}$ Vedi Circolare Annuncio del quindicinale «La Giovane Internazionale», periodico quindicinale: organo della gioventù anarchica, in appendice a C. Berneri, Epistolario inedito, vol. II, a cura di Paola Feri e Luigi Di Lembo, Edizioni Archivio Famiglia Berneri, Pistoia, 1984, pp. 251-252. Sugli anni trascorsi da Berneri ad Arezzo e la sua attività vedi G. Sacchetti, Le culture politiche del giovane Berneri cit., pp. 36-42.
} 
però fin da subito insofferente agli obblighi della leva e anzi vi prosegue l'attività che più di tutte sta caratterizzando questa sua fase di militanza, ossia la propaganda antimilitarista e anarchica. Ciò gli costerà il trasferimento dall'Accademia al Genio in Casale Monferrato, Secondo Reggimento Zappatori: verrà spedito al fronte e ci resterà fino alla fine del conflitto, venendo ferito e due volte denunciato al Tribunale di guerra.

Durante la guerra proseguono, anche se si fanno più rade, le collaborazioni di Berneri con la stampa anarchica. Le prime notizie che arrivano dalla Russia rivoluzionaria stanno accendendo gli animi e gli entusiasmi del movimento anarchico, nel mentre si avvia un vivace dibattito sugli avvenimenti e sugli auspici che si ripongono nell'esperienza russa. Anche l'attenzione e le speranze di Berneri si rivolgono verso la lontana Rivoluzione Russa, come sottolineano molti studi sull'anarchico lodigiano che però si sono prevalentemente concentrati sul contenuto delle sue ricerche e sulle opinioni da lui espresse circa la rivoluzione, passando in rassegna i contenuti e gli argomenti principali da lui sviluppati con riguardo alla situazione russa ${ }^{106}$. Noi ci soffermeremo piuttosto su come Berneri faccia riferimento agli eventi rivoluzionari che stanno avvenendo in Russia per recuperare quello che è stato il motivo principale che lo ha portato a rompere con il PSI, riproponendo così la questione polemica sullo scarso attivismo dei socialisti e tentando allo stesso tempo di spingere sul terreno rivoluzionario quanti di loro, soprattutto nelle file giovanili, si sentissero costretti e limitati all'interno della linea politica adottata dal proprio partito.

Un primo interessante scritto sul tema, pubblicato su «Guerra di Classe», è volto per l'appunto a sottolineare la presenza e il ruolo attivo e combattivo degli anarchici russi negli eventi rivoluzionari passati e recenti del proprio paese, denunciando per contro il silenzio della stampa democratica e socialista su questo aspetto: il timore del giovane anarchico, che deriva senza dubbio dagli strascichi del dibattito sull'interventismo cui aveva preso parte già durante la militanza nella FIGS, è che la rivoluzione passi «come una vittoria degli intesisti democratici» e non come un fenomeno cui hanno preso parte «forti e numerose correnti neutraliste e realmente rivoluzionarie» in cerca di attuare il proprio programma massimo ${ }^{107}$. Si può notare come la contrapposizione messa in rilievo sia tra interventisti e anti-interventisti che, lo ricorderemo, aveva infine portato Berneri a fuggire dalle incerte posizioni del Partito Socialista per prendere la strada dell'anarchismo. Non dev'essere quindi un caso trovare qualche mese dopo, sempre su «Guerra di Classe», un altro articolo di Berneri rivolto stavolta ai suoi ex-compagni di strada, i giovani socialisti ${ }^{108}$ : privi di ogni autonomia che non riguardi il semplice proselitismo e l'attività culturale, costretti alla passività e in preda al dottrinarismo della dirigenza, i militanti della

\footnotetext{
${ }^{106} \mathrm{Si}$ veda in particolare G. Carrozza, En Russie bolscheviste. L'analisi di Berneri sull'Unione Sovietica all'inizio degli anni '20, in Un libertario in Europa cit., pp. 51-70.

${ }^{107}$ C. Berneri, Per un silenzio ingiusto, «Guerra di Classe», III, 22 aprile 1917. L'articolo, col titolo Gli anarchici e la rivoluzione russa, è riprodotto in Id., Pietrogrado 1917 Barcellona 1937 cit., pp. 15-18.

${ }^{108}$ Id., Mentre i giovani socialisti polemizzano, «Guerra di Classe», III, 30 giugno 1917.
} 
FIGS non riescono, agli occhi del giovane anarchico, a sviluppare la propria critica che potrebbe riportare il partito alla sua vera natura rivoluzionaria. Chiudendo l'articolo, ancora una volta Berneri cerca di chiamare a raccolta $i$ giovani socialisti per far nascere un nuovo blocco di forze: «Ci sono dei giovani socialisti che sono con noi e con la nostra preparazione spirituale per un domani di lotta? Se sì, si facciano avanti!» ${ }^{109}$.

In seguito Berneri decide di portare la contrapposizione col Partito Socialista agli estremi con un articolo deciso e pungente nell'imporre un aut-aut tra Kerenskij e Lenin, le due anime della rivoluzione: «Si è con Kerenskij, col dittatore intesista, juscaborista, o si è con Lenin, internazionalista, rivoluzionario?» ${ }^{110}$. Egli denuncia la posizione ambigua del PSI, che di fronte all'evidente e radicale contrapposizione tra i programmi dei due personaggi, dovrebbe preoccuparsi di precisare in maniera netta i propri atteggiamenti e le proprie tendenze. Di nuovo, la Rivoluzione Russa diventa una cartina di tornasole per saggiare la natura e le intenzioni di un partito in cui appare sempre più chiaro, secondo Berneri, il dissidio tra una base sinceramente rivoluzionaria e una dirigenza ambigua, attendista; non si dimentichi che proprio questa era stata una delle questioni che aveva allontanato il giovane anarchico dalla FIGS, spingendolo a scrivere quella lettera di dimissioni in cui invitava i giovani socialisti scontenti del partito a seguirne l'esempio. Di nuovo, quello di Berneri è un appello all'azione, alla mobilitazione delle masse, al coraggio di mettersi in campo con la fede e la volontà, "gli Amleti debbono essere lasciati agli eterni dubbi dottrinari» ${ }^{111}$.

Può sembrare strano che un anarchico prenda le parti di Lenin, leader dei bolscevichi e futuro soppressore di anarchici, ma bisogna considerare che non solo Berneri, ma tutto il movimento anarchico in questo frangente si stia trovando a prendere le parti dei sostenitori delle tesi leniniste piuttosto che quelle di Kerenskij. In parte ciò è dovuto al fatto che le notizie che giungono dalla Russia siano discontinue, frammentarie e spesso contraddittorie, ma a ciò va soprattutto aggiunta la campagna diffamatoria condotta dalla stampa cosiddetta borghese dell'esperienza rivoluzionaria, che porta spontaneamente gli anarchici italiani a solidarizzare con le forze radicali e rivoluzionarie russe, bolscevichi inclusi: la decisione e la radicale fermezza di questi ultimi non possono così che incontrare il plauso di buona parte del movimento che, anche se vede bene che il loro indirizzo rivoluzionario non ha certo un carattere anarchico, ritiene comunque che con esso si possano quanto meno aprire prospettive libertarie. Solamente a partire dal 1918 il movimento anarchico inizierà a oscillare tra prime riserve e residuali solidarietà, tra critiche e difese della

\footnotetext{
${ }^{109}$ C. Berneri, Mentre i giovani socialisti polemizzano, «Guerra di Classe», III, 30 giugno 1917.

${ }^{110}$ Id., Con Kerenskij o con Lenin, «Guerra di Classe», III, 6 ottobre 1917. L'articolo è riprodotto in Id., Pietrogrado 1917 Barcellona 1937 cit., pp. 19-23, e in Id., Scritti scelti cit., pp. 49-53.

${ }^{111}$ Ibid.
} 
leadership bolscevica, mentre le disillusioni e l'opposizione si faranno più forti e decise solo nel corso degli anni ' $20^{112}$.

\section{Una collaborazione con dei repubblicani "eretici"}

Ci sembra evidente che, mentre parla di Rivoluzione Russa, la speranza di Berneri sia quella di poter ricostituire un blocco di forze politiche sovversive e antimilitariste similare a quello che si mobilitò al tempo della Settimana Rossa, ma anche quella di spingere il Partito Socialista ad assumere una posizione più forte $\mathrm{e}$ meno legalitaria, unico vero ostacolo a un eventuale congiungimento di movimenti e partiti politici contrari alla guerra e votati alla rivoluzione sociale ${ }^{113}$. Da questo punto di vista è interessante rilevare come le attenzioni e la collaborazione del giovane anarchico si rivolgano anche a un eccentrico gruppo di repubblicani, altra importante componente di quella storica opposizione insurrezionale alla guerra.

Gli studi e i saggi critici su Berneri finora pubblicati non si sono particolarmente soffermati sui suoi articoli comparsi su «Il Grido», quindicinale di critica sociale edito a Napoli tra 1917 e 1918 e animato da Dino Fienga ${ }^{114}$, giovane repubblicano a capo di una corrente di militanti che si era opposta alla scelta interventista del Partito Repubblicano Italiano $(\mathrm{PRI})^{115}$. È invece necessario oltreché interessante analizzare questa testata estremamente particolare cui Berneri decide di prestare la propria penna, in quanto costituisce la prima e tangibile testimonianza dell'interesse

${ }^{112}$ Sull'atteggiamento degli anarchici italiani di fronte alla Rivoluzione Russa resta indispensabile il riferimento a S. Fedele, Una breve illusione: gli anarchici italiani e la Russia sovietica, 1917-1939, Franco Angeli, Milano, 1996, ma si veda anche il più recente F. Bertolucci, A Oriente sorge il sol dell'avvenire. La rivoluzione russa vista dagli anarchici italiani 1917-1922, Edizioni Biblioteca Franco Serantini, Pisa, 2017. Cfr. inoltre P. C. Masini, Gli anarchici italiani e la rivoluzione russa, «Rivista storica del socialismo», V, $\mathrm{n}^{\circ} 15-16$, giugno-agosto 1962, pp. 135-169 e F. Giulietti, Gli anarchici italiani dalla grande guerra al fascismo cit., pp. 57-84.

${ }^{113} \mathrm{Si}$ veda a tal proposito C. Berneri, Fuori dal partito socialista non v'è azione socialista?, «L'Avvenire Anarchico», VIII, 26 ottobre 1917, in cui Berneri torna a criticare la disciplina interna al PSI e la perdita del suo carattere rivoluzionario che impediscono la possibilità di un dialogo con anarchici e sindacalisti.

${ }^{114}$ Bernardino "Dino" Fienga (1893-1975) fu un medico e, più tardi, bibliotecario salernitano. Militante repubblicano, egli passa in seguito al socialismo da cui si staccherà nel 1921 per aderire al Partito Comunista d'Italia. Sulla sua vicenda non vi sono numerosi studi, tuttavia si può vedere «Fienga, Bernardino (Dino)», in G. Vaccaro (a cura di), Panorama biografico degli italiani d'oggi, vol. I, Curcio, Roma, 1956, p. 625. Cfr. inoltre la scheda di F. Tuccillo, Il caso Fienga: le testimonianze di una vita straordinaria sul sito della Biblioteca Nazionale di Napoli < http://www.bnnonline.it/index.php?it/158/il-caso-fienga-le-testimonianze-di-unavita-straordinaria $>(09 / 2019)$.

${ }^{115} \mathrm{Si}$ veda l'articolo redazionale Il Grido, Schiarimenti. Per intenderci, «Il Grido», I, 20 novembre 1917, in cui vengono esplicitati i sentimenti anti-interventisti degli animatori del periodico e la loro intenzione di fare critica e opposizione all'interno del PRI per ricondurlo verso le masse e sulla questione sociale. 
berneriano per l'apertura e il dialogo verso movimenti o correnti di partito "irregolari" e individualità "eretiche", in cerca non soltanto di interlocutori ma anche di possibili alleati con cui condividere idee e battaglie politiche in prospettiva rivoluzionaria. Sono passati poco più di tre anni dall'esperienza della Settimana Rossa e di certo il ricordo ancora vivo di quell'episodica convergenza di forze capace di contagiare e di mettere in agitazione parte della penisola, seppur per un limitato periodo di tempo - deve aver convinto Berneri che solo condividendo il cammino, o parte del cammino, con altre forze politiche sia possibile raggiungere risultati concreti.

Fienga e i collaboratori del periodico sembrano, da questo punto di vista, perfettamente rispondenti al profilo di interlocutori cui il giovane anarchico può essere interessato. In primo luogo essi insistono sulle tendenze presenti nel pensiero repubblicano che auspicano la rivoluzione sociale e l'emancipazione del proletariato $^{116}$, intendendo trovare un punto di incontro tra repubblicani e altre forze rivoluzionarie. Tra i punti del loro programma si possono inoltre notare la volontà di un «affratellamento, ma senza confusioni come senza urti, dei libertari tutti (repubblicani, socialisti, anarchici) per un concorde lavoro di educazione popolare, che renda possibile l'esperimento delle più ardite riforme politiche ed economiche», l'opposizione «ad ogni specie di collaborazione con gli attuali partiti costituzionali, pur non essendo per l'astensionismo elettorale e parlamentare», e l'intenzione di attuare un'azione politica «di critica e di opposizione alle vigenti istituzioni» ${ }^{117}$. Altro aspetto interessante da sottolineare è il fatto che Fienga e gli animatori de «Il Grido» qualifichino se stessi, gli anarchici e i socialisti come libertari, termine che generalmente si è soliti adoperare come sinonimo per i soli anarchici. Per la verità l'aggettivo era allora un termine ancora estremamente generico in cui si poteva riconoscere chiunque si opponesse genericamente all'autorità, allo statalismo, al centralismo e all'oppressione, solamente a inizio Novecento è riscontrabile nel vocabolario di parte degli anarchici che intendevano abbandonare la vecchia definizione di socialismo anarchico ${ }^{118}$. Inoltre l'accento messo da questi repubblicani sui generis sulla questione sociale, la loro critica radicale alle istituzioni monarchiche, da cui deriva il rifiuto di collaborare coi partiti costituzionali, e infine la loro ferma e risoluta opposizione alla guerra sono tutte caratteristiche che devono certo aver riscosso l'interesse di Berneri, richiamando alla sua mente quella famosa koiné presente non solo nella sua educazione politica, ma che era stata anche interpretata e condivisa da quei repubblicani di sinistra, anarchici e socialisti che si erano ritrovati uniti nel 1914 durante la Settimana Rossa.

\footnotetext{
${ }^{116} \mathrm{Negli}$ articoli e nelle citazioni che vengono riportate in grassetto sul quindicinale ricorrono spesso i nomi di Bovio, Pisacane, Cattaneo e Ferrari, ma non mancano riferimenti anche al pensiero anarchico di Kropotkin.

${ }^{117}$ [D. Fienga], Repetitia iuvant, «Il Grido», II, 5 gennaio 1918, il corsivo è nostro. Nella conclusione dell'articolo viene anche dichiarato che gli animatori del periodico sono impegnati nella costruzione di un «Gruppo Socialrepubblicano» all'interno del PRI.

${ }^{118}$ Vedi a tal proposito P. C. Masini, Storia degli anarchici italiani nell'epoca degli attentati, Rizzoli, Milano, 1981, p. 178.
} 
L'interpretazione dell'idea di repubblica data dai collaboratori de «Il Grido» è peraltro estremamente peculiare, come dimostra l'articolo del socialista "irregolare" Alfonso Leonetti intitolato L'unicità del mezzo: secondo l'autore non solo «la concezione repubblicana è la sola vera che possa darci una forma di governo libertario», ma addirittura «socialismo e anarchismo sono teoriche repubblicane»" ${ }^{119}$. Tale posizione è giustificata a partire da un'interpretazione radicale e alquanto originale del termine res publica che, secondo l'autore, starebbe per «una società in cui tutto avesse carattere pubblico» e in cui vi sarebbe pertanto, in ultima istanza, «assenza di ogni capo» ${ }^{120}$. In altre parole si potrebbe dire che, secondo Leonetti, la repubblica sia non solo una condizione pregiudiziale o un momento intermedio nella realizzazione della libertà individuale e della giustizia sociale, ma anche e soprattutto il fondamento stesso da cui deriverebbero e a cui sarebbero fortemente legate le idee socialiste e anarchiche. Forzando un po' la mano, Leonetti sta cercando di declinare l'antica koiné dei rivoluzionari in modo che essi possano parlarsi, comprendersi, unirsi in una piattaforma d'azione comune e, perché no, riconoscersi accomunati dalle stesse radici politiche e ideali.

Appare evidente che lo spirito e la linea politica del quindicinale napoletano abbiano insomma più di un punto di contatto con gli interessi di Berneri e con la tattica che egli vorrebbe mettere in campo contro lo Stato e contro la guerra, favorendo così una svolta rivoluzionaria. È peraltro possibile che sia stato «Il Grido» a trovare Berneri e non viceversa, dato che nella rubrica «Emeroteca», in cui si recensiscono o commentano alcuni articoli scelti, erano stati segnalati nel corso del 1917 alcuni scritti del giovane anarchico ${ }^{121}$. Il 20 giugno 1918 compare invece il primo contributo di Berneri che, interamente imbiancato dalla censura, avrebbe dovuto essere intitolato I mazziniani e la guerra, probabilmente una riproduzione di un articolo dal titolo analogo già uscito sul settimanale pisano «L'Avvenire Anarchico», in cui Berneri critica il recupero di Mazzini in chiave interventista da parte dei repubblicani e la loro decisione di schierarsi nella guerra al fianco di una monarchia cui non avevano mai risparmiato critiche ${ }^{122}$.

${ }^{119}$ A. Leonetti, L'unicità del mezzo, «Il Grido», I, 5 dicembre 1917.

Alfonso Leonetti (1895-1984) è stato un politico aderente, fin da giovanissimo, al socialismo. Antimilitarista e dirigente del comitato del «Soldo al soldato», in seguito sarà tra i fondatori del Partito Comunista d'Italia. Sulla sua figura si rimanda a «Leonetti, Alfonso» in F. Andreucci e T. Detti (a cura di) Il movimento operaio italiano. Dizionario biografico, 18531943, vol. III cit, pp. 97-100.

${ }^{120} \mathrm{~A}$. Leonetti, L'unicità del mezzo cit.

${ }^{121}$ Vedi Emeroteca, «Il Grido», I, 5 novembre 1917 e 20 dicembre 1917: nel primo viene recensito l'articolo Da Kipling a Chamberlain pubblicato su «L'Avvenire Anarchico» nei numeri del 12 e del 26 ottobre 1917, nel secondo l'articolo Un pedagogista moderno. Jean Lightart pubblicato su «L'Università Popolare» nel numero del 15 dicembre 1917.

${ }^{122} \mathrm{C}$. Berneri, I mazziniani e la guerra monarchico-repubblicana, «L'Avvenire Anarchico», VIII, 17 agosto 1917. Berneri aveva anche pubblicato un altro articolo di tenore simile sul settimanale pisano, vedi Id., Mazzini e Bismarck, «L'Avvenire Anarchico», VIII, 29 giugno, 1917. 


\section{Asterischi e marionette}

Un altro articolo del giovane anarchico comparirà tre mesi dopo e tratterà dell'assenza di una filosofia e di una cultura adeguate a supportare e condurre le trattative di pace ${ }^{123}$ : secondo Berneri, in Italia il mondo degli intellettuali ha preferito mettersi al servizio della cultura dominante della guerra e dell'intervento, «meditando, scrivendo, parlando non al di sopra della mischia bensì nel folto di essa, sanzionando gli istinti più bassi, più primitivi, le ideologie più anacronistiche»» ${ }^{124}$. Richiamandosi a Kant e alla sua opera Per la pace perpetua (1795), il giovane anarchico vede nella filosofia del suo tempo tracce evidenti di debolezza e di malattia, l'incapacità di «svolgere un'azione innovatrice e perfezionatrice» se non all'interno di un circolo ristretto ed egemonizzato da un «Olimpo accademico» composto da «pochi pensatori appartenenti alle classi dominanti» che sono «per atavismo e per educazione ricevuta, figli del loro ambiente e del loro tempo» ${ }^{125}$. Secondo l'anarchico, le filosofie di Stirner, Nietzsche e del darwinismo sociale sono colpevoli di aver messo da parte il senso della solidarietà e della giustizia sociale, mettendo in atto «un meschino ed egoistico utilitarismo» dove «il bene egoistico trionfa sul bene altruistico a scapito del bene universale». Individuando nella confusa «amalgama di astruserie metafisiche, di sofismi, di idee ipercerebrali» la causa del decadimento della filosofia a lui contemporanea, Berneri auspica che il pragmatismo e il futuro dopoguerra possano portare con sé «una filosofia umana» ${ }^{126}$. Questo contributo, lontano per certi versi dall'usuale taglio degli scritti giovanili berneriani, ci mostra l'altro lato del giovane militante anarchico, quello più disponibile alla meditazione intellettuale profonda, certo alimentata dalle prime frequentazioni degli ambienti universitari. Berneri appare capace di far convivere il suo atteggiamento "culturista" con gli slanci rivoluzionari, in altre parole di far avanzare insieme pensiero e azione.

La critica alla cultura e al giornalismo italiano ritornano ancora nel suo ultimo contributo su «Il Grido», intitolato Le marionette storiche ${ }^{127}$. L'articolo è molto interessante perché, dopo essere stato a lungo - come molti altri anarchici - tra i sostenitori e i simpatizzanti di Lenin, per la prima volta Berneri afferma che sia necessario ridimensionare la crucialità della figura del leader bolscevico nella Rivoluzione Russa: il giovane anarchico nota come vi sia, in generale, la tendenza ad attribuire eccessivo peso e centralità ai grandi personaggi storici, mettendo in parallelo il modo in cui viene tratteggiata la figura di Lenin con quello con cui sono stati descritti e portati al pubblico altri personaggi quali Rasputin, Cagliostro, Robespierre, Masaniello e Napoleone. Secondo Berneri ciò non sarebbe altro che «la

${ }^{123}$ B. C. [C. Berneri], Asterischi. Della filosofia del dopoguerra, «Il Grido», II, 20 settembre 1918.

${ }^{124}$ Ibid.

${ }^{125}$ Ibid.

${ }^{126}$ Ibid.

${ }^{127}$ C. Berneri, Le marionette storiche, «Il Grido», II, 5 novembre 1918, riprodotto in Id., Pietrogrado 1917 Barcellona 1937 cit., pp. 24-29. 
manifestazione nuova di un vecchio errore» che tende a ridurre la storia alla biografia di personaggi illustri «trascurando correnti d'idee, il fattore economico e tutte le altre cause dinamiche poste in soffitta con Marx ed il materialismo storico» ${ }^{128}$. Così Lenin, nell'immagine che ne dà la stampa quotidiana e specialistica, diventa l'unico e solo protagonista del più complesso fenomeno rivoluzionario russo, un capro espiatorio sui cui gesti e parole si esercita uno «sciocco e partigiano sforzo di giudizi falsi e calunniosi»; più in generale, la critica storica assomiglia a un «teatro dei burattini» simile alla storia che viene insegnata a scuola «a furia di episodi stupefacenti, di bei gesti e di belle pose, di oleografia a forti tinte» ${ }^{129}$.

Questi ultimi due articoli mostrano un Berneri in fase di maturazione intellettuale, capace di elaborare profonde riflessioni critiche sulla cultura e la filosofia del suo tempo: questo mutamento è probabilmente un frutto spontaneo della sua crescita, alimentata senza dubbio anche dai corsi frequentati presso la Facoltà di Lettere immediatamente prima della partenza per il fronte. L'approccio adottato in questi due articoli è di un livello del tutto inedito nel Berneri di allora, ben più interessato come abbiamo visto all'attualità politica e all'urgenza di favorire e organizzare un nuovo movimento rivoluzionario. È più che probabile che lo sviluppo di riflessioni su questi temi derivi proprio dai primi studi e dalle prime lezioni di Pedagogia o di Storia della Filosofia svolti presso il Regio Istituto di Studi Superiori, sui quali finora c'è stata poca attenzione da parte degli studiosi e degli storici dell'anarchico lodigiano. Dalla consultazione della sua Carriera Studente, conservata presso la Sala Rari della Biblioteca Umanistica dell'Università degli Studi di Firenze, risulta che i corsi sopracitati siano in effetti i primi a essere stati registrati prima della sua partenza per il fronte ${ }^{130}$. È particolarmente meritevole di interesse l'articolo del 20 settembre 1918 sulla filosofia del dopoguerra in cui il giovane anarchico auspicava l'adesione a un più umano pragmatismo con cui spazzare via il fardello di idee e ordini di idee che gravano e confondono la mente dell'essere umano: non stupisce che proprio la corrente filosofica del pragmatismo mai comparsa prima negli scritti berneriani - abbia destato l'interesse del giovane studente, considerando la sua tendenza, fin dai tempi della sua prima militanza socialista, a lavorare sul concreto e a rifuggire le astrattezze.

L'11 novembre 1918 termina la Grande Guerra e così il quindicinale napoletano «Il Grido», nato per esprimere dissenso rispetto alla scelta interventista del PRI e alla guerra, sospende la propria pubblicazione per riportare la battaglia politica in seno al partito. L'avvicinamento di Berneri a questi repubblicani sui generis non sarà tuttavia priva di conseguenze, anzi la componente sociale e rivoluzionaria del PRI

${ }^{128} \mathrm{C}$. Berneri, Le marionette storiche cit.

${ }^{129} \mathrm{Ibid}$.

${ }^{130}$ Vedi Anno $1^{\circ}$, Studi, in Carriera Studente di Berneri, Luigi, p. 143, in ASUF, Regio Istituto di Studi Superiori, Pratici e di Perfezionamento in Firenze. Sezione di Filosofia e Filologia. Registro della carriera scolastica degli studenti X (1914-1917). Pur non essendo segnalati i nomi dei professori dei diversi corsi, è lecito ritenere che Berneri abbia seguito il corso di Pedagogia del prof. Calò e quello di Storia della Filosofia del prof. Melli, con cui seguirà successivamente anche il Corso d'Integrazione, vedi Anno $2^{\circ}$, Studi, in ibid. 
costituirà negli anni a venire uno dei punti di riferimento per il giovane anarchico in cerca di confronti e di intese con altre forze politiche. Pensiero e azione, come si può vedere, continuano a essere $\mathrm{i}$ due poli d'interesse e $\mathrm{i}$ due termini tra cui il giovane Berneri si trova a oscillare. Nel movimento anarchico gli sembra finalmente di aver trovato una maggiore coerenza tra la sua volontà rivoluzionaria e la pratica, un'opposizione più sincera e aperta a quello status quo che ai suoi occhi il PSI non sapeva - e non poteva né voleva - dare. In buona parte degli articoli di questa sua fase giovanile, come abbiamo visto, vengono espressi a chiare lettere i suoi tentativi di pungolare $\mathrm{i}$ socialisti verso una più diretta azione di rivendicazione che sia coerente coi propri principi di partenza, soprattutto in relazione all'infuriare del conflitto mondiale: in questo senso possono essere interpretati non solo i suoi primi articoli sulla Rivoluzione Russa che abbiamo analizzato, ma anche i suoi primi articoli sul sindacalismo che tendono a squalificare le posizioni "collaborazioniste" dei sindacati di ispirazione socialdemocratica col governo ${ }^{131}$, tornando sempre sulla necessità di rafforzare e di riportare su un terreno più concretamente rivoluzionario il movimento socialista.

Abbiamo detto che Berneri ha imparato fin dal suo primo apprendistato politico ad apprezzare gli impegni concreti e che ciò lo ha anche allontanato dalle vaghezze rivoluzionarie dei massimalisti, tuttavia abbiamo anche visto come abbia sempre inteso accompagnare il suo impegno con una fervida intenzione di realizzare un effettivo mutamento sociale. $\grave{E}$ dunque da ritenersi che, secondo il giovane anarchico, l'emancipazione delle classi subalterne necessitasse sì di un'opera di propaganda e preparazione culturale, ma che tale lavoro sarebbe risultato in ultima istanza perfettamente inutile se poi in campo politico e sindacale il PSI non avesse avuto il coraggio di difendere le stesse classi subalterne, opponendosi con decisione alla guerra o supportando la causa dei rivoluzionari russi contro i moderati. Ricordiamo anche il progettato e mai realizzato quindicinale «La Giovane Internazionale», un periodico della gioventù anarchica che avrebbe dovuto essere caratterizzato da contenuti sia culturali che propagandistici, insomma tanto dall'una e quanto dall'altra componente.

La carica ideale e l'adesione - più umanitaria e sentimentale che non analitica e scientifica - ai principi di uguaglianza sociale e di elevazione delle masse sono insomma ancora tra $\mathrm{i}$ dati caratteristici del giovane Berneri, dati che successivamente peraltro non scompariranno mai e anzi attraverseranno e qualificheranno ancora il suo pensiero politico. Accanto a questi aspetti è possibile però vederne emergere altri, che diventeranno altrettanto importanti e caratteristici del pensiero berneriano, ossia quelli dello studio e dell'analisi approfondita dei problemi e delle diverse questioni filosofiche, politiche e culturali che emergono dalla stampa e dal dibattito intellettuale del periodo.

${ }^{131}$ Vedi B. C. [C. Berneri], Tradeunionismo e sindacalismo in Inghilterra, "Guerra di Classe», III, 11 agosto 1917. 


\section{Capitolo 2 \\ Il federalismo per realizzare l'avvenire anarchico: la maturazione del pensiero politico di Camillo Berneri}

\section{Gli studi universitari e l'incontro con Salvemini}

Al termine del conflitto non è ancora giunto per Berneri il termine del suo obbligo di servizio, così viene aggregato in qualità di scritturale alla prima sezione del Deposito del terzo reggimento Genio telegrafisti presso Firenze. In attesa di essere congedato, si trasferisce con la famiglia in città, in viale Volta al civico 13, e può così riprendere nell'aprile del 1919 gli studi universitari. Giorgio Sacchetti ha individuato in questa fase «la cesura fondamentale del percorso politico berneriano», soffermandosi in particolar modo sul fatto che le frequentazioni fiorentine permetteranno al giovane anarchico di aprirsi e di dialogare maggiormente con altri movimenti ed esperienze politiche ${ }^{132}$. Va tuttavia messa almeno in altrettanta evidenza la crucialità del periodo fiorentino anche sotto un altro aspetto, vale a dire quello dello studio e della crescita culturale che accompagnano Berneri in quella città che ancora viveva del prestigioso titolo di «Atene d'Italia», dovuto al rilevante ruolo culturale e alle numerose associazioni e riviste qui sorte a cavallo tra Ottocento e Novecento ${ }^{133}$. Firenze è insomma un centro vivace, ricco di stimoli e di occasioni di crescita per una giovane mente come quella di Berneri, una mente che fino ad

${ }^{132} \mathrm{G}$. Sacchetti, Le culture politiche del giovane Berneri. Un intellettuale fra Arezzo, Firenze e Cortona, in G. Berti, G. Sacchetti (a cura di), Un libertario in Europa. Camillo Berneri: fra totalitarismi e democrazia. Atti del convegno di studi storici, Arezzo, 5 maggio 2007, Edizioni Biblioteca Panizzi, Archivio Famiglia Berneri-Aurelio Chessa in collaborazione con la Provincia di Arezzo, Reggio Emilia, 2010, pp. 42-46.

${ }^{133} \mathrm{Si}$ vedano a tal proposito L. Cerasi, Gli ateniesi d'Italia: associazioni di cultura a Firenze nel primo Novecento, Franco Angeli, Milano, 2000, e G. Luti, Firenze corpo 8. Scrittori, riviste, editori del '900, Vallecchi, Firenze, 1983. Cfr. inoltre A. Asor Rosa, La cultura, in Storia d'Italia, vol. IV, Dall'Unità a oggi, t. 2, Einaudi, Torino, 1975, pp. 1147-1158. 
allora si era misurata e formata all'interno di determinati ambienti politici e in contesti tutto sommato piccoli e provinciali.

Due grandi figure di organizzatori culturali come Prezzolini e Papini non possono dunque non esercitare un certo fascino sul giovane Berneri per via del loro porsi "fuori dal coro" rispetto alle idee dominanti e al conformismo culturale del tempo, per il loro essere provocatori battaglieri e contestatori iconoclasti, per la loro opera di promozione e diffusione di idee filosofiche e tendenze letterarie nuove, atte a sprovincializzare la cultura italiana. Berneri avrà modo di conoscere personalmente Prezzolini solo nel 1927, durante il suo esilio volontario in Francia, ricevendo da questi alcuni incarichi per la traduzione di un romanzo ${ }^{134}$. L'editore e giornalista vociano lo ricorderà, diverso tempo dopo, con queste parole:

Lo conobbi a Parigi e mi ha lasciato un'impressione profonda. Era un esemplare raro di umanità. Lo conobbi all'uscita di una conferenza commemorativa che avevo tenuto in un anfiteatro della Sorbona a Parigi per il Sesto Centenario della morte di Nicolò Machiavelli (1927). [...]

Mi pare che fosse stato professore di filosofia ed uno dei primi intellettuali a lasciare l'Italia causa la vittoria del Fascismo per stabilirsi a Parigi dove viveva con la famiglia l'esistenza difficile e precaria degli intellettuali sempre minacciati di sfratto o di prigione dalla polizia. Mi piaceva il modo con il quale raccontava le sue esperienze con i poliziotti di vari paesi, per scappare ai quali, quando lo arrestavano, si sottometteva tranquillamente, svignandosela qualche volta alla prima occasione che si presentasse. Certamente appariva nella conversazione di una cultura non comune, come poi mi son accorto dalle sue citazioni negli scritti che ho letto di lui ${ }^{135}$.

In un articolo del 1926 Berneri rievocherà invece il ricordo di un Papini che «volava, con audacia icarea verso cieli sconfinati» e dichiarerà:

Papini ci divertiva. Spezzava i lampioni filosofici e le vetrine letterarie con violenza di teppista geniale. Molte sue pagine spalancavano a noi, chiusi nel provincialismo della cultura sonzognana o pedantesca, finestre ampie, su strade popolate e contro cieli più vasti ${ }^{136}$.

L'esperienza de «La Voce», seppure esaurita, doveva essere certo nota al giovane anarchico anche in quanto parte di quello che Asor Rosa ha definito come il «blocco culturale antigiolittiano» ${ }^{137}$ : non è da escludere che egli possa averne

${ }^{134} \mathrm{Si}$ vedano a questo proposito le lettere inviate da Berneri a Prezzolini, riprodotte in C. Berneri, Epistolario inedito, vol. II, a cura di Paola Feri e Luigi Di Lembo, Edizioni Archivio Famiglia Berneri, Pistoia, 1984, pp. 34-36.

${ }^{135}$ G. Prezzolini, Prezzolini alla finestra, Pan Editrice, Milano, 1977, pp. 157-158.

${ }^{136}$ C. Berneri, L'uomo finito cattolico, «Fede!», IV, 21 aprile 1926.

${ }^{137}$ A. Asor Rosa, La cultura cit., p. 1353. Cfr. anche S. Lanaro, La cultura antigiolittiana, in I. Barbadoro (coordinata da) Storia della società italiana, vol. XX, Teti, Milano, 1981, pp. 427-464. 
apprezzato il carattere eccentrico, antiborghese - a tratti tendenzialmente anarcoide $^{138}$ - e l'opposizione intellettuale al sistema vigente, anche se di certo non poteva trovarvi interlocutori politici, sia per il carattere composito che impedì una sintesi delle diverse posizioni, sia per l'adesione all'interventismo tanto di Prezzolini quanto di Papini ${ }^{139}$. Gli studi su Berneri fanno troppo poco spesso cenno ai contatti che egli ebbe con questa area del mondo della cultura e in ogni caso non hanno mai posto in adeguato rilievo questo aspetto che invece noi riteniamo importante per capire quanto il giovane anarchico abbia, in questo periodo, avuto l'opportunità di arricchire la sua esperienza culturale guardando anche al di fuori degli ambiti della politica, del movimento anarchico e delle correnti rivoluzionarie in generale: non va dimenticato come «La Voce» abbia teso a rappresentare l'opposizione non solo al giolittismo ma, più in generale, alla burocrazia e al sistema politico italiano coevo, facendo anche episodici riferimenti al tema del decentramento e del regionalismo ${ }^{140}$.

L'incontro fondamentale di questo periodo è però quello con Gaetano Salvemini $\mathrm{e}$, anche in questo caso, è necessario mettere in rilievo che gli studiosi di Berneri raramente hanno dato a questa figura l'importanza che secondo noi merita. Il professore pugliese viene invero citato e riconosciuto come una delle influenze più importanti nella maturazione del pensiero berneriano, ma ad oggi nessuno ha indagato quanto profondamente il rapporto con Salvemini abbia favorito - quanto meno nei primi anni universitari - un apprezzabile mutamento nell'atteggiamento intellettuale del giovane anarchico; in altre parole, il riconoscimento dell'influenza salveminiana è stato fin qui episodico e generico, quando non superficiale, limitato spesso a rapidi cenni o a poche righe di commento nei diversi studi e saggi critici ad oggi esistenti: trattato in generale in maniera troppo sbrigativa e accennata, l'incontro con Salvemini, il suo pensiero, la sua attività e il peso che hanno avuto per Berneri finiscono così per restare marginali ${ }^{141}$. Il solo Claudio Strambi, nel suo ultimo lavoro, ha messo in qualche evidenza le influenze e i prestiti salveminiani presenti nella maturazione berneriana dei primi anni '20, ma ancora una volta è

\footnotetext{
${ }^{138}$ Lo stesso Prezzolini, nel citato ricordo di Berneri, dichiara: «Fin da giovane avevo letto molto degli anarchici e per un certo tempo m'ero considerato anch'io un anarchico [...]. In un certo senso mi direi anche ora un anarchico, se avessi la fede che molti anarchici hanno nella bontà naturale degli uomini». Vedi G. Prezzolini, Prezzolini alla finestra cit., p. 158.

${ }^{139}$ Sulle caratteristiche de «La Voce» vedi A. Asor Rosa, La cultura cit., pp. 1254-1264, ma anche A. Romanò (a cura di), La cultura italiana del '900 attraverso la riviste, vol. III, «La Voce» (1908-1914), Einaudi, Torino, 1960.

${ }^{140}$ Vedi a questo proposito U. Chiaramonte, Il dibattito sulle autonomie nella Storia d'Italia (1796-1996). Unità-federalismo-regionalismo-decentramento, Franco Angeli, Milano, 1998, pp. 584-587.

${ }^{141}$ Lo studioso più attento all'indagine sull'influenza salveminiana e capace di andare al di là di generici riferimenti è stato, ci sembra, F. Madrid Santos, Camillo Berneri, un anarchico italiano (1897-1937). Rivoluzione e controrivoluzione in Europa (1917-1937), Edizioni Archivio Famiglia Berneri, Pistoia, 1985, soprattutto alle pp. 118-121.
} 
mancato, a nostro avviso, un approfondimento mirato e puntuale su questo $\operatorname{aspetto~}^{142}$.

$\mathrm{Ci}$ proponiamo dunque in questa ricerca non solo di dare maggiore rilievo all'influenza del professore pugliese, ma anche di sostenere parte della nostra tesi su un'evidenza che ci pare innegabile: la lotta per rinnovare l'anarchismo e l'elaborazione originale di una teoria federalista libertaria da parte di Berneri devono molto al confronto e allo scambio con Salvemini avvenuti a partire dal loro primo incontro presso il Regio Istituto di Studi Superiori di Firenze. Di più, il loro stesso incontro assume le caratteristiche di un vero e proprio punto di svolta poiché, proprio da allora e non prima, gran parte degli scritti e delle riflessioni berneriane si rivolgeranno ad alcuni temi come l'autonomismo e il federalismo, la critica al centralismo burocratico, la critica dell'immobilismo degli anarchici, lo studio e la soluzione tecnica dei problemi, l'interesse per motivi e tradizioni politiche non anarchiche che possono però arricchirne e renderne più complesso il pensiero e l'elaborazione teorica.

\section{Il magistero salveminiano}

Cominciamo però dal principio, ossia dal primo incontro tra Berneri e Salvemini che quest'ultimo ricorda con queste parole:

Berneri venne a trovarmi nel 1919 , cioè nell'immediato dopoguerra, vestito da soldato. Poche volte ho visto un soldato più scalcinato di quello. Pallido, magro, timido, parlava a bassa voce. [...] Ed era allora un'anima candida, dolce, desiderosa di lealtà e di affetto. Nei suoi occhi chiari, grigi si leggeva la bontà. Le idee politiche, come l'amore e come la tosse, non si possono nascondere. Mi disse che era anarchico. Poco male. Purché fosse stato sincero e intelligente, per me poteva essere tutto quello che voleva ${ }^{143}$.

In un'altra occasione Salvemini aveva inoltre scritto:

Siccome era la prima volta che avevo un anarchico fra i miei allievi, mi interessai a lui in maniera particolare.

Divenne uno degli studenti che venivano spesso a passare le loro serate con me, discutendo dei loro studi, del loro avvenire, delle questioni del giorno, di tutto ciò che ci poteva interessare ${ }^{144}$.

${ }^{142}$ Cfr. C. Strambi, L'inquieta attitudine: Camillo Berneri e la vicenda politica dell'anarchismo in Italia. Secondo Libro, "Il biennio rosso e rossonero", Edizioni Kronstadt, Pisa, 2017, specialmente alle pp. 219-220 e 246-248

${ }^{143}$ G. Salvemini, Donati e Berneri, «Il Mondo», IV, 3 maggio 1952, riprodotto parzialmente in Id., Opere VIII, Scritti vari (1900-1957), Feltrinelli, Milano, 1978, pp. 95-102.

${ }^{144}$ Dichiarazione di Gaetano Salvemini in favore di Camillo Berneri (13 agosto 1930), in C. Berneri, Epistolario inedito, vol. I, a cura di Aurelio Chessa e Pier Carlo Masini, Edizioni Archivio Famiglia Berneri, Pistoia, 1980, p. 134. 
Il rapporto tra Berneri e Salvemini non è dunque semplicemente il rapporto che si instaura tra studente e professore, tra allievo e maestro, ma è un vero e proprio rapporto di scambio e di confronto. Possiamo immaginare che l'incuriosito professore pugliese si sarà fatto raccontare dallo studente i motivi della sua adesione al movimento anarchico e il percorso intellettuale che lo ha condotto a tale decisione; seppure da prospettive diverse, già su questo aspetto i due potrebbero aver trovato un primo importante punto in comune: entrambi sono degli ex iscritti al PSI ed entrambi, seppur con motivazioni e auspici differenti, si sono distaccati dal partito criticandone la passività riformista e la conseguente tattica parlamentare. È altrettanto possibile che durante queste prime frequentazioni Salvemini abbia esposto a sua volta a Berneri le idee che lo avevano portato a scindersi dal Partito Socialista e dal gruppo redazionale de «La Voce» ${ }^{145}$, tentando di costituire un'aggregazione di intellettuali capaci di elaborare un rinnovamento politico e di favorire un'educazione civile attraverso idee e soluzioni concrete ai grandi problemi nazionali, in altre parole alternative credibili da opporre non soltanto ai programmi di moderati e conservatori, ma anche e soprattutto a quello socialista. Berneri sarà così venuto a sapere del settimanale fondato dal professore pugliese nel 1911, «L'Unità», altra rivista inquadrabile nel blocco culturale antigiolittiano e che lo studente anarchico però sentirà più vicina alle sue inclinazioni per i motivi che illustreremo.

Il periodico salveminiano è stato definito come «un coraggioso "che fare" della democrazia» ${ }^{146}$, un tentativo di spingere la politica su battaglie di interesse comune più concrete e immediate, lottando contro aspirazioni e dottrinarismi generici, verificando e mettendo alla prova principi, metodi e posizioni ideali, favorendo insomma «il richiamo alle cose e la demolizione di ogni avvenirismo» ${ }^{147}$. La filosofia del gruppo orbitante intorno a Salvemini, il cosiddetto «concretismo» o «problemismo», era stata a lungo discussa ed elaborata sulle colonne de «L'Unità» fin dalla sua presentazione sotto forma di lettera circolare:

Ciò che noi rimproveriamo ai partiti popolari - e non meno che agli altri al Partito Socialista - non è il loro ideale astratto di elevazione delle classi inferiori, ma la inettitudine a tradurre queste aspirazioni generiche in serie riforme concrete di utilità nazionale, - è la loro incapacità a dominare e coordinare gli appetiti e gl'interessi dei gruppi locali e delle categorie professionali in vista degli interessi collettivi, - è la impreparazione e l'assenteismo assoluto che la più parte dei politicanti dimostra di

\footnotetext{
${ }^{145}$ A questo proposito si veda A. Roveri, Salvemini, le grandi riforme e $i$ contadini meridionali, in I. Barbadoro (coordinata da), Storia della società italiana, vol. XX cit., pp. 283-299.

${ }^{146} \mathrm{~F}$. Golzio e A. Guerra (a cura di), La cultura italiana del '900 attraverso le riviste, vol. V, "L'Unità», «La Voce Politica» (1915), Einaudi, Torino, 1962, p. 17.

${ }^{147}$ Ivi, p. 36. Sulla filosofia che ispira il Salvemini e che farà da linea editoriale al settimanale cfr. inoltre G. De Caro, Gaetano Salvemini, Utet, Torino, 1970, pp. 101-104, e M. L. Salvadori, Gaetano Salvemini, Einaudi, Torino, 1963, pp. 82-86.
} 
fronte a tutti i problemi amministrativi, doganali, tributari, scolastici, internazionali, la cui soluzione urge al nostro paese $[\ldots]^{148}$.

Nel 1913 la questione veniva ulteriormente precisata in questi termini:

Che nella politica italiana d'oggi più che alle parole e alle formule su cui affermano di dividersi i partiti, si debba badare all'azione concreta dei partiti stessi [...]; e che una democrazia, la quale voglia esser democrazia sul serio e non per burla, abbia oggi soprattutto il dovere di definire chiaramente la sostanza delle riforme da proporre e da propugnare anzi che le formule astratte di partito nelle quali ammantarsi, - sono concetti che L'Unità ha predicato si può dire dal giorno in cui è sorta. E ha denunciato senza tregua la vanità e le insincerità, tanto delle vecchie ideologie popolariste bloccarde e confusionarie, quanto il bizantinismo delle suddivisioni tra rivoluzionari, riformisti sinistri, riformisti destri, in cui si esaurisce tutta la non attività pratica del Partito socialista ${ }^{149}$.

Rivolgendosi in particolare alla polemica tra riformisti e massimalisti all'interno del PSI, la denuncia si era ripetuta:

[...] e se voi vi limitate a predicare agli uomini le sole finalità astratte, senza indicar loro che cosa di concreto devono in vista di queste finalità desiderare giorno per giorno, i vostri uomini abbandonati senza guida nella vita economica e politica giornaliera, faranno [...] quello stesso opportunismo localista e babelico, che han fatto nel decennio passato $[\ldots]^{150}$.

Quando Berneri entra in contatto col Salvemini la maturazione e l'esposizione del concretismo di quest'ultimo è dunque giunta all'apice, oltre a costituire oramai la cifra dell'impegno di rinnovamento politico e civile che egli cerca di promuovere attraverso il suo settimanale. L'incontro tra $i$ due avviene peraltro in un periodo particolare in cui «L'Unità» sta esercitando una più larga influenza e le pressioni per farla diventare gruppo politico si stanno concretizzando e si iniziano a discutere le linee programmatiche della «Lega democratica per il rinnovamento della politica nazionale ${ }^{151}$. Si tratta invero di un'antica questione: già buona parte dell'annata del 1913 era stata occupata da un dibattito tra alcuni redattori desiderosi di andare oltre l'impostazione filosofica della rivista e di darsi un vero e proprio programma, un dibattito in cui Salvemini si era mostrato però sempre poco propenso a trasformare il

\footnotetext{
${ }^{148}$ G. Salvemini, Presentazione [de "L'Unità"], in Id., Opere IV, Il Mezzogiorno e la democrazia italiana, vol. I, Il ministro della mala vita e altri scritti sull'Italia giolittiana, Feltrinelli, Milano, 1962, p. 251.

${ }^{149}$ [G. Salvemini], L'astrattismo del concretismo, «L'Unità», II, 31 gennaio 1913, riprodotto in Id., Opere VIII cit., pp. 402-404.

${ }^{150}$ [G. Salvemini], Il nostro concretismo, «L'Unità», II, 28 febbraio 1913.

${ }^{151} \mathrm{Si}$ veda a tal proposito F. Grassi, Salvemini e l'ultima «Unità», in G. Cingari (a cura di), Gaetano Salvemini tra politica e storia, Laterza, Roma-Bari, 1986, pp. 329-353.
} 
gruppo de «L'Unità» in un movimento o in un partito ${ }^{152}$. Nel 1919, all'indomani della Grande Guerra, paiono profilarsi invece le condizioni per la nascita di una nuova democrazia e si lavora dunque alla realizzazione dell'antico progetto; tra i problemi più caldi che vengono discussi ci sono quello delle autonomie locali, del federalismo e della critica al centralismo burocratico, tutti temi a cui Salvemini è sempre stato sensibile ${ }^{153}$.

Non può essere un caso allora che uno dei primi articoli in cui Berneri inizia la propria riflessione sul decentramento e sulle possibili soluzioni atte a favorire forme di autogoverno e di autogestione da parte dei cittadini avvenga proprio in questo periodo, in quello che è da ritenersi - specialmente ai fini della presente ricerca uno degli articoli più importanti e interessanti all'interno della sua prima maturazione politica.

\section{Studiare la storia per riflettere sulla rivoluzione}

Lo scritto L'autodemocrazia appare su «Volontà», quindicinale anarchico di Ancona, il primo giugno del $1919^{154}$, ma l'articolo era già uscito in forma lievemente diversa nel settembre dell'anno precedente in "Guerra di Classe» ${ }^{155}$. Nessuno studio o saggio ha però fino ad oggi notato che in questo articolo emergano, per la prima volta in uno scritto berneriano, non solo evidenti influenze salveminiane ma anche citazioni e riferimenti agli studi del professore pugliese.

${ }^{152} \mathrm{Il}$ dibattito era stato aperto con l'articolo di R. Savelli, Alla ricerca di una formula. Che cosa vuole l'«Unità»?, «L'Unità», II, 7 marzo 1913, cui rispose G. Salvemini, Alla ricerca di una formula, «L'Unità», II, 7, 14 e 21 marzo 1913; le risposte di Salvemini sono riprodotte in Id., Opere IV, Il Mezzogiorno e la democrazia italiana, vol. I cit., pp. 292-303. Altri momenti del dibattito sono G. Salvemini, Postilla a G. Zagari, Polemiche sul metodo, «L'Unità», II, 25 aprile 1913, la postilla è riprodotta in G. Salvemini, Opere IV, Il Mezzogiorno e la democrazia italiana, vol. I cit., pp. 304-308, e G. Salvemini, Quel che l'Unità non può dare e quel che non vuol fare, «L'Unità», II, 2 maggio 1913, riprodotto in Id., Opere VIII cit., pp. 409-412.

${ }^{153}$ Vedi su questo C. Lacaita, Salvemini e il federalismo, in G. Pescosolido (a cura di), Gaetano Salvemini (1873-1957). Ancora un riferimento. Atti del convegno di studi tenutosi a Roma l'11 e il 12 dicembre 2007 in collaborazione con la Fondazione "Ernesto Rossi e Gaetano Salvemini”, Lacaita, Manduria-Bari-Roma, 2010, pp. 47-74. Cfr. inoltre F. Grassi Orsini, Salvemini e il federalismo, in M. Degl'Innocenti (a cura di), Gaetano Salvemini e le autonomie locali, Lacaita, Manduria-Bari-Roma, 2007, pp. 87-91, e I. Biagianti, Il federalismo di Salvemini, in G. Cingari (a cura di), Gaetano Salvemini tra politica e storia cit., pp. 198-226.

${ }^{154}$ Camillo da Lodi [C. Berneri], L'autodemocrazia, «Volontà», I, 1 giugno 1919, riprodotto in Id., Pietrogrado 1917 Barcellona 1937. Scritti scelti, a cura di Pier Carlo Masini e Alberto Sorti, La Fiaccola, Ragusa, 1990, pp. 30-34, in Id., Il federalismo libertario, a cura di Patrizio Mauti, La Fiaccola, Ragusa, 1992, pp. 9-12, e in Id., Scritti scelti, Zero in condotta, Milano, 2013, pp. 54-57.

${ }^{155}$ Vedi C. Strambi, L'inquieta attitudine: Camillo Berneri e la vicenda politica dell'anarchismo in Italia. Secondo Libro cit., pp. 84-85. 
Berneri torna a occuparsi di Rivoluzione Russa, avendo però trovato un nuovo e interessante aspetto da cui i partiti a carattere rivoluzionario dovrebbero, a suo parere, trarre spunto. Vale la pena soffermarsi già sul termine adoperato, quello di «autodemocrazia», termine inedito e quanto meno insolito nel vocabolario del giovane anarchico, termine che può essere considerato una rielaborazione personale delle idee di riforma e di rinnovamento della democrazia che Berneri può aver derivato dal confronto con Salvemini. L'autodemocrazia è, secondo il giovane anarchico, un'organizzazione politica democratica in cui sia possibile accrescere la partecipazione attiva e diretta del popolo al governo della res publica attraverso organi in cui studiare problemi, discutere soluzioni e praticare l'amministrazione. La Russia rivoluzionaria, sostiene Berneri, sta offrendo un esempio interessante da questo punto di vista, poiché i bolscevichi e il «regime dei Soviet» vi starebbero mettendo in atto una forma sperimentale di quella «democrazia integrale» teorizzata e indicata «nei disegni politico-filosofici dei principali pensatori della Francia rivoluzionaria e democratica» ${ }^{156}$. L'organismo del soviet è dunque «una derivazione dell'autonomia federalista ed è in antitesi con la tendenza accentratrice del socialismo di Stato» ${ }^{157}$, un organo di partecipazione e di autogestione da cui si può prendere esempio per controbilanciare - fino a rendere superflua - la presenza del centralismo statale ${ }^{158}$. Parlando di autonomia federalista Berneri pare agganciarsi per la prima volta nella sua produzione scritta all'idea della libera e spontanea federazione di associazioni o comuni autonomi che, secondo il pensiero anarchico, si sostituiranno al governo politico. Berneri tuttavia non supporta la sua tesi con i testi classici del federalismo anarchico o libertario, bensì a partire dalla fílosofia politica di Rousseau e soprattutto con riferimento ad altri autori democratici e socialisti che, nel dibattito della Francia del post 1848, si dissero favorevoli a forme di democrazia diretta e critici del parlamentarismo, della delega e della rappresentatività del potere come Rittinghausen, Considerant e Leverdays.

È lecito ritenere che la riflessione sul federalismo e sullo sviluppo della partecipazione popolare possa essere stata stimolata dall'influenza o dal confronto con Salvemini, da sempre interessato al tema del federalismo e del contrasto al centralismo statale. Il riferimento al dibattito francese durante la rivoluzione va ritenuto in particolare uno spunto quasi sicuramente tratto dal professore pugliese, già autore di uno studio sulla Francia rivoluzionaria del XVIII secolo ${ }^{159}$ e le cui

\footnotetext{
${ }^{156}$ Camillo da Lodi [C. Berneri], L'autodemocrazia cit.

${ }^{157}$ Ibid.

${ }^{158}$ Berneri parla dei soviet come organismi sperimentali interessanti di cui analizzare e immaginare le possibili applicazioni in senso rivoluzionario, egli non ha ovviamente idea - al pari di molti dei suoi compagni, come abbiamo visto - di come stia evolvendo l'esperienza sovietica in Russia. Vedi su questo G. Carrozza, Il "sovietismo" di Camillo Berneri, in Camillo Berneri, singolare/plurale. Atti della giornata di studi, Reggio Emilia, 28 maggio 2005, Edizioni Biblioteca Panizzi e Archivio Famiglia Berneri-Aurelio Chessa, Reggio Emilia, 2007, pp. 53-55.

${ }^{159}$ G. Salvemini, La rivoluzione francese (1788-1792), Pallestrini \& C., Milano, 1905; nel 1919 peraltro viene pubblicata a Roma la quarta edizione per i tipi de La Voce.
} 
osservazioni avrebbero potuto essere state sviluppate nel corso delle sue lezioni di Storia Moderna presso l'Istituto di Studi Superiori che il giovane anarchico frequenta già durante il suo primo anno accademico ${ }^{160}$. Anche se non è stato possibile trovare conferme certe negli archivi dell'Università degli Studi di Firenze ${ }^{161}$, riteniamo comunque che le idee di Rousseau riportate da Berneri nel suo articolo siano senza alcun dubbio derivate da letture o da suggestioni salveminiane, soprattutto considerando questo passaggio:

Qualcuno potrà osservare che il Rousseau repubblicano considera la «democrazia» come il governo in cui la massa del popolo gestisce direttamente gli affari comuni; il Rousseau liberal-moderato afferma che la democrazia si può adattare ai piccoli Stati [...]. Ma questa osservazione non ha valore critico, considerato che il federalismo è decentratore e autonomista per eccellenza.

Il governo, secondo il Rousseau, dev'essere il mandatario e l'esecutore della volontà generale e solo a questo patto è legittimo; «donde segue che i depositari del potere esecutivo non sono i padroni del popolo ma i suoi funzionari, che il popolo può istituirli e destituirli quando crede, che per essi non può essere questione di trattare col popolo ma di ubbidire» ${ }^{162}$.

La citazione riportata nella parte finale del passaggio è identica a quella utilizzata da Salvemini ne La rivoluzione francese, ne ricalca in pieno non solo i concetti, ma anche le parole:

Il governo dev'essere il mandatario e l'esecutore della volontà generale e solo a questo patto è legittimo: «donde segue che i depositari del potere esecutivo non sono i padroni del popolo ma i suoi funzionari, che il popolo può istituirli e destituirli quando crede, che per essi non può essere questione di trattare col popolo ma di obbedire ${ }^{163}$.

Lo scritto di Salvemini è senza dubbio una delle fonti dirette utilizzate da Berneri per scrivere questo articolo, persino l'interpretazione di Rousseau è

${ }^{160}$ Anno $1^{\circ}$, Studi, in Carriera Studente di Berneri, Luigi, p. 143, in ASUF, Regio Istituto di Studi Superiori, Pratici e di Perfezionamento in Firenze. Sezione di Filosofia e Filologia. Registro della carriera scolastica degli studenti X (1914-1917).

${ }^{161}$ Le ricerche da noi svolte sui manoscritti e sui materiali di lavoro presso l'Archivio Salvemini non hanno fatto emergere appunti di lezioni o altre evidenze in questo senso, allo stesso modo quelle svolte nell'Archivio Storico e nella Sala del Senato Accademico dell'Università degli Studi di Firenze. C'è da rilevare che comunque parte del repertorio relativo a quegli anni non risulta ad oggi accessibile per la consultazione e che comunque allora non si teneva precisa registrazione degli argomenti trattati durante i corsi - i registri delle lezioni iniziano a essere compilati e conservati a partire dal 1956, vedi F. Capetta e S. Piccolo (a cura di), Archivio storico dell'Università degli Studi di Firenze (1860-1960). Guida inventario, Firenze University Press, Firenze, 2004, p. 186.

${ }^{162}$ Camillo da Lodi [C. Berneri], L'autodemocrazia cit.

${ }^{163} \mathrm{G}$. Salvemini, La rivoluzione francese cit., p. 55. 
palesemente mutuata da quella fornita dal professore pugliese: anche quest'ultimo aveva opposto un Rousseau «socialista anarchico» a un Rousseau «liberale moderato» che ritiene che «il governo in cui la massa del popolo gestisce direttamente gli affari comuni, non si può adattare [...] che ai piccoli Stati, come quelli dell'antichità ${ }^{164}$. È d'altro canto necessario porre in rilievo come, nel suo studio, Salvemini abbia posto l'accento soprattutto su quest'ultimo aspetto del pensiero politico di Rousseau (favorevole al sistema rappresentativo, diffidente degli strati popolari, incline a una pratica di caute riforme rispetto a violente scosse rivoluzionarie, persino disposto a mettere da parte le rivendicazioni repubblicane di fronte a un sovrano o a un ministro disposto a intraprendere un'opera riformatrice) ${ }^{165}$, laddove Berneri fa invece emergere e concentra l'attenzione sul lato «socialista anarchico»» del filosofo ginevrino. In altre parole, è già possibile riconoscere un'elaborazione politica in certa misura autonoma da parte di Berneri, nonostante le suggestioni e le citazioni vengano chiaramente attinte da letture salveminiane.

Tornando all'articolo berneriano, ci sembra a questo punto altrettanto plausibile l'ipotesi che il dibattito tra i repubblicani francesi del 1848 vi compaia quanto meno su suggestione salveminiana, se non addirittura su suggerimento di lettura dello stesso, magari a seguito di curiosità espresse dallo studente anarchico in privato o al termine di una lezione; diversamente appare difficile comprendere come il giovane anarchico abbia potuto interessarsi a queste tematiche e come delle fonti politiche così insolite e del tutto inedite nei suoi articoli precedenti possano essere apparse in un suo scritto. Berneri cita anche il Proudhon de L'Idea generale della rivoluzione nel XIX secolo (1851), riportando un suo giudizio di sfiducia verso i rappresentanti eletti per suffragio universale o per acclamazione del popolo. Tuttavia il giovane anarchico non sembra considerare che, all'interno della stessa opera, Proudhon avesse duramente criticato tanto le teorie del Rittinghausen quanto, in generale, quelle dei fautori di una democrazia diretta ${ }^{166}$. Berneri appare tutto sommato poco attento alle diverse posizioni espresse dai vari autori citati nel suo articolo e alla natura del dibattito sulla partecipazione democratica nella Francia rivoluzionaria del $1848^{167}$, ma più interessato a porre in relazione queste idee politiche con l'«immenso campo sperimentale del socialismo» ${ }^{168}$ costituito dall'esperienza dei soviet russi: questi, secondo il giovane anarchico, potrebbero essere un organo attraverso cui

\footnotetext{
${ }^{164}$ Ivi, p. 56.

${ }^{165}$ Ivi, pp. 56-58. Nelle osservazioni conclusive Salvemini arriva a sostenere che, per quanto riguarda la prassi politica, «il Rousseau non era una vero e proprio rivoluzionario, e non senza ragione si è detto che, se fosse vissuto fino alla Rivoluzione, o sarebbe stato ghigliottinato anche lui, o sarebbe morto di crepacuore dopo pochi mesi, vedendo quale applicazione facessero i suoi discepoli delle sue teorie».

${ }^{166} \mathrm{Cfr}$. P.-J. Proudhon, Idée générale de la révolution au XIX $X^{e}$ sièclee, Garnier Frères, Paris, 1851 , tr. it. Centro editoriale toscano, Firenze, 2001, pp. 155-181.

${ }^{167}$ A tal proposito si veda S. Mastellone, Storia della democrazia in Europa. Dal XVIII al XX secolo, Utet, Torino, 2006, pp. 74-79.

${ }^{168}$ Camillo da Lodi [C. Berneri], L'autodemocrazia cit.
} 
permettere al popolo di partecipare direttamente alle decisioni politiche e all'amministrazione delle cose.

Il federalismo libertario inizia insomma ad essere affrontato dal giovane anarchico, anche se non in modo dottrinario: Berneri dimostra di essere più che altro consapevole della presenza di questa idea tanto all'interno del movimento anarchico quanto nella tradizione repubblicana e socialista, e dunque del suo essere un possibile punto di convergenza tra le forze e i movimenti rivoluzionari italiani. Inoltre ci sembra che appaia altresì evidente che il giovane anarchico non sia ancora giunto a studiare le teorie federaliste della propria corrente politica, poiché non lo vediamo ancora citare un solo autore e un solo testo classico dell'anarchismo: 1o stesso utilizzo di Proudhon, come si è visto, è volto soprattutto a sottolineare l'esistenza nella tradizione rivoluzionaria e democratica francese di pensatori che abbiano argomentato a sfiducia della delega e dei rappresentanti del popolo, non a dare ragione delle idee libertarie e della teoria federalista del socialista francese. Il pensiero anarchico, nella varietà e complessità dei suoi autori e delle sue teorie federaliste, non deve essere ancora presente nel giovane Berneri che, molto probabilmente, ne ha per il momento una conoscenza non certamente approfondita: non dimentichiamo che il suo passaggio all'anarchismo è avvenuto sul finire del 1915 e che inizialmente il suo attivismo si è rivolto soprattutto verso temi antimilitaristi, ma soprattutto che dal 1917 al 1919 la sua attività e le sue possibilità di studio devono essere state per forza di cose sporadiche ed episodiche, per via del servizio militare e dei periodi trascorsi al fronte. I suoi articoli del periodo confermano, tra l'altro, un maggiore interesse verso il presente e il commento dell'attualità piuttosto che una riflessione sui principi teorici, sui testi classici o sui "maestri”" dell'anarchismo.

L'idea che affascina e che risveglia l'interesse di Berneri è dunque, ci sembra, quella di trarre esempi e lezioni politiche rivoluzionarie dallo studio della storia. Nello specifico, egli arriva a ritenere che parte delle matrici politiche repubblicane del 1848 e l'esperimento dei soviet della Russia rivoluzionaria possano condividere una radice libertaria comune e che possano non solo dialogare ed essere messi in relazione tra loro, ma anche rappresentare un esempio o, perché no, una concreta soluzione di autogoverno e autogestione da opporre al centralismo statale e al principio di rappresentanza e di delega politica. Se anche può apparire irreale, secondo Berneri, «trovare rimedio ai tanti mali prodotti dall'attuale sistema» nelle teorie politiche di metà Ottocento, è però utile «prendere in considerazione il loro programma», e pertanto il giovane anarchico ritiene che

l'istituzione di clubs popolari, ove tutte le questioni sociali fossero liberamente e seriamente discusse, permetterebbe al popolo di divenire capace di prendere parte attiva, diretta e feconda agli affari della comunità, di poter esercitare un controllo rigoroso ed equo sul funzionamento degli organi sociali.

Tutti coloro che affermano il diritto del popolo di affermare la propria volontà riguardo ai sistemi di vita politica, devono studiare fra i problemi odierni quello dell'autodemocrazia, che potrebbe essere un buon obiettivo per le offensive dei 
partiti di avanguardia, che potranno dirigere i loro sforzi verso una meta comune: la emancipazione dei lavoratori dall'oligarchia demagogica ${ }^{169}$.

L'articolo L'autodemocrazia è in definitiva uno dei primi risultati tangibili e documentabili dell'influenza di Salvemini su Berneri e costituisce un primo tentativo di elaborare una personale riflessione politica da parte dello studente anarchico, che tenderà di qui in poi a portare entro la riflessione anarchica contenuti e idee a lei prossimi, come ad esempio la critica alle istituzioni parlamentari e in generale a qualunque potere costituito, espressa da diverse voci del dibattito filosofico politico del XIX secolo. L'articolo è anche il primo segnale di un'altra influenza salveminiana decisiva, vale a dire quella del concretismo: se è dunque possibile, sembra voler sostenere Berneri, rendere concreta e operativa la critica anarchica allo Stato e trovare un modello in grado di sostituire quello della rappresentanza, lo si può fare anche richiamandosi sia ai principi della democrazia diretta e partecipata esposti dai repubblicani francesi nel corso del '48, sia - nel frangente storico attuale - ai soviet russi, i nuovi organi sperimentali di partecipazione sulla base dei quali sta avvenendo una rivoluzione socialista. Non solo dunque idee e principi, ma anche e soprattutto proposte che abbiano dimostrato la loro applicabilità - come, per l'appunto, quella dei clubs popolari e dei soviet - e dunque la possibilità concreta di trasformare l'ordinamento esistente attraverso organi di discussione e democrazia diretta, in cui il popolo possa educarsi alla gestione del potere e all'amministrazione.

L'invito rivolto da Berneri ai «partiti di avanguardia» è dunque quello di approfondire lo studio di queste esperienze e di queste soluzioni, un invito che sembra includere non solo gli anarchici, ma anche le altre forze politiche con cui potrebbe essere possibile trovare una qualche forma di convergenza, come repubblicani, sindacalisti e socialisti recuperati - va da sé - a quella che il giovane anarchico ritiene dovrebbe essere la loro identità. La ricerca del blocco e dell'unione delle forze rivoluzionarie comincia in questi anni a comparire con maggiore insistenza e in questo caso, per la prima volta, viene legato allo studio e all'organizzazione di una battaglia specifica, quella dell'autodemocrazia o, meglio, dell'autonomismo e del federalismo.

\section{Concretismo rivoluzionario - lo studio dei problemi}

I fermenti rivoluzionari non hanno d'altronde abbandonato l'Italia, anzi gli squilibri sociali ed economici lasciati in eredità dalla Grande Guerra insieme all'esempio della Rivoluzione Russa stanno facendo da combustibile per nuove fiammate radicalmente sovversive. I movimenti, i partiti e i sindacati "di classe" conoscono nel dopoguerra una crescita sensibile, il movimento anarchico sta in particolare riprendendo progressivamente vivacità dopo la stretta repressiva subita durante il conflitto e tenta di darsi un indirizzo comune fondando, durante il suo secondo Congresso nazionale tenutosi peraltro a Firenze (12-14 aprile 1919), l'Unione comunista anarchica italiana (UCAI) che diverrà in seguito l'Unione Anarchica Italiana (UAI). La situazione nella penisola è incandescente e i moti

\footnotetext{
${ }^{169}$ Camillo da Lodi [C. Berneri], L'autodemocrazia cit.
} 
contro il caro-viveri che scoppiano tra primavera ed estate assumono carattere insurrezionale; gli anarchici intravedono la possibilità di indirizzare queste sommosse in senso rivoluzionario e sono promotori di un'unità dei partiti e dei movimenti rivoluzionari ${ }^{170}$. La prima maturazione delle idee politiche di Berneri si inscrive in questa concitata fase, che si prolungherà per un biennio - il Biennio Rosso, appunto ${ }^{171}$; come detto, intendiamo analizzare questa fase e quelle successive alla luce delle determinanti influenze salveminiane, di cui sinora non ci pare sia stato reso conto in modo sufficientemente adeguato e approfondito.

La questione dell'unione delle forze torna in questo periodo d'attualità e Berneri, che non ha mai smesso di credere nella necessità di un blocco sovversivo, riprende le proprie riflessioni in proposito nel corso del 1919. Il primo maggio esce sul settimanale «Il Libertario» un suo primo articolo sul tema, intitolato appunto $I$ problemi dell'unità rivoluzionaria ${ }^{172}$. Il titolo anche in questo caso ci sembra indicativo dell'influenza salveminiana sullo studente anarchico: riprendendo le suggestioni del concretismo, egli intende occuparsi di un singolo problema, l'unità rivoluzionaria, e di analizzarlo nei suoi diversi aspetti, cercando così di giungere a conclusioni e proposte operative che non derivino esclusivamente da principi generici e astratti; in altre parole, Berneri non intende semplicemente valutare quali siano i movimenti o i partiti che a livello di idee o formule possano sembrare convergere sulle posizioni degli anarchici nella creazione di un movimento rivoluzionario, ma intende rivolgere la sua attenzione agli indirizzi che vi vengono effettivamente affermati e che possano informarne l'azione. Egli afferma che

La rivoluzione è anzitutto e soprattutto la rottura violenta e collettiva della legalità da parte della classe soggetta. L'azione rivoluzionaria tende alla distruzione dello Stato poiché se non è certo che la funzione crei l'organo, è evidente che dall'organo dipende la maggiore o minore potenzialità della funzione $[\ldots]^{173}$.

Sul rovesciamento dello Stato le posizioni degli anarchici e dei socialisti di tendenza rivoluzionaria sarebbero dunque perfettamente coincidenti, tuttavia

\footnotetext{
${ }^{170} \mathrm{Si}$ veda a tal proposito F. Giulietti, Gli anarchici italiani dalla grande guerra al fascismo, Franco Angeli, Milano, 2015, pp. 85-144, e L. Di Lembo, Guerra di classe e lotta umana. L'anarchismo in Italia dal Biennio rosso alla Guerra di Spagna (1919-1939), Edizioni Biblioteca Franco Serantini, Pisa, 2001, pp. 25-86. Per un quadro generale si rimanda a R. Vivarelli, Storia delle origini del fascismo: l'Italia dalla grande guerra alla marcia su Roma, vol. I, Il Mulino, Bologna, 1991, pp. 500-520, e I. Barbadoro, Biennio rosso: lotte sociali e direzione socialista, in Id. (coordinata da), Storia della società italiana, vol. XXI, Teti, Milano, 1982, soprattutto pp. 231-266.

${ }^{171}$ Un'ampia ricostruzione generale di questo periodo è il già segnalato studio di C. Strambi, L'inquieta attitudine: Camillo Berneri e la vicenda politica dell'anarchismo in Italia. Secondo Libro cit

${ }^{172}$ Camillo da Lodi [C. Berneri], I problemi dell'unità rivoluzionaria, «Il Libertario», XVII, 1 maggio 1919.

${ }^{173}$ Ibid.
} 
secondo Berneri l'unità è ostacolata dal «dissenso riguardante la parte ricostruttiva della rivoluzione». È tuttavia necessaria, nonostante i dissensi, una valutazione di tipo tattico:

Credo che sia opportuno fondare l'unità rivoluzionaria sulla parte distruttiva della rivoluzione, lasciando al domani la lotta per la parte ricostruttiva.

[...] La rivoluzione attraverso le sue diverse fasi collauderà le diverse teorie sociali con l'attrito e col cozzo con la realtà e l'attuazione pratica ${ }^{174}$.

Data l'urgenza di sfruttare una crisi sociale che pare favorevole per avviare un movimento rivoluzionario, Berneri sostiene che è bene smettere

di porre ostacoli a quell'unità rivoluzionaria senza la quale credo impossibile l'insurrezione generale e se possibile credo si risolverebbe in un caotico rovesciamento di poteri e di sistemi politici ed economici, determinando crisi che metterebbero in grave pericolo le conquiste rivoluzionarie del proletariato ${ }^{175}$.

Si tratta, come si può vedere, di una prima personale rielaborazione del concretismo salveminiano da parte di Berneri: una posizione intransigente nell'accettare o meno certe alleanze potrebbe non solo arrestare il movimento insurrezionale, ma anche condurre a una serie di rivolte confuse e localizzate, incapaci sia di conseguire concreti obiettivi rivoluzionari generali sia di tutelare quanto i proletari sono riusciti a conquistarsi con le proprie lotte.

\section{Concretismo rivoluzionario - l'individuazione dei limiti}

Ancor più interessante è però l'altra faccia delle riflessioni berneriane sulle possibili alleanze in vista della rivoluzione. L'articolo I limiti dell'unità rivoluzionaria ${ }^{176}$, emerso dallo spoglio del settimanale «Il Libertario», mostra un ulteriore aspetto dello sviluppo di quello che potremmo definire il concretismo rivoluzionario di Berneri. Lo scritto, che non è mai stato censito dalle rassegne bibliografiche e che non è mai neanche comparso in nessuno studio o saggio sul Berneri, mostra come nell'elaborazione delle proprie posizioni egli abbia riconsiderato e riesaminato le speranze riposte nella rinascita di un nuovo blocco di forze insurrezionali, come accadde nel corso della Settimana Rossa. Se è vero, come lo studente anarchico aveva precedentemente sostenuto, che l'unità rivoluzionaria non deve essere vanificata da divergenze di principio, è altrettanto vero che esistono dei limiti ben precisi a questa unità. Se «si va determinando una tendenza unitaria sempre più spiccata che vorrebbe elaborare un programma unico estraendolo dall'immenso groviglio delle teoriche dominanti», ciò è giudicato da Berneri «naturale, ma irragionevole e utopista». Egli sostiene che

\footnotetext{
${ }^{174}$ Ibid.

${ }^{175}$ Ibid.

${ }^{176}$ Camillo da Lodi [C. Berneri], I limiti dell'unità rivoluzionaria, «Il Libertario», XVII, 15 maggio 1919.
} 
Errano coloro che vorrebbero pestare nel mortaio dell'unità rivoluzionaria tutti i cervelli pensanti ed innovatori per estrarne un succo unico che servisse d'ambrosia avveniristica all'umanità dolorante. Il pensiero è multiforme perché è in perenne formazione ed è illimitato poiché genera le idee la cui serie è illimitata.

[...] Fondere i "contrari" politici vorrebbe dire poggiare un'architettura su colonne barcollanti ${ }^{177}$.

Ideologie e sistemi possono apparire condivisi su più di un punto, ma ciò rischia di essere troppo generico e insufficiente per fondare una vera unità rivoluzionaria. Berneri giunge infine al vero bersaglio della sua polemica, vale a dire i repubblicani, e cita a questo proposito proprio quell'articolo di Leonetti apparso durante la guerra su «Il Grido» ${ }^{178}$, il periodico repubblicano cui aveva collaborato durante la guerra: secondo Berneri l'interpretazione leonettiana del sistema repubblicano come fondamento delle teorie socialista e anarchica è estremamente vaga, perché se ne dedurrebbe che «Repubblica e Anarchia non sono che etichette diverse incollate su una stessa bottiglia». L'articolo berneriano esemplifica in seguito la distanza tra repubblicani e anarchici:

Ogni partito ha il suo campo di pensiero e di azione: si può sconfinare nelle battaglie ideologiche, ma non si può abbattere le siepi e colmare i fossi ed arare con gli stessi buoi e coltivare con gli stessi semi, quando i terreni sono atti a diverse coltivazioni. [...] Senza urti e senza confusione di idee un'alleanza eterogenea è impossibile: è condizione necessaria di una solida alleanza politica una certa affinità nel modo di pensare e di volere il domani ${ }^{179}$.

Per Berneri è insomma possibile un'unità tra anarchici e socialisti rivoluzionari, ma difficile se non impossibile è coinvolgere in questo movimento anche i repubblicani, come avvenne nel 1914 durante la Settimana Rossa: anche se questi ultimi interpretassero il termine di repubblica come repubblica sociale, ciò non dissiperebbe la confusione, poiché allora anarchici e repubblicani

verrebbero a dimostrare che fra il significato letterale ed il valore reale della parola: Repubblica c'è una differenza sostanziale.

La Repubblica o è sociale o non è che una oligarchia borghese. Per un governo di dittatori alla Clemenceau né noi, né i socialisti ci batteremmo; per una Repubblica soviettista i repubblicani non metterebbero il naso fuori di casa ${ }^{180}$.

${ }^{177}$ Camillo da Lodi [C. Berneri], I limiti dell'unità rivoluzionaria cit. Il corsivo è originale.

${ }^{178}$ A. Leonetti, L'unicità del mezzo, «Il Grido», I, 5 dicembre 1917, vedi supra.

${ }^{179}$ Camillo da Lodi [C. Berneri], I limiti dell'unità rivoluzionaria cit. Si ricorderà che la formula «affratellamento, ma senza confusioni come senza urti, dei libertari tutti (repubblicani, socialisti, anarchici)» era tra i punti del programma dei repubblicani de «Il Grido»».

${ }^{180}$ Ibid. Il riferimento alla Rivoluzione Russa era allora una delle parole d'ordine scandite spesso e volentieri dai rivoluzionari, specialmente dai socialisti massimalisti, vedi $\mathrm{R}$. 
Riteniamo significativo porre in rilievo come Berneri richiami, ancora una volta, il soviet e l'esperienza russa, insomma quell'orizzonte di democrazia autogestita e di eguaglianza sociale che inizia a emergere tra i suoi interessi. Nella battaglia per il conseguimento di questi risultati non gli sembra possibile fare affidamento sui repubblicani, con ogni probabilità per via dei mancati sviluppi delle agitazioni promosse a suo tempo dal gruppo de «Il Grido». Lo studente anarchico, osservando un PRI sempre più distante da un impegno sociale e rivoluzionario, deve aver voluto tracciare una linea di confine netta: se con i socialisti rivoluzionari è possibile, oggi come durante le mobilitazioni anti-interventiste, fare un pezzo di strada, non altrettanto può dirsi per i repubblicani, che secondo lo studente anarchico non hanno più alcuna vocazione sovversiva.

Berneri è dunque a suo modo affamato di concretismo, ma in un senso diverso rispetto a quello di Salvemini: se quest'ultimo richiede lo studio dei problemi e l'elaborazione di soluzioni di riforma concrete da opporre ai sistemi e alle ideologie dei partiti, il primo si impegna invece nello studio delle questioni e nella ricerca di strategie rivoluzionarie realistiche e praticabili da opporre alle astrattezze. Così, parlando di unità rivoluzionaria, una piattaforma di vaste alleanze risulterebbe a suo avviso anche troppo vaga, poco solida, incapace insomma di incidere:

Tale fusione sarebbe fittizia e pregiudicherebbe l'azione del secondo periodo rivoluzionario: il ricostruttivo.

I blocchi eterogenei sono destinati a disgregarsi al contatto con la realizzazione dei principi.

Solo l'intesa fra gli elementi rivoluzionari socialisti è doverosa, è necessaria, è possibile ${ }^{181}$.

Non tarderà a giungere una risposta a Berneri e arriverà peraltro da una sua vecchia conoscenza, vale a dire da Dino Fienga, a suo tempo animatore dell'esperienza de «Il Grido». Rispondendo con una lettera su «Volontà» anche a un altro articolo berneriano, pubblicato sullo stesso giornale ${ }^{182}$, sul venir meno dello spirito rivoluzionario dei repubblicani, il militante repubblicano non nega che il suo partito abbia assunto un atteggiamento contraddittorio con quello tenuto dalla base, tuttavia ritiene

disonesto, speculando sulla ignoranza, far credere che tutti i repubblicani, tutti i generosi della Settimana Rossa, siano stati durante il periodo bellico pecore matte. Capisco e mi spiego la manovra dei dirigenti nostri che hanno fatto di tutto per farlo credere, $[\ldots]$ ma che gli estremisti concorrano a convalidare la menzogna francamente non me lo so spiegare o meglio me lo spiego benissimo quando sono elementi in mala fede ${ }^{183}$.

Vivarelli, Storia delle origini del fascismo cit., pp. 211-233.

${ }^{181}$ Camillo da Lodi [C. Berneri], I limiti dell'unità rivoluzionaria cit.

${ }^{182} \mathrm{Id}$., Il partito repubblicano di fronte alla rivoluzione, «Volontà», I, 1 maggio 1919.

${ }^{183}$ I repubblicani e noi, «Volontà», I, 16 giugno 1919. 
Fienga fa riferimento al proprio giornale e alle critiche rivolte alla dirigenza del PRI e ricorda che nelle fila repubblicane non mancano militanti che cercano di spingere il partito «più decisamente ancora a sinistra». Alla sua lettera fa seguito una nota redazionale - attribuibile con ogni probabilità a Luigi Fabbri, direttore della rivista - che, diversamente dalle posizioni di Berneri, si dimostra aperta verso i repubblicani in quanto «coloro che sono contro le istituzioni attuali, qualunque sia il partito cui appartengono, ci sono molto più vicini di quel che sembra». L'apertura è rivolta soprattutto alla base del PRI:

Per esempio, spesso quando ci siamo trovati a discutere coi repubblicani operai ed esponevamo loro il nostro programma, essi ci rispondevano che "quella era la loro repubblica". [...] Anzi avveniva questo, che i loro capi e dirigenti andavano d'accordo coi socialisti riformisti della Confederazione del Lavoro, mentre i gregari venivano più volentieri d'accordo con gli anarchici e con i sindacalisti. Inoltre il vivo spirito antimonarchico, rivoluzionario e insurrezionale degli elementi operai repubblicani in alcune località ci avvicinavano a loro anche più che ai socialisti, benché più di questi ci fossero lontani nelle idee ${ }^{184}$.

La postilla prosegue insistendo sulle affinità storicamente riscontrabili tra movimento repubblicano e movimento anarchico fino alla Settimana Rossa. A differenza della posizione berneriana, la nota redazionale afferma che la base del PRI possa essere coinvolta nel movimento rivoluzionario, purché faccia ritorno alle proprie origini:

L'antica formula mazziniana "libertà e associazione" trova una assai più larga esplicazione nel nostro programma anarchico e comunista, che ebbe un primo teorico in Pisacane vissuto e morto repubblicanamente e un assertore dal punto di vista filosofico nel pensatore repubblicano Giovanni Bovio ${ }^{185}$.

Pertanto, agli occhi di Fabbri, i repubblicani degni di questo nome dovrebbero tornare almeno a desiderare la rivoluzione, «il mezzo senza di cui non sarà possibile l'anarchia, ma neppure la repubblica!»

Il dibattito e il confronto tra anarchici e frange di sinistra del movimento repubblicano in questo periodo meriterebbe una trattazione a parte che ancora nessuno studio ha sufficientemente affrontato: nel nostro caso è interessante notare come, nelle battute di questo confronto, il gruppo de «Il Grido» rivendicasse il suo spirito rivoluzionario e come, dall'altra parte, il movimento anarchico non fosse del tutto ostile ad aprire un dialogo con almeno parte della base del PRI.

\section{Concretismo rivoluzionario - riflessione e autocritica}

\footnotetext{
${ }^{184}$ I repubblicani e noi cit.

${ }^{185}$ Ibid.
} 
Berneri tornerà in seguito a rivolgersi ad alcune anime del movimento repubblicano, ma per il momento non prende parte né cerca di sviluppare ulteriormente il dibattito, anche perché l'azione chiama: lo sciopero generale del 2021 luglio 1919 si avvicina e gli anarchici sono decisi a dargli uno sbocco rivoluzionario. Lo studente fa giusto in tempo a preparare e a dare i suoi primi due esami universitari ${ }^{186}$ prima di prendere parte allo sciopero. Tuttavia la sperata rivoluzione, complici anche gli appelli del PSI e della Confederazione Generale del Lavoro, non avverrà; Berneri verrà per giunta arrestato nel corso di una manifestazione presso la Casa del Popolo di Sestri Ponente e, risultando ancora arruolato, verrà confinato all'isola di Pianosa fino al termine del servizio militare.

Ciò non impedirà al lodigiano di riflettere sul fallimento degli scioperi e di esprimere i suoi dubbi sull'unità coi socialisti a distanza di circa un anno, quando pubblicherà l'articolo Il momento attuale e l'unità rivoluzionaria sul quotidiano anarchico appena fondato «Umanità Nova» ${ }^{187}$. Pur continuando a ritenere che anarchici e socialisti siano accomunati «più dalle contingenze attuali che dall'azione futura» e che sia comunque più che mai necessario stabilire le condizioni di un'unità rivoluzionaria, Berneri non può non osservare il divario che separa i fermenti della base dei militanti e l'azione parlamentare dei deputati di tendenza rivoluzionaria, pertanto afferma che

Due sono le posizioni che i deputati massimalisti possono assumere di fronte al precipitare degli avvenimenti: o pensano impossibile la rivoluzione per difficoltà insolubili [...]; o pensano la rivoluzione necessaria e possibile ed allora è loro dovere, visto che il parlamento non è organo di conquista rivoluzionaria, d'abbandonarlo e svolgere una attiva e seria opera di propaganda e di organizzazione rivoluzionaria in seno alle masse ${ }^{188}$.

E più avanti insiste:

Anche ultimamente [...] abbiamo visto la direzione del P.S. e l'Avanti! stare alla finestra, ad attendere, ad osservare, mostrando di non essere all'altezza della situazione, della possibilità cioè che gli scioperi [...] sboccassero in un'azione oltrepassante gli scopi economici immediati. [...]

Nei periodi di calma i tribuni massimalisti arringano le folle con la loro violenza verbale e parlano della rivoluzione come d'un fatto imminente e necessario; nei periodi di bufera corrono ai ripari o si mantengono in quelle posizioni di attesa, di incertezza, di riserva, che non sono certamente all'altezza delle necessità del momento ${ }^{189}$.

${ }^{186}$ Dopo aver frequentato dei corsi integrativi, il 18 luglio Berneri dà gli esami di Storia Moderna con Salvemini (19/30) e di Pedagogia con Calò (26/30), vedi Anno 2 , Esami, in Carriera Studente di Berneri, Luigi cit.

${ }^{187}$ Camillo da Lodi [C. Berneri], Il momento attuale e l'unità rivoluzionaria, "Umanità Nova», I, 9 marzo 1920.

${ }^{188}$ Ibid.

${ }^{189}$ Ibid. 
Riemergono, giova sottolinearlo, le antiche fratture che avevano portato Berneri fuori dal PSI e che egli aveva cercato di declinare nella produzione scritta immediatamente successiva: se alle parole non fanno seguito fatti conseguenti, non può esservi la concreta possibilità di un'unità rivoluzionaria tra anarchici e socialisti e lo studente anarchico ha fame di concretezza almeno quanto ne ha di rivoluzione. Le nuove esigenze si intrecciano alle precedenti.

\section{Problemismo e rivoluzione}

Nel periodo del confino a Pianosa la collaborazione di Berneri alla stampa e al movimento anarchico si fa più rarefatta, ma uno dei suoi contributi riporta alla luce quell'ex giovane socialista cresciuto e formatosi sotto l'influenza del culturismo, quel Berneri che crede nella necessità di un'elevazione culturale del proletariato e che intende legare questa necessità al momento rivoluzionario che attraversa la penisola. Pubblicato su «Volontà», l'articolo si occupa della recente conquista della giornata lavorativa di otto ore e di come valorizzare il tempo libero ottenuto per migliorare la cultura degli operai ${ }^{190}$. Berneri appare molto preoccupato che le ore di riposo e di svago possano svolgersi nei «consueti dannosi ritrovi, osterie e postriboli in ispecie» e auspica per contro che il popolo si rivolga a uno studio serio e approfondito che ne migliori la cultura e ne aiuti l'emancipazione sia umana che sociale:

Assorto in diverse, suggestive e piacevoli e nuove occupazioni dello spirito l'operaio dimenticherà, senza grandi sforzi di volontà e senza dannose ricadute, le vecchie consuetudini plebee $[\ldots]$.

Non credo che la cultura popolare sia un diversivo della lotta di classe [...]. Credo che l'operaio formandosi una cultura meno caotica e più profonda sentirà maggiormente il bisogno di redimersi, vedrà in tutta la sua asprezza il contrasto fra la sua ascesa ed il peso morto delle condizioni ambientali, vivrà la lotta di classe con idee più lucide, con intenti più vasti ed umani, sarà meno violento e più forte $[\ldots]^{191}$.

L'articolo risulta interessante non solo perché mostra in parte un nuovo emergere dello spirito culturista cui Berneri aveva aderito al tempo della militanza nel movimento giovanile socialista, ma anche perché questo aspetto appare strettamente collegato allo sviluppo di un nuovo indirizzo di provenienza chiaramente salveminiana, ossia quello dello studio dei problemi e della fuga dalle soluzioni semplicistiche. Lo studente anarchico, come abbiamo visto, sta facendo suo questo indirizzo, declinandolo in un'ottica rivoluzionaria; nel caso specifico, ritiene che lo studio e la preparazione culturale siano «condizione necessaria, benché non sufficiente, di una Rivoluzione che esca dall'orbita dei soli tumulti sporadici o dei colpi di stato ${ }^{192}$. La cultura popolare, secondo Berneri, si rende dunque necessaria

\footnotetext{
${ }^{190}$ Camillo da Lodi [C. Berneri], I doveri di una conquista classista. Le 8 ore di lavoro e la cultura popolare, «Volontà», I, 16 settembre 1919.

${ }^{191}$ Ibid.

${ }^{192}$ Ibid.
} 
soprattutto in relazione al momento di crisi sociale che l'Italia sta attraversando, un momento che potrebbe aprire a uno sbocco rivoluzionario che ancora però non si è realizzato, anche perché il proletariato risulta scarsamente preparato e finisce pertanto col rimanere succube o agli appelli di normalizzazione - che fanno sì che $\mathrm{i}$ tumulti restino episodi sporadici e slegati tra loro - o al mito del colpo di mano con cui si rischia di sostituire alla vecchia autorità una nuova autorità, vanificando così lo sforzo libertario. In definitiva, se senza approfondimento e studio dei problemi e senza elaborazione di obiettivi e definizione di proposte concrete e praticabili non si possono fare le riforme, figurarsi una rivoluzione. La preoccupazione di una maggiore preparazione militante come via per fuggire dalle formule vaghe e astratte dell'ideologia emergerà con maggiore chiarezza successivamente, quando i contributi berneriani torneranno a essere continui, a partire dal rientro dal confino terminato all'inizio del novembre dello stesso anno.

Esemplare da questo punto di vista è la serie di articoli sulla Rivoluzione Russa pubblicata su «Volontà» a partire dal dicembre del 1919 e significativamente intitolata I problemi della Rivoluzione, altro riferimento alla linea concretista mutuata da Salvemini e applicata all'ambito delle idee rivoluzionarie. Il contenuto di questa serie e le opinioni di Berneri intorno alle questioni che affronta (l'approvvigionamento delle città, dell'integrazione dei ceti medi nel processo rivoluzionario, delle abitazioni e della possibile nascita di nuove disuguaglianze economiche e sociali) sono state illustrate da Carrozza ${ }^{193}$; noi qui proponiamo un'ulteriore interpretazione di questi articoli, individuandovi non solo una conferma della profondità dell'influenza salveminiana sullo studente anarchico ma anche il parallelo inizio dell'approfondimento da parte di quest'ultimo del pensiero anarchico, in particolare di quello di Kropotkin. Gli articoli pongono spesso in relazione i problemi della Rivoluzione Russa con le storiche esperienze rivoluzionarie francesi il cui studio, come abbiamo visto, è nato senza alcun dubbio sotto l'influenza di Salvemini e a partire dal suo libro La rivoluzione francese. A questa fonte si è adesso affiancata anche quella dei testi di Kropotkin che trattano gli stessi temi, in particolare La grande Rivoluzione (1909) e La conquista del pane (1892). Nessuno studio o saggio critico finora pubblicato ha mai colto l'influsso salveminiano su questa produzione berneriana ${ }^{194}$, che a noi invece appare evidente anche nel modo in cui Berneri cita e utilizza alcune fonti classiche del pensiero anarchico per cercare di leggere e di interpretare alcune delle principali questioni che si stanno presentando ai rivoluzionari russi, insistendo spesso e volentieri sulla necessità dello studio profondo dei problemi in modo da elaborare soluzioni "salveminianamente"

${ }^{193}$ G. Carrozza, En Russie bolscheviste. L'analisi di Berneri sull'Unione Sovietica all'inizio degli anni '20, in G. Berti, G. Sacchetti (a cura di), Un libertario in Europa cit., soprattutto le pp. 57-58.

${ }^{194} \mathrm{Nel}$ suo recente studio, Strambi parla in termini generici dell'approccio problemistico presente in questi articoli, ma associandolo all'influenza di Kropotkin. Cfr. C. Strambi, L'inquieta attitudine: Camillo Berneri e la vicenda politica dell'anarchismo in Italia. Secondo Libro cit., pp. 100-105. 
concrete e praticabili, che aiutino lo sviluppo della rivoluzione nella sua fase ricostruttiva.

Così, ad esempio, Berneri si esprime sul rapporto tra città e campagna:

Il problema dei rapporti tra centri urbani e le campagne in periodo rivoluzionario, o post-rivoluzionario, rispetto alla fase insurrezionale della rivoluzione è un problema complesso; ed io creda sia necessario avere il senso della complessità di tali problemi vitali se non si vuole rimanere appagati da soluzioni sempliciste e illusorie, soluzioni che pregiudicano l'azione rivoluzionaria di domani ${ }^{195}$.

Più avanti nello stesso articolo, Berneri insiste sull'argomento affermando che questa operazione va fatta

senza la pretesa di fare del revisionismo ipercritico ma con la convinzione della necessità di offrire ai nuovi problemi postici innanzi dalla realtà rivoluzionaria nuove soluzioni e la piena coscienza del dovere di liberare il comunismo dagli ottimismi semplicisti che arrestano il suo sviluppo teorico e minacciano gravemente le sue pratiche applicazioni ${ }^{196}$.

$\mathrm{Si}$ vede bene da queste citazioni quanto il problemismo e il concretismo salveminiani stiano esercitando influenza sullo studente anarchico, spingendolo ad affermare che la rivoluzione non si fa con in mano i programmi di massima e la purezza dell'ideale, bensì confrontando e misurando le proprie idee coi fatti concreti.

La tendenza ritorna molto spesso anche in altre occasioni:

Questa serie di articoli si propone due scopi principali: $\mathrm{I}^{\circ}$ risolvere e modificare, nei dettagli, s'intende, il nostro programma politico ed economico, rispetto alle necessità strategiche della rivoluzione e alle ricostruzioni sociali che ad essa seguiranno; II $^{\circ}$ indirizzare il pensiero dei compagni ad una visione più ampia ed acuta delle nuove situazioni in cui il pensiero e l'azione anarchica si verranno a trovare di fronte alla rivoluzione ${ }^{197}$.

\section{Prosegue Berneri:}

L'anarchismo si trova di fronte al socialismo autoritario ed accentratore, che ha un piano programmatico che potrà essere aprioristico, ma che è attuale e organico nel suo complesso e ben delineato nei suoi dettagli, e deve quindi svolgere e diffondere il suo programma sì che l'azione popolare ne diventi la diretta espressione, il corollario realizzatore ${ }^{198}$.

\footnotetext{
${ }^{195}$ Camillo da Lodi [C. Berneri], I problemi della Rivoluzione. I rapporti fra la Città e le Campagne, «Volontà», I, 1 dicembre 1919, il corsivo è nostro.

${ }^{196} \mathrm{Ibid}$. Il corsivo è originale.

${ }^{197}$ Camillo da Lodi [C. Berneri], I problemi della rivoluzione. Parentesi, «Volontà», II, 16 gennaio 1920.

${ }^{198}$ Camillo da Lodi [C. Berneri], I problemi della rivoluzione. Parentesi cit.
} 
Il movimento anarchico rischia di giocare un ruolo subalterno nella rivoluzione per via dei suoi eccessivi pregiudizi di principio e della sua conseguente scarsa disposizione a studiare soluzioni concrete e programmi praticabili. Berneri rincara dunque la dose:

Uno dei più funesti errori è di rimanere abbracciati ai cadaveri dei Maestri, anche se di giganti come Bakounin, di preferire rivolgersi agli assertori di ieri dell'idea rivoluzionaria anziché procedere verso i richiami delle ulteriori realizzazioni di domani. [...] Io credo che per liberarci definitivamente da quella schiavitù dello spirito $[\ldots]$ occorra trattare su tutti i nostri giornali, rassegne o riviste, i problemi della rivoluzione, e trattarli sì da tendere a rispondere ad un bisogno profondamente sentito da molti compagni: quello che la stampa nostra sia una palestra di discussioni pratiche, un campo di studi e ricerche intorno ai problemi della vita sociale nel periodo rivoluzionario e post-rivoluzionario ${ }^{199}$.

Oltre a un'altra vasta serie di riferimenti salveminiani - l'insistenza continua sullo studio dei problemi, sulla necessità di indirizzare le discussioni sul concreto e di creare così un'arena di approfondimenti, elaborazioni, scambi e confronti sul come tradurre in pratica un programma (di ispirazione anarchica, nel caso specifico) - Berneri fa probabilmente qui per la prima volta cenno alla necessità per l'anarchismo di andare oltre un pensiero ancorato ai suoi "maestri", poco flessibile e poco disposto a rinnovarsi a confronto con la realtà: diventa necessario superare una «propaganda parolaia, vuota, fatta di sonorità superficiali, di audacia esteriore, sterile di risultati duraturi» e passare a una «che sia un complesso di soluzioni, relative e approssimative, dei grandi problemi sociali d'oggi e di domani, soluzioni conciliate e verificate, in un tutto armonico e compatto, nella nostra ideologia libertaria $\rangle^{200}$. Il ripetersi degli appelli allo studio dei problemi e dell'ancoraggio dell'ideologia alla realtà insieme al rifiuto dell'arroccarsi dell'anarchismo sui propri assoluti teorici e su posizioni pregiudiziali dimostrano, ancora una volta, a nostro parere, la forte influenza esercitata su Berneri dalla lezione critica di Salvemini.

La tendenza a tenere conto della complessità delle questioni sociali che impediscono all'anarchia di essere un progetto concretamente e immediatamente trasferibile dalla teoria alla prassi deriva senza dubbio direttamente più dal concretismo salveminiano - il cui influsso appare palpabile nelle pagine berneriane che abbiamo riportato - che non dal generale clima di crisi del positivismo di inizio Novecento a cui alcuni studiosi hanno fatto fin troppo ampio riferimento come influenza capace di spiegare la svolta dell'anarchico lodigiano ${ }^{201}$.

${ }^{199}$ Ibid. Il corsivo è originale.

${ }^{200}$ Ibid.

${ }^{201}$ Vedi a tal proposito soprattutto P. Adamo, Per un fondazione epistemologica dell'anarchismo: Camillo Berneri e l'empiriocriticismo, in Camillo Berneri, singolare/plurale cit., pp. 105-108, e Id., Introduzione a C. Berneri, Anarchia e società aperta. Scritti editi e inediti, a cura di Pietro Adamo, M\&B Publishing, Milano, 2001, 


\section{Il federalismo libertario per rinnovare l'anarchismo}

Durante e successivamente alla sua formazione universitaria, Berneri intensifica le collaborazioni e le pubblicazioni con la stampa anarchica. In particolare si possono ricordare quelle che abbiamo già scorso con «Volontà», rivista diretta da Luigi Fabbri, «Guerra di Classe», organo ufficiale dell'Unione Sindacale Italiana (USI), e quella con «Umanità Nova», quotidiano fondato e diretto da Errico Malatesta nel febbraio $1920^{202}$, il settimanale «Fede!» sorto nel settembre 1923 e diretto da Luigi Damiani, e il quindicinale «Pensiero e Volontà» fondato da Malatesta all'alba del 1924. Tra i diversi articoli che scrisse in questo periodo, iniziano a prendere corpo anche quelli di più compiuta riflessione sul federalismo libertario.

\section{Appunti sul federalismo}

Tra le carte dell'Archivio Famiglia Berneri-Aurelio Chessa si trova un manoscritto dal titolo $\mathrm{Il}$ valore civile del federalismo ${ }^{203}$ (il titolo è stato cancellato nell'originale) che può essere ritenuto una prima interessante articolazione della prima fase delle riflessioni berneriane. La moglie Giovanna Caleffi, prima responsabile delle carte di Berneri, ha invero collocato lo scritto nella seconda metà degli anni '20 e Pietro Adamo ha accettato questa datazione collegandolo alle letture di questi anni ${ }^{204}$, ma riteniamo che dovrebbe essere presa in esame un'ipotesi di retrodatazione del documento: nel periodo compreso tra il 1919 e i primi anni '20

soprattutto le pp. 15-25. C'è da dire che le idee di Salvemini e della filosofia empiriocriticista, pur ignorandosi a vicenda, presentano più di una prossimità, cfr. a tal proposito G. Pecora, $L a$ scuola laica. Gaetano Salvemini contro i clericali, Donzelli, Roma, 2015, pp. 25-31.

${ }^{202}$ Unico quotidiano che il movimento anarchico italiano sia mai stato in grado di produrre, «Umanità Nova» è una ricca testimonianza della forza, della presenza e della capacità organizzativa degli anarchici in questo periodo. Sulla sua storia vedi F. Schirone (a cura di), Cronache anarchiche. Il giornale Umanità Nova nell'Italia del Novecento (1920-1945), Zero in Condotta, Milano, 2010, specialmente le pp. 17-117, corrispondenti ai saggi di L. Di Lembo, Errico Malatesta e la nascita di «Umanità Nova», M. De Agostini, «Umanità Nova» quotidiano a Milano, febbraio 1920-marzo 1921. Un tentativo di spoglio, e G. Scaliati, "Umanità Nova» a Roma, maggio 1921-dicembre 1922.

${ }^{203}$ C. Berneri, "Il valore civile del federalismo", in AFB, fondo C. Berneri, cass. XI, ${ }^{\circ} 2$, Scritti di critica storica, letteraria, filosofica. Il manoscritto è riprodotto in Id., Anarchia e società aperta cit., pp. 133-135.

${ }^{204} \mathrm{La}$ datazione è stata proposta da Adamo nella nota introduttiva al testo pubblicato nell'antologia da lui curata (vedi Anarchia e società aperta cit., p. 133); su tale datazione ha concordato anche lo storico De Maria, cfr. C. De Maria, Camillo Berneri. Tra anarchismo e liberalismo, Franco Angeli, Milano, 2004, p. 131. 
Berneri avrebbe già potuto ricevere le influenze salveminiane ed essersi approcciato alle letture liberali e repubblicane ${ }^{205}$ sulla base delle quali Adamo ha invece proposto l'attuale datazione. Inoltre vi troviamo dei passi - tratti anch'essi da Salvemini - che compaiono anche in un altro suo articolo, Stato e burocrazia $(1920)^{206}$, che potrebbe dunque essere di poco anteriore o posteriore.

Il frammento berneriano esordisce con una dichiarazione di principio: «Il sistema federale ha un valore sociale, educativo, oltre che politico-economico» ${ }^{207}$, ma l'elemento sorprendente risiede nel fatto che l'anarchico citi a sostegno della sua tesi passaggi tratti da La democrazia in America (1835-40) del celebre pensatore liberale Alexis de Tocqueville, altro elemento che va a sostegno della nostra ipotesi di retrodatazione del manoscritto berneriano: l'anarchico lodigiano conosceva certamente il classico di Tocqueville prima del 1926, come dimostra una sua recensione all'edizione italiana de L'Antico regime e la Rivoluzione pubblicata su «Pensiero e Volontà» il 1 marzo 1926; in questa recensione, fino a oggi non adeguatamente considerata da studiosi e ricercatori, emerge chiaramente quanto da noi sostenuto, in quanto Berneri segnala tra l'altro che il pensatore francese sia «autore di quella Democrazia in America che sarebbe così utile avesse un'edizione italiana ${ }^{208}{ }^{2}$, una testimonianza chiara della sua conoscenza del testo e del suo contenuto, tanto da lamentarne l'assenza di una traduzione fruibile al pubblico italiano.

Tornando al contenuto del manoscritto, accanto a Tocqueville compaiono inoltre anche citazioni da Les istitutions politiques, judiciaires et administratives de l'Angleterre (1863) del meno noto studioso di diritto amministrativo Amable Charles de Franqueville ${ }^{209}$; queste affermano che il decentramento

«non consiste solo nell'evitare di riunire nelle mani del potere centrale, per affidarli ad amministrazioni locali, alcuni rami di pubblico servizio», ma «nel restringere, per quanto è possibile, l'intervento medesimo di pubbliche autorità, e nel lasciare ai cittadini l'amministrazione personale dei loro interessi comuni $\rangle^{210}$.

\footnotetext{
${ }^{205}$ In questo caso pare emergere in Berneri, oltre all'influenza della lezione federalista e concretista di Salvemini, anche il recupero da parte di quest'ultimo dei valori liberali e democratici all'interno di una prospettiva socialista. Vedi su questo punto G. Pecora, Socialismo come libertà. La storia lunga di Gaetano Salvemini, Donzelli, Roma, 2012, specialmente le pp. 59-84.

${ }^{206}$ C. B. [C. Berneri], Stato e burocrazia, «Umanità Nova», I, 25 dicembre 1920, riprodotto in C. Berneri, Il federalismo libertario cit., pp. 13-19.

${ }^{207}$ Camillo Berneri, "Il valore civile del federalismo" cit., c. 1.

${ }^{208}$ C. B. [C. Berneri], I libri, «Pensiero e Volontà», III, 1 marzo 1926.

${ }^{209}$ Amable Charles Franquet de Franqueville $(1840$ - 1919) fu uomo politico francese, membro del Consiglio di Stato dal 1860 al 1876, conosciuto soprattutto per i suoi studi sulle istituzioni inglesi tra cui, oltre alla già citata Les institutions politiques, judiciaires et administratives de l'Angleterre (1863), vi sono Du régime des travaux publics en Angleterre (1875), Le gouvernement et le parlement britanniques (1887), Le système judiciaire de l'Angleterre (1895).
} 
Comune e Associazione sono dunque come due scuole entro cui il cittadino si educa alla vita pubblica, allontanandosi dall'egoismo personale e familiare e imparando a operare secondo gli interessi generali. Berneri cita a questo proposito Jules Simon ${ }^{211}$ per sostenere che, nell'autonomia comunale, «'i cittadini avranno il sentimento della loro importanza e della loro dignità, il sentimento del loro diritto' e 's'ingrandiranno nella propria stima, mescolandosi nei pubblici affari'» ${ }^{212}$. Immediatamente dopo viene citato invece il maestro Salvemini, definito «federalista contemporaneo», che sostiene allo stesso modo che il sistema federale sia come una scuola di autoeducazione in quanto è il cittadino

che amministra sé stesso, si avvezza a contare solo sulla propria iniziativa e non su quella di un'autorità lontana; e nello stesso tempo che si sviluppa in lui il sentimento della propria individualità, si avvede che egli non è un atomo avulso da altri atomi e unito con un punto centrale, ma fa parte di un sistema molto più complesso, nel quale egli è strettamente solidale col suo vicino, e poi cogli altri meno vicini, e poi cogli altri più lontani; il sentimento dell'autonomia individuale si feconderà quindi in $\underline{\text { lui col sentimento della solidarietà sociale }}{ }^{213}$.

Nel suo manoscritto Berneri accompagna a questa citazione una nota con cui rimanda a un opuscolo «quasi introvabile» di Salvemini. Fino ad oggi nessuno studio è risalito alla fonte originale adottata dallo studente anarchico, persino Pietro Adamo, ripubblicando il testo del manoscritto, ha riprodotto integralmente la nota berneriana senza però fornire ulteriori specificazioni. Dalla nostra ricerca è invece emerso che la citazione è tratta, praticamente parola per parola, da un articolo di Salvemini, La questione di Napoli, apparso nel dicembre 1900 sul periodico socialista «Critica Sociale» e ripubblicato l'anno seguente in forma di opuscolo per i tipi della rivista ${ }^{214}$. Ecco le parole dello scritto salveminiano:

Nel sistema federativo il cittadino si educa alla vita pubblica, è lui che amministra sé stesso, si avvezza a contare sulla sua propria iniziativa e non su quella di un'autorità

${ }^{210} \mathrm{C}$. Berneri, "Il valore civile del federalismo" cit., c. 1. La sottolineatura è originale.

${ }^{211}$ Jules Simon (1814 - 1896) fu filosofo e uomo politico francese. Professore di Filosofia all'École normale e alla Sorbona, entrò nella vita politica quale repubblicano moderato nel 1848. Costretto a ritirarsi dopo l'ascesa di Napoleone III, si dedicò a studi politici e filosofici. Rientrato in politica nel 1871, divenne Primo Ministro dal 1876 al 1877 . Tra le sue opere Le devoir (1854) e La liberté politique (1871) potrebbero essere - in mancanza di rimandi chiari nel testo - quelle da cui Berneri avrebbe tratto la citazione.

${ }^{212} \mathrm{C}$. Berneri, "Il valore civile del federalismo" cit., cc. 1-2.

${ }^{213}$ Ivi, c. 2. Le sottolineature sono originali nel manoscritto.

${ }^{214}$ Rerum Scriptor [G. Salvemini], La questione di Napoli, «Critica Sociale», X, 1 e 16 dicembre 1900 e Id., La questione di Napoli (Come si sgominerebbero le camorre amministrative), Edizioni Critica Sociale, Milano, 1901. L'articolo è riprodotto in G. Salvemini, Opere IV, Il Mezzogiorno e la democrazia italiana, vol. II, Movimento socialista e questione meridionale, Feltrinelli, Milano, 1963, pp. 214-217. 
lontana; e nello stesso tempo che si sviluppa in lui il sentimento della propria individualità, si avvede che egli non è un atomo avulso dagli altri atomi e unito con un filo a somiglianza degli altri atomi con un punto centrale, ma fa parte di un sistema molto più complesso, nel quale egli è strettamente solidale col suo vicino, e poi cogli altri meno vicini, e poi cogli altri più lontani: il sentimento dell'autonomia individuale si feconderà quindi in lui col sentimento della solidarietà sociale ${ }^{215}$.

Seguendo il filo dell'argomentazione berneriana, l'idea per cui la partecipazione diretta contribuisca a sviluppare e a migliorare le capacità civili dei singoli appare insomma sostenuta da voci autorevoli, eppure per Berneri in ben pochi sono disposti a credere nella possibilità di decentrare il sistema sino a un autonomismo che ponga il cittadino nella condizione di amministrare sé stesso: si è portati a credere che l'accentramento sia una conseguenza inevitabile dello sviluppo urbano, pertanto il decentramento non sarebbe applicabile che ai più piccoli paesi. Per Berneri invece il decentramento ha basi egualmente solide tanto nelle metropoli quanto nel piccolo paese e a sostegno di questa tesi riprende a citare ampiamente proprio Salvemini: «Ne è esempio Londra, che benché molto più grande di Napoli, non è colpita dalle malattie burocratico-camorriste della città partenopea, perché amministrata federativamente $»^{216}$.

Il lodigiano passa quindi a supporre la possibilità di poter organizzare una grande città su basi federali:

In questo caso non formerebbe un unico Comune, ma dieci, venti Comuni, a seconda le sue condizioni topografiche e la configurazione degli interessi locali. In ciascuno di questi Comuni l'amministrazione non s'accentra in un unico consiglio, ma si scinde in parecchi consigli, ciascuno dei quali ha una propria amministrazione e un ben circoscritto compito da eseguire: istruzione, illuminazione, viabilità, igiene, ecc. Se tutte queste funzioni fossero accumulate in un solo consiglio, il cumulo di tanti affari diversi richiederebbe l'opera quotidiana di amministratori, che dovrebbero essere retribuiti. [...] Invece nel sistema federale ogni consiglio, avendo un compito limitato ed omogeneo, potrà essere formato di poche persone, [che] con qualche ora di occupazione al giorno, potranno sbrigare le loro faccende; molti impiegati vengono così ad essere eliminati e molte indennità abolite ${ }^{217}$.

Berneri sta nuovamente traendo non solo gli spunti, ma anche gran parte dei passaggi, direttamente dall'articolo salveminiano La questione di Napoli:

Se Londra avesse l'amministrazione accentrata in un unico Consiglio comunale, come Napoli, essendo la città più grande del mondo, sarebbe la città più camorrista del mondo. Invece Londra è una delle città meglio amministrate, poiché non è soggetta a un'amministrazione unitaria, ma a un'amministrazione federale.

\footnotetext{
${ }^{215}$ Rerum Scriptor [G. Salvemini], La questione di Napoli, «Critica Sociale», X, 16 dicembre 1900.

${ }^{216} \mathrm{C}$. Berneri, "Il valore civile del federalismo" cit., c. 3 .

${ }^{217}$ Ivi, cc. 3-4.
} 
Supponiamo che Napoli sia amministrata federativamente. In questo caso non formerebbe un unico Comune, ma venti, trenta Comuni secondo le condizioni topografiche della città e la configurazione degl'interessi locali. In ciascuno di questi Comuni - questo è un carattere fondamentale del sistema federativo l'amministrazione non si accentra in un unico Consiglio, ma si scinde in parecchi Consigli indipendenti, ciascuno dei quali ha il suo bilancio ed è eletto dagli interessati: il Consiglio dell'istruzione primaria eletto dai padri di famiglia, il Consiglio per l'amministrazione delle imposte dirette eletto dai contribuenti iscritti nel ruolo delle imposte, e altri Consigli per l'illuminazione, per la spazzatura, per l'igiene, per la polizia industriale, ecc.

Se tutte queste funzioni sono accumulate in un solo Consiglio, il cumulo di tanti affari richiederebbe persone occupantesi dell'amministrazione per tutto il giorno: nasce così la necessità degl'impiegati retribuiti e dei Sindaci e Assessori o benestanti o pagati dalla comunità. [...] Invece nel sistema federale ogni Consiglio, avendo per la parte sua una ristretta giurisdizione e poco lavoro, può essere formato di poche persone, che con qualche ora di occupazione al giorno possono sbrigare tutte le loro faccende; molti impiegati diventano quindi inutili, né c'è bisogno di indennità $[\ldots]^{218}$.

Berneri ritiene che sia possibile sottoporre a controllo continuo e immediato l'operato di questi «amministratori volontari» da parte degli elettori, controllo che sarebbe maggiormente possibile in un sistema federale che non in uno di tipo accentrato. Anche se questo sistema «del volontarismo amministrativo, ossia dei funzionari onorifici, potrà parere un sistema ideale ma, praticamente, impossibile», egli illustra come ciò sia già avvenuto nell'antica repubblica romana, durante il medioevo e all'interno dell'aristocrazia inglese. Chiaramente si trattava di amministrazioni non certo esemplari per trasparenza ed equità, pertanto il lodigiano afferma che

Il sistema del volontariato amministrativo non può, dunque, effettuarsi che in una società matura per un'integrale autonomia e per ben organizzato decentramento. $\mathrm{E}$ per maturità intendo, principalmente, che non sia possibile il fatto che le cariche amministrative siano affidate con un criterio politico, cioè partigiano, a scapito dell'onestà e competenza utilizzabile $\mathrm{e}^{219}$.

Possiamo notare come questo frammento sul federalismo mostri già i segni di una prospettiva politica più ampia e aperta ad apporti e influenze esterne ai classici anarchici e libertari, provenienti anche dal pensiero liberale e repubblicano. A questo proposito ci sembrano tuttavia eccessive e non condivisibili le interpretazioni di Adamo, che arriva a sostenere che la prospettiva berneriana confluisca di fatto nei valori civili liberali, e di De Maria, che tende a rappresentare Berneri come un critico della società, a metà strada tra anarchismo e liberalismo, che volle forzare e che superò l'anarchismo ${ }^{220}$. La riflessione berneriana inizia semmai, ci sembra, a connotarsi come una ricerca appunto libertaria nel senso più ampio del termine, una

\footnotetext{
${ }^{218}$ Rerum Scriptor [G. Salvemini], La questione di Napoli cit.

${ }^{219}$ C. Berneri, "Il valore civile del federalismo" cit., c. 5 . La sottolineatura è originale.
} 
ricerca capace di servirsi di spunti esterni alla tradizione anarchica che possono concorrere a rinnovarla senza con ciò snaturarla, non certo a superarla tout court $\mathrm{e}$ men che meno a farla confluire nel liberalismo.

\section{Criticare la burocrazia per criticare lo Stato}

L'8 ottobre 1920 «Umanità Nova» pubblica un articolo di Camillo Berneri sui disordini agrari e sulla miseria delle condizioni dei contadini pugliesi ${ }^{221}$. Il brano ha destato un interesse limitato da parte degli studiosi dell'anarchico lodigiano, anche perché l'argomento è insolito ed episodico nella produzione berneriana e non ha conosciuto sviluppi apprezzabili in questa fase. Vale la pena invece soffermarsi su questo articolo che esce nel pieno dei primi contatti tra Berneri e Salvemini e che nasce e si fonda evidentemente su dati e documenti raccolti dal professore pugliese, esposti in un suo intervento alla Camera e quindi pubblicati su «L'Unità» ${ }^{222}$, dimostrando ulteriormente quanto sia forte in questo frangente l'ascendente salveminiano su Berneri. L'articolo è però interessante non tanto da questo punto di vista, ma perché vi si affaccia un nuovo tema derivato da Salvemini, vale a dire quello della critica all'accentramento e allo sviluppo della burocrazia - questione da questi abbondantemente battuta e di cui, come abbiamo visto, gli unitari avevano ampiamente discusso nel progetto fondativo della «Lega democratica per il rinnovamento nazionale $\rangle^{223}$.

Lo studente anarchico sostiene nel suo articolo che la causa della miseria dei contadini pugliesi è dovuta al fatto che «il governo funziona attraverso una mastodontica ed irresponsabile burocrazia» e che

le amministrazioni comunali sono in mano a gente inetta, incapace e disonesta. L'immoralità sistematica delle burocrazie comunali, il menefreghismo poltrone, le camarille locali: è tutto un complesso di circostanze ambientali che non fanno sperare nulla nelle amministrazioni comunali e provinciali, né nelle prefetture ${ }^{224}$.

\section{Berneri prosegue sostenendo che}

Le ladronerie amministrative, i favoritismi alle clientele elettorali, le violenze dei mazzieri e degli sbirri sono state troppe perché il popolo pugliese creda ancora alla giustizia delle prefetture e all'onestà delle amministrazioni ${ }^{225}$.

${ }^{220} \mathrm{Cfr}$. P. Adamo, Introduzione a C. Berneri, Anarchia e società aperta cit., p. 17, e Carlo De Maria, Camillo Berneri cit., p. 193, ma anche Id., Camillo Berneri, un intellettuale di confine, in Camillo Berneri, singolare/plurale cit., pp. 25-30.

${ }^{221}$ C. Berneri, Pane e giustizia per le Puglie!, «Umanità Nova», I, 8 ottobre 1920.

${ }^{222}$ Vedi G. Salvemini, La provincia di Bari nel 1920, «L'Unità», IX, 16 settembre 1920, riprodotto in Id., Opere IV, Il Mezzogiorno e la democrazia italiana, vol. II cit., pp. 573-584.

${ }^{223}$ Vedi supra.

${ }^{224}$ C. B. [C. Berneri], Pane e giustizia per le Puglie! cit. ${ }^{225}$ Ibid. 
Nessuno studioso ha fino ad oggi posto attenzione al fatto che l'irresponsabilità, l'inefficienza e la corruzione dell'apparato burocratico di uno Stato centralizzato compaiano per la prima volta in questo scritto di Berneri, costituendo l'esordio di un tema e di un argomento di studio destinati a diventare presto tra i prediletti del lodigiano. Il già citato articolo Stato e burocrazia, pubblicato su «Umanità Nova» il 25 dicembre 1920, pone altri spunti interessanti per quel che riguarda il tema della lotta al centralismo. Qui Berneri ripropone la tipica argomentazione che oppone il federalismo allo Stato unitario e accentratore, con considerazioni che riprendono temi provenienti dal pensiero federalista repubblicano di età risorgimentale $\mathrm{e}$ da Pierre-Joseph Proudhon ${ }^{226}$ :

L'uniformità legislativa ed amministrativa è assurda in una nazione come è la nostra,
ove esistono così marcate differenze economiche e psicologiche fra il Nord, il
Centro e il Mezzogiorno. [...] I problemi della vita economica e sociale del popolo
italiano hanno una fisionomia propria, diversa da regione a regione, da località a
località, e richiedono ciascuno una sua specifica soluzione ${ }^{227}$.

Data questa premessa, è necessario per Berneri che la critica antistatale anarchica debba recuperare e sviluppare quella democratico-federalista emersa in età risorgimentale. Notiamo qui una sua presa di posizione importante in quanto, con le opportune differenziazioni, cerca nuovamente di coniugare il patrimonio dell'anarchismo con quello di una tradizione politica differente ma non del tutto discontinua, quella dei repubblicani federalisti, al fine di portare idee nuove nel movimento anarchico.

Dopo aver riprodotto alcuni passaggi di ispirazione salveminiana che abbiamo già analizzato nel frammento sul federalismo - nello specifico, quelli sulla lezione di civiltà e sullipotesi di un Comune non accentrato, ma organizzato in più aree autonome $^{228}$ - Berneri afferma che il concetto di autonomia della tradizione anarchica sia quello più idoneo a fornire «il substrato su cui poggerà l'edificio della politica»:

Il concetto nostro di autonomia è più ampio e più libertario di quello che hanno coloro per i quali l'autonomia rappresenta la restaurazione dell'indipendenza regionale, ma è certo che l'autonomia amministrativa e legislativa delle singole regioni è atta a favorire quella dei singoli comuni, dei singoli consigli e sindacati, sì

${ }^{226}$ Sullinfluenza di Proudhon in Italia - particolarmente su repubblicani quali Ferrari e Pisacane - si rimanda a G. Manganaro Favaretto, Proudhon in Italia. Una riflessione politica incompresa, Edizioni Università di Trieste, Trieste, 2000, soprattutto pp. 21-33. Sul tema delle continuità tra radicalismo democratico-repubblicano e primo socialismo italiano si rimanda inoltre agli studi oramai classici di F. Della Peruta, I democratici e la rivoluzione italiana, Feltrinelli, Milano, 1958, e Id., Democrazia e socialismo nel Risorgimento, Editori Riuniti, Roma, 1973. Si veda anche G. Angelini, L'altro socialismo. L'eredità democraticorisorgimentale da Bignami a Rosselli, Franco Angeli, Milano, 1999.

${ }^{227}$ C. B. [C. Berneri], Stato e burocrazia cit.

${ }^{228}$ Vedi supra. 
che si giunga ad una forma piramidale di Confederazione la cui base poggi sulla volontà associativa e la reciprocanza d'interessi dei cittadini ed il cui vertice sia rappresentato da un organo centrale di consultazione o di esecuzione, ma non di comando vero e proprio ${ }^{229}$.

Tale progetto può, secondo Berneri, del resto trovare un solco fecondo «nel pensiero federalista repubblicano di Cattaneo e di Ferrari e potrà trovare elementi di vita nel tesoro di esperienze autonomiste e federaliste che ci offre la storia dei Comuni medioevali ${ }^{230}$ : tali idee non possono forse essere sufficienti per dare un programma d'azione al movimento anarchico, ma per Berneri possono aprire la strada verso un federalismo di tipo libertario in cui gli anarchici potrebbero ritagliarsi uno spazio e giocare un ruolo da protagonisti. Interessante notare che qui, per la prima volta nella sua produzione, lo studente anarchico faccia uno specifico riferimento alle idee federaliste del repubblicano milanese Carlo Cattaneo, altra lettura e altra lezione politica che aveva già fortemente influenzato Salvemini e che certamente Berneri deriva dalla frequentazione e dalle discussioni col professore pugliese $^{231}$. Giova sottolineare che anche nel caso di Cattaneo - lo si vedrà bene nel prosieguo dello studio - come già era accaduto per Rousseau, lo studente anarchico derivi da Salvemini lo spunto, la suggestione, l'interesse di ricerca, per poi andarli a declinare e a interpretare in modo del tutto personale e originale. In definitiva, appare ancora una volta evidente come le idee e gli studi salveminiani abbiano in questo frangente un fortissimo ascendente su Berneri, come è dimostrato tra l'altro da altri articoli pubblicati su «Umanità Nova» in questo periodo ${ }^{232}$.

Il tema della critica alla burocrazia e al centralismo statale che a noi interessa ritorna in altri scritti successivi firmati da Berneri. L'articolo La crisi dello Stato,

${ }^{229}$ C. B. [C. Berneri], Stato e burocrazia cit.

${ }^{230}$ Ibid.

${ }^{231}$ Un rapido cenno a tale lascito del professore pugliese a Berneri viene fatto da G. Cerrito, Introduzione a C. Berneri, Scritti scelti, Zero in condotta, Milano, 2013, p. 24. Il recupero del federalismo di Cattaneo da parte di Salvemini è stato ben sottolineato da C. Lacaita, Salvemini interprete e continuatore di Cattaneo, in M. Degl'Innocenti (a cura di), Gaetano Salvemini e le autonomie locali, Lacaita, Manduria-Bari-Roma, 2007, pp. 53-76, e da I. Biagianti, Il federalismo di Salvemini, in G. Cingari (a cura di), Gaetano Salvemini tra politica e storia cit., pp. 202-206 e 227-230.

${ }^{232}$ Tali articoli travalicano l'interesse specifico di questa ricerca, tuttavia sono almeno da segnalare C. B. [C. Berneri], La politica della volpe di Dronero, «Umanità Nova», I, 26 ottobre 1920 e Id., La politica del ministro della malavita, «Umanità Nova», I, 27 ottobre 1920, densi di citazioni e di osservazioni che rimandano alla produzione salveminiana su «L'Unità»e al celebre saggio di G. Salvemini, Il ministro della malavita: notizie e documenti sulle elezioni giolittiane nell'Italia meridionale, Edizioni della Voce, Firenze, 1910 (nel 1919 era stata peraltro licenziata una sua seconda edizione per i tipi de La Voce, Roma), riprodotto in Id., Opere IV, Il Mezzogiorno e la democrazia italiana, vol. I cit., pp. 73-141. Anche C. B. [C. Berneri], La Dalmazia italiana?, «Umanità Nova», I, 17 dicembre 1920 contiene un esplicito rimando agli studi contemporanei di Salvemini sulla questione adriatica. 
pubblicato su «Umanità Nova» il 3 settembre $1921^{233}$, sviluppa ulteriormente l'opposizione all'accentramento e mira a sostenere la necessità che il movimento anarchico faccia propria questa lotta nella propria propaganda. Secondo Berneri «Le idee autonomiste, regionaliste, federaliste erano fino a poco tempo fa circoscritto patrimonio dei seguaci del Cattaneo e del Ferrari», ma attualmente «il movimento che si oppone al potere centrale è istintivo, con programmi molto incerti e confusi ${ }^{234}$. Lo studente anarchico torna allora a fare appello al movimento affinché studi ed elabori soluzioni concrete su questo tema - concretismo, ancora una volta invece di ribadire una propaganda fatta di soli principi antiautoritari:

Riflettere sui pericoli [del decentramento] non deve certo significare astenersi dall'incuneare la nostra propaganda autonomista e federalista nel sentimento antistatale che è oggi di viva attualità. La critica allo Stato deve oggi uscire dalle astrattezze del dottrinarismo per entrare nel campo pratico. [...] Il pericolo di fare opera riformista, col criticare lo Stato nei suoi difetti di dettaglio, nei suoi errori contingenti, ci ha distolto da una critica anti-statale che avrebbe avuto più largo seguito della solita seminagione di apriorismi programmatici, che non escono quasi mai dalla sfera degli iniziati ${ }^{235}$.

Berneri prosegue esplicitando nuovamente l'importanza di prendere spunti dall'opera di altri movimenti e gruppi politici, citando per l'appunto direttamente l'esempio di Salvemini:

Credo, ad esempio, che la campagna contro la burocrazia condotta da G. Salvemini sulla sua Unità, mentre non ha giovato a riformare la burocrazia, abbia grandemente contribuito ad allargare il senso di diffidenza e di protesta contro le bestialità e le canagliate governative, facendo opera critica più valida di una campagna anti-statale a base puramente teorica. Con questo non vengo a dire che si debbano mettere in soffitta le nostre convinzioni libertarie. Credo possibile conciliare l'affermazione teorica con la dimostrazione pratica del nostro programma, sì che siano accettati i capisaldi come logico, necessario corollario della diagnosi dello Stato $[\ldots]^{236}$.

Le idee berneriane in merito alla necessità di una critica più puntuale e concreta all'accentramento ritornano e si precisano in un altro articolo di poco successivo, intitolato Decentramento e conservazione statale ${ }^{237}$. Di nuovo viene ribadita dal lodigiano l'«importanza di un riavvicinamento alle teorie federaliste e di una critica, essenzialmente tecnica, al funzionamento ed alla struttura dei principali organi stabili», ma allo stesso tempo egli mette in guardia dal rischio che il decentramento

${ }^{233}$ C. B. [C. Berneri], La crisi dello Stato, «Umanità Nova», II, 3 settembre 1921.

${ }^{234}$ Ibid.

${ }^{235}$ Ibid. I corsivi sono nostri.

${ }^{236}$ Ibid. Il corsivo è originale.

${ }^{237} \mathrm{C}$. Berneri, Decentramento e conservazione statale, «Umanità Nova», II, 26 novembre 1921, riprodotto in Id., Il federalismo libertario, a cura di Patrizio Mauti, La Fiaccola, Ragusa, 1992, pp. 32-38. 
possa diventare «una semplice riforma statale, e non il punto di partenza per una radicale trasformazione politica ed amministrativa della vita pubblica italiana ${ }^{238}$. Secondo Berneri il semplice decentramento amministrativo non intaccherebbe affatto l'accentramento politico e pertanto tale principio sarebbe sostanzialmente conservatore, come dimostrerebbe il fatto che persino coloro che ammettono «il tramonto dell'idea dello Stato forte ed onnipotente $[. .$.$] hanno sempre avuto un sacro$ timore di spingersi troppo innanzi, di giungere cioè alla concezione autonomista, nel senso integrale della parola ${ }^{239}$. Tra gli oppositori storici all'accentramento statale lo studente anarchico cita, tra gli altri, i nomi di Farini, Minghetti, Crispi, Ferraris e dei meridionalisti Lucchini, Bertolini e Jacini, presenze insolite negli articoli di un periodico anarchico ma che servono a Berneri per trarre alcune necessarie conclusioni:

L'unità politica: ecco la preoccupazione costante dei conservatori! È questa preoccupazione che impedisce loro di sviluppare i loro criteri e progetti di decentramento e di vedere la impossibilità di un'ampia, radicale riforma amministrativa, che non sia accompagnata da un'ampia e radicale trasformazione politica.

L'accentramento statale è una condizione senza la quale il Governo non potrebbe ritardare l'epilogo del suo processo di decomposizione. Coloro che vogliono, per conservarlo, trasformare lo Stato, sono dei cattivi conservatori. Certe riforme sono come certe cure radicali. Se il corpo è giovane e robusto le sopporta e guarisce, se è vecchio e debole ne muore. [...]

Così sarà dell'idea federalista. Essa è troppo vasta per esaurirsi nei progetti, più $o$ meno ristretti del decentramento costituzionale e conservatore ed è troppo imperiosamente giusta perché non finisca per incunearsi nelle crepe della crisi statale $^{240}$.

Le valutazioni di Berneri sull'accentramento statale e sullo sviluppo di una burocrazia oppressiva e inefficiente vengono portate anche sul terreno della Russia bolscevica, che come abbiamo visto è per l'anarchico lodigiano una pietra di paragone indispensabile per studiare le problematiche che possono sorgere durante una vasta e profonda rivoluzione sociale ${ }^{241}$. Il suo articolo Stato e burocrazia in Russia del dicembre $1921^{242}$ ci sembra emblematico della necessità sentita dal giovane anarchico di portare lo studio dei problemi su un piano pratico: eccolo quindi ad analizzare proprio il tipo di apparato amministrativo messo in piedi dai bolscevichi e a mostrare come abbia nei fatti fallito nel suo proposito di smantellamento della burocrazia borghese. Secondo Berneri, le misure «proposte da Lenin contro il pericolo di una burocrazia invadente e conservatrice, intralciante il processo di auto-demolizione dello Stato comunista, hanno, data la pratica

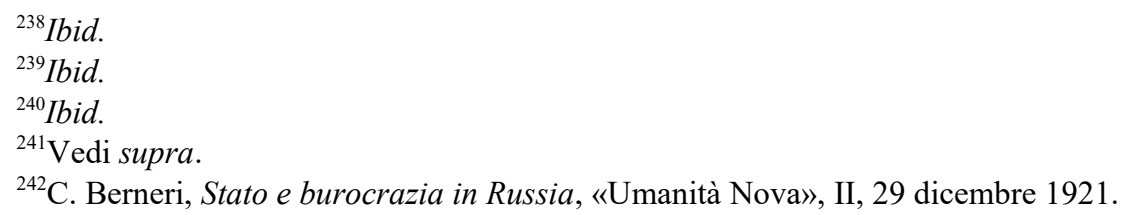


bolscevica, un valore puramente teorico» ${ }^{243}$; questa frase ci sembra serva al lodigiano non solo per criticare il carattere oppressivo dell'accentramento prodottosi in Russia, ma anche per corroborare ulteriormente la tesi per cui una serie di principi teorici non sono affatto sufficienti per elaborare e tradurre in atto un programma rivoluzionario in modo concreto e operativo.

Proseguendo nella sua analisi, lo studente anarchico sottopone a esame critico tre punti esposti da Lenin in Stato e rivoluzione (1918) per riformare l'amministrazione: l'elettività e la possibilità di rimuovere i funzionari, la retribuzione di questi ultimi parificata a quella degli operai, la loro frequente sostituzione. Il primo punto può funzionare, per Berneri, solo

in un regime di auto-democrazia, in un regime cioè che si basasse su di un sistema amministrativo e politico dal basso all'alto. Il regime bolscevico, invece, ha una ossatura completamente opposta a qualsiasi costruzione amministrativa di democrazia diretta ${ }^{244}$.

È interessante notare come in quest'ultimo passaggio ritorni, in opposizione allo Stato centrale e rappresentativo, il termine «autodemocrazia», modello politico teorizzato da Berneri già nel $1919^{245}$ e che dimostra la continuità della sua riflessione circa quelle che sarebbero dovute essere le caratteristiche di un'organizzazione rivoluzionaria fondata su centri amministrativi e politici di base similari ai soviet della Rivoluzione russa o ai clubs di quella francese.

Tornando alla critica berneriana di Lenin, i restanti due punti di Stato $e$ rivoluzione risultano all'anarchico lodigiano di difficile applicazione per via delle competenze particolari che l'attività impiegatizia richiederebbe: anche se esistono mansioni che non richiedono competenze particolari, resta il problema per cui un governo «è costretto a mantenere gli impiegati in condizioni di privilegio rispetto alle masse operaie per una necessità politica: quella di avere delle clientele»; per quanto riguarda invece il mutamento degli impiegati esso «presenta gravi difficoltà, specie per quegli uffici che richiedono un lungo tirocinio o particolari competenze ${ }^{246}$. In conclusione, secondo l'interpretazione berneriana, ciò di cui Lenin non si rende conto è che «lo Stato accentrato è la causa prima dei difetti e dell'eccessivo sviluppo della burocrazia» e che a sua volta le «irregolarità, gli abusi, le corruzioni di ogni genere della burocrazia russa sono un portato dell'accentramento amministrativo» ${ }^{247}$.

\section{Risorgimento ed eredità repubblicana: riflessioni e legami}

Parallelamente allo sviluppo del tema della critica all'accentramento statale e all'apparato burocratico quali principali questioni da studiare per dare un supporto

\footnotetext{
${ }^{243}$ C. Berneri, Stato e burocrazia in Russia cit.

${ }^{244}$ Ibid.

${ }^{245}$ Vedi supra.

${ }^{246} \mathrm{C}$. Berneri, Stato e burocrazia in Russia cit. Il corsivo è originale.

${ }^{247}$ Ibid.
} 
concreto all'opposizione anarchica allo Stato e per rendere conseguentemente l'azione del movimento maggiormente operativa e coerente, Berneri avvia una personale rilettura dell'eredità repubblicana risorgimentale e in particolare di quella corrente federalista che tante prossimità pareva avere con la sua idea di un federalismo libertario quale prima e praticabile traduzione nella realtà dell'ideale anarchico.

Il rapporto tra anarchici e repubblicani è, come abbiamo visto, complesso e meriterebbe una trattazione a parte, tuttavia potremmo sintetizzarlo nella maniera seguente: il movimento anarchico è negli anni '20 generalmente diffidente verso il PRI, giudicato troppo compromesso con le istituzioni monarchiche che dovrebbe combattere, ma mostra comunque maggiore simpatia e apertura verso le frange di militanti repubblicani legati a quella koiné, a quel patrimonio comune di idee di libertà e giustizia che si era storicamente travasato nelle prime forme di vita dell'internazionalismo italiano ${ }^{248}$. Le difficoltà di intesa tra anarchici e questa corrente repubblicana restano ad ogni modo e sono legate in particolare alla questione del cosa fare dopo la rivoluzione, di come realizzare le proprie idee, se cioè il moto rivoluzionario debba abbattere le istituzioni monarchiche per sostituirvi una costituente - e in quel caso gli anarchici si troverebbero, va da sé, sulla barricata opposta - o se si debba passare direttamente a realizzare una società di eguali organizzati secondo il principio della libera associazione - e in quel caso gli anarchici non capiscono perché ostinarsi a parlare di repubblica quando questa sarebbe già anarchia; queste tesi vengono più volte ribadite in questi anni soprattutto da Malatesta ${ }^{249}$.

È per altra via che in questa fase della sua biografia Berneri riceve suggestioni e sviluppa riflessioni e, ancora una volta, la frequentazione e l'influenza di Salvemini risultano decisivi. Dal 1920 il professore pugliese ha un nuovo studente che si sta occupando proprio del rapporto tra repubblicani e internazionalisti. Le parole di Salvemini rievocano così il ricordo di quei giorni:

Nella primavera del 1920 [...] venne a cercarmi a casa un sottotenente di artiglieria. Aveva ancora qualcosa dell'adolescente nella sua carnagione rosea e nei suoi occhi azzurri come il cielo di Firenze. Si chiamava Nello Rosselli. Era iscritto

\footnotetext{
${ }^{248}$ Vedi supra. Ad oggi mancano studi, ricerche o saggi specifici su questo complesso rapporto tra movimento anarchico e repubblicano, da segnalare tuttavia il tentativo di Papini di tracciarne un profilo circoscritto alla vicenda e alla memoria della Settimana Rossa, vedi M. Papini, Il mito della Settimana Rossa e le sue contraddizioni. Anarchici e repubblicani tra guerra e rivoluzione (1914-1921), in A. Senta (a cura di), La rivoluzione scende in strada. La Settimana Rossa nella storia d'Italia, 1914-2014. Atti del convegno di studi organizzato dall'Archivio storico della Federazione Anarchica Italiana, Imola, sabato 27 settembre 2014, Zero in condotta, Milano, 2016, pp. 47-67.

${ }^{249} \mathrm{Si}$ vedano in particolare gli articoli malatestiani rivolti al repubblicano Abussi: E. Malatesta, Noi ed i repubblicani, «Umanità Nova», I, 25 aprile 1920; Id., Noi ed i mazziniani, «Umanità Nova», I, 9 maggio 1920 e Id., Ancora sulla Repubblica, «Umanità Nova», I, 21 maggio 1920.
} 
all'università. Progettava di dedicarsi alla storia e mi domandava i miei consigli. Io conoscevo di nome, e anche un po' personalmente, sua madre [...]. "C'è un argomento," gli dissi su due piedi, "che sembra fatto proprio per te: l'ultimo periodo della vita di Mazzini dal 1860 al 1872 quando egli morì a Pisa ospite di un tuo prozio. Il primo movimento operaio sotto bandiera mazziniana e nell'Italia unificata. Apparizione dell'Internazionale bakuninista e marxista sul teatro italiano. La lotta fra Mazzini e Bakunin. Argomento bellissimo, quasi intatto. Ecco la tua tesi di laurea ${ }^{250}$.

L'allora studente Nello Rosselli tornerà pochi giorni dopo a introdurre Carlo, suo fratello, al professore pugliese; ai due giovani si unirà presto un altro compagno, Ernesto Rossi. Salvemini considererà quella compagnia «la mia nuova gioventù» ${ }^{251}$.

Scorrendo un'altra memoria salveminiana, leggiamo che

I tre amici presero l'iniziativa di fondare un piccolo Circolo di coltura fra una dozzina di persone che si riunivano qua e là nelle case dei soci. Il Circolo non era affiliato a nessuna dottrina politica. Uno dei soci riferiva su un argomento economico, politico, filosofico, e poi si discuteva. Chiunque poteva diventar socio purché accettasse il principio della libera discussione. I fascisti, quindi, erano esclusi $^{252}$.

Camillo Berneri fa parte di quella «dozzina», come ricorda anche Piero Jahier ${ }^{253}$, un altro dei fondatori del Circolo: scrivendo alla madre dell'anarchico lodigiano all'indomani della sua morte, egli ricorderà di aver conosciuto Berneri

nel dopo guerra 1918 e con Carlo Rosselli ed un gruppo nel quale emergevano Salvemini, Calamandrei, Rossi ed altri fondammo il circolo di coltura di Piazza S. Trinita che fu bruciato dai fascisti nel $1925^{254}$.

${ }^{250}$ G. Salvemini, Prefazione a N. Rosselli, Saggi sul Risorgimento e altri scritti, Einaudi, Torino, 1946, riprodotta in G. Salvemini, Opere VIII cit., pp. 718-719 (col titolo Nello Rosselli).

${ }^{251}$ Ivi, p. 719.

${ }^{252}$ G. Salvemini, Carlo e Nello Rosselli, Edizioni di Giustizia e Libertà, s.l., s.d. (ma Parigi, 1938), riprodotto in Id., Opere VIII cit., p. 676. Sul Circolo di cultura si veda P. Calamandrei, Il manganello, la cultura e la giustizia, in Non Mollare (1925). Riproduzione fotografica dei numeri usciti con tre saggi storici di Gaetano Salvemini, Ernesto Rossi, Piero Calamandrei, La Nuova Italia, Firenze, 1955, pp. 71-112.

${ }^{253}$ Piero Jahier (1884-1966) è stato uno scrittore italiano, membro tra l'altro del gruppo de «La Voce». Sarà interventista al tempo della Prima Guerra Mondiale e, in seguito, antifascista.

${ }^{254} \mathrm{La}$ lettera di Jahier è citata in A. Fochi, Con te, figlio mio!, Officina Grafica Franchising, Parma, 1948, p. 139. 
Berneri è parte attiva del Circolo di Cultura ${ }^{255}$, dove conosce, fa amicizia, inizia a confrontarsi coi fratelli Rosselli e - dato il comune tema di interesse sul rapporto tra repubblicani e internazionalisti - particolarmente con $\mathrm{Nello}^{256}$. Ne abbiamo testimonianza diretta in una lettera commovente di Amelia Rosselli, madre dei due fratelli, inviata ad Adalgisa Fochi:

Il suo caro figliolo veniva qualche volta in casa nostra e ho un ricordo indimenticabile di quelle serate piene di discussioni animate fra il suo Camillo e il mio Nello che si prolungavano sulla porta come se non potessero staccarsi $\mathrm{i}^{257}$.

La frequentazione del Circolo e dei fratelli Rosselli deve certamente costituire un ulteriore stimolo all'interesse berneriano per il pensiero democraticorepubblicano risorgimentale, ma anche il confronto col maestro Salvemini non può essere da meno: l'esame che abbiamo condotto sulle carte dell'Archivio Salvemini (conservato presso l'Istituto Storico della Resistenza in Toscana) ha fatto emergere che in quegli stessi anni '20 la ricerca storica del professore pugliese stesse tornando a concentrarsi fortemente sul Risorgimento e in particolar modo sul pensiero e l'azione di Mazzini e di Cattaneo. Sono da datarsi in questo periodo non solo la terza edizione del Mazzini ${ }^{258}$ e la raccolta Le più belle pagine di Carlo Cattaneo ${ }^{259}$, ma anche una mole considerevole di appunti per lezioni e conferenze, materiali di studio e relazioni preparatorie sui due pensatori politici risorgimentali ${ }^{260}$.

${ }^{255} \mathrm{Si}$ può vedere a tal proposito la riproduzione del Programma del «Gruppo di Cultura Politica» di Firenze, 15 aprile 1921, in appendice a C. Berneri, Epistolario inedito, vol. II, a cura di Paola Feri e Luigi Di Lembo, Edizioni Archivio Famiglia Berneri, Pistoia, 1984, p. 253. Tra gli argomenti affrontati dal Gruppo, risultano un intervento di Salvemini sulle autonomie regionali e uno di Berneri sulle idee del partito anarchico in merito alla dittatura del proletariato, mentre tra quelli in programma ve n'è uno di Jahier su Proudhon e la povertà. ${ }^{256} \mathrm{Si}$ tratta di un argomento di studio che caratterizzerà gran parte della produzione storiografica di Nello Rosselli, che troverà in Berneri un sempre interessato lettore. Su Nello Rosselli si vedano almeno A. Colombo (a cura di), I colori della libertà. Il mondo di Nello Rosselli fra storia, arte e politica, Franco Angeli, Milano, 2003, e Z. Ciuffoletti (a cura di), Nello Rosselli. Uno storico sotto il fascismo. Lettere e scritti vari (1924-1937), La Nuova Italia, Firenze, 1979.

${ }^{257}$ Lettera di Amelia Rosselli ad Adalgisa Fochi citata in F. Madrid Santos, Camillo Berneri, un anarchico italiano (1897-1937). Rivoluzione e controrivoluzione in Europa (1917-1937), Edizioni Archivio Famiglia Berneri, Pistoia, 1985, pp. 119-120.

${ }^{258} \mathrm{G}$. Salvemini, Mazzini, Edizioni La Voce, Roma, 1920.

${ }^{259}$ Le più belle pagine di Carlo Cattaneo, scelte da Gaetano Salvemini, Fratelli Treves, Milano, 1922.

${ }^{260}$ ISRT, AGS, sez. I, Manoscritti e materiali di lavoro, fsc. I, Manoscritti e materiali di lavoro dal 1898 all'esilio, $\mathrm{n}^{\circ}$ 8, Appunti e materiali di studio per corsi di lezione e conferenze su Giuseppe Mazzini; e Ivi, ${ }^{\circ} 9$, Materiali di lavoro e redazioni preparatorie per l'introduzione al libro Le più belle pagine di Carlo Cattaneo, scelte da Gaetano Salvemini. Stefano Vitali ha datato queste carte tra gli anni '10 e gli anni '20, vedi S. Vitali (a cura di), Istituto Storico della Resistenza in Toscana, Archivio Gaetano Salvemini, I, Manoscritti e materiali di 
Nello stesso periodo anche il PRI sta attraversando una fase di mutamento non indifferente: Fernando Schiavetti, nuovo segretario del partito dall'aprile 1920, sta lottando per portare il partito su posizioni socialiste e federaliste. La nuova rotta politica è emblematicamente sancita da una Circolare inviata alle organizzazioni periferiche, pubblicata sul settimanale repubblicano «L'Iniziativa», in cui i militanti vengono invitati a non avere «nessun timore di avvicinarsi troppo ai partiti rossi (anarchici, sindacalisti, socialisti)», a «scendere in mezzo ad essi con cordialità, pure essendo risoluti a non tollerare offese o diminuzioni di dignità», e a «fiancheggiare sempre la loro azione se sinceramente rivoluzionaria e in ogni caso indirizzata in senso antimonarchico» ${ }^{261}$; la nuova linea della segreteria Schiavetti uscirà infine vincitrice al Congresso di Ancona (25-27 settembre 1920) ${ }^{262}$.

In questa ridefinizione di indirizzi all'interno del PRI va peraltro emergendo la figura di Oliviero Zuccarini e la sua rivista quindicinale «La Critica Politica», pubblicata a Roma a partire dal dicembre 1920-gennaio 1921: sarà una delle voci di primo piano della corrente di sinistra del PRI, ma soprattutto inequivocabile segnale della ripresa tra i militanti repubblicani delle battaglie contro il parlamentarismo, lo Stato accentratore e la burocrazia all'interno di una visione politica organica, nonché un importante terreno di discussione e di elaborazione delle idee autonomiste e federaliste aperto a qualunque contributo da parte di altri soggetti o movimenti concordi sugli stessi temi ${ }^{263}$. Nelle Linee programmatiche, pubblicate sul primo numero della rivista ${ }^{264}$, si legge che il quindicinale vuole promuovere «un'opera di studio, di preparazione e di educazione politica» attraverso cui trovare la soluzione a quello che è ritenuto un problema storico dell'Italia:

La centralizzazione che ci fu, coll'unità, imposta $[\ldots]$ ha negato semplicemente l'Italia [...]. All'Italia, come del resto a tutti i paesi benché con maggiore ragione, si addice un regime di ampie e snodate autonomie. [...]

La soluzione $[. .$.$] non può aversi che su basi regionali e federali in uno Stato ridotto$ al minimo indispensabile degli organi, delle funzioni e delle attribuzioni. È la stessa soluzione agitata con avversa fortuna nel periodo del nostro risorgimento in nome di

lavoro, Ministero per i Beni Culturali e Ambientali - Ufficio Centrale per i Beni Archivistici, Roma, 1998, pp. 95-104.

${ }^{261}$ «L'Iniziativa», 15 maggio 1920, citata in S. Fedele, I repubblicani di fronte al fascismo (1919-1926), Le Monnier, Firenze, 1983, p. 102.

${ }^{262} \mathrm{Su}$ questa fase del Partito Repubblicano vedi S. Fedele, I repubblicani di fronte al fascismo cit., pp. 94-113, sulla figura di Fernando Schiavetti si veda invece il saggio di M. Tesoro, Un leader dissidente, in E. Signori, M. Tesoro, Il verde e il rosso: Fernando Schiavetti e gli antifascisti nell'esilio fra repubblicanesimo e socialismo, Le Monnier, Firenze, 1987, pp. 380.

${ }^{263}$ Vedi ivi, pp. 171-178, ma anche U. Chiaramonte, Il dibattito sulle autonomie nella Storia d'Italia cit., pp. 562-568, e C. Malandrino, Socialismo e libertà. Autonomie, federalismo, Europa da Rosselli a Silone, Franco Angeli, Milano, 1990, pp. 81-85. Più in generale sul personaggio cfr. inoltre F. Paolini, L'esperienza politica di Oliviero Zuccarini: un repubblicano fra Mazzini, Mill e Sorel, Marsilio, Venezia, 2003.

${ }^{264}$ Noi, Linee programmatiche, «La Critica Politica», I, 25 dicembre 1920-1 gennaio 1921. 
una idea politica repubblicana che solo grazie alla generale incoltura politica per cui si distinse l'ultimo cinquantennio poté considerarsi come superata. È la soluzione che oggi noi riprendiamo col proposito di affermarla su queste colonne e di agitarla nel paese ${ }^{265}$.

I repubblicani de «La Critica Politica» non citano apertamente i nomi di Cattaneo o di Ferrari, ma appare evidente che è proprio a quella tradizione che stanno guardando. È da notare inoltre come essi mostrino varie affinità elettive con le idee salveminiane, dall'idea di un organo di studio ed educazione politica alla critica all'accentramento statale e alla burocrazia passando per il recupero della lezione federalista cattaneana. Tra i collaboratori della rivista vi è tra l'altro anche l'autorevole repubblicano Arcangelo Ghisleri, figura cui si era a suo tempo avvicinato Gaetano Salvemini e sorta di trait d'union tra tradizione repubblicana e socialismo attraverso la rivista «Cuore e Critica», divenuta in seguito la socialista «Critica Sociale» ${ }^{266}$. Insomma, il progetto di rinnovamento agitato dal periodico di Zuccarini racchiude tutta una serie di caratteristiche e di indirizzi politici prossimi agli interessi berneriani, in particolare per quell'interpretazione del federalismo che assume, secondo Malandrino, una «colorazione socialista libertaria» ${ }^{267}$ e che, ritiene Santi Fedele, avrebbe di fatto condotto Zuccarini verso «un antistatalismo tanto accentuato da sconfinare talvolta in una sorta di utopia anarchico-libertaria dell'estinzione dello Stato ${ }^{268}$.

Il terreno è insomma fertile per le ricerche, gli studi e le riflessioni dell'anarchico lodigiano e non sorprende quindi leggere nel 1921 un suo articolo intitolato Stato $e$ Comune secondo Giuseppe Mazzini sul quotidiano anarchico «Umanità Nova» ${ }^{269}$. L'articolo è particolarmente interessante perché vi possiamo riscontrare un segno di discontinuità non solo rispetto alle critiche che nell'ambiente anarchico erano state tradizionalmente rivolte al patriota genovese, ma anche un superamento di quelle salveminiane, che opponevano recisamente l'unitarismo mazziniano al federalismo cattaneano; l'articolo mostra insomma una maturazione più profonda e autonoma del pensiero dell'anarchico lodigiano.

L'interpretazione di Berneri del pensiero mazziniano porta l'attenzione sul fatto che per il patriota genovese «la Nazione rappresentava l'associazione; il comune la libertà $\gg{ }^{270}$, pertanto anch'egli avrebbe sostenuto l'idea dell'autonomia comunale e del

\footnotetext{
${ }^{265}$ Ibid. I corsivi sono originali.

${ }^{266}$ Vedi U. Chiaramonte, Il dibattito sulle autonomie nella Storia d'Italia cit., pp. 408-419 e, più nello specifico, M. Tesoro, Democrazia in azione: il progetto repubblicano da Ghisleri a Zuccarini, Franco Angeli, Milano, 1996. Sui contatti tra Ghisleri e Salvemini si veda invece G. Salvemini, Opere IX, Carteggi, vol. I, (1895-1911), Feltrinelli, Milano, 1968, pp. 79-193. ${ }^{267}$ C. Malandrino, Socialismo e libertà cit., p. 69.

${ }^{268} \mathrm{~S}$. Fedele, I repubblicani di fronte al fascismo cit., p. 177, vedi inoltre O. Zuccarini, Tendenze e fini di un movimento autonomista, «La Critica Politica», II, 25 aprile 1922.

${ }^{269}$ C. B. [C. Berneri], Stato e Comune secondo Giuseppe Mazzini, «Umanità Nova», II, 14 settembre 1921, riprodotto in C. Berneri, Il federalismo libertario cit., pp. 110-113.

${ }^{270} \mathrm{Ibid}$.
} 
Comune come base da cui, per progressive aggregazioni, originerebbe lo Stato. Secondo lo studente anarchico, Mazzini aveva chiaro che la Nazione potesse opprimere la libera iniziativa comunale, pertanto aveva concepito il rapporto tra le due come di reciproco contrappeso, in modo da evitare tanto le tendenze autoritarie della nazione, quanto quelle centrifughe del libero comune ${ }^{271}$. Seppure le proposte mazziniane di sistemazione della penisola siano giudicate inadeguate da Berneri, vengono altresì sottolineate le perplessità espresse da Mazzini all'indomani dell'unità sulla generalizzazione delle leggi sabaude, e soprattutto il suo opporsi a chiunque ritenesse che unità fosse sinonimo di accentramento:

Secondo il Mazzini il sistema di concentrazione e di dispotismo amministrativo non ha nulla di comune con l'unità. Per questo egli si oppose al federalismo del Ferrari ed insorse contro $\mathrm{i}$ giudizi sull'unità di Proudhon. Pur riconoscendo che l'accentramento unitario era la base della conquista piemontese, cioè monarchica, del paese, ritenne necessario tale sorpasso ${ }^{272}$.

Berneri sembra accettare parzialmente l'operato del patriota, probabilmente in termini eminentemente pragmatici, poiché vi riconosce comunque una critica verso $\mathrm{i}$ problemi creati dell'assolutismo statale. A suo giudizio Mazzini aveva semmai il demerito di avere ancora una visione «trascendentale e hegeliana» rispetto alle voci migliori del fronte repubblicano, individuate ancora una volta in Cattaneo e Ferrari. Di nuovo dunque egli fa appello affinché le prospettive autonomiste e federaliste del pensiero repubblicano siano riconsiderate e messe in relazione col patrimonio teorico e programmatico del movimento anarchico:

Gli scrittori e pensatori della scuola repubblicana e democratica: da Mazzini, Cattaneo, Ferrari fino a Bovio e al Ghisleri hanno avuto il merito di elaborare la loro concezione autonomista e federalista. Credo necessario attingere a questa fonte, che se è lontana da noi sotto certi aspetti è, sotto altri, più vicina di quello che comunemente si $\operatorname{crede}^{273}$.

\section{Prosegue Berneri:}

La critica antistatale dei capi scuola dell'anarchismo è critica teorica, basata più su una concezione negativa che su un piano di indagini di esperienze positive. Non ci si può fermare alle semplici affermazioni di principio. Occorre elaborare, ampliandola e arricchendola, la nostra tesi antistatale, studiando lo Stato accentrato ed autoritario nelle sue molteplici attività e nei suoi complessi organismi. Ma occorre anche conoscere quelle teorie e tendenze parallele ed opposte

\footnotetext{
${ }^{271} \mathrm{Su}$ questi aspetti del pensiero di Mazzini si è particolarmente soffermato L. Cecchini, Unitari e federalisti. Il pensiero autonomistico repubblicano da Mazzini alla formazione del P.R.I., Bulzoni, Roma, 1974, pp. 3-43, ma cfr. anche U. Chiaramonte, Il dibattito sulle autonomie nella Storia d'Italia cit., pp. 145-152.

${ }^{272}$ C. B. [C. Berneri], Stato e Comune secondo Giuseppe Mazzini cit. ${ }^{273}$ Ibid.
} 
alle nostre. E delle teorie affini non sono da trascurarsi quelle repubblicane che, a parer mio, rappresentano per noi un solido piano di interferenza teorica oggi, e domani, un fecondo campo di comune attività pratica ${ }^{274}$.

Emerge nuovamente in questo passaggio quella che è la critica fondamentale da parte di Berneri al movimento anarchico a lui contemporaneo, ossia il sedimentarsi al suo interno dei principi teorici e l'incapacità di porsi su un piano di concreta progettualità politica. Il riferimento alla tradizione repubblicana quale esempio da prendere per la sua lunga tradizione di studio, elaborazione e rinnovamento dei principi del federalismo e dell'autonomismo, nonché come area contigua di confronto teorico e politico, sono senz'altro da ricondurre anche all'interesse in lui destato dall'apparizione del periodico di Oliviero Zuccarini.

A riprova di ciò, basterà leggere un altro articolo in cui il lodigiano tornerà a proporre nuove letture e riferimenti a movimenti e pensatori politici non anarchici. Nello scritto Gli equivoci del parlamentarismo ${ }^{275}$, dopo aver dichiarato che il parlamento eletto a suffragio universale come strumento reale di democrazia sia il «piatto di lenticchie di molte rivoluzioni», Berneri sostiene che insieme agli anarchici gli unici a denunciare apertamente questo fatto siano i «repubblicani antiparlamentari»: fa aperto riferimento al gruppo de «La Critica Politica» di Oliviero Zuccarini e al pensiero di Arcangelo Ghisleri, ma anche a una pubblicazione di Piero Calamandrei - anch'egli parte del Circolo di Cultura - in cui si mostra come gran parte delle leggi vengano redatte dalla burocrazia e come esse siano comunque «tecnicamente imperfette, farraginose, imprecise, frammentarie»: ancora una volta gli apporti per studiare e capire i problemi della realtà contemporanea sono tratti al di fuori dei classici dell'anarchismo, come a voler indicare nuovamente ai compagni quanto il movimento debba da questo punto di vista imparare e confrontarsi con altri gruppi e individui.

Tra 1919 e primi anni '20 gli anarchici rappresentano ancora in Italia una forza di primo piano e considerevole sul piano organizzativo e a livello di iniziative, ma nessuno studio sin qui condotto ha messo adeguatamente in luce come Berneri stia parallelamente partecipando e assorbendo influenze da un animato ed effervescente clima di dibattito sulle autonomie e sul federalismo e abbia dunque modo di confrontarsi con un più ampio e vivace panorama politico su questi temi: l'anarchico lodigiano si muove e si forma in un momento estremamente peculiare del dibattito politico e culturale dell'Italia del primo dopoguerra, in una fase che Malandrino ha efficacemente definito di convergenza tra «federalismo democratico e socialismo federalista ${ }^{276}$ e a cui partecipano varie esperienze, tra cui si distinguono proprio

\footnotetext{
${ }^{274}$ C. B. [C. Berneri], Stato e Comune secondo Giuseppe Mazzini cit. I corsivi sono nostri.

${ }^{275}$ Id., Gli equivoci del parlamentarismo, «Umanità Nova», II, 19 novembre 1921, riprodotto in C. Berneri, Il federalismo libertario cit., pp. 26-31 (col titolo Il sistema parlamentare e la sovranità popolare).

${ }^{276}$ C. Malandrino, Socialismo e libertà cit., pp. 58-59.
} 
«L'Unità» del maestro Salvemini e «La Critica Politica» di Zuccarini insieme alle riviste «Energie Nove» e «La Rivoluzione Liberale» dirette da Piero Gobetti ${ }^{277}$.

Non sorprende dunque che Berneri non si accontenti di quanto va agitandosi nella pubblicistica anarchica del tempo e che giunga ad auspicare che finalmente sia possibile un'apertura che arricchisca il movimento, facendolo uscire dagli assoluti schemi dottrinari e quindi arrivando a elaborare una più efficace prassi politica:

I Soviet, i Consigli di fabbrica, tutte le nuove forme di vita politica ed economica che sono uscite dal crogiuolo della rivoluzione russa trovano eco nel nostro movimento e danno adito all'esame e alla discussione. Il nostro movimento tende ad espandersi ed apre il suo pensiero alle nuove luci, e realizza progetti nuovi ${ }^{278}$.

Non è un caso allora vedere Berneri intervenire in una polemica sviluppatasi sulle colonne di «Umanità Nova» tra il repubblicano Carlo Francesco Ansaldi ed Errico Malatesta. Il dibattito si era aperto con un commento del noto militante anarchico a una lettera del giovane repubblicano pubblicata sul numero del 2 aprile $1922^{279}$ : Malatesta, commentando una lettera ricevuta da Ansaldi sulle sue intenzioni di contribuire a spostare il PRI su di un terreno «decisamente sociale» e «prevalentemente proletario e rivoluzionario», oppone i suoi dubbi circa il metodo che i repubblicani, seppur di orientamento rinnovato, intenderebbero seguire per realizzare le proprie idee programmatiche, che egli non dubita essere tendenzialmente prossime a quelle degli anarchici. Malatesta ripropone, specialmente nella seconda battuta del dibattito ${ }^{280}$, l'opposizione tra le pratiche postrivoluzionarie istituzionali - insediamento di un governo provvisorio cui fa seguito una costituente e quindi l'elezione di un nuovo governo - e quelle anarchiche, che prevedono invece l'abolizione di ogni forma di governo o di coercizione e un'organizzazione sociale basata sul libero accordo e sull'eguaglianza sociale. Malatesta riconosce che «nella pratica occorreranno dei temperamenti, poiché gli anarchici non sono la totalità della popolazione e non vorrebbero, nemmeno se potessero, imporre con la forza le loro concezioni», tuttavia si cercherebbero degli accordi di pacifica convivenza in cui «gli anarchici esigerebbero

\footnotetext{
${ }^{277}$ Vedi ivi, pp. 57-70, ma anche U. Chiaramonte, Il dibattito sulle autonomie nella Storia d'Italia cit., pp. 579-584. Berneri sarà tra l'altro in rapporto anche con Piero Gobetti, si veda a tal proposito M. Scavino, Berneri, Gobetti e la rivoluzione italiana, «Rivista Storica dell'Anarchismo», IV, n¹, gennaio-giugno 1997, pp. 73-84.

${ }^{278}$ Camillo da Lodi [C. Berneri], Considerazioni sul nostro movimento, «Libero Accordo», numero di saggio del luglio 1920, riprodotto in C. Berneri, Pietrogrado 1917 Barcellona 1937 cit., pp. 37-40, e in Id., Scritti scelti cit., pp. 60-63 (in ambedue le raccolte col titolo Il movimento anarchico). I corsivi sono originali.

${ }^{279}$ E. Malatesta, Che cos'è la repubblica sociale?, «Umanità Nova», III, 2 aprile 1922.

${ }^{280}$ C. F. Ansaldi, Repubblicanesimo sociale e anarchismo, «Umanità Nova», III, 7 aprile 1922, segue nota di Errico Malatesta.
} 
completa libertà di propaganda e di esperimentazione» lasciando gli altri liberi di seguire le vie di organizzazione che preferissero ${ }^{281}$.

Il confronto tra Ansaldi e Malatesta su «Umanità Nova» proseguirà, ma senza andare troppo oltre. Il primo affermerà in una sua replica che bisognava evitare di «ricondurre, immediatamente o troppo sollecitamente, una rivoluzione nell'ambito e nelle strettoie di una violenza giuridica», ma anche che il sistema repubblicano «se non deve considerarsi solo come una forma di governo, è tuttavia, sempre, anche una forma di governo. Sarebbe tale pure se, invece di governo, la chiamassimo, ad esempio, associazione o comunità federale, ecc.»; nello specifico egli sostiene che nella «repubblica federale-sociale» auspicata si provvederebbe a «limitare e residuare la funzione dello Stato ai compiti più necessari e, diremo, inevitabili», senza con questo pretendere di poter «attuare di un tratto tutte le innovazioni additate dal Malatesta ${ }^{282}$. Alle teorie di Ansaldi il famoso militante anarchico risponderà ribadendo fermamente la sua contrarietà a qualunque forma di governo, fosse anche la più libertaria possibile, convinto che ciò «non potrebbe che sospingere indietro la rivoluzione» portando a una legalizzazione e a un'imposizione delle nuove forme di vita sociale e quindi a nuove forme di violenza; resterà in ogni caso convinto della possibilità di dialogare e trovarsi d'accordo con repubblicani con idee similari a quelle di Ansaldi «sia nell'abbattere il regime vigente, sia nell'opporci alla immediata costituzione di un nuovo governo» ${ }^{283}$.

Camillo Berneri si inserisce a questo punto nella discussione e lo fa cercando di mediare tra posizioni anarchiche e repubblicane sociali: il lodigiano si rivolge direttamente a Malatesta e sostiene che

fra noi e i repubblicani che accettano e vogliono la rivoluzione sociale ci siano delle pregiudiziali teoriche, riguardo alle questioni economiche, che ci allontanano, ma che ci possa essere un campo di intesa pratica quando siano temperate da una parte e dall'altra le soluzioni aprioristiche, [...] che difficilmente reggono al collaudo della realtà, la quale domina le idee e spinge gli uomini su vie sempre nuove mostrando loro sempre nuovi orizzonti. Un'ampia, serena, spregiudicata discussione potrebbe facilitare questa intesa $[\ldots]^{284}$.

La risposta di Malatesta ribadirà il concetto già espresso per cui sia possibile trovare un accordo coi repubblicani di orientamento sociale, anche perché

non possiamo pretendere, e non c'illudiamo, di fare l'anarchia ed il comunismo subito e dovunque. [...] In quelle località, o regioni dove saremo forti abbastanza,

${ }^{281}$ Ibid.

${ }^{282}$ C. F. Ansaldi, Repubblicanesimo sociale e anarchismo. Consensi e dissensi sulla teoria e la tecnica della rivoluzione, «Umanità Nova», III, 14 aprile 1922, segue nota di Errico Malatesta. I corsivi sono originali.

${ }^{283}$ Ibid.

${ }^{284} \mathrm{C}$. Berneri, Repubblicanesimo sociale ed anarchismo. In margine alla polemica AnsaldiMalatesta, «Umanità Nova», III, 16 aprile 1922, segue nota di Errico Malatesta. I corsivi sono nostri. 
ignoreremo qualsiasi governo si faccia altrove, e se volesse imporsi a noi, lo combatteremmo come un invasore straniero.

Allora avremo una «patria», la patria della rivoluzione, e sapremo difenderla ${ }^{285}$.

Il prosieguo del dibattito tra Ansaldi e Malatesta non modificherà sostanzialmente i termini del discorso: al repubblicano che invita gli anarchici a partecipare e a contribuire a un'eventuale repubblica sociale che possa progressivamente favorire e preparare un passaggio all'anarchia, l'autorevole militante anarchico continuerà a opporre il netto rifiuto di qualunque nuova forma di governo e a domandare se la repubblica sociale dovrà nascere per mezzo di una costituente - cioè per mezzo di mezzi quali la delega e l'imposizione della maggioranza sulla minoranza - o se invece non sia altro che un nome diverso per quell'ordine sociale che egli chiama anarchia ${ }^{286}$.

$\mathrm{Ci}$ appare tuttavia possibile affermare che Berneri, nonostante il noto debito di influenza che ha anche con Malatesta, non sia affatto soddisfatto dello sviluppo del dibattito, di questo continuo arenarsi del discorso sulle tipiche pregiudiziali anarchiche, e che inizi quindi a pensare che si sarebbe dovuto e potuto fare di più da parte degli anarchici per incidere sulla realtà e determinare il corso dei possibili eventi rivoluzionari verso la realizzazione dei propri propositi. Guardare a un campo repubblicano che pare sempre più nettamente e decisamente caratterizzato da contenuti socialisti e federalisti, specialmente all'indomani del Congresso di Trieste (22-25 aprile 1922 $)^{287}$, deve certo apparirgli un'opzione da non scartarsi a priori.

Le idee di Malatesta sono coerenti con la concezione gradualista da lui sviluppata $^{288}$ e l'anarchico lodigiano è certo ben lungi dal rifiutarle, ma ci sembra nondimeno che in lui si faccia fortemente avanti in questo periodo il timore che l'intransigenza dei compagni potrebbe relegare il movimento a una posizione marginale o subalterna in un eventuale contesto rivoluzionario.

\section{Programma minimo federalista, un pratico proposito}

L'osservazione sull'andamento degli eventi successivi al Biennio Rosso e il progressivo montare dell'ondata nera fascista non possono non aver condotto lo

\footnotetext{
${ }^{285}$ Nota di Errico Malatesta a C. Berneri, Repubblicanesimo sociale ed anarchismo cit. ${ }^{286}$ Vedi C. F. Ansaldi, Repubblicanesimo sociale e anarchismo, «Umanità Nova», III, 26 aprile 1922; E. Malatesta, Repubblicanesimo sociale e anarchismo, «Umanità Nova», III, 27 aprile 1922; C. F. Ansaldi, Repubblicanesimo sociale e anarchismo, «Umanità Nova», III, 29 aprile 1922, segue nota di Errico Malatesta.

${ }^{287} \mathrm{Si}$ veda S. Fedele, I repubblicani di fronte al fascismo cit., pp. 200-210.

${ }^{288}$ Malatesta stava raggiungendo proprio in questo periodo la maturazione di tali idee, ma lo farà in particolar modo tra 1924 e 1926. Si veda a tal proposito G. Berti, Errico Malatesta e il movimento anarchico italiano e internazionale, 1872-1932, Franco Angeli, Milano, 2003, pp. 755-789.
} 
studente anarchico ad approfondire la sua prospettiva sul proprio movimento e su quella che avrebbe dovuto essere la sua azione. $\mathrm{Ci}$ sembra chiaro che in questo frangente il lodigiano stia iniziando a sviluppare una visione e una linea politica che riescano a coniugare alla tradizione del suo partito la lezione concretista e antidogmatica mutuata dal suo maestro Salvemini, una lezione che lo ha oramai condotto a una sempre più spiccata critica del fossilizzarsi del movimento anarchico sulle proprie posizioni. Come si è mostrato, per Berneri sono importantissimi lo studio dei problemi, unitamente al confronto e al dibattito sui principi dell'anarchismo stesso, per superare l'ancora imperante ragionare per assoluti e le antiche pregiudiziali politiche pretenziosamente ritenute indubitabili, valide una volta per tutte. L'anarchismo avrebbe dovuto invece procedere diversamente, misurandosi e confrontandosi con i problemi e i dati reali cui si trovava di fronte, rinunciando alla pretesa di realizzare immediatamente il proprio "programma massimo" e accettando un compromesso tra il contingente e le proprie aspirazioni.

In onore a questa attitudine - che abbiamo chiamato concretismo rivoluzionario - la traduzione più immediata sul piano pratico del programma anarchico inizia allora a essere individuata da Berneri in un progetto federale di ispirazione libertaria che possa trovare una sua progressiva realizzazione confrontandosi coi fatti, coi diversi problemi pratici del momento, accettando il contributo e il confronto con altre forze politiche sensibili alla questione.

\section{Un dibattito tra anarchici}

La dimostrazione dell'emergere di questi nodi del pensiero berneriano appare con chiarezza nel 1922 all'interno di un dibattito sul quindicinale milanese «Pagine Libertarie». La polemica era stata sollevata da Carlo Molaschi ${ }^{289}$, direttore del periodico, in relazione a un articolo di Luigi Damiani ${ }^{290}$ apparso in due puntate su «Umanità Nova» ${ }^{291}$ e teso a porsi sostanzialmente lo stesso problema che Berneri ha

\footnotetext{
${ }^{289}$ Carlo Molaschi (1886-1953) è stato un importante pubblicista anarchico, inizialmente esponente della corrente individualista e quindi, soprattutto a partire dalla fondazione di «Umanità Nova», sempre più prossimo al movimento organizzato. Fu direttore di diversi periodici, tra cui «Il Ribelle», «Cronaca libertaria» e «Nichilismo», nonché fondatore della libreria «Tempi nuovi». Vedi «Molaschi, Carlo», in M. Antonioli et al. (diretto da), Dizionario biografico degli anarchici italiani, vol. II, Edizioni Biblioteca Franco Serantini, Pisa, 2004, pp. 194-195.

${ }^{290}$ Luigi "Gigi” Damiani (1876-1953) è stato un anarchico italiano. Emigrato in Brasile sul finire del XIX secolo, partecipa attivamente al movimento locale e ne diventa un importante esponente. Espulso nel 1919, rientra in Italia dove collabora alla stampa anarchica locale, specialmente su «Umanità Nova». Fonderà nel 1923 il settimanale «Fede!» che porterà avanti fino al 1926, quando inizia anche per lui un lungo periodo da fuoruscito che si concluderà nel 1946. Vedi «Damiani, Luigi», in M. Antonioli et al. (diretto da), Dizionario biografico degli anarchici italiani, vol. I, Edizioni Biblioteca Franco Serantini, Pisa, 2003, pp. 481-484.
} 
mostrato di avere a cuore da diverso tempo: come portare il movimento anarchico su un terreno pratico, smuovendolo dal suo isolamento e dalla sua marginalizzazione.

Commentando tale articolo, Molaschi esprime dei dubbi:

Colle idee di Gigi Damiani io ho avuto, ed ho, molti punti di contatto. [...]

I suoi ultimi articoli pubblicati su Umanità Nova però prospettano, come conclusione, delle idee che io non condivido. Mi pare parli di revisione di dottrine e di anarchismo italiano... Si dovrebbe arrivare ad una revisione delle idee fondamentali anarchiche lanciate da Bakounine, da Kropotkine, da Reclus e da altri, tenendo presente quelle di Carlo Cattaneo, di Giuseppe Ferrari, di Carlo Pisacane. Gigi Damiani non dice chiaramente questo, ma lo lascia indovinare dal lettore intelligente ${ }^{292}$.

Proseguendo, Molaschi sostiene che

nessuna revisione aspetta l'anarchismo. Il buon anarchismo di cinquant'anni or sono è ancora in efficienza. Bakounine è ancora il maestro incontrastato. [...]

Torniamo alle fonti teoriche e seguiamo la via maestra. La via maestra non sbaglia mai, va dritta alla meta ${ }^{293}$.

Luigi Damiani risponderà su «Pagine Libertarie» a Carlo Molaschi affermando effettivamente di ritenere necessaria la ricerca di contatti in tutte quelle correnti dei socialisti e dei repubblicani più prossime al federalismo, non mancando di criticare l'operato e le idee di cui si fa propaganda entro l'ambiente anarchico - l'operaismo e il dottrinarismo su tutti. Molaschi ribadirà per contro che l'anarchismo non necessiti di alcuna revisione e che sia anzi necessario restare «fedeli al buon anarchismo di cinquant'anni or sono che è sempre giovane, gagliardo, pieno di promesse per il vicino domani» ${ }^{294}$.

Nel numero del 20 novembre il dibattito sul federalismo si fa più vivace e tra le voci figura anche quella di Camillo Berneri, un cui intervento è pubblicato sotto il titolo Anarchismo e federalismo ${ }^{295}$. L'anarchico lodigiano, lo abbiamo visto, stava da

${ }^{291}$ Gigi Damiani [L. Damiani], Prima di ricominciare...per ricominciare, «Umanità Nova», III, 2 e 9 settembre 1922.

${ }^{292}$ Charles L'Ermite [C. Molaschi], Sulla situazione (pensieri e commenti), «Pagine Libertarie», II, 21 settembre 1922. Il corsivo è originale.

${ }^{293}$ Ibid.

${ }^{294}$ Charles l'Ermite [C. Molaschi], Postilla a Gigi Damiani [Luigi Damiani], Revisione, «Pagine Libertarie», II, 20 ottobre 1922, citato da Pietro Adamo nella nota introduttiva a C. Berneri, Anarchismo e federalismo, in Id., Anarchia e società aperta cit., pp. 108-109.

${ }^{295}$ C. Berneri, Anarchismo e federalismo - Il pensiero di Camillo Berneri, «Pagine Libertarie», II, 20 novembre 1922. L'articolo è riprodotto in Id., Pietrogrado 1917 Barcellona 1937 cit., pp. 53-56 (col titolo Contributo ad un dibattito sul federalismo), in Id., Il federalismo libertario cit., pp. 39-42, in Id., Anarchia e società aperta cit., pp. 109-112, e in Id., Scritti scelti cit., pp. $74-77$ (anche qui col titolo Contributo ad un dibattito sul federalismo). 
tempo riflettendo su questo tema: addirittura in quello stesso novembre 1922 aveva manifestato, in una lettera al militante e storico dell'anarchismo Max Nettlau, l'intenzione «di fare uscire un periodico di propaganda federalista» ${ }^{296}$. Nel suo articolo su «Pagine Libertarie» Berneri ha così l'occasione di ritornare sull'argomento e la prima cosa che gli preme è non a caso quella di criticare Molaschi che, rispondendo a Gigi Damiani sull'avvicinamento al federalismo sovversivo, aveva sottolineato l'assenza di simili elementi in campo repubblicano, a causa della forte e ancora dominante influenza unitaria di Mazzini. Invece, sostiene Berneri:

Che la generalità dei repubblicani abbia seguito, e segua tuttora, Mazzini, invece di Ferrari e Cattaneo, è vero, ma è anche vero che vi è un forte gruppo di repubblicani che continuano la tradizione federalista, arricchendola ed elaborandola. Basta, per esempio, la lettura della rivista «La critica politica» per convincersene ${ }^{297}$.

Alla luce della nostra ricostruzione, il riferimento alla rivista di Oliviero Zuccarini e alla sua opera di aggiornamento e riflessione sul federalismo repubblicano appaiono tutt'altro che sorprendenti. Ma l'intento berneriano è soprattutto quello di attaccare l'integralismo anarchico di Molaschi e, ancora una volta, la pietra di paragone sono proprio i repubblicani:

I repubblicani federalisti hanno, bisogna riconoscerlo, fatto molto più di noi, nel campo teorico! Noi siamo ancora al federalismo di Bakunin, che a Molaschi pare, a quanto sembra, non plus ultra. E questo è un grave segno. Dimostra che non abbiamo fatto che pochi passi più in là dei Maestri.

Molaschi, opponendosi al revisionismo, dice: «Rimaniamo fedeli al buon anarchismo di cinquant'anni or sono che è sempre giovane, gagliardo, pieno di promesse pel vicino domani». Bisogna intenderci: l'anarchismo di cinquant'anni or sono è sempre giovane, $[. .$.$] nel senso che contiene delle verità che sono ben lontane$ dall'essere smentite, anzi rifulgono di nuova luce sullo sfondo dei fatti. Ma le ideologie di cinquant'anni fa sono sorpassate ${ }^{298}$.

Per Berneri gli anarchici si accontentano del loro patrimonio ideologico e mancano pertanto di consapevolezza nei confronti dei problemi attuali, perseverando a ripetere il verbo della letteratura classica di propaganda. Invece l'anarchismo dovrebbe seguire l'esempio che arriva da altre correnti politiche - quelle dei repubblicani federalisti, in questo caso - e farsi più vasto e attuale attraverso un

${ }^{296}$ Lettera di Camillo Berneri a Max Nettlau (Firenze, 6 novembre 1922), in C. Berneri, Epistolario inedito, vol. II cit., p. 19.

${ }^{297}$ C. Berneri, Anarchismo e federalismo cit. Lo stesso Luigi Damiani aveva risposto a Molaschi che «nel partito repubblicano si va sviluppando una corrente di sinistra che dovrà necessariamente sfociare nel federalismo; tendenza che noi dovremmo incoraggiare». Vedi Gigi Damiani [L. Damiani], Anarchismo e federalismo - Le conclusioni di Gigi Damiani, «Pagine Libertarie», II, 20 novembre 1922. Il corsivo è originale.

${ }^{298}$ Ibid. 
rinnovamento che non implichi con ciò la perdita dei suoi principi. L'anarchico lodigiano usa un'immagine evocativa in tal senso:

Come nelle ricerche scientifiche l'ipotesi può illuminare la strada delle indagini, quando si sia capaci di spegnere questa luce se essa risulta falsa, l'anarchismo deve conservare quel complesso di principi generici che costituiscono la base del suo pensiero e l'alimento passionale della sua azione, ma deve sapere affrontare il complicato meccanismo della società odierna senza occhiali dottrinari e senza eccessivi attaccamenti all'integrità della sua fede ${ }^{299}$.

Berneri ritorna dunque a insistere su un tema già sviluppato in precedenza, vale a dire la necessità di far posto a nuove forme di critica allo Stato che vadano al di là del semplice principio antistatale. Il lodigiano si lancia così in un'affermazione audace, considerando che viene dalla mente di un militante anarchico:

Il nemico è là: lo Stato. Ma lo Stato non è solo un organismo politico, strumento di conservazione delle ineguaglianze sociali; è anche un organismo amministrativo. Come impalcatura amministrativa lo Stato non si può abbattere. Si può cioè smontare e rimontare, ma non negarlo, poiché ciò arresterebbe il ritmo della vita della nazione, che batte nelle arterie ferroviarie, nei capillari telefonici, ecc.

Federalismo! È una parola. È una formula senza contenuto positivo. Che cosa ci dicono i Maestri? Il presupposto del federalismo: la concezione antistatale, concezione politica e non impostazione tecnica, paura dell'accentramento, e non progetti di decentramento ${ }^{300}$.

Affinché sia possibile portare questa critica ed elaborare progetti concreti che siano alternativi a un'amministrazione centralizzata, inefficiente e irresponsabile è necessario, per Berneri, liberarsi dalle «formulette complicate», dai «barattoli pieni di fumo», dai «chiacchieroni» e dai «semplicisti».

La chiosa dell'articolo evidenzia in modo chiaro questa sua posizione che ci appare sempre più chiaramente come la risultante di una personale rielaborazione dalla lezione concretista e problemista di Salvemini:

Bisogna cercare le soluzioni affrontando i problemi. Bisogna che ci formiamo un nuovo abito mentale. Come il naturalismo superò la scolastica medioevale leggendo nel gran libro della natura invece che sui testi aristotelici, l'anarchismo supererà il pedante socialismo scientifico, il comunismo dottrinario chiuso nelle sue caselle aprioristiche, e tutte le altre ideologie cristallizzate.

Io intendo per anarchismo critico un anarchismo che, senza essere scettico, non s'accontenta delle verità acquisite, delle formule semplicistiche, un anarchismo idealista ed insieme realista, un anarchismo, insomma, che innesta verità nuove nel tronco delle sue verità fondamentali, sapendo potare i suoi vecchi rami ${ }^{301}$.

${ }^{299}$ C. Berneri, Anarchismo e federalismo cit.

${ }^{300} \mathrm{Ibid}$. Il corsivo è nostro.

${ }^{301}$ Ibid. 
Berneri in sostanza auspica non solo un aggiornamento dei principi, ma anche che da questi si possa passare alla pratica. Egli pone la priorità nella questione di come organizzare nella prassi lo Stato in senso federale e libertario rispetto al perché organizzare nella teoria lo Stato in senso federale e libertario. Le affermazioni di principio non sono più sufficienti, è necessario portare a successiva elaborazione la tesi antistatale e farne un contenuto più concreto e credibile della propaganda anarchica.

La risposta di Molaschi - teniamolo a mente - è di netto rifiuto rispetto alle tesi esposte dal lodigiano, specie quelle riguardanti l'impossibilità di negare lo Stato anche solo come ente amministrativo:

Il revisionismo di Berneri minaccia di condurci dritti dritti al socialismo, a quel socialismo autoritario ed accentratore contro il quale noi agitiamo l'idea federalista libertaria.

Il federalismo di Bakounine è vecchio [...] ed è incompleto. Esso è politico e non tecnico... Così dice il Berneri. D'accordo. Ed io quando dico di agitare l'idea bakouniniana, non intendo accettarla in blocco, senza ritocchi. Aggiorniamola pure. Ma lasciamo la base che è il principio anti-autoritario e libertario della rivoluzione. Accettiamo tutta la negazione dello Stato e non pensiamo a nessuna trasformazione statale sia pure amministrativa ${ }^{302}$.

\section{Su due assiomi libertari: tolleranza e libertà}

Il tentativo di aggiornare le idee e le pratiche anarchiche troveranno di lì a poco uno spazio considerevole nel settimanale «Fede!», fondato nel settembre 1923 e diretto da Luigi Damiani: levando l'ancora del suo progetto giornalistico, egli dichiarerà di collocarsi all'interno del «vasto programma della concezione anarchica di un nuovo assetto sociale», ma precisando che «nel tracciato rigido e colossale sono dei vuoti, [...] dei punti che bisogna chiarire, [...] dettagli o trascurati o applicati con poco discernimento» ${ }^{303}$.

Tra i contributi che Berneri darà a questa rivista, ve ne sono due particolarmente interessanti che, anche se non direttamente attinenti col tema del federalismo libertario, dimostrano quanto le idee politiche berneriane si stessero facendo più ampie e complesse ma tutto sommato coerenti con l'idea di un ordine che avesse quali criteri politici di riferimento l'autonomia e il federalismo. In un articolo del 20 aprile $1924^{304}$ il lodigiano sostiene che «L'anarchia è la filosofia della tolleranza»e argomenta la sua tesi come segue:

${ }^{302}$ C. Molaschi, Anarchismo e federalismo - Postilla del compilatore, «Pagine Libertarie», II, 20 novembre 1922.

${ }^{303}$ G. D. [L. Damiani], Il nostro programma. Parole che interessano amici e nemici, «Fede!», I, 16 settembre 1923.

${ }^{304}$ C. B. [C. Berneri], Della tolleranza, «Fede!», II, 20 aprile 1924, riprodotto in C. Berneri, Il federalismo libertario cit., pp. 114-117, e in Id., Anarchia e società aperta cit., pp. 117-120. 
Il nostro internazionalismo è basato sul principio della possibilità di pacifica convivenza di vari gruppi etnici aventi una lingua, una storia, usi, costumi diversi. Così la nostra concezione di assoluta libertà di stampa, di parola, d'insegnamento è basata sulla convinzione che non siano dannose varie e contrastanti correnti di pensiero, quando queste si correggano reciprocamente nel libero gioco della loro concorrenza. Anche nel campo economico, la nostra tolleranza si afferma, riguardo all'artigianato di fronte alla grande industria, alla piccola proprietà rurale di fronte all'agricoltura collettiva. Noi siamo i liberisti del socialismo appunto per questa fiducia nella possibilità di fusione degli estremi, di soluzione armonica degli opposti ${ }^{305}$.

Riteniamo che l'ampia tolleranza e la capacità di far convivere persone, idee e soluzioni diverse di cui parla Berneri possano costituire come un corollario ideale del suo progetto federalista libertario, un corollario attraverso cui rimuovere ogni alone mitologico dall'anarchia: essa «non sarà la società dell'armonia assoluta, ma la società della tolleranza» ${ }^{306}$.

E se di tolleranza si tratterà, se non sarà questione di un'utopica e ideale armonia assoluta, ma di concreta possibilità di apertura a diversi individui e a diversi modi di concepire le relazioni sociali ed economiche, bisognerà anche ripensare e riconsiderare uno dei capisaldi teorici del pensiero anarchico, quello dell'antiautoritarismo, ovvero del valore assoluto e indiscutibile del principio di libertà. Nell'articolo Libertà ed autorità, pubblicato sempre su «Fede!» il 22 giugno $1924^{307}$, Berneri sostiene che «non può darsi l'idea di libertà, bensì tante idee di libertà. Così nella realtà pratica non vi è la libertà, ma tante libertà ${ }^{308}$. Il rapporto tra libertà e autorità è dunque più complesso di quanto le dichiarazioni di principio della letteratura anarchica non abbiano mai ammesso:

La libertà cosciente $[\ldots]$ non è assenza di ostacoli, né come fatto né come coscienza di esso. $[\ldots]$

La libertà umana è capacità di sorpassare ostacoli, interni od esterni, e di cercarseli.

$[\ldots]$

Il processo evolutivo della libertà presuppone ed implica lo sforzo. L'ostacolo che dà la possibilità della liberazione è, nel caso nostro, l'autorità ${ }^{309}$.

La libertà cresce e si sviluppa solo in quanto è presente un'autorità che la accetti e la limiti. Nel discorso berneriano, tale autorità si manifesta nello specifico anche

${ }^{305}$ C. B. [C. Berneri], Della tolleranza cit. Interessante notare che, in materia economica, Berneri avesse definito gli anarchici come «i liberali del socialismo» in quanto avversi alle soluzioni comuniste ritenute centralizzatrici e autoritarie, vedi C. Berneri, Il liberalismo nell'Internazionale, «La Rivoluzione Liberale», II, 24 aprile 1923.

${ }^{306}$ Ibid.

${ }^{307}$ C. Berneri, Libertà ed autorità, «Fede!», II, 22 giugno 1924, riprodotto in Id., Il federalismo libertario cit., pp. 118-120, e in Id., Anarchia e società aperta cit., pp. 121-123. ${ }^{308}$ Ibid.

${ }^{309} \mathrm{Ibid}$. Il corsivo è originale. 
come libertà degli altri, rendendo il rapporto tra libertà e autorità ancora più dinamico:

Ma la mia libertà è la nostra libertà, sia perché è condizionata alle capacità di volere della società in cui vivo, nonché di quelle che l'hanno preceduta e con essa convivono, sia perché la coscienza della mia libertà [...] è nata dal contrasto con le libertà altrui, e in quel contrasto s'è potenziata e in esso tende a definirsi sempre più. Tra le libertà in conflitto con la mia vi è l'autorità: quella dei genitori, quella del maestro, quella del libro, ecc. Ed è, d'altra parte, l'eteronomia dell'autorità, quando non mi ha soffocato od offuscato lo spirito, che ha permesso la mia autonomia, cioè la mia libertà ${ }^{310}$.

Appare chiaro come agli occhi di Berneri il vago antiautoritarismo anarchico rappresenti di fatto un non senso, poiché è proprio il rapporto che la libertà instaura anche con l'autorità - non già l'eliminazione di essa - che permette di rafforzare l'autonomia, la capacità di darsi da soli le proprie regole senza subirle dall'esterno ${ }^{311}$. La similitudine con quelle che furono le considerazioni di un altro anarchico, Proudhon $^{312}$, circa l'antinomia tra libertà e autorità è considerevole: anche per il francese il principio di libertà non poteva espandersi illimitatamente fino ad annullare il principio di autorità; quest'ultimo poteva indebolirsi, restringersi, ma mai scomparire.

Più concretamente, Berneri coglie come anche nella società anarchica possa e debba sopravvivere una certa forma di autorità; si tratta semplicemente di concepire in modo diverso il principio di autorità:

Il nostro anti-autoritarismo è rivolto contro l'autorità che non permette $[\ldots]$ il processo di emancipazione. [...]

${ }^{310} \mathrm{Ibid}$. Su questo rapporto tra eteronomia e autonomia si è particolarmente soffermato $\mathrm{S}$. D'Errico, Anarchismo e politica. Nel problemismo e nella critica all'anarchismo del ventesimo secolo, il "programma minimo" dei libertari del terzo millennio. Rilettura antologica e biografica di Camillo Berneri, Mimesis, Milano, 2007, pp. 275-278.

${ }^{311}$ Berneri non è peraltro nuovo a queste idee e già su «Umanità Nova» aveva sostenuto che «La libertà non consiste nel fare quello che si vuole, ma nel sapere quello che si deve fare e nel fare quello che si sa che si deve fare. Cioè, in parole più chiare, l'uomo libero non è quello che si afferma nella vita senza curarsi di sapere se la sua volontà è diretta bene, ma è l'uomo che cerca nel fondo della propria coscienza la via da seguire e quando l'ha scoperta sa andare lungo essa anche se il proprio interesse [...] non si concilia con l'indirizzo dato alla vita propria. [...] La vera volontà si esercita, dunque, nell'essere padroni di noi. È più facile spezzare la volontà di mille che non di noi stessi. [...] La volontà di predominio non forma l'uomo quanto la volontà di dominio su se stessi». Vedi C. Berneri, A proposito di libertà, «Umanità Nova», III, 23 settembre 1922. I corsivi sono nostri.

${ }^{312}$ Vedi P.-J. Proudhon, Du Principe fédératif et de la nécessité de reconstituer le Parti de la Révolution, E. Dentu, Paris, 1863, tr. it. Mondo Operaio - Edizioni Avanti!, Roma, 1979, pp. $5-13$. 
La superiorità dell'anarchismo consiste in una migliore concezione dell'autorità, e da questa bisognerebbe partire, nella propaganda.

$[\ldots]$

All'autorità delle gerarchie basata sulla violenza e sul privilegio anteponiamo quella delle gerarchie tecniche, agenti per utilità generale e formatesi liberamente.

All'autorità formale del grado e del titolo anteponiamo l'autorità reale del valore e della preparazione individuali ${ }^{313}$.

In sintesi, secondo Berneri

Autorità e libertà sono termini di un rapporto antitetico che si risolve in sintesi, tanto più l'antitesi è voluta. [...]

L'anarchia mi pare risulti dall'approssimarsi, identificarsi mai, ché sarebbe la stasi, della libertà e dell'autorità. Come principi. Come fatti, libertà e autorità stanno tra loro come verità ed errore; come enti che differenziano e si identificano, nel divenire storico $^{314}$.

L'abolizione totale dell'autorità non è dunque pensabile, si può e si deve invece riconoscere un ruolo diverso e dinamico dell'autorità per gettare le basi di un'organizzazione sociale anarchica che non sia un'astratta idealizzazione o un fumoso ologramma, ma che rappresenti una via alternativa a quella vigente praticabile a tutti gli effetti.

\section{Il federalismo libertario come programma "minimo" degli anarchici}

Malatesta, per parte sua, sul quindicinale «Pensiero e Volontà» continuerà a dichiararsi contrario a condividere e a supportare un movimento rivoluzionario di carattere repubblicano, sia pure di ispirazione libertaria, sociale e federalista. Nonostante il regime fascista sia oramai realtà, nonostante le libertà costituzionali siano sempre più in pericolo, nonostante sia necessario opporsi all'autoritarismo, resiste e si ripropone ancora per Malatesta la distinzione del «fatto rivoluzionario [...] dai governi che vengono dopo ad arrestare la rivoluzione ed a sopprimere il più che possono delle conquiste rivoluzionarie» attraverso l'uniformità e la coercizione della legge $\mathrm{e}^{315}$.

Per ironico che possa sembrare, è invece la voce di Carlo Molaschi - oppositore, lo ricorderemo, delle proposte berneriane - a essere una di quelle più convinte della necessità di adeguare le aspirazioni anarchiche alle contingenze tattiche del momento, di far sì che il movimento anarchico stia nel gioco e non fuori dal gioco dell'opposizione politica al fascismo: giacché gli anarchici difficilmente riusciranno a essere la forza più influente dell'antifascismo, essi dovranno «appoggiare quella risoluzione che più d'ogni altra si avvicina ai nostri principi» e soprattutto «insistere

${ }^{313}$ C. Berneri, Libertà ed autorità cit.

${ }^{314}$ Ibid.

${ }^{315}$ E. Malatesta, Repubblica e rivoluzione, «Pensiero e Volontà», I, 1 giugno 1924. Tali idee vengono ribadite nel numero successivo, vedi Id., Ancora di repubblica e rivoluzione, «Pensiero e Volontà», I, 15 giugno 1924. 
perché il nuovo ordinamento sia fatto su basi federali piuttosto che accentratrici»; per Molaschi sarebbe una soluzione transitoria, ma quanto meno in essa ci sarebbero «maggiori possibilità di influire sulla vita politica e sociale della nazione in modo da sospingere sempre più l'ordinamento verso le nostre concezioni» ${ }^{316}$.

Molaschi sarà prolifico di articoli sul tema del federalismo libertario e sulla necessità tattica per gli anarchici di aggiornare i propri principi e di accettare di prendere parte a quella corrente antifascista di area democratica - individuata soprattutto nei gruppi di «Rivoluzione Liberale» e de «La Critica Politica» ${ }^{317}$ guidata da «spirito libertario», certo che «il pensiero di Carlo Pisacane e le dottrine di Giuseppe Ferrari sono più vicine all'anarchismo che non le dottrine del socialismo scientifico di marca tedesca ${ }^{318}$. Le parole di Berneri, come si può constatare, non sono state gettate al vento e qualcuno all'interno del movimento pare averle comprese e recepite. Molaschi le spinge anzi apertamente alla estreme conseguenze:

Dare libertà ed autonomia ai comuni e alle regioni, fiaccare la burocrazia statale decentrando il potere, diminuire la potenza dell'oligarchia levandole l'appoggio della forza armata, fare che lo Stato sia il meno-Stato... [...]

Se non è possibile - per ragioni più forti del nostro volere - demolire lo Stato, perché non cercare di diminuirlo attraverso il decentramento? Perché non incominciare ad abituare gli uomini a far senza dello Stato, favorendo un ordinamento sociale che $[. .$.$] può almeno facilitare l'avvento dell'anarchia { }^{319}$ ?

Il federalismo libertario come aspetto operante del pensiero anarchico qui espresso da Molaschi è senza dubbio di derivazione berneriana, come lo è la considerazione successiva per cui gli anarchici potranno influire in maniera determinante sugli eventi rivoluzionari solo se si doteranno di un programma chiaro e se si libereranno

${ }^{316} \mathrm{C}$. Molaschi, Dalla teoria alla realtà, «Fede!», II, 17 agosto 1924.

${ }^{317}$ Le due riviste hanno ulteriormente sviluppato il dibattito politico e culturale intorno al federalismo, l'autonomismo, l'autogoverno e il sindacalismo, giungendo a originali elaborazioni di democrazia integrale, si veda a questo proposito U. Chiaramonte, Il dibattito sulle autonomie nella Storia d'Italia cit., pp. 657-666 e C. Malandrino, Socialismo e libertà cit., pp. 75-85.

${ }^{318} \mathrm{C}$. Molaschi, Le idee federaliste nell'attuale crisi italiana, «Pensiero e Volontà», I, 15 ottobre 1924, i corsivi sono originali. La produzione di Molaschi sul tema del federalismo verrà in seguito raccolta e pubblicata in Id., Federalismo e libertà, Edizioni Fede!, Roma, 1925. A tal proposito vedi anche L. di Lembo, Il federalismo libertario e anarchico in Italia. Dal Risorgimento alla Seconda Guerra Mondiale, Edizioni Sempre Avanti!, Livorno, 1994, pp. 35-37.

${ }^{319} \mathrm{Id}$., Il posto degli anarchici nell'attuale crisi italiana, «Pensiero e Volontà», I, 1 novembre 1924. 
delle astrazioni, delle formule indefinite, della metafisica e del miracolismo, per basarci su di un metodo di lotta e di conquista positivo, comprensibile agli umili, rispondente alle necessità ed alla realtà dei tempi ${ }^{320}$.

Berneri ha iniziato a lasciare un segno, ma non partecipa direttamente in questo momento al dibattito sulla rivoluzione e il ruolo che gli anarchici dovrebbero giocarvi. In compenso il lodigiano non rinuncia a rilevare quali siano le insufficienze e i punti deboli del movimento, come appare evidente dalla sua Risposta ad una consultazione sui compiti immediati e futuri dell'anarchismo pubblicata nella sezione italiana del mensile parigino «La Revue Internationale Anarchiste» all'inizio del $1925^{321}$. In questo scritto Berneri sottolinea che

uno dei segni più tipici e più gravi dell'impreparazione degli anarchici ad affrontare $\mathrm{i}$ mille e vari problemi che la realtà presenta, mi pare questo cosmopolitismo della nostra propaganda; cosmopolitismo che non consiste nel carattere internazionale delle nostre iniziative, bensì nel prevalere della propaganda generica, a base prevalentemente dottrinaria, che non è sempre collegata con la particolare situazione politica e sociale della nazione $[\ldots]^{322}$.

Riemergono fortemente nell'anarchico lodigiano i motivi del concretismo e del problemismo, insomma quell'impostazione intellettuale che egli aveva mutuato da Salvemini e che stava riproponendo all'interno del movimento.

Prosegue Berneri:

Bisognerebbe che i compagni italiani si ambientassero anche politicamente, cercando di parlare la lingua del paese nel quale vivono, di partecipare alla vita sindacale del luogo, di esaminare i problemi sociali sotto gli aspetti particolari che quelli presentano in quel dato paese, per quella data categoria di lavoratori. Molti compagni cadono nell'errore di considerare il movimento, solo come una scuola di propaganda nella quale si ripetono i principî, e non come un'officina di indagini e di esperienze, rivolte alla vita più vasta della specifica attività politica. [...] ${ }_{E}$ necessaria una revisione di metodo. Bisogna che $i$ compagni più intelligenti e più colti trattino, utilizzando le particolari conoscenze ed esperienze, dei problemi tecnici della ricostruzione sociale, del movimento operaio, e di tutte le altre questioni che hanno importanza per la maggioranza degli uomini. Bisogna che tutti i compagni considerino il proprio lavoro (l'operaio la fabbrica, l'impiegato l'ufficio, l'insegnante la scuola, ecc.) come un fecondo campo di osservazione e di riflessione $[\ldots]^{323}$.

${ }^{320} \mathrm{C}$. Molaschi, Il posto degli anarchici nell'attuale crisi italiana cit.

${ }^{321} \mathrm{C}$. Berneri, Risposta ad una consultazione sui compiti immediati e futuri dell'anarchismo, «La Revue Internationale Anarchiste», I, 15 gennaio 1925, riprodotto in Id., Pietrogrado 1917 Barcellona 1937 cit., pp. 69-71, e in Id., Scritti scelti cit., pp. 95-97.

${ }^{322} \mathrm{Ibid}$.

${ }^{323}$ Ibid. I corsivi sono nostri. 
Berneri darà, sempre nel 1925, un contributo indiretto alla risvegliata riflessione sul federalismo all'interno del movimento scrivendo uno studio sul pensiero di Pëtr Kropotkin, uno dei «Maestri» dell'anarchismo, oltreché una delle sue principiali fonti di studio. La relazione tra $i$ due, come emergerà, è più complessa di quanto si potrebbe ritenere: se da un lato la riflessione "scientifica" kropotkiniana sulla costruzione spontanea della società di liberi e uguali è senza dubbio un bersaglio della critica berneriana, dall'altro il russo è riconosciuto da Berneri come uno dei più compiuti teorici del federalismo libertario, tema che è ora tra i più cari al lodigiano.

Lo studio viene pubblicato inizialmente in forma di articolo a puntate su «Fede!», tra febbraio e marzo 1925, col titolo Un federalista russo. Pietro Kropotkin, e in seguito viene rivisto, ampliato e nuovamente pubblicato come opuscolo sempre dalla casa editrice della rivista «Fede!» ${ }^{324}$. A tal proposito, ritornando sull'ipotesi di retrodatazione avanzata per Il valore civile del federalismo ${ }^{325}$, ci sembra di poter aggiungere ai nostri argomenti anche la mancanza in tale frammento di riferimenti a questo studio sul federalismo libertario di Kropotkin: quest'ultimo, accettando la datazione fin qui proposta dagli studiosi, sarebbe infatti precedente a Il valore civile del federalismo, ma è difficile credere che Berneri abbia escluso da una simile riflessione qualsiasi riferimento al federalismo libertario e, nel caso specifico, al pensiero kropotkiniano.

L'approfondimento sulla concezione federale di Kropotkin viene presentato da Berneri come uno studio su un federalismo libertario non innovativo rispetto a quello di Proudhon o di Bakunin, anche se il lodigiano ritiene vi sarebbero comunque degli elementi di grande interesse. Egli passa dunque a un excursus biografico dell'anarchico russo, soffermandosi in particolare su alcuni momenti che riteniamo significativi, ancora una volta, per cogliere un ulteriore sviluppo di quell'originale approccio concretista sviluppato da Berneri: egli, nell'analizzare e interpretare il pensiero politico di Kropotkin, pare selezionare, citare e mettere in rilievo alcuni aspetti della sua biografia politica, aspetti che sembrano valorizzare tematiche e metodologie che il lodigiano ha derivato dall'influenza di Salvemini e reinterpretato in chiave anarchica e rivoluzionaria.

Ad esempio, parlando del periodo in cui l'anarchico russo ha servito come ufficiale cosacco, viene evidenziato che egli

comincia a studiare vari progetti di amministrazione municipale, ma ben presto vede tutti gli sforzi di rinnovamento intralciati dai capi distretto, protetti dai Governatori generali, subenti, alla lor volta, gli ordini e le influenze del governo centrale ${ }^{326}$.

${ }^{324}$ C. Berneri, Un federalista russo. Pietro Kropotkin, Edizioni Fede!, Roma, 1925. L'opuscolo, che rispetto alla serie di articoli di «Fede!» presentava alcune aggiunte e integrazioni, è stato riprodotto in Id., Pietrogrado 1917 Barcellona 1937 cit., pp. 72-94, in Id., Il federalismo libertario cit., pp. 70-91, in Id., Anarchia e società aperta cit., pp. 258271, e in Id., Scritti scelti cit., pp. 235-255.

${ }^{325}$ Vedi supra.

${ }^{326}$ C. Berneri, Un federalista russo. Pietro Kropotkin, in Id., Pietrogrado 1917 Barcellona 1937 cit., p. 73 . I corsivi sono originali. 
La ricostruzione di Berneri si sofferma su un tema a lui caro, ossia la presa di coscienza da parte di Kropotkin che l'accentramento politico interviene a soffocare o a ostacolare ogni principio di vita o progetto autonomo. L'anarchico russo non ne attribuisce la colpa alla «mala voglia degli amministratori» quanto all'«amministrazione centralizzata e gerarchica»: in altre parole è la struttura governativa che è ritenuta inadeguata e opprimente. Questo passo avvicina le osservazioni del russo a quelle berneriane, poiché anche qui la critica allo Stato verte sul suo essere un ente centralizzato e male organizzato per via delle troppo numerose attribuzioni che si trova a dover svolgere.

In parallelo, secondo la ricostruzione del Berneri, Kropotkin inizia a osservare il funzionamento della «libera intesa fra gli interessati» che contribuirà alla sua formazione in senso anarchico, aspetto su cui Berneri avrà modo di soffermarsi in quello stesso anno, quando attenderà alla traduzione e alla prefazione della prima edizione italiana del kropotkiniano Il mutuo appoggio (1902) ${ }^{327}$. Tornando alla trattazione berneriana, Kropotkin aveva provato a stabilire contatti coi gruppi di sinistra che operano per la rigenerazione della Russia attraverso gli zemstvos (consigli di distretto o di provincia), ma ogni operazione

era sospettata come separatista, come tendente a creare uno Stato dentro lo Stato, e perseguitata a tal punto che qualsiasi tentativo di miglioramento nel campo amministrativo, sanitario e scolastico abortiva miseramente, portando con sé la rovina di interi gruppi degli eletti agli zemstvos ${ }^{328}$.

Ci sembra importante sottolineare come Berneri insista sul fatto che l'operazione di Kropotkin non sia stata meramente teorica e di principio, ma anzi tesa a rendere attuabili nell'immediato le proprie idee libertarie attraverso un impegno diretto nelle amministrazioni locali. Anche se tali tentativi risultano essere costantemente frustrati dal governo centrale, il tentativo di porre la progettualità libertaria su un piano costruttivo superando le pregiudiziali antistatali deve senza dubbio essere interessante per Berneri, che decide pertanto di porla in primo piano.

Lo scritto berneriano riferisce che tra il 1879 e il 1882 Kropotkin pubblica diversi articoli sul «Révolté» di Ginevra che confermano la sua critica antistatale e il suo radicarsi su posizioni federaliste e anarchiche, ponendo l'accento su una serie di contenuti che, di nuovo, ritornano su temi già agitati dal lodigiano sulla stampa anarchica:

Ovunque vi è l'accentramento [egli] trova potente la burocrazia [...]. E l'accentramento, portante al funzionarismo ad oltranza, appare al Kropotkin come una delle caratteristiche del regime rappresentativo. Egli vede nel parlamentarismo il trionfo dell'incompetenza, e così parla, con pittoresca ironia, dell'attività

${ }^{327}$ Vedi P. Kropotkin, Il mutuo appoggio: un fattore dell'evoluzione, Casa Editrice Sociale, Milano, 1925.

${ }^{328}$ C. Berneri, Un federalista russo. Pietro Kropotkin cit., p. 75 . Il corsivo è originale. 
amministrativa e legislativa del deputato, che non è chiamato a giudicare e a provvedere per quanto è di sua competenza e si riferisce al proprio collegio ma ad emettere un'opinione, a dare un voto sopra una serie variante all'infinito delle questioni che sorgono in quella mastodontica macchina che è lo Stato centralizzato $^{329}$.

Kropotkin ha parallelamente osservato anche la realtà delle libere associazioni, tema libertario di lungo periodo che Berneri sottolinea quale elemento di valore cruciale all'interno di un paradigma anarchico e federale: un anarchismo che voglia mettere in pratica le sue idee accettando il compromesso con la realtà non deve per questo perdere di vista gli elementi costitutivi del proprio pensiero.

Il lodigiano sta cercando, appare più che mai chiaro, di far emergere tutti quegli aspetti della vicenda intellettuale e politica di Kropotkin che sembrerebbero confermare la bontà del concretismo rivoluzionario che egli ha sviluppato a partire dalla lezione salveminiana. Non è un caso dunque che lo stesso Berneri ponga in rilievo che l'anarchico russo si sia appassionato a studi storici quando si trasferisce in Inghilterra, dedicandosi ad approfondire e comprendere meglio appunto lo sviluppo storico dello Stato. Kropotkin ne avrebbe tratto come conclusione che l'eccessivo accentramento aveva causato il tracollo dell'impero romano, favorendo in seguito la progressiva rinascita comunale. In merito alla Rivoluzione francese, l'anarchico russo si dirà invece avversario della

\begin{abstract}
borghesia dell'89 sognante «l'abolizione di tutti i poteri locali e parziali che costituivano altrettante unità autonome nello Stato, l'accentramento di tutta la potenza governativa nelle mani di un potere esecutivo centrale, strettamente sorvegliato dal Parlamento - strettamente obbedito nello Stato e conglobante tutto: imposte, tribunali, polizia, forze militari, scuole, sorveglianza poliziesca, direzione generale del commercio e dell'industria - tutto ${ }^{330}$.
\end{abstract}

La conclusione che ne trae Berneri è che

L'epoca dei Comuni e della Rivoluzione francese furono, come per il Salvemini, i due campi storici in cui il Kropotkin trovò conferme alle proprie idee federaliste ed elementi di sviluppo della sua concezione libertaria della vita e della politica ${ }^{331}$.

Ecco emergere di nuovo e con evidente forza la presenza e l'influenza di Salvemini. Berneri coglie e stabilisce un originale parallelo nel rilevare questa similitudine di interessi di indagine storica tra il suo maestro e l'anarchico russo: questa convergenza lo porta ad avvicinare e appaiare quelle che sono tra le maggiori influenze sulla sua formazione politica e intellettuale, non può dunque non convincersi che esista una sorta di continuità o quanto meno di contiguità tra le osservazioni dei due, continuità e affinità che a questo punto rafforzerebbero le sue

\footnotetext{
${ }^{329}$ Ivi, p. 76. Il corsivo è nostro.

${ }^{330}$ C. Berneri, Un federalista russo. Pietro Kropotkin cit., p. 79.

${ }^{331}$ Ivi, p. 80.
} 
posizioni e la sua ipotesi sulla possibilità e la necessità per il movimento anarchico di adottare un nuovo approccio più operativo e meno astratto, di aprirsi agli stimoli che provengono dall'approfondimento dei problemi e dalla ricerca di risposte concrete, di trovare punti di contatto e di intesa con altri movimenti e correnti politiche, soprattutto per quel che riguarda l'elaborazione di un progetto federale quale traduzione più immediata e operativa dei principi anarchici nel piano della realtà.

L'opinione sull'anarchico russo cambia in parte non appena il lodigiano si concentra sugli studi di Kropotkin dedicati alle teorie socialiste. Viene rilevata la sua critica ai cosiddetti utopisti come anche al collettivismo di stato, tuttavia il suo appellarsi allo «spirito collettivo» appare al Berneri troppo vago e generico:

È una specie di divinità, della quale Saverio Merlino ha scritto [...] che fa la parte del coro nelle tragedie greche, e che i più acuti rappresentanti dell'anarchismo sono lontani dall'adorare. Se il federalismo kropotkiniano pecca di indeterminatezza e di eccessiva fiducia nelle capacità politiche del popolo, si rende notevole per la larghezza di vedute ${ }^{332}$.

Kropotkin non è dunque immune all'appellarsi a formule e principi astratti, tuttavia il lodigiano torna ad apprezzarne la prospettiva quando ad esempio l'anarchico russo afferma che le parole «'Federalismo e autonomia non bastano. Non sono che parole per scoprire l'autorità dello Stato accentrato'» ${ }^{333}$, una dichiarazione che abbiamo visto essere condivisa e più volte ribadita da Berneri. La forma politica ideale è dunque quella del «Comune libero», rivoluzionario in politica quanto nelle questioni di produzione e scambio:

O la Comune sarà assolutamente «libera di darsi tutte le istituzioni che vorrà e di fare tutte le riforme e rivoluzioni che riterrà necessarie», o resterà «una semplice succursale dello Stato, inceppata in tutti i suoi movimenti, sempre sul punto di entrare in conflitto con lo Stato e certa di essere vinta nella lotta che ne avrebbe». Per il Nostro dunque i comuni liberi sono l'ambiente necessario alla rivoluzione perché essa raggiunga il suo massimo sviluppo ${ }^{334}$.

Berneri evidenzia al contempo che Kropotkin abbia però stimolato la ricerca di nuove soluzioni operative da parte di chi intenda trasformare l'organizzazione sociale in senso libertario ed egualitario, come rileverebbe un passaggio del suo famoso La scienza moderna e l'Anarchia (1901) in cui egli sostiene che

l'uomo sarà costretto a trovare nuove forme di organizzazione per le funzioni sociali che lo Stato esplica attraverso la burocrazia e che «finché questo non si farà, nulla

\footnotetext{
${ }^{332}$ C. Berneri, Un federalista russo. Pietro Kropotkin cit. p. 81.

${ }^{333} \mathrm{Ibid}$. Il corsivo è originale.

${ }^{334}$ Ivi, p. 82.
} 
sarà fatto», ma non poté, per la sua vita ora avventurosa, ora strettamente scientifica, sviluppare sistematicamente la sua concezione federalista ${ }^{335}$.

La citazione virgolettata è con ogni probabilità tratta dalla parte finale del capitolo che l'anarchico russo aveva dedicato alle imposte, ritenute corollario esemplare dell'accentramento politico e dell'ipertrofia statale:

E finché lo Stato, armato dell'imposta, continuerà ad esistere, l'emancipazione del proletariato non potrà compiersi in alcun modo: né per la via delle riforme e neppure con la rivoluzione. Perché se la rivoluzione non schiaccia questa piovra, se non taglia le sue teste ed i suoi tentacoli, sarà strangolata dalla mala bestia ${ }^{336}$.

Più in generale, Berneri sta però riassumendo le critiche rivolte da Kropotkin allo Stato moderno nelle ultime pagine de La scienza moderna e l'anarchia, in cui egli sostiene:

Per emanciparsi, è assolutamente indispensabile alle masse che tutto producono, senza essere ammesse a regolare il consumo della loro produzione, di trovare i mezzi che permettano loro di sviluppare le proprie forze creatrici e d'elaborare esse stesse le forme nuove egualitarie, del consumo e della produzione.

[...] Ed è la stessa cosa per le forme d'organizzazione politica. Per liberarsi dallo sfruttamento che subiscono sotto la tutela dello Stato, le masse non possono tollerare il dominio di forme, che impediscono il fiorire della vita popolare, perché furono elaborate dai governi appunto per perpetuare la servitù del popolo [...]. Necessita trovare delle nuove forme per servire allo scopo contrario ${ }^{337}$.

L'attenzione alla complessità dell'obiettivo da realizzare e l'appello dunque a farsi carico di questo problema in maniera attenta, al di là dei principi e delle pulsioni insurrezionali, uniscono ancor di più il russo e il lodigiano. Nel guardare alla biografia di Kropotkin, Berneri analizza anche il suo «interventismo democratico» al momento della Grande Guerra, un altro aspetto che avvicina la vicenda intellettuale e politica dell'anarchico russo a quella di Salvemini, un altro elemento che non ci pare casuale che l'anarchico lodigiano faccia emergere nella sua ricostruzione; un elemento che, anche se ritenuto contraddittorio, rileva tuttavia una sua certa coerenza - comprensibile, ancorché non accettabile dal punto di vista berneriano - poiché Kropotkin «era contro la Germania perché vedeva in essa un pericolo per l'autonomia dei popoli e per il decentramento» ${ }^{338}$. La speranza del russo, secondo Berneri, è anche che la guerra ponga fine all'autocrazia zarista, favorendo il suo trasformarsi in senso rivoluzionario e federale, per il quale la Russia è ritenuta ormai matura.

\footnotetext{
${ }^{335}$ Ibid.

${ }^{336}$ P. Kropotkin, Sovremennaia nauka i anarkhizm, Russian Free Press, London, 1901, tr. it. Casa Editrice Sociale, Milano, 1922, p. 181.

${ }^{337}$ Ivi, p. 244.

${ }^{338}$ C. Berneri, Un federalista russo. Pietro Kropotkin cit., p. 85.
} 
Viene ovviamente posto in rilievo che Kropotkin sarà un acceso critico dei caratteri centralisti assunti dalla rivoluzione bolscevica:

In un'intervista ad Augusto Suochy, pubblicata dal Er Keuntis Befreiung di Vienna, il Kropotkin dice: «Noi dovremmo aver dei Consigli di comune. I Consigli comunali dovrebbero lavorare di propria iniziativa. Provvedere, ad esempio che, in caso di cattiva raccolta, la popolazione non manchi dei generi di prima necessità. Il governo centralizzato è, in questo caso, un apparato oltremodo pesante. Mentre federalizzando i Consigli si creerebbe un centro vitale». [...] In una delle sue ultime lettere (23 dicembre 1920) all'anarchico olandese De Reyger, che fu pubblicata su Vrije Socialist, il nostro scriveva: «La Rivoluzione sociale ha preso disgraziatamente, in Russia, un carattere centralizzato e autoritario» ${ }^{339}$.

La critica al bolscevismo è intransigente, nonostante Kropotkin riconosca che con la Rivoluzione sono state introdotte nella vita russa nuove concezioni sulla funzione sociale e sui diritti del lavoro, oltre che sui doveri dei singoli. La critica dell'anarchico russo è rivolta alla dittatura di partito e alle basi fortemente centralizzate della repubblica comunista, confidando per contro sul fatto che l'esperienza dei soviet o dei consigli potesse essere una valida alternativa a tale modello, altra posizione che l'anarchico lodigiano come abbiamo visto sostiene - e che, come vedremo, non cesserà mai di sostenere. Berneri afferma che Kropotkin non espose mai apertamente queste idee per timore di favorire i nemici del socialismo e trovò attriti anche con gli anarchici russi privi di uno spirito costruttore, finendo con l'individuare nel sindacato l'ultima possibilità reale per una rivoluzione autonomista e antiautoritaria.

Il passaggio conclusivo dello studio di Berneri rivela quelli che, secondo l'autore, sono i difetti della rappresentazione kropotkiniana così come il suo principale pregio:

Nel federalismo kropotkiniano vi è un eccessivo ottimismo, vi sono semplicismi e contraddizioni, ma vi è una grande e feconda verità: che la libertà è condizione di vita e di sviluppo per i popoli; che soltanto quando si governa da sé e per sé [si] è al sicuro dalla tirannide e certo del suo proseguire ${ }^{340}$.

È possibile notare come tutto lo studio sul pensiero federalista di Kropotkin ripercorra di fatto i diversi temi e interessi affrontati da Berneri nel corso della sua formazione politica, dagli studi sulla burocrazia e la centralizzazione a quelli sulla Rivoluzione russa e i suoi esiti, passando per gli studi storici e l'elaborazione di una teoria federalista e libertaria. Il saggio berneriano non è insomma un semplice esercizio di erudizione o una sintesi divulgativa sul pensiero e l'azione dell'anarchico russo, ma presenta anzi tutti gli aspetti di una rilettura atta a insistere su quanto il lodigiano avesse fino a quel momento agitato e sostenuto all'interno del suo stesso movimento. Berneri non solo legge e ritrova parte di se stesso e delle sue idee in

${ }^{339} \mathrm{C}$. Berneri, Un federalista russo. Pietro Kropotkin cit., p. 87. I corsivi sono originali.

${ }^{340}$ Ivi, p. 94. 
Kropotkin, ma si confronta allo stesso tempo con il pensiero del «Maestro» e a partire da questo insiste sui principali aspetti caratteristici della sua visione politica.

$\grave{E}$ forse in questo momento, ci sembra, che il federalismo libertario arriva a rappresentare ai suoi occhi la sintesi organica di tutte le sue aspirazioni, di tutti i suoi scritti, di tutto il suo impegno: muoversi entro i fecondi principi e intuizioni della tradizione anarchica, ma fuori allo stesso tempo dalle vaghezze teoriche e pratiche. Il principio di libertà e l'autogoverno restano le coordinate principali per il movimento anarchico, ma non devono restare sulla carta della propaganda e devono invece farsi concrete in un "programma minimo" federalista.

\section{La libertà possibile: un programma concreto contro le astrattezze}

La necessità di un programma concreto è certo fortemente sentita da Berneri anche in ragione dell'affermarsi del regime fascista in Italia. Corrono tempi difficili e l'anarchico lodigiano non vuole certo restare a guardare: tra 1923 e 1926 egli si sta spostando tra diverse scuole in qualità di insegnante, passando da incarichi annuali prima a Montepulciano, quindi a Cortona e infine a Camerino; non rinuncia comunque a frequenti soggiorni a Firenze per mantenere i contatti tanto col movimento quanto con altri gruppi antifascisti. Collabora tra l'altro con il primo movimento antifascista clandestino sorto nella penisola, l'«Italia Libera», ed Ernesto Rossi lo ricorda con affetto insieme a un elenco di aderenti che vale la pena citare integralmente per restituire lo spessore intellettuale, oltreché morale, del fenomeno:

Dino Vannucci, Carlo Rosselli, Nello Rosselli, Enrico Bocci, Gaetano Pilati, Piero Gobetti, Camillo Berneri, Umberto Ceva, Eugenio Colorni, Leone Ginzburg, Mario Damiani, Giannantonio Manci, Gigino Battisti, tutti oggi scomparsi, e i più per mano fascista...Quale significato avrebbe la mia vita, e come potrei ancora avere fiducia negli uomini, se non li avessi, in un certo momento, incontrati sulla mia stessa $\operatorname{strada}^{341}$ ?

Anche la madre di Berneri, rievocando quegli anni, rammenta di una sera in cui vide suo figlio allegro «mentre montava sulla automobile del povero Rosselli per andare con lui e due altri a diffondere dei manifesti» ${ }^{342}$; è assai probabile che si tratti di una delle affissioni clandestine organizzate e messe in atto in quel periodo dall'«Italia Libera» che, come ricorda Ernesto Rossi, poteva avvalersi in queste operazioni «della macchina di Nello Rosselli - la "Bianchina" - da lui stesso guidata» ${ }^{343}$.

Il suo maestro Salvemini lo ricorda invece tra coloro che contribuivano alla difficile e rischiosa distribuzione fuori dalla Toscana del foglio clandestino «Non

\footnotetext{
${ }^{341}$ E. Rossi, L'«Italia Libera», in Non Mollare (1925) cit., pp. 50-51. Si veda anche, più in generale, L. Zani, Italia Libera. Il primo movimento antifascista clandestino (1923-1925), Laterza, Roma-Bari, 1975.

${ }^{342}$ A. Fochi, Con te, figlio mio! cit., p. 111.

${ }^{343}$ E. Rossi, L'«Italia Libera» cit., p. 55.
} 
Mollare», da lui fondato insieme ai fratelli Rosselli, Ernesto Rossi, Nello Tarquandi e Dino Vannucci:

I pacchetti [contenenti gli esemplari del foglio da distribuire] erano portati a mano ai destinatari fuori Firenze da ferrovieri viaggianti o ambulantisti postali. Varie centinaia di copie andavano per posta, ma erano imbucate in città diverse. [...]

Fuori di Firenze aiutavano Camillo Berneri, che insegnava in una scuola normale dell'Umbria; Riccardo Bauer, Ferruccio Parri e Vittorio Albasini Scrostati a Milano; Egidio Meneghetti a Padova; Max Ascoli, Tullio Ascarelli, Umberto Morra di Lavriano, Lucangelo Bracci e Umberto Zanotti-Bianco a Roma ${ }^{344}$.

Il forte legame col professore pugliese è altresì testimoniato da un altro ricordo della madre di Berneri che ricorda che, saputo della possibilità di un attentato contro il Salvemini, Camillo «si recò fino all'abitazione del professore per avvertirlo di non farsi vedere fuori»» ${ }^{345}$.

La situazione si farà però sempre più insostenibile per Berneri che, oltre a essere ben noto alla polizia, è incapace di nascondere la propria opposizione al regime e di evitare pertanto di attirare l'attenzione su di sé. Una testimonianza del suo atteggiamento di opposizione ci viene proprio dalle carte del Casellario Politico Centrale che ci raccontano di un episodio avvenuto nel 1926 a Camerino quando, in occasione di una visita del re Vittorio Emanuele III a Macerata, egli si palesa antifascista di fronte ai suoi studenti: un'informazione riservata manoscritta di Vittorio Moneta, dirigente del fascio locale, riporta che «agli alunni che gli chiesero vacanza per recarsi al fascio di Macerata a rendere omaggio al sovrano, rispose con parole offensive sia a S. M. che al Regime» ${ }^{346}$; Moneta ha iniziato da allora a sorvegliare Berneri; non è certo il solo, anzi leggendo le annotazioni autobiografiche dell'anarchico lodigiano è possibile avere un'idea di quanto l'aria si stesse facendo asfissiante per lui, tanto da portarlo infine ad optare per l'esilio volontario:

Le ragioni dell'espatrio furono le seguenti: $1^{\circ}$ un'aggressione subita da parte dei fascisti di Camerino [...] mi aveva data l'impressione di un completo isolamento; $2^{\circ}$ la sorveglianza della polizia si faceva così stretta che ogni mio passo era segnalato e seguito; $3^{\circ}$ la non-iscrizione alla corporazione, la decisione presa di non giurare, il veto prefettizio ad una nomina a cui avevo pieno diritto essendo primo fra $\mathrm{i}$ concorrenti, le continue complicazioni disciplinari create dal mio atteggiamento interamente refrattario alle nuove disposizioni (cerimonie, saluto romano, ecc) ${ }^{347}$.

\footnotetext{
${ }^{344}$ G. Salvemini, Il «Non Mollare», in Non Mollare (1925) cit., p. 7. Berneri tuttavia insegnava allora a Camerino, è probabile che il Salvemini si sia confuso per via della prossimità geografica.

${ }^{345}$ A. Fochi, Con te, figlio mio! cit., p. 126.

${ }^{346}$ Informazione riservata manoscritta di Vittorio Moneta alla Prefettura di Macerata, 1 maggio 1928, in ACS, Min. In., CPC, Berneri Camillo, b. 537, fsc. I.

${ }^{347} \mathrm{C}$. Berneri, Nota autobiografica, in AFB, fondo C. Berneri, cass. I
} 
Dopo aver analizzato la sua ampia e varia produzione sulla stampa, è adesso necessario soffermare la nostra attenzione su due scritti rimasti a loro tempo inediti, che abbiamo potuto consultare presso l'Archivio Famiglia Berneri-Aurelio Chessa: questi manoscritti contribuiscono a completare la ricostruzione dello sviluppo raggiunto dal pensiero federalista di Camillo Berneri nella prima metà degli anni '20 e a restituirlo in tutta la sua ampiezza.

Il primo, significativamente intitolato La concezione anarchica dello Stato $^{348}$, viene con ogni probabilità steso all'inizio del 1926 ed è stato pubblicato per la prima volta solo nel 2001 nell'antologia curata da Pietro Adamo ${ }^{349}$. Già dal titolo è possibile evincere facilmente il motivo per il quale Berneri abbia deciso di non diffonderlo: nessun anarchico può accettare di avere una concezione particolare dello Stato, a meno che non sia quella di contrapporvisi frontalmente e di lottare per la sua demolizione. L'anarchico lodigiano, lo abbiamo visto, ha invece sviluppato nel tempo un'idea differente e in questo scritto tenta di tirare le somme di quella che egli ritiene dovrebbe essere la natura della critica anarchica allo Stato.

Scrive Berneri:

Vi sono degli anarchici che lo negano [lo Stato]: gli individualisti, specie gli stirneriani. Ma gli anarchici comunisti quando negano lo Stato lo confondono col governo; o intendono negare lo Stato accentrato, organo di conservazione politica e sociale; cioè lo Stato nella sua attuale fase di sviluppo.

Questo sovrapporsi del termine Stato indicante lo Stato nella sua forma politica (Monarchia, Repubblica) e nella sua funzione sociale (borghese, comunista) al termine Stato indicante quel complesso di organi direttivi, tecnici ed amministrativi, che costituisce per la società quello che gli organi vitali e il sistema osseo costituiscono per l'uomo, è necessario, ma è [...] fonte di infinite prevenzioni contro il nostro programma e contro il nostro movimento ${ }^{350}$.

È necessario porre in evidenza come torni a riproporsi la distinzione che Berneri aveva già proposto nel dibattito sul federalismo su «Pagine Libertarie» tra Stato in quanto organismo politico e Stato in quanto organismo amministrativo ${ }^{351}$, segno del coerente proseguire della sua riflessione su questo tema.

Proseguendo, l'anarchico lodigiano precisa la sua posizione

\footnotetext{
${ }^{348}$ Id., "La concezione anarchica dello Stato", in AFB, fondo C. Berneri, cass. IV, Opere di carattere politico, $\mathrm{n}^{\circ} 1$, Anarchia e anarchismo. Il manoscritto è riprodotto in Id., Anarchia e società aperta cit., pp. 124-128.

${ }^{349}$ La datazione, con la quale concordiamo, è stata proposta da Adamo nella nota introduttiva a C. Berneri, La concezione anarchica dello Stato, in Id., Anarchia e società aperta cit., p. 124; meno plausibile ci sembra quella di De Maria che lo ha invece collocato nei primi anni dell'esilio, vedi C. De Maria, Camillo Berneri cit., p. 25 (nota a piè di pagina).

${ }^{350} \mathrm{C}$. Berneri, "La concezione anarchica dello Stato", c. 1. Le sottolineature sono originali. La concezione anarchica dello Stato cit., p. 125.

${ }^{351}$ Vedi supra.
} 
La società, con tutte le sue istituzioni: familiari, economiche, religiose, politiche, ecc, non può identificarsi con lo Stato, che rimane, anche se ridotto a funzione di ente di pubblica utilità e necessità, un organismo specifico, che è passibile di sviluppo appunto perché non comprende tutta la società. Appunto perché lo Stato, come organo specifico, tende a diventare una società nella società, $[\ldots]$ crediamo che la società $[\ldots]$ debba rimanere, per funzione storica, in una posizione antitetica di fronte allo Stato ${ }^{352}$.

La chiave, dunque, è quella di garantire l'autonomia della società, articolata in varie forme di associazione, di fronte allo sviluppo delle tendenze accentratrici dello Stato - altro tema che ha caratterizzato gran parte della riflessione berneriana. La battaglia anarchica dovrebbe pertanto mettere da parte gli assoluti del proprio programma massimo e insistere piuttosto sui propri contenuti autonomisti, dandosi un programma minimo che proponga soluzioni immediate e praticabili in questo senso; per il lodigiano non sarebbe che una prima fase, da perseguire

\title{
fino a quando lo Stato si riduca ad essere la sintesi direttiva di comunità autonome e federate nelle quali il libero accordo degli interessati risolva l'antagonismo fra l'individuo e la società. Cioè fino a quando lo Stato si dilati nella sua sfera di formazione e si restringa nella sua sfera di azione, fino a identificarsi con la Società 353 .
}

In altre parole, Berneri suggerisce al movimento di considerare l'avvio di una battaglia autonomista di lungo corso, una battaglia che potrà portare in ultima istanza fino a un dissolversi dello Stato nella libera federazione storicamente teorizzata dagli anarchici. La sua "eresia", da un punto di vista anarchico, sta nel suo interpretare lo Stato come organismo amministrativo da tenere in piedi, seppure concependolo e organizzandolo diversamente, in quanto nello «Stato anarchico al: sono governati, si sostituisce il: si governano» ${ }^{354}$.

Con ogni probabilità qui si sta raggiungendo l'apice dell'eccentricità delle idee berneriane, e i passaggi successivi non fanno che accentuare tale tendenza:

\begin{abstract}
L'anarchico comunista vorrebbe la società costituita, economicamente e politicamente, da associazioni. E il termine associazione è da lui anteposto comunemente a quello di $\underline{\text { Stato. }}[\ldots]$

L'anarchismo nega lo Stato-governo, non nega lo Stato inteso come sistema di rappresentanze, di organi di collegamento e direttivi. L'Anarchia è la città ideale, lo Stato libertario è la sua approssimazione storica. L'Anarchia è religione, lo Stato
\end{abstract}

\footnotetext{
${ }^{352}$ C. Berneri, "La concezione anarchica dello Stato", cc. 1-2. La concezione anarchica dello Stato cit., p. 125.

${ }^{353}$ C. Berneri, "La concezione anarchica dello Stato", c. 2. La concezione anarchica dello Stato cit., p. 125. Il corsivo è nostro.

${ }^{354}$ Ivi, c. 3. La concezione anarchica dello Stato cit., p. 125.
} 
libertario è politica. Tra l'Anarchia e lo Stato libertario vi è la distanza che separa l'associazione dalla società ${ }^{355}$.

In altre parole, per giungere allo «Stato anarchico» - ossimoro che avrebbe fatto insorgere non pochi compagni di Berneri - dove tutta la società è in grado di governarsi da sola e senza bisogno di coazione, bisogna accettare di confrontarsi con la storia e con la politica, passando per lo «Stato libertario», un'organizzazione federale in cui le autonomie delle associazioni sottraggano autorità allo Stato e in cui, va da sé, bisognerà accettare qualche forma residua di autorità:

La legge $\mathrm{A}$, quella $\mathrm{B}$, quella $\mathrm{C}$, ecc. sono leggi assurde o avverse al popolo. Ciò non implica: tutte le leggi sono assurde e dannose. Assurdo e dannoso è che sia un Parlamento a promulgarle, che sia uniforme la loro applicazione, ecc. Ma vi sono leggi opportune. Quindi: dato che vi sono leggi utili, mentre si deve lavorare affinché gli uomini sappiano autodisciplinarsi senza coazione, l'ordine nuovo dovrà promulgare leggi utili alla cui applicazione tutti i cittadini dovranno cercare di portare il proprio contributo ${ }^{356}$.

È certo lo sviluppo di quella riflessione sul rapporto tra libertà e autorità sviluppata dal lodigiano due anni prima sulle colonne di «Fede!» ${ }^{357}$, che pare ora prendere corpo in alcune linee di programma federalista libertario:

Molti anarchici concepiscono l'ordine nuovo come una rete di associazioni. Che le associazioni culturali, di lavoro, sportive, ecc. si svilupperanno enormemente e che potranno avocare a sé molte funzioni che oggi sono privilegio dello Stato e dei Comuni è evidente. Che noi dobbiamo cercare di sviluppare al massimo l'associazione è altrettanto evidente. Ma è anche evidente che la società è qualche cosa di più vasto e di diverso dell'associazione ${ }^{358}$.

L'apice della maturazione delle idee berneriane sin qui osservate e analizzate è raggiunto però in altri due frammenti del 1926, scritti probabilmente a Parigi e costituenti però un documento unitario ${ }^{359}$ : sorvegliato strettamente dal regime fascista, Berneri si era infine risolto a lasciare clandestinamente l'Italia nell'aprile di

${ }^{355}$ C. Berneri, "La concezione anarchica dello Stato", c. 3 e prosieguo del documento numerato separatamente, c. 1. Le sottolineature sono originali. La concezione anarchica dello Stato cit., p. 126.

${ }^{356}$ Ivi, prosieguo del documento numerato separatamente, c. 3. La concezione anarchica dello Stato cit., p. 127.

${ }^{357}$ Vedi supra.

${ }^{358}$ C. Berneri, "La concezione anarchica dello Stato", prosieguo del documento numerato separatamente, c. 4. La concezione anarchica dello Stato cit., p. 128.

${ }^{359} \mathrm{C}$. Berneri, manoscritto senza titolo e manoscritto acefalo, in AFB, fondo C. Berneri, cass. IV, Opere di carattere politico, $\mathrm{n}^{\circ} 1$ cit. La datazione, con cui concordiamo, è stata annotata sulla prima pagina dalla moglie Giovanna Caleffi, vedi Camillo Berneri, Pietrogrado 1917 Barcellona 1937 cit., p. 95. 
quello stesso anno. Il rafforzamento del regime fascista e il susseguente espatrio in Francia lo portano ora ad accentuare le sue riserve sul movimento anarchico e a insistere sulla necessità di darsi una nuova e operativa linea programmatica.

Rimasto a suo tempo inedito e privo di titolazione, il manoscritto è stato pubblicato per la prima volta da Masini e Sorti nel 1964 con il titolo Per un programma d'azione comunalista ${ }^{360}$; ci pare che la scelta renda bene il carattere di questo testo che arriva, come abbiamo mostrato, a seguito di un percorso di analisi e di proposte chiare da parte di Berneri in seno al movimento anarchico. Al contrario, la titolazione Sul comunalismo, adottata successivamente nell'antologia di Pietro Adamo e riproposta da Carlo De Maria, ci pare che ne alteri il significato ${ }^{361}$ : più che un semplice testo sul tema del comunalismo, il frammento berneriano ci risulta solo apparentemente incompleto e mostra anzi - soprattutto leggendolo a completamento della sua produzione edita e inedita sin qui analizzata - tutti i caratteri di una chiara e inequivocabile esortazione affinché gli anarchici accettino di darsi un programma politico, capace di indicare mete reali e immediate, di rispondere a sentimenti concreti, vivi e comuni.

Berneri vi afferma:

Il problema della nostra tattica rivoluzionaria e post-rivoluzionaria è male basato e peggio sviluppato. Socialmente siamo imprigionati nel dualismo: proletariatoborghesia, mentre il proletariato tipico è minoranza ed è fiacco e disorientato e vi sono vari ceti intermedi, ben più importanti e combattivi. Non ne abbiamo tenuto conto, noi rivoluzionari, ed abbiamo avuto il fascismo [...].

Di paradisi comunisti se ne parlerà fra qualche secolo. Ora è roba da far ridere e far pietà insieme. L'anarchismo non ha, al di fuori di quello sindacale, che un terreno sul quale battersi proficuamente nella rivoluzione italiana: il Comunalismo. Terreno politico. Funzione liberale democratica. Scopo, la libertà dei singoli e la solidità degli enti amministrativi locali. Mezzo: l'agitazione su basi realistiche, con l'enunciazione di programmi minimi ${ }^{362}$.

Colto l'isolamento degli anarchici a seguito di una stagione in cui essi rappresentavano invece un importante punto di riferimento, Berneri insiste affinché la battaglia sociale del sindacalismo sia affiancata da quella politica del comunalismo. Quest'ultima ha per il lodigiano un fine liberale e democratico, ma è soprattutto importante riuscire a passare dall'affermazione di tale battaglia all'azione

\footnotetext{
${ }^{360} \mathrm{C}$. Berneri, Per un programma d'azione comunalista, in Id., Pietrogrado 1917 Barcellona 1937 cit., pp. 95-99. Probabilmente anche questo scritto non fu sviluppato e pubblicato in quanto Berneri ritenne che i tempi non fossero maturi e che l'eventuale diffusione sarebbe potuta risultare controproducente, inefficace al suo scopo. Il frammento è stato riprodotto anche in Id., Il federalismo libertario cit., pp. 47-50, e in Id., Scritti scelti cit., pp. 108-111, con la medesima titolazione proposta dalla raccolta del 1964.

${ }^{361}$ Vedi C. Berneri, Sul comunalismo, in Id., Anarchia e società aperta cit., pp. 129-132, e C. De Maria, Camillo Berneri cit., p. 17 (nota a piè di pagina).

${ }^{362} \mathrm{C}$. Berneri, manoscritto senza titolo, c. 3. La sottolineatura è originale. Per un programma d'azione comunalista, in Id., Pietrogrado 1917 Barcellona 1937 cit., pp. 96-97.
} 
su un piano pratico e concreto, reale, attraverso «programmi minimi». Non risulta chiaramente, ma alla luce di quanto abbiamo fin qui esposto, ci pare certa la possibilità di cogliere in questa impostazione berneriana la tendenza a cercare fin da subito lo scambio e l'eventuale alleanza con altre forze politiche - repubblicani di sinistra in primis - intorno a determinati punti programmatici quali appunto federalismo e autonomie. È dunque necessario che $\mathrm{i}$ principali rappresentati del federalismo anarchico vengano aggiornati ai tempi che verranno:

Ritornando a Proudhon, a Bakunin e a Pisacane, come fonti, ma aggiornando il loro pensiero al lume delle enormi esperienze di questi anni di delusioni e di sconfitte, potremo adattarlo alle situazioni sociali e politiche di domani, quali possiamo prevedere possibili se sapremo dare alla rivoluzione italiana un indirizzo autonomista, sul terreno sindacale e su quello comunale ${ }^{363}$.

Secondo Berneri, l'ottimismo dell'«evoluzionismo solidarista» e la negazione aprioristica di ogni forma di autorità hanno portato all'isolamento dell'anarchismo, in quanto refrattario allo studio meticoloso dei problemi e di conseguenza incapace di proporre una soluzione praticabile, di avvicinare l'idea alla realtà, l'astratto al concreto - insomma incapace, aggiungiamo noi, di porsi in quella prospettiva che il lodigiano aveva mutuato da Salvemini.

Berneri si lancia dunque in una chiara proposta di rinnovamento che ha tutti i caratteri di una dichiarazione programmatica:

Un anarchismo attualista, consapevole delle proprie forze di combattività e di costruzione e delle forze avverse, romantico col cuore e realista col cervello, pieno di entusiasmo e capace di temporeggiare, generoso e abile nel condizionare il proprio appoggio, capace, insomma di un'economia delle proprie forze: ecco il mio sogno. E spero di non essere solo ${ }^{364}$.

Se non si imbocca questa strada, Berneri intravede quale esito la desertificazione dell'anarchismo, il suo cristallizzarsi e dogmatizzarsi, in una parola l'approfondirsi ulteriore della sua crisi: «O la botte vecchia resisterà al vino nuovo, o il vino nuovo

${ }^{363}$ Ivi, c. 4. Per un programma d'azione comunalista, p. 97.

${ }^{364} \mathrm{C}$. Berneri, manoscritto acefalo, c. 7. La sottolineatura è originale. Per un programma d'azione comunalista, pp. 98-99. L'attualismo di cui parla Berneri ci appare sostanzialmente l'ennesima variazione sul tema della sua personale rielaborazione del concretismo di Salvemini: si oppone all'inattualità delle soluzioni storicamente avanzate dal movimento anarchico, tentando di portare la lotta su obiettivi e programmi quanto più possibile perseguibili e attuabili. Cfr. però su questa interpretazione anche S. D'Errico, Anarchismo e politica cit., pp. 253-262, G. Berti, Il pensiero anarchico cit., pp. 872-878, e G. Cerrito, L'anarchismo attualista di Camillo Berneri, in Atti del Convegno di studi su Camillo Berneri, Milano, 9 ottobre 1977, La Cooperativa Tipolitografica, Carrara, 1979, pp. 91-144. Non ci pare invece condivisibile un'influenza gentiliana nell'utilizzo di tale termine come sostenuto in C. De Maria, Una famiglia anarchica. La vita dei Berneri tra affetti, impegno ed esilio nell'Europa del Novecento, Viella, Roma, 2019, p. 204. 
cercherà una botte nuova $\rangle^{365}$. Con quest'ultima sentenza si chiude significativamente la fase di maturazione delle idee berneriane ed emerge con tutta evidenza come la sua posizione all'interno del movimento anarchico si stia facendo estremamente critica e tesa alla ricerca di una rivisitazione che non sminuisca il portato della tradizione politica libertaria, ma che possa, allo stesso tempo, aggiornarla e renderla operativamente efficace.

${ }^{365}$ C. Berneri, manoscritto acefalo, c. 7. Per un programma d'azione comunalista, p. 99. 



\section{Capitolo 3 \\ L'esilio senza requie e la costituente libertaria}

\section{1. «L'anarchico più espulso d'Europa»: l'elaborazione politica}

Perseguitato dal fascismo, Berneri ha infine deciso di lasciare l'Italia:

Nel 1926 decisi di emigrare, clandestinamente poiché la sorveglianza accanita della polizia, i precedenti arresti, un'istruttoria sospesa ma non chiusa, ecc. mi facevano pensare impossibile l'ottenere il passaporto. Passai la frontiera a Ventimiglia, nell'aprile, coadiuvato da due compagni. Dopo quattro mesi passavano il confine clandestinamente mia moglie e le mie due bambine ${ }^{366}$.

La prefettura di Macerata notificava l'avvenuto espatrio solamente il 30 aprile $^{367}$. Berneri si sistema inizialmente a Nizza, ma già il mese successivo raggiunge Parigi dove riprenderà contatti diretti col movimento anarchico italiano e internazionale. Il periodo da fuoruscito sarà per lui, come per tutti gli esuli politici italiani antifascisti, intensamente travagliato, ma le cose si faranno nel suo caso particolarmente difficili fin da subito: già osservato speciale della polizia francese, il lodigiano finisce col cadere nella rete di provocazione dell'OVRA e nel novembre 1928 comincia il suo «esilio senza requie» ${ }^{368}$, una serie rocambolesca di espulsioni, arresti, incarcerazioni, proroghe e sospensioni del permesso di soggiorno che durerà fino al luglio 1931 e

${ }^{366} \mathrm{C}$. Berneri, Nota autobiografica, in AFB, fondo C. Berneri, cass. I, $\mathrm{n}^{\circ} 1$, Carte personali.

${ }^{367}$ Nota della Prefettura di Macerata, 30 aprile 1926, in ACS, Min. In., CPC, Berneri Camillo, b. 537 , fsc. I.

${ }^{368}$ C. Berneri, Pensieri e battaglie, Comitato Camillo Berneri, Parigi, 1938, pp. 63-98. Berneri aveva anche intenzione di scrivere un libro sull'esilio, tanto l'esperienza lo aveva segnato. Vedi a questo proposito S. D'Errico, Anarchismo e politica. Nel problemismo e nella critica all'anarchismo del ventesimo secolo, il "programma minimo" dei libertari del terzo millennio. Rilettura antologia e biografica di Camillo Berneri, Mimesis, Milano, 2007, pp. 539-547. 
che lo costringerà a spostarsi in Belgio, Svizzera, Olanda, Lussemburgo e Germania con tre rientri clandestini in Francia ${ }^{369}$.

La vicenda è valsa a Berneri l'infelice e non certo invidiabile fama de «l'anarchico più espulso d'Europa» ${ }^{370}$ e lo spettro e la concretezza della prigione e dell'espulsione aleggeranno su di lui fino al luglio 1936, quando lascerà il territorio francese per portare la sua azione e il suo pensiero a misurarsi con l'esperienza della Guerra Civile spagnola.

\section{Riprendendo il filo del discorso}

Se è vero, come ha sostenuto Santi Fedele, che l'esperienza del fuoruscitismo sia stata non soltanto un'importante testimonianza etico-politica, ma anche e soprattutto un «travaglio politico e ideale» manifestatosi «nello sforzo costante da più parti operato per approfondire analisi, superare impostazioni ideologiche anacronistiche, svecchiare programmi ed elaborare strategie di lotta» in cui «tutto è sottoposto a critica, rivisto, aggiornato, rielaborato al confronto con realtà diverse e nel fuoco della battaglia antifascista» ${ }^{371}$, si può dire che per Berneri ciò non sia stato altro che riprendere il filo di un lungo discorso che - come abbiamo mostrato - ha radici lontane e che egli aveva svolto con una sorprendente continuità dal primo dopoguerra fino all'avvento del fascismo. Se insomma per il fuoruscitismo antifascista il periodo dell'esilio coinciderà con l'avvio di un lungo processo generalizzato di analisi autocritiche e di rivisitazione dei programmi, per Berneri ciò non costituirà niente di nuovo, ma solo il proseguire di una tensione e di una battaglia già da lungo tempo aperta.

L'anarchico lodigiano non ha fatto in tempo a varcare la frontiera tra Italia e Francia che già si è rimesso al lavoro per contribuire alla stampa anarchica e per riportare all'attenzione di compagni e militanti i temi a lui cari e le idee che va maturando. Interessante a questo proposito è l'analisi di un articolo su cui nessuno studioso o storico del pensiero berneriano si è mai finora sufficientemente soffermato, vale a dire La Comune di Parigi e l'idea federalista che compare il 27 aprile 1926 su «Culmine», quindicinale di Buenos Aires ${ }^{372}$. Si tratta di uno scritto

${ }^{369}$ Per la ricostruzione di questa travagliata vicenda si veda C. De Maria, Camillo Berneri. Tra anarchismo e liberalismo, Franco Angeli, Milano, 2004, pp. 36-75.

${ }^{370}$ Tale epiteto, che ha avuto larga fortuna, si deve al giornalista Emiliani, vedi V. Emiliani, Gli anarchici. Vite di Cafiero, Costa, Malatesta, Cipriani, Gori, Berneri, Borghi, Bompiani, Milano, 1973, pp. 167-192.

${ }^{371} \mathrm{~S}$. Fedele, Il retaggio dell'esilio. Saggi sul fuoruscitismo antifascista, Rubbettino, Sovaria Mannelli, 2010, pp. 9-10. Per uno sguardo globale sul fuoruscitismo in terra di Francia si vedano almeno S. Tombaccini, Storia dei fuorusciti italiani in Francia, Mursia, Milano, 1988 e L'Italia in esilio. L'emigrazione italiana in Francia tra le due guerre, Archivio Centrale dello Stato et al., Presidenza del Consiglio dei Ministri - Dipartimento per l'informazione e l'editoria, Roma, 1984.

${ }^{372}$ C. Berneri, La Comune di Parigi e l'idea federalista, «Culmine», II, 27 aprile 1926, riprodotto in Id., Il federalismo libertario, a cura di Patrizio Mauti, La Fiaccola, Ragusa, 1992, pp. 43-46. 
degno di nota non solo perché Berneri torna a parlare di federalismo, ma anche perché la sua interpretazione si discosta per l'ennesima volta da quella tradizionalmente proposta dagli anarchici, che da sempre considerano e celebrano la Comune di Parigi come uno degli eventi più significativi nella storia del proprio movimento.

Secondo il lodigiano, invece

Indubbiamente la rivoluzione del 1871 ebbe carattere autonomista e federalista. Ma occorre distinguere l'idea dal fatto, distinguere, cioè, quel che la Comune pensò e quel che essa fece pensare. [...]

A chi esamini attentamente i proclami, i decreti, i giornali della Comune non può apparire che evidente il contrasto tra $i$ due aspetti fondamentali di quell'avvenimento: il patriottismo suo esasperato e democratico e il socialismo rivoluzionario, nelle sue varie tendenze. Analogo contrasto si rivela nei riguardi strettamente politici, e si polarizza in due correnti: l'autoritaria, od accentratrice, e [la] libertaria, o federalista ${ }^{373}$.

Proseguendo, Berneri sostiene che

la Comune di Parigi fu autonomista e federalista, ma più nel senso che a queste parole davano Cattaneo e Ferrari che non nel senso che dava ad esso Bakunin. Ciò non toglie che essa segnasse per il movimento federalista libertario l'inizio di un florido sviluppo [...] e la possibilità di precisare il proprio sistema su quello che apparve, e in gran parte fu, il più grande modello storico di una rivoluzione antistatale e di una riorganizzazione su basi autonomiste e federaliste di carattere autodemocratico ${ }^{374}$.

Oltre al ritorno del concetto di autodemocrazia quale sistema politico di tipo federalista basato su ampie autonomie degli organi di base, atte a permettere il massimo coinvolgimento possibile dei cittadini - sul quale egli si era soffermato tra 1919 e $1921^{375}$ - la lettura berneriana è interessante poiché vede nella Comune non il modello di organizzazione che gli anarchici dovrebbero cercare di proporre, ma solo il punto di partenza per la propria riflessione politica attraverso cui giungere, infine, all'elaborazione di un'organica soluzione federalista e libertaria. Berneri sottopone insomma a critica un altro degli archetipi dell'anarchismo, un'altra delle formule che il movimento ha fatto proprie trasformandole in riferimenti astratti e riproponendole in maniera ostinatamente astorica; di nuovo, il rifiuto delle vaghezze e il richiamo ai fatti concreti.

Da rilevare inoltre come, in maniera indiretta, il lodigiano stia di nuovo spingendo gli anarchici a confrontarsi con le idee e le elaborazioni politiche di area repubblicana: sostenendo che la Comune, per quanto anti-statale, sia stata più vicina ai modelli prospettati da Cattaneo e da Ferrari, e suggerendo che essa rappresenti

${ }^{373}$ C. Berneri, La Comune di Parigi e l'idea federalista cit. I corsivi sono originali.

${ }^{374}$ Ibid.

${ }^{375}$ Vedi supra . 
comunque la base da cui gli anarchici possono partire per precisare le proprie idee federaliste, Berneri sta di fatto ribadendo un concetto già più volte espresso, ossia la necessità per il suo movimento di confrontarsi e dialogare con chi - come i repubblicani - abbia mostrato interesse a superare lo status quo attraverso elaborazioni federaliste e autonomiste.

\section{Rilanciare il movimento anarchico}

Berneri si dovrà però confrontare con un movimento anarchico italiano in esilio difficile da ricucire e riorganizzare, in una situazione di frammentazione che riflette per certi versi la crisi generale dell'antifascismo italiano, che in quegli anni ha però trovato un'importante piattaforma organizzativa nella Concentrazione Antifascista ${ }^{376}$. Berneri proseguirà negli anni dell'esilio quella che era stata la sua battaglia in Italia, in cerca di una definizione precisa e concreta di un programma libertario che fosse praticabile e sulla cui base organizzare l'azione rivoluzionaria. È sua opinione, oltreché idea diffusa nell'ambiente anarchico, che nella lotta antifascista si debba evitare di fare affidamento sulle vecchie forze politiche della democrazia liberale, ritenute responsabili principali del trionfo del fascismo. La critica maggiore è rivolta proprio alla Concentrazione antifascista, simbolo di un'opposizione legalitaria, inconsistente e figlia della sterile secessione aventiniana. La lotta contro il regime deve essere dunque una lotta rivoluzionaria, identificabile con la rivoluzione sociale in senso stretto ${ }^{377}$; Berneri ritiene pertanto necessaria un'organizzazione che non solo rilanci il movimento anarchico in esilio, ma che contribuisca anche al formarsi di un ampio fronte d'azione rivoluzionaria antifascista alternativo a quello della Concentrazione.

Il lodigiano esprime gran parte di queste idee nelle colonne dello storico quindicinale bilingue di Ginevra «Le Réveil communiste-anarchiste/Il Risveglio comunista-anarchico», e in uno di essi, intitolato L'antifascismo in Francia, si esprime così:

${ }^{376}$ La Concentrazione Antifascista, nata a Parigi nel 1927, fu un'aggregazione di diverse forze politiche ricostituitesi in esilio che tentò di proporre un'unificazione degli antifascisti fuoriusciti. Vi aderirono repubblicani, socialisti, socialriformisti, la Lega italiana dei diritti dell'uomo, la Confederazione Generale del Lavoro e, infine, anche il movimento di Carlo Rosselli «Giustizia e Libertà». Per un inquadramento generale è ancora indispensabile S. Fedele, Storia della Concentrazione Antifascista, 1927-1934, Feltrinelli, Milano, 1974.

${ }^{377}$ Sull'attività degli anarchici italiani in esilio si vedano in generale F. Giulietti, Il movimento anarchico italiano nella lotta contro il fascismo, 1927-1945, Lacaita, Manduria-Bari-Roma, 2003; G. Manfredonia, Gli anarchici italiani in Francia nella lotta antifascista, in La resistenza sconosciuta. Gli anarchici e la lotta contro il fascismo, Zero in condotta, Milano, 2005, pp. 85-113; L. Di Lembo, Guerra di classe e lotta umana. L'anarchismo in Italia dal Biennio rosso alla Guerra di Spagna (1919-1939), Edizioni Biblioteca Franco Serantini, Pisa, 2001, pp. 161-187; e G. Cerrito, L'emigrazione libertaria italiana in Francia nel ventennio fra le due guerra, in B. Bezza (a cura di), Gli italiani fuori d'Italia. Gli emigrati italiani nei movimenti operai dei paesi d'adozione, 1880-1940, Franco Angeli, Milano, 1983, soprattutto le pp. 880-911. 
Quando in ogni centro dell'emigrazione vi saranno nuclei di uomini ben preparati, nei quali al di sopra dei dissensi teorici ci sarà il desiderio di agire, si formeranno, automaticamente, delle alleanze politicamente eterogenee ma ben cementate dalla reciproca stima e dal lungo affiatamento. [...]

Opere di soccorso in favore delle vittime del fascismo, intese sindacali e politiche di carattere internazionale, cooperazione sul terreno della propaganda antifascista orale e scritta: questo può essere, e in parte lo è già, un vasto campo di collaborazione ${ }^{378}$.

E più avanti, nello stesso articolo, lancia una prima esortazione ai suoi compagni:

Bisogna metterci all'opera. Bisogna organizzare le nostre file, sì che gli impazienti, e sono i migliori, non caschino in nuovi errori [...]. L'unica forza che può servire alla profilassi delle deviazioni e alla cura ricostituente del pensiero e della volontà del nostro movimento è una salda organizzazione nostra, in seno alla quale sorgano quei nuclei di uomini d'azione che [...] potranno svolgere quell'azione sistematica e coerente insieme, che sola potrà dare il vero fronte unico rivoluzionario ${ }^{379}$.

L'azione è dunque il primo dei problemi, riorganizzarsi per Berneri significa innanzi tutto ritrovare la forza di opporsi in modo manifesto e inequivocabile al regime fascista con mezzi insurrezionali. Il legame tra fuorusciti in Francia e militanti clandestini rimasti in Italia è però labile e l'isolamento di questi ultimi particolarmente marcato; eppure non può esserci, secondo il lodigiano, soggetto politico migliore del movimento anarchico per guidare una tendenza che rompa con la strategia adottata dalla Concentrazione:

In ogni partito di sinistra s'è maturata la convinzione della limitata efficacia della critica politica e della necessità dell'azione diretta. Ma la convinzione non è interamente chiara, ché abitudini mentali le si sovrappongono e la intorbidiscono talmente che il cianciare di azione diretta è grande, sì, ma molto più grande è il battagliare cartaceo, e la critica di dettaglio e scandalistica prevale sul delineare $\mathrm{i}$ limiti e fissare gli obiettivi di una forza rivoluzionaria [...]: tutto questo ed altro dà all'antifascismo regolare ed ufficiale il marchio delle sue origini, il segno delle sue abitudini mentali e delle sue insufficienze politiche ${ }^{380}$.

Condizione necessaria, secondo Berneri, è però che una folata di attivismo ritorni all'interno del movimento:

${ }^{378}$ C. B. [C. Berneri], L'antifascismo in Francia, «Il Risveglio comunista-anarchico», XXVI, 5 marzo 1927.

${ }^{379}$ Ibid.

${ }^{380}$ C. Berneri, La nostra ora, «Il Risveglio anarchico», XXVII, 18 maggio 1929. I corsivi sono originali. 
Non basta irridere, protestare, ammonire; non basta la critica, di contro al fascismo e a quell'antifascismo equivoco e poltrone che detestiamo quanto il primo. Bisogna drizzarsi contro il fascismo, armati di una volontà, di un coraggio, di una fede che faccia volgere verso di noi tutti quelli che attendono un esempio, un grido di guerra di combattenti in guerra.

Nessun giornale clandestino diffonde in Italia la nostra parola. Basta pensare a questa lacuna perché risalti l'insufficienza della nostra azione antifascista.

Scuotiamoci dal tedio di un'attesa imbelle indegna di noi! Chiunque di noi faccia quello che può fare. Mobilitiamoci! Di fronte alla grandezza del dramma di un popolo che si adatta, si risollevi, si esalti, si coordini la nostra azione ${ }^{381}$ !

La situazione di impasse è ben avvertita da Berneri e certo influisce nel farlo tornare ad approfondire la sua ricerca di un programma minimo per la rivoluzione antifascista. Ritroviamo così il suo insistere sulla necessità che il movimento anarchico studi i problemi di tale rivoluzione, riprendendo quei motivi concretisti derivati dalla lezione di Salvemini - che erano nel tempo divenuti caratteristici del suo pensiero e della sua militanza:

Non ridicolo smaniare di tremende punizioni, non dottrinario fantasticare di dantesche apocalissi e successive utopie kropotkiniane, non presuntuosa messa in vetrina di glorie che appartengono a chi le ha meritate e a chi le ha spiritualmente preparate e non a noi tutti, ma: esame serio, cioè realistico, dei problemi della rivoluzione italiana; critica intelligente del fascismo con esame dei suoi precedenti necessari nel pseudo-liberalismo; esame dei mezzi e dei modi migliori per abbattere il fascismo; e, soprattutto, preparazione alla lotta antifascista.

[...] Vi sono dei giornali che si dicono anarchici e che sono quasi interamente dedicati a salvare il movimento. È una miseria intellettuale e morale, alla quale solo un'economia di sforzi ed un generale risveglio del movimento può impedire di esercitare un'azione disgregatrice e debilitante ${ }^{382}$.

\section{Conclude Berneri:}

Non basta continuare a pensare che con noi è la luce delle verità politiche e sociali. Occorre sentire che dovere dell'anarchico è lottare contro la tirannide. [...]

L'ora è buia. Ma è l'ora nostra. A noi la virtù e l'orgoglio di saperla afferrare. Se vinceremo la notte, potremo far nostra l'aurora. Se no, no ${ }^{383}$.

Nel suo appello finale, l'anarchico lodigiano lascia intravedere che in gioco non c'è semplicemente la sconfitta del regime: se il movimento saprà proporsi come elemento guida di un fronte rivoluzionario di azione antifascista e saprà elaborare con la dovuta serietà un suo programma, potrebbero crearsi i margini per l'attuazione e la realizzazione di una società di liberi ed eguali.

È dunque inevitabile che Berneri torni a rifletterci sopra.

\footnotetext{
${ }^{381}$ Ibid.

${ }^{382}$ Ibid. I corsivi sono originali.

${ }^{383}$ Ibid.
} 
Programma minimo anarchico e rivoluzione antifascista: la questione meridionale

Gli studi sino ad oggi condotti su Berneri non hanno considerato con sufficiente attenzione il riproporsi ampio e insistente nella sua riflessione, durante il periodo del fuoruscitismo, dei temi del federalismo e dell'autonomia. Si tratta invece di tematiche importanti, cruciali per comprendere la successiva evoluzione e gli sviluppi del suo pensiero e della sua proposta politica: nella sua battaglia problemista - influenza salveminiana da lui costantemente agitata all'interno del movimento anarchico - sono senza dubbio tra le sementi più importanti, che hanno contribuito a far germogliare come una pianta, che il lodigiano ha ritenuto possibile far crescere ancora più forte, florida e vigorosa operando alcuni innesti; questi ultimi, come abbiamo visto, provengono da movimenti, correnti e personalità politiche affini cui egli aveva nel tempo rivolto la sua attenzione. Se il suo manoscritto che invitava i compagni a preoccuparsi di elaborare un programma di azione comunalista del 1926 era rimasto nel cassetto, non altrettanto può essere detto della sua idea sulla necessità per il movimento anarchico di darsi quel programma minimo che avesse nelle autonomie comunali e nel federalismo il proprio perno politico. Berneri torna dunque a far emergere questi temi e a spronare $\mathrm{i}$ suoi compagni affinché si facciano carico di studiare tali questioni, così da poter avere le idee chiare nel momento in cui la rivoluzione antifascista prenderà finalmente le mosse.

Sul quindicinale anarchico parigino «La Lotta Umana» compare tra settembre e ottobre del 1928 un suo articolo dal titolo Nord e Sud ${ }^{384}$. Gli studiosi e i ricercatori non ne hanno fino ad oggi tenuto sufficientemente conto, ma si tratta di uno scritto estremamente significativo perché in esso ritornano non solo i suoi temi di interesse e le critiche da lui rivolte al movimento anarchico che conosciamo ormai bene, ma anche l'attenzione a modelli ed esempi politici distanti dall'anarchismo, grazie ai quali quest'ultimo potrebbe e dovrebbe arricchirsi, sviluppando finalmente delle soluzioni politiche attuabili e concrete. Berneri ritiene che i suoi compagni dell'Italia meridionale non abbiano mai raggiunto un forte e continuo sviluppo per via del «carattere generico della propaganda orale e scritta», più precisamente

I problemi agricoli dell'Italia meridionale non erano mai trattati, mancava la critica alla politica protezionistica, non venivano segnalate le sperequazioni dei tributi, delle sovvenzioni, dei lavori pubblici, ecc. tra Nord e Sud, mentre non mancavano articoli lontani ed estranei. La critica allo Stato-Autorità era meno importante, meno efficace di quella allo Stato-Amministrazione ${ }^{385}$.

Più avanti Berneri insiste:

Il «meridionalismo» $[. .$.$] fu, generalmente, ignorato dai compagni meridionali. E$ questo fu errore gravissimo. Mentre nell'Italia meridionale non mancavano

${ }^{384}$ C. Berneri, Nord e Sud, «La Lotta Umana», I, 30 settembre 1928 e II, 20 ottobre 1928.

${ }^{385}$ C. Berneri, Nord e Sud, «La Lotta Umana», I, 30 settembre 1928. 
compagni intelligenti e colti, il problema meridionale non si affacciò, nei convegni e nella stampa, che per merito di alcuni compagni del Nord, sì che l'opera di di informazione e di critica degli anarchici fu minima rispetto a quella di altri movimenti e partiti ${ }^{386}$.

Il riferimento finale non può non far venire in mente Salvemini, da sempre vivace e polemica voce meridionalista ${ }^{387}$, che difatti Berneri non esita a chiamare direttamente in causa con un esplicito riferimento:

mentre «L'Unità» di Salvemini raccoglieva accuratamente e commentava saporitamente gli sperperi economici dell'intervenzionismo statale, non uno dei nostri giornali del Mezzogiorno ne parlava, non uno dei nostri compagni meridionali pensava di farne oggetto di propaganda, a mezzo opuscoli, volantini, ecc. [...] E ai congressi della U.A.I. non ci fu un meridionale che si mostrasse al corrente dei problemi del mezzogiorno, sia nel promuovere la discussione sia nel parteciparvi.

Così $i$ compagni meridionali rimasero estranei alle critiche all'ottimismo kropotkiniano nel trattare i problemi del comunismo rurale, mentre essi avrebbero dovuto essere i primi a muoverle o a far loro $\mathrm{eco}^{388}$.

Berneri sta insomma riesumando tutti i motivi che hanno caratterizzato la sua produzione scritta prima che il fascismo lo costringesse a espatriare: critica allo Stato centralizzato quale cattivo e irresponsabile amministratore piuttosto che al principio d'autorità che esso rappresenta, rifiuto di quanto risulta inattuale e non trasferibile nella pratica del verbo dei grandi pensatori anarchici del passato, adozione di un approccio critico e confronto diretto coi problemi come metodo per elaborare soluzioni anarchiche che risultino realisticamente percorribili.

Il lodigiano non si limita tuttavia a una disamina del passato del suo movimento e rivolge piuttosto l'attenzione a cosa fare per l'avvenire, augurandosi che

ritornati in Italia, siano fondati nel Mezzogiorno dei giornali nostri che, oltre avere motivi generali, teorici e di attualità, di propaganda, attingano, con un'attenta lettura dei quotidiani, delle riviste ( $L$ La Critica Politica» sarebbe stata una miniera), dei libri sul problema agrario e sul decentramento amministrativo, elementi di propaganda contro lo Stato accentratore. Ad esempio, l'antiparlamentarismo degli anarchici meridionali s'è sempre limitato alla generica propaganda astensionista in tempo di elezioni, mentre un motivo efficace poteva essere quello espresso dal Cattaneo: «Un Parlamento centrale e un Governo unico non potranno mai occuparsi, ogni giorno, ogni ora, con affannosa sollecitudine della Sardegna, della Lombardia, della Sicilia...»

\footnotetext{
${ }^{386}$ Ibid.

${ }^{387} \mathrm{Si}$ vedano in particolare gli scritti raccolti in G. Salvemini, Opere IV, Il Mezzogiorno e la democrazia italiana, vol. II, Movimento socialista e questione meridionale, Feltrinelli, Milano, 1963.

${ }^{388}$ C. Berneri, Nord e Sud, «La Lotta Umana», I, 30 settembre 1928. I corsivi sono nostri.
} 
Criticando il sistema parlamentare accentrato, si fa il miglior antiparlamentarismo, la migliore propaganda astensionista ${ }^{389}$.

Se prima era stato Salvemini a ritornare, adesso è la tradizione federalista repubblicana a riemergere attraverso una citazione da Cattaneo e un riferimento al quindicinale di Oliviero Zuccarini, quali insostituibili esempi di fonti di studio attraverso cui rinvigorire e attualizzare il movimento e la battaglia politica degli anarchici.

Insomma, secondo Berneri, la «propaganda per la massima autonomia comunale, unita a quella contro le consorterie, [...] sarebbe feconda di educazione politica, gioverebbe all'anarchismo e avvierebbe all'Anarchia»; pertanto bisogna lottare contro l'accentramento opponendogli «un sistema tecnico-rappresentativo discentrato, autonomista e federalista insieme ${ }^{390}$. Il lodigiano si spinge fino a sbozzare un programma anarchico per il Mezzogiorno così articolato:

$1^{\circ}$ combattere ogni progetto che abbia per condizione di realizzarsi la coazione a mezzo di decreti, gendarmi, e un'armata di burocrati; $2^{\circ}$ facilitazione del trapasso della proprietà fondiaria ai coltivatori singoli o associati con l'occupazione delle terre, la formazione di consigli di contadini, l'intesa con le organizzazioni e i comuni del Centro e del Nord; $3^{\circ}$ fiducia nella forza di concorrenza dell'economia associata e individualista, e organizzazione autonoma comunale, per gli affari del comune, provinciale per quelli della provincia, regionale per quelli della regione; $4^{\circ}$ nessuna ingerenza degli organi centrali, che debbono essere soltanto organi di collegamento, di consultazione, con potere puramente esecutivo ${ }^{391}$.

Si tratta, come si può vedere, di una prima elaborazione estremamente originale di federalismo libertario organizzato attraverso organi tecnici volti a sviluppare sinergie economiche tra i Comuni e caratterizzato dalla convivenza tra soluzioni collettiviste e piccola proprietà; una soluzione che, seppure limitata in questo articolo a una proposta per la sola Italia meridionale, è assai probabile che Berneri immaginasse come estendibile a tutta la penisola. Un programma del genere avrebbe, secondo l'anarchico lodigiano, un grande vantaggio, poiché permetterebbe al movimento di porsi «in una posizione originale, tra la genericità del programma massimalista, la tecnicista e statalista soluzione comunista, e il gradualismo legalitario dei liberali e dei socialisti riformisti» ${ }^{392}$; questo significherebbe poter diventare un soggetto politico di riferimento per tutto quell'antifascismo refrattario tanto a una restaurazione dell'ordine pre-fascista quanto al trionfo delle forze comuniste o socialiste di ispirazione marxista e centralista. Sarà però necessario rompere il proprio isolamento e cominciare a porsi il problema di come

\footnotetext{
${ }^{389}$ C. Berneri, Nord e Sud, «La Lotta Umana», II, 20 ottobre 1928. I corsivi sono nostri.

${ }^{390}$ Ibid.

${ }^{391}$ Ibid.

${ }^{392}$ Ibid.
} 
condizionare la cooperazione con gli altri [movimenti e partiti] alla massima libertà politica; non solo la nostra, ma quella di altri.

Se sapremo creare intorno ai nostri nuclei un vasto alone di partigiani, potremo esercitare una notevole influenza in senso autonomista e liberista, e lasciare aperta più di una via ad ulteriori sviluppi della rivoluzione ${ }^{393}$.

Il problema prioritario, sembrerebbe suggerire il Berneri, non è tanto realizzare immediatamente l'anarchia o una società organizzata anarchicamente, bensì riuscire a essere influenti nell'emarginare tutti quei partiti e movimenti politici le cui soluzioni riporterebbero all'edificazione di uno Stato accentrato; determinare insomma uno sviluppo della rivoluzione antifascista in un senso quanto più possibile federalista e libertario. Nella conclusione dell'articolo emerge inoltre come un'aggregazione di forze politiche intorno a un programma minimo così delineato e $\mathrm{i}$ rapporti con esse costituiscano un'ulteriore questione da affrontare per gli anarchici, che certo non potranno pretendere di essere capaci di vincere da soli e che dovranno collocarsi in una sorta di fronte comune: le realizzazioni potrebbero allora non essere integralmente anarchiche, ma la nuova organizzazione lascerebbe ampi spazi di autonomia e di manovra attraverso cui il movimento potrebbe progressivamente realizzare i propri postulati.

\section{Programma minimo anarchico e rivoluzione antifascista: una battaglia autonomista}

Se il movimento anarchico in esilio è in generale favorevole a rompere il proprio isolamento e a seguire con interesse ogni movimento o corrente politica prossima a contenuti libertari, Berneri è certo l'elemento più attivo e disponibile nel ricercare contatti con gli elementi più dinamici, innovativi e pragmatici dell'antifascismo militante. La sinistra repubblicana in particolare - da tempi non sospetti, peraltro - è uno dei soggetti con cui l'anarchico lodigiano ritiene vi siano maggiori possibilità di convergenza teorica e di collaborazione pratica in senso rivoluzionario, in particolare per il loro legarsi e reinterpretare l'eredità di Mazzini, di Ferrari e di Cattaneo - figure importanti nella formazione berneriana, nonché influenti nella sua prospettiva politica federale. Non sorprende dunque trovarlo nel 1928 insieme al repubblicano Schettini (pseudonimo di Silvio Bettini) e al socialista Alberto Jacometti a dar vita a Parigi al periodico «L'Iniziativa», con la speranza di avviare un proficuo scambio attraverso cui raggiungere una vera e propria intesa in senso rivoluzionario da opporre a alla Concentrazione. Nonostante il fallimento del progetto, Berneri continuerà a guardare ai repubblicani di sinistra come a una forza politica con cui gli anarchici avrebbero dovuto dialogare e con cui sarebbe stato possibile giungere a un accordo di unità d'azione ${ }^{394}$.

Nell'agosto 1929 il lodigiano torna a occuparsi del tema dell'autonomismo con un articolo pubblicato sul mensile anarchico ticinese «Vogliamo!», intitolato Per le

\footnotetext{
${ }^{393}$ Ibid.

${ }^{394}$ Sull'esperienza de «L'Iniziativa», vedi G. Manfredonia, Gli anarchici italiani in Francia cit., pp. 103-104, e C. De Maria, Camillo Berneri cit., pp. 34-36.
} 
autonomie locali ${ }^{395}$, Berneri denuncia il soffocamento delle autonomie locali da parte del regime fascista, un'operazione che a suo dire è stata agevolata dal fatto che «tali autonomie erano ristrette e confuse, perché il governo era unitario, perché conservazione borghese e accentramento statale furono connessi, nell'indirizzo monarchico-unitario, in tutto il periodo di formazione e di rassodamento nazionale ${ }^{396}$. Nessuno studio sin qui condotto sul pensiero berneriano ha mai messo in luce come questa interpretazione del fascismo, quale esito ultimo di un percorso avviatosi con la vittoria delle forze reazionarie e con il prevalere della soluzione monarchico-unitaria del moto risorgimentale, costituisca non solo uno sviluppo di quanto già sostenuto dal maestro Salvemini nel suo saggio Le origini della reazione del $1899^{397}$, ma anche una sua sostanziale convergenza con l'interpretazione cosiddetta «rivelazionistica» che ne dettero i repubblicani nel periodo compreso tra 1927 e $1929^{398}$. Una prossimità di vedute non indifferente, che suggerisce una volta di più non solo il forte ed evidente permanere dell'influenza del professore pugliese sul pensiero politico di Berneri, ma anche la sua vicinanza agli ambienti repubblicani, con cui di fatto condivide anche analisi e riflessioni sul fascismo.

Il lodigiano tuttavia è molto più interessato a tornare sui contenuti comunalisti e federalisti che gli anarchici dovranno dare alla rivoluzione. Lo scopo finale dovrebbe essere, dal suo punto di vista, quello di rendere i Comuni «degli organi di sintesi amministrativa locale e di cooperazione, regionale e nazionale, con gli altri Comuni $\rangle^{399}$. Berneri ritorna a citare quella che era stata una sua vecchia fonte di studio e ispirazione, il de Tocqueville che «nella sua mirabile opera De la Démocratie en Amerique, ha detto ed illustrato questo assioma politico: "è nel Comune che risiede la forza dei popoli liberi". Contro la centralizzazione unitaria bisogna opporre la grande idea dell'autonomia. Alla base, i Consigli operai, contadini, impiegatizi, professionali $\rangle^{400}$. È il ritorno, ancora una volta, di quell'idea di auto-democrazia che Berneri ha iniziato a delineare sin dal 1919, ma il programma che l'anarchico lodigiano desidera portare all'attenzione dei suoi compagni si sta ora facendo ancor più chiaro e nitido per quel che riguarda il funzionamento del Comune all'interno della federazione libertaria:

Il Comune, consiglio comunale elettivo con potere esecutivo, cioè con consultazione plebiscitaria per tutto quanto riguarda interessi gravi della popolazione, con potere deliberativo, raffrenato dalla revocabilità della carica e dall'annullamento plebiscitario delle decisioni, per gli interessi minori. Il Consiglio provinciale, eletto e

\footnotetext{
${ }^{395}$ C. Berneri, Per le autonomie locali, «Vogliamo!», I, 1 agosto 1929, riprodotto in Id., Il federalismo libertario cit., pp. 51-54.

${ }^{396}$ Ibid.

${ }^{397}$ Un Travet [G. Salvemini], Le origini della reazione, «Critica Sociale», VIII, 1 luglio e 1 agosto 1899, riprodotto in G. Salvemini, Opere II, Scritti di storia moderna e contemporanea, vol. II, Scritti sul Risorgimento, Feltrinelli, Milano, 1961, pp. 13-26.

${ }^{398}$ Vedi S. Fedele, Il retaggio dell'esilio cit., pp. 37-38.

${ }^{399} \mathrm{C}$. Berneri, Per le autonomie locali cit.

${ }^{400} \mathrm{Ibid}$.
} 
controllato dai Consigli comunali. Il Consiglio regionale, eletto e controllato dai consigli provinciali. Il Consiglio nazionale, eletto e controllato dai Consigli provinciali [ma regionali, probabile refuso] ${ }^{401}$.

È un profilo ancora schematico, ma che testimonia lo sviluppo delle idee berneriane sul possibile funzionamento di un Comune che si autogoverna e si autogestisce coinvolgendo direttamente la cittadinanza sia nelle scelte che nel controllo dell'operato dei propri delegati; il tutto all'interno di un'idea federale ascendente, che dal basso procede verso l'alto, segno evidente di un'influenza federalista salveminiana che, a sua volta, deve i suoi spunti alle teorie repubblicane di Cattaneo.

Di nuovo, Berneri rileva che la cattiva e diseguale gestione accentrata del Paese fornisce «numerosi motivi di propaganda anti-statale» che possono permettere al movimento anarchico «un vasto campo di differenziazione politica da un lato e dall'altro di polarizzazione ${ }^{402}$. Inoltre, e forse è ancora più importante, gli anarchici hanno per Berneri un'enorme opportunità per operare una sintesi che superi le posizioni autonomiste e federaliste storicamente sviluppatesi tanto nel proprio movimento quanto in quello repubblicano:

I repubblicani-federalisti non potevano, nei loro sistemi, tener conto dei Consigli di fabbrica, della forza dei sindacati, dell'élite operaia. Il loro sguardo è fisso sul Comune medievale. Il federalismo Kropotkiniano, eccessivamente preoccupato della libertà individuale e allucinato dal mito del genio collettivo, si rivolge troppo a forme patriarcali di assemblee continuamente deliberanti e a forme amministrative politicamente pre-istoriche ${ }^{403}$.

Proprio per questo il movimento anarchico dovrebbe

agitare la bandiera delle autonomie. Se riuscissimo, in ogni Comune, a creare un centro di resistenza contro le forze tendenti all'accentramento statale, avremmo fatto molto. In politica, non è l'ampiezza, l'assoluta coerenza ideologica che conta, ma l'evidenza dell'utilità delle soluzioni, l'intelligenza, la costanza e l'audacia di un'aderenza alla massa qual è, di agitazioni che, volta a volta, polarizzino intorno ai nostri nuclei i bisogni e le aspirazioni delle moltitudini.

Nel campo economico l'abbiamo fatto questo passo. Rimane il campo politico ${ }^{404}$.

Programma minimo anarchico e rivoluzione antifascista: un binomio inscindibile

Alla luce di quanto sin qui riportato, ci sembra di poter dedurre e proporre una nuova interpretazione rispetto all'attività teorica e pratica messa in atto da Berneri nel corso dei primi tre anni del suo esilio: egli non sta semplicemente riproponendo al movimento anarchico i motivi e le idee che ha sostenuto a partire dal primo

\footnotetext{
${ }^{401}$ Ibid.

${ }^{402}$ Ibid.

${ }^{403} \mathrm{C}$. Berneri, Per le autonomie locali cit.

${ }^{404}$ Ibid.
} 
dopoguerra all'interno della stampa, egli non sta solamente proponendo soluzioni politiche e incitando i suoi compagni a studiare e a lavorare per concorrere a delineare quel famoso programma minimo di stampo federalista; l'anarchico lodigiano sta di fatto proponendo una saldatura tra quest'ultimo e l'azione rivoluzionaria antifascista. In altre parole, Berneri sta elaborando allo stesso tempo non solo il programma post-rivoluzionario, ma anche il programma della rivoluzione stessa. La lotta antifascista e le realizzazioni anarchiche non dovranno procedere in maniera diacronica, bensì sincronica. Il Comune, ci sembra chiaro, sarà la base dell'insurrezione contro il regime e, al tempo stesso, il luogo ove mettere subito in pratica un programma federalista e autonomista da estendere progressivamente al resto della penisola, insieme all'avanzare della rivoluzione antifascista. La nuova Italia liberata e libertaria sorgerà direttamente dai Comuni, per questo sarà cruciale che essi diventino al tempo stesso organismi di base di una nuova organizzazione politica e sociale e centri di resistenza contro il fascismo o qualunque altro tentativo di rimettere in atto un'organizzazione accentrata dello Stato.

Nell'articolo Un aborto possibile, pubblicato sul settimanale anarchico di New York «L'Adunata dei Refrattari» il 6 settembre $1930^{405}$, troviamo ulteriore conferma alla nostra interpretazione. In esso Berneri afferma:

La rivoluzione italiana sarà quello che potrà essere: banale considerazione codesta, poiché le forze operanti al lume di un'Idea hanno nelle rivoluzioni un ruolo considerevole, e tanto più quelle forze potenzieranno la propria influenza quanto più avranno chiara visione dei fini e dei mezzi.

Il fenomeno fascista deve farci persuasi che è necessario non illuderci eccessivamente sulla forza di combattività rivoluzionaria delle masse e dei partiti e movimenti di avanguardia, che rimarrà imminente il pericolo di un ricorso reazionario, ma il fenomeno fascista è là a dimostrare che soltanto colpendo a morte la plutocrazia, soltanto riformando profondamente l'ossatura ed il funzionamento dell'amministrazione, soltanto creando delle oasi fortificate di produzione comuniste, è possibile compiere una rivoluzione che garantisca realmente $e$ durevolmente libertà e giustizia ${ }^{406}$.

Ci pare che Berneri abbia fin qui chiaramente suggerito che, affinché questo "doppio programma" si avveri, sarà necessario che il movimento anarchico non sia solo, che sappia riunire intorno a sé tutte quelle forze politiche disponibili ad accettare un programma il più possibile libertario e federalista, basato sulle più ampie autonomie. Per questo potrebbe essere necessario dover trovare delle forme d'intesa con altre forze politiche, accettare di misurarsi con problemi e questioni non immediatamente risolvibili in senso anarchico, trovare insomma un medio termine tra idea e fatto; l'importante è che la rivoluzione e la nuova organizzazione siano abbastanza libertarie da lasciare al movimento anarchico spazi di autonomia e di manovra, in modo da poter eventualmente continuare a operare per portare ogni

${ }^{405}$ C. Berneri, Un aborto possibile, «L'Adunata dei Refrattari», IX, 6 settembre 1930.

${ }^{406}$ Ibid. I corsivi sono nostri. 
realizzazione non integralmente e compiutamente anarchica verso i propri fini. Gli anarchici, insomma, dovranno accettare di far parte di un più ampio movimento rivoluzionario dove confrontarsi con altre interpretazioni dei termini libertà e autonomia, dove le loro idee e le loro proposte potrebbero essere sì tollerate, ma anche non risultare del tutto vincenti. Va da sé che più il programma del movimento risulterà articolato, funzionale e capace di offrire soluzioni tecniche concrete e praticabili, maggiori saranno le probabilità che la rivoluzione antifascista si avvii su una strada di realizzazioni anarchiche; tutto dipenderà dagli anarchici stessi.

Il binomio inscindibile tra rivoluzione antifascista e programma minimo che stiamo ipotizzando sulla base della produzione berneriana sarebbe così una prima suggestiva espressione di quello che sarà l'atteggiamento assunto dal lodigiano durante la Guerra di Spagna, dove egli sosterrà a più riprese che la guerra contro le forze reazionarie e la rivoluzione sociale fossero aspetti che dovessero procedere insieme e per nessun motivo separabili tra loro.

\section{Concretismo, non opportunismo}

Nel settembre 1930 compare nel mensile «Guerra di Classe», organo parigino dell'anarcosindacalismo italiano, un articolo intitolato L'ora dell'anarcosindacalismo $^{407}$, una lettera aperta di Berneri alla redazione. Il lodigiano torna a esprimervi una delle sue convinzioni, ossia che «l'anarco-sindacalismo sia il terreno sul quale il movimento anarchico potrà entrare $[. .$.$] nel gioco delle forze sociali \mathrm{e}$ politiche della rivoluzione antifascista ${ }^{408}$. C'è però un rischio a suo modo di vedere, un rischio rappresentato dal fatto che «non abbastanza si sia elaborata, aggiornandola con le esperienze multiple e gravi del fascismo, l'ideologia anarcosindacalista», che resterebbe ancora priva di «nette ed organiche linee programmatiche e tattiche ${ }^{409}$. Berneri, lo ricorderemo, aveva indicato, nel suo manoscritto sulla necessità di un programma d'azione comunalista, proprio il terreno sindacale come un concreto campo d'azione per la rivoluzione italiana; finalmente ha l'occasione per tracciare un'altra linea importante del suo pensiero autonomista e federalista libertario, pensiero in cui i sindacati hanno un ruolo-chiave:

La maggior parte degli anarco-sindacalisti è costituita da anarchici che sono sindacalisti in quanto vedono nel sindacato un ambiente di agitazione e di propaganda più che di organizzazione classista. E ben pochi anarco-sindacalisti si sono, quindi, posti i problemi inerenti al sindacato quale cellula ricostruttiva, quale base di produzione e di amministrazione comuniste. Ancor oggi siamo al bivio, fra l'insidia del soviettismo bolscevico e l'insidia unitaria accentratrice del confederalismo socialdemocratico ${ }^{410}$.

\footnotetext{
${ }^{407}$ C. Berneri, L'ora dell'anarco-sindacalismo, «Guerra di Classe», settembre 1930.

${ }^{408}$ Ibid.

${ }^{409}$ Ibid.

${ }^{410}$ Ibid. Il corsivo è nostro.
} 
In altre parole, gli anarchici hanno l'opportunità di costituire un'alternativa nel campo delle forze socialiste e della sinistra, un'alternativa che sviluppi un'organizzazione non centralizzata e non autoritaria a partire dal basso; il sindacato è indicato da Berneri come una delle basi di questo sistema. Ancora una volta il lodigiano deve dunque tornare a insistere affinché i propri compagni si impegnino a

formulare un programma di opposizione e di costruzione, tenendo presente $\mathrm{i}$ problemi della rivoluzione italiana. La lotta per strappare alle tendenze e forze accentratrici il massimo possibile di autonomia sindacale, nelle forme elettive e deliberative deve e nei rapporti con gli organi centrali esecutivi, non può che insterilire sul terreno nettamente antiautoritario in senso individualista e individualeggiante. La lotta contro la burocrazia in generale ed il funzionamento sindacale in ispecie deve evitare esagerazioni dannose, ma deve essere instancabilmente acuta. Il problema di uno Stato sindacale va discusso ${ }^{411}$.

Di nuovo, viene ribadita con forza l'opposizione alle degenerazione burocratiche e la necessità per gli anarchici di gettarsi nella battaglia per strappare autonomie e condurre a partire da questa trincea di gestione diretta della produzione e dell'amministrazione una guerra contro il risorgere di uno Stato centralizzato, guerra che avrà bisogno però di un programma credibile e di soluzioni concrete da offrire ai problemi che inevitabilmente sorgeranno anche nelle nuove forme di organizzazione rivoluzionaria.

L'anno seguente Berneri tornerà sull'argomento pubblicando nel numero di gennaio del medesimo periodico altri due interventi sullo stesso tema. L'articolo Mali passi o fisime $?^{412}$ pone nuovamente il problema del ruolo che potrebbe assumere il sindacato in un'organizzazione di tipo federalista e libertaria:

Piaccia o non piaccia ai Vestali dell'anarchismo purissimo, il sindacato [...] dovrà, per forza di cose, diventare un organismo più completo e fondamentale, quando si assumerà il compito di gestire la produzione e gli scambi. [...] Se la crisi italiana sboccherà in una rivoluzione a direttive socialiste, i sindacati creeranno dei consigli di fabbrica, degli uffici statistici, delle centrali commerciali, dei servizi bancari, ecc. e dovranno ristabilire la pubblica amministrazione ${ }^{413}$.

Molto più che organi di rivendicazioni sociali, molto più di organizzazioni attraverso cui portare avanti il conflitto di classe. L'obiettivo degli anarcosindacalisti, prosegue Berneri, dovrebbe essere integralmente rivoluzionario, vale a dire che dovrebbe essere quello di

strappare il massimo possibile di autonomia all'opera ricostruttiva dei sindacati, di affermare il carattere tecnico degli organi e delle funzioni direttive. Il Comune dovrebbe essere la coordinante dei consigli (di fabbrica, di abitazione, di consumo

\footnotetext{
${ }^{411} \mathrm{C}$. Berneri, L'ora dell'anarco-sindacalismo cit.

${ }^{412} \mathrm{Id}$., Mali passi o fisime?, «Guerra di Classe», gennaio 1931.

${ }^{413}$ Ibid.
} 
ecc.) e la sua amministrazione risultante dell'elezione di delegati tecnici e non politici. L'apoliticità: ecco la base dell'anarco-sindacalismo. Negare la politica nell'orbita di tutta la vita amministrativa è negare lo Stato nel senso politico, combattere lo Stato come governo e come accentramento di poteri e di funzioni.

L'ordine sociale che ne risulterebbe si integrerebbe con un comunalismo federativo, che permetterebbe di uscire dal bolscevismo come dal nullismo dell'anarchismo individualista ${ }^{414}$.

Osserviamo, di nuovo, il ritornare della critica allo Stato-governo e non allo Stato come complesso di organi amministrativi, quell'originale concezione anarchica dello Stato di stampo berneriano che voleva essere un tentativo di traduzione pratica del principio per cui - nella nuova organizzazione sociale - al governo dell'uomo sull'uomo si sarebbe sostituita l'amministrazione delle cose. Berneri non ha dubbi neanche sulla formula: un sistema federale dove Comuni e sindacati operano in sinergia, dove i primi operano per garantire al proprio territorio e ai propri abitanti di usufruire di tutti i servizi e di tutti i prodotti necessari, mentre i secondi agiscono per gestire e assicurare la produzione dei beni e l'erogazione dei servizi.

Il secondo articolo Fallimento o crisi?, pubblicato nello stesso numero di «Guerra di Classe» ${ }^{415}$, sviluppa ulteriormente le suggestioni che Berneri, a partire dall'esilio, è andato sempre più marcatamente esplicitando: egli punta con decisione sulla difesa del suo concretismo rivoluzionario nel tentativo di superare il momento di crisi attraversato dal movimento:

Chiuso nell'intransigenza assoluta di fronte alla vita politica, l'anarchismo puro è fuori dal tempo e dallo spazio, ideologia categorica, religione e setta. Fuori dalla vita parlamentare, fuori da quella delle amministrazioni comunali e provinciali, non ha saputo e voluto condurre delle battaglie di dettaglio, suscitanti, volta a volta, consensi; non ha saputo agitare problemi interessanti grande parte dei cittadini. [...] Se il movimento anarchico non si decide a limitare il proprio comunismo ad una pura e semplice tendenzialità, a formulare un programma italiano, spagnolo, russo, ecc. a basi comunaliste e sindacaliste; a crearsi una tattica rispondente alla complessità e variabilità dei momenti politici e sociali; a sbarazzarsi, insomma, di tutte le sue fobie, il movimento anarchico non attirerà più la gioventù intelligente $\mathrm{e}$ colta, non saprà combattere efficacemente la statolatria comunista, non potrà per lungo tempo uscire dal marasma ${ }^{416}$.

\section{Concretismo scambiato per deviazionismo}

${ }^{414} \mathrm{C}$. Berneri, Mali passi o fisime cit. I grassetti sono originali.

${ }^{415}$ Id., Fallimento o crisi?, «Guerra di Classe», gennaio 1931, riprodotto in Id., Pietrogrado 1917 Barcellona 1937. Scritti scelti, a cura di Pier Carlo Masini e Alberto Sorti, La Fiaccola, Ragusa, 1990, pp. 128-131 (col titolo Ancora sull'anarco-sindacalismo: fallimento o crisi?), in Id., Anarchia e società aperta. Scritti editi ed inediti, a cura di Pietro Adamo, M\&B Publishing, Milano, 2001 pp. 139-141, e in Id., Scritti scelti, Zero in condotta, Milano, 2013, pp. 127-129.

${ }^{416}$ Ibid. I grassetti sono originali. 
Passano pochi mesi, quattro per la precisione, e le idee di Berneri vengono attaccate senza mezzi termini dal noto esponente anarchico Luigi Fabbri. Il suo articolo Ritorno al sindacalismo? ${ }^{417}$, pubblicato sulla rivista «Studi Sociali» da lui fondata e diretta a Montevideo, non chiama in causa il lodigiano in modo diretto, ma appare chiaro che sia rivolto a lui e alle idee che ha espresso su «Guerra di Classe». Sostanzialmente convinto del fatto che il sindacalismo, sia pure di matrice anarchica, sia stato superato come metodo e come strumento di lotta e rivoluzione sociale, Fabbri lamenta non solo il fatto che vengano lanciate «molte accuse, o piuttosto frecciate contro l'anarchismo in generale, con parole spregiative che han solo per effetto di irritare inutilmente una quantità di buoni compagni», ma soprattutto che non ci si avveda che

il rimedio è stato più volte indicato, e va in ogni modo cercato e attuato nell'anarchismo stesso, su direttive anarchiche, nettamente antiautoritarie; e non in amalgama producenti confusioni e contrasti sempre nuovi, con movimenti anche legittimi ed utili, come quello sindacale, che hanno però carattere e natura loro propri e del tutto distinti e non confondibili con l'anarchismo ${ }^{418}$.

Berneri viene insomma velatamente accusato di deviazionismo, o comunque di essersi avviato su una strada sbagliata, per via del suo insistere sulle potenzialità rivoluzionarie del sindacato; ma più avanti Fabbri è ancora più esplicito:

Vi sono alcuni [...] i quali hanno accennato vagamente a non so che "stato sindacale" da proporsi come scopo della lotta anarchica, o anarco-sindacalista che sia. [...] Ma habent sua fata anche le parole; e ormai, specie nel linguaggio anarchico, la parola "stato" ha un significato troppo...governativo per prescinderne senza dar luogo a confusione. Tanto vero che ci sono di quelli che han molta tendenza a interpretare lo stato sindacale in una forma embrionale di governo, per costringere a forza i riluttanti a fare quel che vuole il sindacato. E allora sarebbe...un altro paio di maniche; noi propenderemmo in tal caso per la rivolta contro il sindacato!

Non potrebb'essere diversamente, infatti, per degli anarchici, se si prendesse l'espressione "Stato sindacale" in senso politico e positivo di organo statale o governativo [...]. In tal caso non sarebbe neppure una cosa nuova. Già nel 1920 si parlava di "tutto il potere ai sovieti", e poi di "tutto il potere ai sindacati" [...]. Ma, esaminate bene tutte queste formule, vi si scorgevan sempre tante mascherature di quella famigerata "conquista dei pubblici poteri", che gli anarchici respinsero come insidiosa, bugiarda, e traditrice del proletariato e della libertà, fin dal $1872^{419}$.

Non sembrano esserci dubbi, a questo punto, circa il destinatario di tale articolo. Per mettere un punto sulla questione, Fabbri afferma che i compagni sostenitori dell'idea dello Stato sindacale «potrebbero diventare sindacalisti quanto vogliono,

\footnotetext{
${ }^{417}$ L. Fabbri, Ritorno al sindacalismo?, «Studi Sociali», II, 12 giugno 1931.

${ }^{418}$ Ibid.

${ }^{419}$ Ibid. Il grassetto è originale.
} 
ma cesserebbero indubbiamente di essere anarchici» ${ }^{420}$. Un colpo netto che certo non può lasciare indifferente Berneri, che elaborerà dunque una risposta che però deciderà infine di non proporre per la pubblicazione e che, dopo essere rimasta inedita per anni, ha visto la luce per la prima volta solamente nel $2001^{421}$. Berneri vi difendeva la tesi già espressa, difendendosi dalle accuse di confusionismo e di equivocità:

\begin{abstract}
Noi non siamo sindacalisti puri e non siamo anarchici che non vedono tutta l'importanza del sindacato non solo come campo d'agitazione rivoluzionaria e di propaganda libertaria ma anche come principale cellula ricostruttiva, nella rivoluzione comunista, di un nuovo ordine autonomista-federalista che salvi dall'accentramento politico [...].

Nello Stato occorre distinguere il governo e tutto quel complesso di organi e di funzioni di coordinazione amministrativa e tecnica che non può non essere assorbito che dai Comuni, dai Consigli operai, dai Sindacati ${ }^{422}$.
\end{abstract}

Berneri sostiene nuovamente la distinzione tra governo e amministrazione, riportando l'attenzione sulla necessità di un programma federalista libertario, capace di emergere come alternativa percorribile e capace di fornire indirizzi e risposte alla auspicata rivoluzione antifascista e all'Italia futura:

Tra il federalismo repubblicano e il soviettismo bolscevico deve incunearsi l'anarcosindacalismo, che non è che l'anarchismo epurato del mito della rivoluzione dominata e risolta dall'“iniziativa popolare", e dei residui dell'autonomismo individualistico. L'anarco-sindacalismo deve creare un programma di ricostruzione sociale, che, ispirandosi al federalismo di Proudhon e Pisacane da un lato e dall'altro alle recenti esperienze rivoluzionarie e agli aspetti attuali del problema sociale in Italia, permetta agli anarchici di strappare il massimo possibile di autonomia per i Comuni e per le organizzazioni operaie, combattendo qualsiasi dittatura di partito e il formarsi e il consolidarsi di un nuovo Stato accentratore e politico, cioè di un nuovo governo ${ }^{423}$.

Ancora una volta, insomma, Berneri fa appello alla concretezza e alla fine degli "assoluti ideologici". La proposta da lui messa in campo ribadisce quelle posizioni valutate da Fabbri come deviazioniste, ma che invece sembrerebbero spiegabili in tutt'altra maniera: essa non costituirebbe, per il lodigiano, che uno dei primi passi concreti da muovere lungo un cammino che condurrebbe verso l'arginamento delle soluzioni di accentramento statale e che potrebbe progressivamente svilupparsi fino

\footnotetext{
${ }^{420}$ Ibid.

${ }^{421}$ C. Berneri, Risposta a Luigi Fabbri, in Idem, Anarchia e società aperta cit., pp. 190-191. La risposta di Berneri al Fabbri è stata pubblicata per la prima volta nel volume antologico di Pietro Adamo nell'unica versione ad oggi disponibile: una trascrizione della moglie di Camillo Berneri, Giovanna Caleffi.

${ }^{422}$ Ivi, pp. 190-191. Il corsivo è originale.

${ }^{423}$ Camillo Berneri, Risposta a Luigi Fabbri cit., p. 191.
} 
alla realizzazione integrale delle istanze anarchiche. Ciò conduce necessariamente ad accettare una tattica rivolta quanto meno verso il realizzarsi di un ordine federalista e libertario piuttosto che verso la pretesa di poter realizzare integralmente e immediatamente l'anarchia; in tale tensione potrebbe essere necessario accettare passaggi progressivi, nonché la collaborazione con altre forze politiche intorno a un "programma minimo".

Il federalismo è ora più che mai - ci sembra di poterlo affermare con certezza la chiave di volta del pensiero politico berneriano, il paradigma dove può essere possibile trovare un terreno pratico e operativo di realizzazioni libertarie, ove poter far convergere e coniugare eredità repubblicana risorgimentale, idee anarchiche e nuove esperienze rivoluzionarie dove la rappresentanza viene controllata "dal basso". A tal proposito è emblematica la ben nota lettera del 1929 con cui Berneri descriveva il suo anarchismo sui generis e il suo rapporto col movimento a Libero Battistelli, futuro giellista:

Ho abbandonato il movimento socialista perché continuamente mi sentivo dare dell'anarchico; entrato nel movimento anarchico mi sono fatto la fama di repubblicano federalista. Quello che è certo è che sono un anarchico sui generis, tollerato dai compagni per la mia attività, ma capito e seguito da pochissimi. I dissensi vertono su questi punti: la generalità degli anarchici è atea e io sono agnostico, è comunista e io sono liberalista (cioè sono per la libera concorrenza tra lavoro e commercio cooperativi e lavoro e commercio individuali); è antiautoritaria in modo individualista ed io sono semplicemente autonomista federalista (Cattaneo completato da Salvemini e dal sovietismo) ${ }^{424}$.

Lo stesso Salvemini in questo periodo lo sta stimolando a ricercare concretezza nella sua azione, come appare significativamente in un suggestivo e brillante passaggio di una lettera del 1932:

Si è sempre i moderati o gli estremisti di qualcuno! Tu, per esempio, sei un estremista per me e sei un moderato per molti tuoi compagni. Anche in questo l'importante è di saper scegliere il luogo dove applicare la leva: un estremista che non cava un ragno da un buco serve quanto un moderato che non si arrischia neanche a cercare il ragno ${ }^{425}$.

\section{In cerca di contatti e convergenze: $i$ repubblicani}

Berneri certamente ha ben presente che il punto cruciale sia tradurre in pratica le sue tensioni concretiste creando o trovando, appunto, «il luogo dove applicare la leva». La sua è a ben guardare una preoccupazione tra le più vive, se non la più viva, all'interno del fuoruscitismo anarchico che nel corso degli anni '30, tra le repressioni

${ }^{424}$ Lettera di Camillo Berneri a Libero Battistelli (s.l., s.d., ma probabilmente 1929), in C. Berneri, Epistolario inedito, vol. I, a cura di Aurelio Chessa e Pier Carlo Masini, Edizioni Archivio Famiglia Berneri, Pistoia, 1980, pp. 18-19.

${ }^{425}$ Lettera di Gaetano Salvemini a Camillo Berneri (Parigi, 14 febbraio 1932), in C. Berneri, Epistolario inedito, vol. I cit., p. 136. 
subite dalle forze dell'ordine francesi e il progressivo peggioramento del contesto politico internazionale, inizia a interrogarsi sul ruolo da svolgere entro la rivoluzione nazionale e sul programma da adottare per poter giocare un ruolo importante in quest'ultima. Soprattutto il problema dell'unità d'azione torna a diventare attuale e a essere particolarmente sentito poiché, rifiutando come si è detto l'alleanza con le forze del passato, si inizia a percepire in modo chiaro l'autoisolamento cui il movimento si sta condannando. Ciò nondimeno nessun elemento si dichiara favorevole ad alcuna ipotesi di fronte unico, assumendo al massimo un atteggiamento di apertura e di possibilità di dialogo con le forze antifasciste, capace di rompere tanto con la sterilità della Concentrazione, quanto con i partiti di impianto marxista.

Berneri, lo ricorderemo, è da diverso tempo che si interessa e che appare disponibile a un dialogo con la sinistra repubblicana e a riprova di ciò si può evidenziare come, a partire dal 1932, egli torni a premere affinché il movimento anarchico si dimostri aperto e possibilista nei confronti di almeno una parte degli esponenti e dei militanti del PRI. Nessuno studio ha fino ad oggi evidenziato e messo in luce in modo adeguato il fatto che l'anarchico lodigiano in questi anni sia tornato a indicare nei repubblicani di sinistra un soggetto politico con cui potesse essere possibile trovare elementi di prossimità in un momento tutt'altro che casuale: già a partire dal 1929 nel Partito Repubblicano era emersa una corrente di ispirazione rivoluzionaria guidata da Fernando Schiavetti, assai critico verso la Concentrazione antifascista e convinto di dover dare al suo partito un carattere più marcatamente sociale e classista - tale corrente si era infine affermata durante il Congresso del PRI (19-20 marzo 1932) tenutosi a Parigi ${ }^{426}$. Nel giugno di quello stesso 1932 un appunto della Polizia Politica riferisce della fondazione di un «Circolo Italiano di Coltura» organizzato, tra gli altri, da Berneri e dai repubblicani Rossetti e Schettini; dalla lettura dell'appunto apprendiamo che il Circolo

svolge attualmente un'intensa attività per raggruppare in senso alla C.I.C. tutti i gruppi e le correnti politiche antifasciste della piazza.

[...] Detto circolo, secondo le intenzioni degli organizzatori, dovrebbe rendere possibile coi contatti più frequenti tra gli esponenti dei vari gruppi antifascisti [...] un'intesa per una comune azione contro il Regime ${ }^{427}$.

Berneri deve insomma aver seguito con interesse l'evolversi del dibattito all'interno delle file repubblicane e ritiene di poter trovare in esse una possibile sponda per l'unità d'azione rivoluzionaria che sta ricercando; questa ipotesi appare inoltre suffragata da alcuni suoi articoli che escono nel corso dello stesso anno. Il

${ }^{426}$ Vedi S. Fedele, I repubblicani in esilio nella lotta contro il fascismo (1926-1940), Le Monnier, Firenze, 1989, pp. 47-64, ma anche M. Tesoro, Un leader dissidente, in E. Signori, M. Tesoro, Il verde e il rosso: Fernando Schiavetti e gli antifascisti nell'esilio fra repubblicanesimo e socialismo, Le Monnier, Firenze, 1987, soprattutto le pp. 43-56.

${ }^{427}$ Appunto della Div. Pol. Polit., 18 giugno 1932 per la Dir. Gen. PS, AA. GG. RR., Sez. Prima, in ACS, Min. In., CPC, Berneri Camillo, b. 537, fsc. III. 
primo che ci appare significativo è Del diritto alla critica, pubblicato su «L'Adunata dei Refrattari» il 2 luglio 1932 ${ }^{428}$ : esso è interessante poiché, oltre a manifestare chiaramente la simpatia berneriana per l'affermarsi della corrente guidata da Schiavetti, offre una vera e propria retrospettiva sul suo atteggiamento intellettuale e sul rapporto da lui tenuto nei confronti non solo dei repubblicani italiani, ma anche di altre importanti esperienze nel campo democratico.

Ecco come si esprime Berneri:

Tutta la mia attività intellettuale in seno al movimento nostro, comunque sia giudicabile dal punto di vista ortodosso, è stata sempre ed è tutt'ora improntata ad uno sforzo di impostazione realistica dei problemi, ad un problemismo per nulla ideologico, che ha diretto le mie simpatie verso La Critica Politica di Zuccarini, L'Unità di Salvemini e La Rivoluzione Liberale di Gobetti. E aggiungo L'Ordine Nuovo, rivista ${ }^{429}$.

A questi espliciti riferimenti alle riviste di studio e di rinnovamento culturale e politico che dimostrano, ancora una volta, quanto il dibattito italiano a lui coevo abbia influenzato la sua maturazione intellettuale e caratterizzato la sua militanza, fa seguito l'esame dei suoi rapporti coi repubblicani:

Le mie simpatie per i repubblicani revisionisti in senso socialista e autonomista, risalgono al 1919 e le ho più volte manifestate, giungendo a polemizzare con Malatesta [...]. In sede politica, il federalismo repubblicano di Cattaneo e del Ferrari mi pareva, fin dal 1918, passibile d'integrarsi col comunalismo libertario propugnato dalla Ia Internazionale e con il soviettismo, quale esperienza genuina, cioè prima che diventasse strumento della dittatura bolscevica.

La crisi del partito repubblicano, risultante dall'affermarsi della corrente socialistaautonomista, viene ad accentuare l'avvicinamento di parte dei repubblicani a noi [...] ed è naturale che io simpatizzi con chi sta elaborando un programma repubblicano che conterrà più, di socialismo e di autonomismo, di quello che contengano il vecchio programma tradizionale e il programma della Concentrazione ${ }^{430}$.

La svolta operatasi all'interno del PRI potrebbe rappresentare l'occasione per avviare quell'organizzazione auspicata da Berneri di un polo di forze politiche che dovrebbero cercare di incanalare la rivoluzione antifascista e la futura Italia liberata verso un'organizzazione di tipo federalista e libertaria.

Un altro suo articolo, pubblicato su «Guerra di Classe» nell'agosto 1932 col titolo Il problema delle autonomie locali ${ }^{431}$, conferma che egli nutrisse queste aspettative. L'anarchico lodigiano ritorna su alcuni argomenti già affrontati, come il soffocamento delle residue autonomie comunali e provinciali operato dal fascismo e

${ }^{428}$ C. Berneri, Del diritto alla critica, «L'Adunata dei Refrattari», XI, 2 luglio 1932.

${ }^{429}$ Ibid. I corsivi sono nostri.

${ }^{430} \mathrm{C}$. Berneri, Del diritto alla critica

${ }^{431}$ Id., Il problema delle autonomie locali, «Guerra di Classe», agosto 1932, riprodotto in Id., Il federalismo libertario, a cura di Patrizio Mauti, La Fiaccola, Ragusa, 1992, pp. 55-61. 
l'idea che il regime rappresenti l'esito finale del trionfo e dello sviluppo delle forze accentratrici e reazionarie all'indomani dell'unificazione italiana ${ }^{432}$; ma la novità è rappresentata dall'indice che il Berneri punta nuovamente in direzione del rinnovato PRI, indicando così ai suoi compagni un possibile interlocutore nella prospettiva di un'unione di forze disponibili a operare verso comuni finalità antifasciste e antiaccentratrici. Berneri auspica che i repubblicani, una volta accantonati e abbandonati i «misticismi mazziniani», possano elaborare un serio programma autonomista e federalista che tenga conto delle nuove esperienze politiche e sociali:

Il Soviettismo sindacalista, il Comunalismo mazziniano, il regionalismo del Cattaneo possono confluire e sboccare in un sistema che aggiorni la posizione dei Repubblicani, non solo sul terreno politico ma anche, e soprattutto, su quello sociale. Proudhon e De Tocqueville, Pisacane e Cattaneo, lo studio del Soviettismo russo; ecco un ampio campo di studio e di discussione, cioè di preparazione culturale. I Repubblicani emigrati, fino ad oggi, non hanno ripresa e continuata questa elaborazione federalista ${ }^{433}$.

Di fatto Berneri sta spingendo affinché la svolta politica e sociale dei repubblicani sia accompagnata da un ritorno allo studio della propria tradizione federalista e da un confronto con altre elaborazioni similari. In altre parole, sta invitando i repubblicani a porsi su un iter similare (se non identico) a quello che egli sta tracciando all'interno del suo movimento.

Nell'ottobre dello stesso anno l'anarchico lodigiano torna a insistere sulla svolta operatasi all'interno del PRI con l'articolo I repubblicani di destra, gli altri e noi pubblicato sul primo numero di «Umanità Nova», rinata in quel 1932 per sua iniziativa come pubblicazione quindicinale e stampata a Puteaux ${ }^{434}$. Berneri vi sostiene che il Partito Repubblicano sia guidato da elementi «giovani, sinceramente rivoluzionari ed aperti alle idee più avanzate ed attenti alle grandi trasformazioni sociali in corso ed imminenti», e più avanti precisa:

L'errore concentrazionista perpetuava l'equivoco della confusione del P.R. con il costituzionalismo liberale e social-riformista, [...] e lo imbottigliò per anni ed anni nel concentrazionismo fumogeno, come capirono Schiavetti, Volterra, Chiodini ed altri che sono, oggi, all'estrema sinistra del repubblicanesimo. La sinistra repubblicana è alla nostra destra, ma noi seguiamo con interesse e simpatia questa maturazione, memori dei legami che hanno sempre avuto gli operai repubblicani alla causa dell'emancipazione popolare. ${ }^{435}$

${ }^{432}$ Vedi supra, pp. 202-203

${ }^{433} \mathrm{C}$. Berneri, Il problema delle autonomie locali cit., p. 59.

${ }^{434}$ [C. Berneri], I repubblicani di destra, gli altri e noi, «Umanità Nova», I, 20 ottobre 1932. Sulla ripresa delle pubblicazioni della nota rivista anarchica da parte dei militanti fuorusciti in Francia si veda F. Schirone, Umanità Nova in esilio: Parigi 1932-1933, in Id. (a cura di), Cronache anarchiche. Il giornale Umanità Nova nell'Italia del Novecento (1920-1945), Zero in condotta, Milano, 2010, pp. 225-246.

${ }^{435}$ Ibid. 
Il momento è inoltre propizio per un altro motivo, poiché

Il dissidio ideologico tra i repubblicani e noi è molto attenuato, essendo ormai lontana l'epoca del duello Mazzini-Bakounine. Da un lato i repubblicani stanno liberandosi della teologia mazziniana, dall'altro noi ci siamo liberati del marxismo ${ }^{436}$.

È probabile che ciò per Berneri non costituisse una ragione sufficiente a favorire un incontro su un comune terreno di lotta, tuttavia riteniamo che si sentisse quanto meno autorizzato a sperare in uno sviluppo di questa svolta politica in direzione libertaria. Speranza che tuttavia sarà destinata a dissolversi in breve tempo: in difficoltà nel trovare una propria collocazione nella sinistra antifascista e incapace di compattare il partito sulla nuova linea politica, la corrente di Schiavetti verrà sconfitta durante il Congresso del PRI svoltosi a Parigi (22-23 aprile 1933) ${ }^{437}$.

\section{In cerca di contatti e convergenze: "Giustizia e Libertà»}

La comparsa del movimento "Giustizia e Libertà» ${ }^{438}$ - fondato a Parigi nel luglio 1929 da Carlo Rosselli, Emilio Lussu e Fausto Nitti - all'interno dell'area antifascista rappresenterà un altro fattore innovativo e di un certo interesse per Berneri e, più in generale, per il movimento anarchico. L'assenza nel movimento politico giellista di legami coi vecchi partiti, il suo essere formato da elementi giovani e la sua disponibilità all'impegno diretto con azioni dimostrative e spettacolari costituiscono non pochi punti di contatto con quelle che abbiamo visto essere le istanze del fuoruscitismo anarchico, in rottura con un antifascismo sterile e temporeggiatore. Inizialmente è soprattutto lo spiccato fermento eversivo e di rottura che anima il giellismo a risvegliare l'attenzione e l'interesse di parte degli anarchici, mettendo in secondo piano la discussione del suo programma e della sua ideologia. Chi all'interno del movimento non si lascia tentare - e tra questi vi è anche Berneri svilupperà invece gran parte della sua critica proprio su questi punti.

L'anarchico lodigiano giudicherà «Giustizia e Libertà» come una realtà sostanzialmente moderata e borghese fin dalla sua apparizione e non cambierà le sue idee per tutta la fase in cui il movimento giellista resterà legato alla Concentrazione antifascista. Emblematico in questo senso uno dei primi articoli berneriani sul tema, intitolato Il movimento "Giustizia e Libertà" e apparso su «L'Adunata dei

\footnotetext{
${ }^{436}[$ C. Berneri], I repubblicani di destra, gli altri e noi cit.

${ }^{437}$ Vedi S. Fedele, I repubblicani in esilio nella lotta contro il fascismo cit., pp. 64-75, ma anche M. Tesoro, Un leader dissidente cit., pp. 56-66.

${ }^{438}$ Per un profilo del movimento giellista si vedano i recenti studi di M. Bresciani, Quale antifascismo? Storia di Giustizia e Libertà, Carocci, Roma, 2017, e di M. Giovana, Giustizia e Libertà in Italia: storia di una cospirazione antifascista, 1929-1937, Bollati Boringhieri, Torino, 2005. Cfr. inoltre l'ancora indispensabile Giustizia e Libertà nella lotta antifascista e nella storia d'Italia. Attualità dei fratelli Rosselli a quaranta anni dal loro sacrificio, La Nuova Italia, Firenze, 1978.
} 
Refrattari» il primo novembre $1930^{439}$ : in esso Berneri si concentra proprio sul carattere troppo moderato e indeterminato del nuovo movimento, specialmente per quel che riguarda il federalismo e l'autonomismo, il campo in cui come abbiamo visto è maggiormente interessato a cercare convergenze e intese d'azione.

Egli sostiene che le esperienze rivoluzionarie

di Russia, di Germania e di Ungheria hanno, sia pure in forme embrionali e caduche, creato una democrazia nuova, neppure intravista, al di fuori dei federalisti anarchici od anarcheggianti, né dal liberalismo classico né dal federalismo repubblicano. Questa democrazia, inglobante il cittadino ed il produttore nel quadro di un ordine nuovo di libertà e di giustizia, non pare essere esistita [...] per i democratici della Concentrazione. E un generico accenno alle autonomie degli enti locali o regionali ha tutta l'aria di essere un contentino per i liberali, per i repubblicani e per i popolari, più che il riflesso di una reale tendenza ad una soluzione autonomista della vita politica del paese ${ }^{440}$.

La questione è di principio: nel momento stesso in cui GL ha aderito alla Concentrazione ha fatto segno di non essere propensa a lavorare nel senso di un programma politico di profonda riforma politica e sociale, caratterizzato da un federalismo basato sulle autonomie di una pluralità di formazioni democratiche che permettano una più ampia partecipazione e forme di autogoverno. Ciò è sufficiente per far concludere a Berneri che «la Concentrazione e l'associazione "Giustizia e Libertà" sono movimenti MODERATI» ${ }^{441}$.

Ancora due anni dopo l'anarchico lodigiano si mostrerà delle stesse identiche idee e in un nuovo articolo su «L'Adunata dei Refrattari» parlerà, a proposito del movimento giellista e della Concentrazione, di «tattica fumogena» ${ }^{442}$. Berneri teme soprattutto che i suoi compagni si lascino tentare dalle apparenze azioniste e rivoluzionarie di GL, motivo per cui torna a insistere affinché anche il movimento anarchico ritrovi unità e compattezza, tornando ad agire sullo stesso terreno su cui altri movimenti e partiti si stanno dimostrando più attivi:

Noi dobbiamo intensificare la nostra propaganda clandestina, dobbiamo organizzare dei gruppi di preparazione rivoluzionaria a tipo regionale, dobbiamo ristabilire il maggior numero di contatti con i nostri compagni rimasti in Italia ${ }^{443}$.

Necessità che peraltro il lodigiano non si è mai stancato di far presenti, ma ora si sta facendo sempre più concreto il rischio che alcuni anarchici si lascino attrarre «dai

\footnotetext{
${ }^{439} \mathrm{C}$. Berneri, Il movimento "Giustizia e Libertà", «L'Adunata dei Refrattari», IX, 1 novembre 1930.

${ }^{440} \mathrm{Ibid}$. I corsivi sono nostri.

${ }^{441}$ Ibid. Il maiuscolo è originale.

${ }^{442}$ C. B. [C. Berneri], La tattica fumogena, «L'Adunata dei Refrattari», XI, 10 gennaio 1932. ${ }^{443}$ Ibid.
} 
movimenti sedicenti rivoluzionari, che preparano i quadri ed i mezzi repressivi di una repubblica conservatrice» ${ }^{444}$.

Nel giugno del 1932 affronterà - sempre su «L'Adunata dei Refrattari» - la questione in maniera diretta, poiché anche i giellisti stanno proponendo una tattica rivoluzionaria basata su comitati locali: nell'articolo Gli anarchici e $i$ comitati locali rivoluzionari ${ }^{445}$, egli risponde su questo tema ad Alberto Meschi che in un articolo su «Il Martello» ${ }^{446}$, settimanale anarchico di New York, aveva espresso la necessità di discutere la possibilità di un'intesa rivoluzionaria tra anarchici e GL entro tali comitati locali. Berneri si oppone sostenendo non solo che GL stia accogliendo tra le sue file elementi anarchici per «dimostrare che essa è rivoluzionaria», ma anche che il suo programma sia equivoco «là dove questo parla del governo "sorto dalla rivoluzione" che insieme ai "comitati locali" porrà le basi del "nuovo governo"»; tali comitati «tenderebbero naturalmente alla dittatura politica», divenendo la base non di una nuova organizzazione politica e sociale, ma di un governo provvisorio ${ }^{447}$.

Berneri ha così modo di rilanciare un'altra volta la sua idea di unità inscindibile tra programma minimo anarchico e rivoluzione antifascista:

I comitati locali rivoluzionari ai quali potremo partecipare saranno quelli che sorgeranno al di fuori e contro tutti i partiti politici, che si propongono tutti di arrivare al governo. Quelli, cioè, che sorgeranno dalle masse operaie e contadine per la necessità di assicurare il ritmo della produzione e degli scambi e di gettare le basi di una organizzazione amministrativa comunale e corporativa. Dobbiamo proporci di sostenere questo principio fondamentale: le rappresentanze debbono essere tecniche e non politiche, debbono formarsi in base alle capacità direttive e all'onestà, debbono essere passibili di controllo e di annullamento da parte di tutti gli interessati ${ }^{448}$.

Prosegue più avanti Berneri:

Io sono per un programma minimo, ma quando vedo il "revisionismo" giungere all'accettazione del governo provvisorio e della dittatura politica, alla sfiducia dell'azione specifica dell'anarchismo nella rivoluzione, al collaborazionismo accomodante, mi domando se certi “compagni” sono anarchici o sono degli elementi

${ }^{444}$ C. B. [C. Berneri], La tattica fumogena cit.

${ }^{445} \mathrm{C}$. Berneri, Gli anarchici e $i$ comitati locali rivoluzionari, «L'Adunata dei Refrattari», XI, 18 giugno 1932, segue nota della redazione.

${ }^{446}$ A. Meschi, Gli anarchici e "Giustizia e Libertà", «Il Martello», XVII, 2 aprile 1932. Alberto Meschi (1879-1958) è stato un militante anarchico italiano nativo di Borgo San Donnino, odierna Fidenza. Noto soprattutto per la sua attività da segretario della Camera del Lavoro di Carrara, di cui favorisce il rilancio a partire dal 1911, nel 1922 è costretto a espatriare in Francia. Da fuoruscito si profonde intensamente nell'attività antifascista. Vedi «Meschi, Alberto», in M. Antonioli et al. (diretto da), Dizionario biografico degli anarchici italiani, vol. II, Edizioni Biblioteca Franco Serantini, Pisa, 2004, pp. 170-172.

${ }^{447} \mathrm{C}$. Berneri, Gli anarchici e i comitati locali rivoluzionari cit.

${ }^{448}$ Ibid. 
che non hanno una chiara visione dei nostri fini, una sufficiente fiducia nei nostri mezzi e, più ancora, un temperamento libertario ${ }^{449}$.

Appare chiaro che, nonostante la sua posizione sui generis, l'anarchico lodigiano non sia affatto disponibile a spingere i sacrifici delle idee anarchiche oltre un certo limite e come sia intenzionato a mantenere il suo programma minimo entro confini quanto più possibile coerenti, precisi e nitidi; condizione necessaria, ribadita ancora una volta, è che nel movimento si avvii una «serena e obiettiva discussione sui problemi della rivoluzione italiana» ${ }^{450}$. Non altrettanto chiaro appare però alla redazione de «L'Adunata dei Refrattari», che, in una nota redazionale apposta all'articolo, esprime alcune perplessità circa quanto scritto da Berneri:

Il compagno Berneri è per un "programma minimo". Noi non sappiamo che cosa si possa intendere per programma minimo dell'anarchia. Ma se il programma minimo del Berneri è un programma anarchico, cioè senza transazioni antiautoritario, antistatale, noi non riusciamo a comprendere in che cosa possa differirsi dal "programma massimo" dell'anarchia [...]. Se invece il "programma minimo" del compagno Berneri fosse un altro tentativo di compromesso tra l'anarchia e lo Stato, tra la libertà e l'autorità, un anarchismo a scartamento ridotto innestato alla radice di un governo ridotto ai minimi termini, allora egli cadrebbe nelle stesse deviazioni in cui si diletta il Meschi $[\ldots]^{451}$.

Berneri non chiarificherà la sua posizione rispondendo alla redazione de «L'Adunata dei Refrattari», ma i nodi verranno presto al pettine.

\section{Per un'Italia federalista e libertaria: la Costituente}

Giunti a questo punto, riteniamo di poter affermare che la domanda che nessuno studioso si è adeguatamente posto e a cui, conseguentemente, nessuna ricerca di storia del pensiero politico ha saputo finora dare risposta è come mai un anarchico dovrebbe sentire la necessità di redigere una Costituzione. Berneri si sarebbe potuto limitare a definire i contorni del proprio programma minimo, proporlo e discuterlo coi propri compagni o con gli esponenti e i militanti di movimenti e partiti con cui si sarebbe ritenuto possibile trovare un'intesa; ciò avrebbe dato al piano di rivoluzione antifascista e di riorganizzazione su basi federaliste e libertarie dell'Italia un carattere più flessibile e dinamico, di certo meno prescrittivo e normativo di una carta costituzionale. Insomma, un anarchico - e Berneri lo è, ci sentiamo di poterlo sostenere senza ombra di dubbio - dovrebbe per definizione respingere e detestare l'idea di una Costituente, di un documento che formalizzi nero su bianco le norme

\footnotetext{
${ }^{449}$ Ibid.

${ }^{450} \mathrm{Ibid}$.

${ }^{451}$ Nota della Redazione a C. Berneri, Gli anarchici e i comitati rivoluzionari cit.
} 
che stanno alla base di una comunità politica e sociale e che ne regolano il funzionamento.

A suo tempo l'autorevole anarchico Malatesta, con l'articolo A proposito di Costituente apparso su «Pensiero e Volontà» il 15 ottobre 1924, aveva pronunciato parole esplicite in questo senso, opponendosi a tale idea in quanto ennesima occasione in cui si deve accettare una «maggioranza che s'impone alla minoranza, che vota la costituzione a maggioranza, e l'impone con la forza ai dissidenti, che potrebbero poi essere la maggioranza reale ${ }^{452}$. Per tale ragione gli anarchici dovrebbero opporre alla Costituente o ad altri corpi legislativi

dei Congressi, delle Convenzioni, locali, regionali, nazionali, le quali saranno aperte a tutti per informare, consigliare, prendere delle iniziative - senza la pretesa di far la legge e d'imporre agli altri con la forza le proprie deliberazioni ${ }^{453}$.

Berneri avrebbe potuto semplicemente aderire a questo indirizzo - che è sostanzialmente quanto gli abbiamo sentito dire e affermare a più riprese fin qui - e proseguire su questa linea la sua battaglia di rinnovamento; il perché a un certo punto abbia atteso alla stesura di un progetto di carta costituzionale è rimasto fuori dalle indagini, risultando pertanto ancora inspiegabile e inspiegato.

Per comprendere lo sviluppo della riflessione berneriana, riteniamo però che la questione vada spostata e che sia necessario anzitutto capire cosa Berneri intendesse per Costituzione mentre pensava e redigeva la sua costituente libertaria: alla luce di quanto emerso nel corso di questa ricerca, ci sembra chiaro che la sua posizione, per quanto eccentrica, sia fortemente ancorata e radicata nel terreno ideologico dell'anarchismo, pertanto ci sentiamo di poter ipotizzare che la sua carta costituzionale non sia che una sorta di tentativo di organizzazione formale della rete di congressi e convenzioni che Malatesta oppone alla Costituente. Ne viene di conseguenza che anche l'idea di Berneri sulla natura e la funzione di una Costituzione sia altrettanto originale e sui generis, senza dubbio diversa rispetto alla definizione scientifica e politologica che conosciamo. Il paradosso e l'eresia di un progetto di carta costituzionale di tipo libertario verrebbero così a essere solo apparenti, ma andiamo con ordine.

\section{Una Costituente "altra"}

È un articolo apparso su «L'Adunata dei Refrattari» il 23 luglio $1932^{454}$ a fornirci una prima, importante chiave interpretativa della riflessione politica berneriana in merito alla possibilità di immaginare una costituente libertaria; un articolo che, sorprendentemente, nessuna ricerca e nessuno studio sin qui condotto sul pensiero dell'anarchico lodigiano ha mai citato o mostrato di aver tenuto in considerazione. Eppure è proprio qui che Berneri parla apertamente per la prima volta della possibilità di immaginare una carta costituzionale che possa condurre a degli esiti

\footnotetext{
${ }^{452}$ E. Malatesta, A proposito di Costituente, «Pensiero e Volontà», I, 15 ottobre 1924.

${ }^{453}$ Ibid.

${ }^{454}$ L'Orso [C. Berneri], La Costituente, «L'Adunata dei Refrattari», XI, 23 luglio 1932.
} 
compiutamente autonomisti e federalisti in senso a-statale; è proprio qui che egli suggerisce ai militanti anarchici, desiderosi di incidere sul processo rivoluzionario e sulla futura Italia liberata, di impegnarsi per favorire questo processo. Inoltre, ancora una volta, la sua riflessione politica prende le mosse da una sua originale interpretazione di uno spunto salveminiano, che si conferma essere un punto di riferimento per lui imprescindibile:

Che cos'è la Costituente? Salvemini la definiva sulla sua Unità: "quella cosa che si riunisce in un paese quando questo paese ha mandato a spasso il Governo antico ed ha bisogno di costituirsi un governo nuovo".

Secondo questa definizione, la Costituente sarebbe l'atto costitutivo di un governo in un paese che ne è rimasto senza; atto plebiscitario dunque, di natura statale come scopo ma di natura extra-statale, cioè interamente democratico, come processo ${ }^{455}$.

La citazione riportata da Berneri è estrapolata da un passaggio di un articolo salveminiano del 1919 che vale la pena riportare per intero:

La Costituente è quella cosa che si riunisce in un paese, quando questo paese ha mandato a spasso il Governo antico e ha bisogno di un Governo nuovo. Primo atto, rivoluzione; secondo atto, costituente; terzo atto, che Dio ce la mandi buona ${ }^{456}$.

Non sarà superfluo sottolineare che, ancora una volta, l'anarchico lodigiano sta rileggendo e reinterpretando Salvemini in chiave anarchica e rivoluzionaria: il dibattito che stava allora aprendosi sulle pagine de «L'Unità» avveniva in un contesto politico ben preciso, a Grande Guerra conclusa e con l'aprirsi di una serie di assemblee costituenti negli oramai ex-Imperi centrali - Salvemini cita in proposito il caso tedesco e quello austriaco, ma non manca di indicare anche quello russo, nonostante la rivoluzione bolscevica avesse poi impresso ben altro corso agli eventi. Nell'Italia del dopoguerra, osservava il professore pugliese, si chiede a gran voce una Costituente, si invoca in alcuni casi la repubblica; Salvemini suggeriva allora di partire da alcune riforme a monte di queste richieste (collegio plurinominale e rappresentanza proporzionale) per favorire la formazione di una nuova Camera in grado di lavorare alla riforma dello Stato. Nulla di rivoluzionario, come si può ben vedere, nulla di più lontano dall'interpretazione berneriana sopra evidenziata in corsivo $^{457}$. È probabile che si tratti dell'ennesima e personalissima operazione di filtraggio compiuta da Berneri a pratire dalle parole del professore pugliese, l'ennesima declinazione in chiave rivoluzionaria di spunti provenienti dal periodico riformista, ma non è da escludere che il lodigiano sia stato suggestinonato da un

\footnotetext{
${ }^{455}$ Ibid. I corsivi sono nostri.

${ }^{456}$ [G. Salvemini], La Costituente, «L'Unità», VIII, 2 gennaio 1919.

${ }^{457}$ Due numeri dopo, rispondendo alla lettera di un lettore sul tema della Costituente, Salvemini dichiara: «Nell'Italia di oggi una rivoluzione non è avvenuta. Noi non la desideriamo». Vedi L'Unità [G. Salvemini], Postilla a Un insegnante, La Costituente, «L'Unità», VIII, 23 gennaio 1919.
} 
altro articolo comparso a inizio 1919 su «L'Unità», firmato però dal collaboratore Giovan Battista Klein. Quest'ultimo vi aveva sostentuo che, nella storia, le assemblee costituenti fossero sorte da rivoluzioni intenzionate a fare tabula rasa delle istituzioni precedenti, ma che i tempi moderni non rendono tale passaggio necessario:

Sono le forze reali, in continuo mutamento, di un popolo, che condizionano giorno per giorno il funzionamento delle istituzioni politiche. [...] La carta non impedisce il movimento; e il movimento non si preoccupa della carta ${ }^{458}$.

In altre parole, una carta costituzionale non cristallizza la vita politica e sociale di un popolo, che può continuamente superare e rivedere i principi fondativi del suo Stato. Un discorso che, declinato in chiave libertaria, dà la possibilità di vedere un documento come la Costituzione e il processo della Costituente sotto tutto un altro punto di vista.

Tornando all'articolo di Berneri, l'idea per cui la Costituente sarebbe un «atto plebiscitario» e «interamente democratico, come processo» è una lettura certo originale, ma è anche significativa nel suo cercare di aprire una prospettiva nuova, in cui anche l'eventuale elaborazione di una Costituzione possa diventare il frutto di un percorso di democrazia integrale, un prodotto che sorge e si forma nel contesto di una vasta rivoluzione sociale. Un prodotto destinato a non rimanere fisso e a non intrappolare la società, ma anzi passibile di essere condizionato dallo sviluppo di quest'ultima. Come al solito, l'attenzione dell'anarchico lodigiano è rivolta in prima istanza alla situazione italiana e su quelli che potrebbero essere gli sbocchi di un'insurrezione contro il regime fascista; Berneri ne individua due:

una sommossa antifascista rovesciante la dittatura e lasciante il posto ad un governo provvisorio; una vasta e profonda rivoluzione sociale creante una situazione tale in cui sia impossibile il formarsi di un qualunque governo provvisorio ${ }^{459}$.

Inutile dire che gli anarchici dovrebbero porsi senza alcun dubbio contro la prima opzione, mentre dovrebbero, secondo il parere di Berneri, partecipare attivamente alla seconda, anche perché non avrebbero troppa scelta:

la Costituente è l'unica forma di intesa nazionale per la costruzione del nuovo ordine sociale, e noi non potremo che porci il problema della partecipazione o dell'astensione, sia come votanti sia come rappresentanti di organismi economici, o come delegati con potere strettamente esecutivo ${ }^{460}$.

Insomma, se la nuova costituente originasse direttamente "dal basso" e come esito di una rivoluzione sociale di carattere libertario, tale da rendere superflua la

\footnotetext{
${ }^{458}$ G. B. Klein, La Costituente, «L'Unità», VIII, 18 gennaio 1919.

${ }^{459}$ L'Orso [C. Berneri], La Costituente cit.

${ }^{460}$ Ibid.
} 
presenza dello Stato, gli anarchici non dovrebbero essere spettatori, ma agenti della storia. È un'idea eccentrica rispetto al pensiero anarchico quella berneriana, un'idea che sostanzialmente sovrappone i termini di costituente e di rivoluzione sociale e che ha, come estrema conseguenza, la loro identificazione: abbiamo visto più volte come Berneri non abbia fiducia nello spontaneismo delle masse o nello spirito collettivo, per cui anche la nuova organizzazione sociale dovrà avere i suoi principi fondativi, le sue regole e le sue forme di "autorità"; regole e autorità che avranno certo natura eminentemente diversa da quella di senso comune, in ragione del loro sorgere direttamente dalla società attraverso processi di democrazia integrale -0 , per dirla bernerianamente, di auto-democrazia - favoriti dalla presenza di organismi di autogoverno e autogestione dotati delle più ampie autonomie.

Il quadro è chiaro per l'anarchico lodigiano, ma la sua riflessione politica sta assumendo un posizionamento che non può essere compreso dalla maggior parte del movimento se esso non si convince - come ha sempre suggerito Berneri - ad aggiornare, approfondire e studiare una propria tattica rivoluzionaria, mettendo da parte quello che egli qualifica come «dilettantismo culturale» ${ }^{461}$. Sono esortazioni che oramai conosciamo bene, ma val la pena di tornare a leggerle ancora una volta:

I tempi richiedono una nostra mobilitazione culturale. Vi è il mito bolscevico da sventare. Vi è il sistema capitalistico in istato fallimentare da anatomizzare. Vi sono i problemi della rivoluzione da discutere. Vi sono gli equivoci social-democratici da mandare in aria. E tante altre battaglie da combattere. [...] E la nostra stampa, che deve contribuire a rovesciare il fascismo e a creare quelle correnti di idee e di sentimenti che evitano gli errori e gli aborti che le recenti rivoluzioni hanno mostrati, deve essere all'altezza del compito ${ }^{462}$.

\section{Il complesso dibattito nel movimento anarchico}

Le idee di Berneri circa il programma minimo hanno difficoltà a far breccia in un movimento, in cui predomina ancora l'attaccamento integrale al programma massimo e all'idea che la dissoluzione dello Stato condurrà spontaneamente e inevitabilmente a una libera organizzazione federale. L'anarchico lodigiano però, lo abbiamo visto, deve anche impugnare la penna e battagliare contro quanti nello stesso movimento sono giunti a sostenere la partecipazione a governi provvisori di tipo rivoluzionario se non alla conquista vera e propria del predominio politico, pur di rompere il proprio isolamento e di realizzare qualcuno dei propri postulati.

Altamente significativo è allora il dibattito che si sviluppa tra il settembre e l'ottobre del 1932 sulle colonne de «L'Adunata dei Refrattari», in cui Berneri ha l'opportunità di qualificare con nettezza la propria posizione, scontrandosi però contro il muro dell'intransigentismo anarchico. La discussione ha origine da un commento che il lodigiano fa al libro Preanarchia ${ }^{463}$, pubblicato in quell'anno da

${ }^{461}$ C. Berneri, Il dilettantismo culturale, «L'Adunata dei Refrattari», XI, 6 agosto 1932.

${ }^{462}$ Ibid.

${ }^{463}$ C. Berneri, La pre-anarchia, «L'Adunata dei Refrattari», XI, 17 settembre 1932. 
Randolfo Vella sotto lo pseudonimo di Uno della tribù ${ }^{464}$, esprimendo le sue perplessità circa gli intenti realizzatori dell'autore. Berneri ribadisce:

Io non concepisco la vittoria degli anarchici nella rivoluzione come predominio politico, bensì come impossibilità di qualsiasi dittatura politica non solo, ma anche dell'affermarsi di un ordine sociale in cui, pur non essendo soppresso l'antagonismo tra $\mathrm{i}$ partiti, prevalga un sistema di rappresentanze di carattere esecutivo prevalentemente tecnico ${ }^{465}$.

E più avanti precisa:

La nostra arma non è in quella tattica demagogica preconizzata da "Uno della tribù", bensì dall'affermazione chiara ed energica di un integrale soviettismo, nel quale confluiscano, temprandosi e completandosi, tutte le correnti socialiste del popolo e tutte le rappresentanze dei partiti d'avanguardia. [...]

In questa negazione della dittatura politica di qualsiasi partito, gli anarchici possono affermarsi politicamente, non come forza di predominio egemonico, ma come forza di equilibrio e di potenziamento.

La vittoria sarà nostra a questa condizione e sarà tanto più nostra quanto meno sarà appariscente la nostra partecipazione agli organismi direttivi del nuovo ordine sociale $^{466}$.

Eccolo, ancora una volta, il concretismo rivoluzionario di Berneri. Fatta tabula rasa di quanto vi è di inattuale e di astratto nelle idee politiche anarchiche, al movimento non resta che elaborare una tattica che lo porti a giocare un ruolo di primo piano nella rivoluzione antifascista in modo tale da evitare la vittoria di movimenti o partiti intenzionati a conquistare il potere politico e a mettere in atto nuove soluzioni accentratrici, in modo tale da favorire un contemporaneo sviluppo della rivoluzione stessa in termini integralmente sociali e federalisti, il cui cuore pulserà nelle autonomie degli organismi di autogoverno. Berneri lo definisce «soviettismo» e sappiamo che l'esperienza rivoluzionaria dei soviet aveva destato il suo interesse sin dal 1918-19, quando inizia a sbozzare le sue idee di un sistema di auto-democrazia capace di consentire al massimo il coinvolgimento e la partecipazione dei cittadini all'amministrazione e alle decisioni politiche. Di nuovo,

${ }^{464}$ Uno della tribù [R. Vella], Preanarchia: pareri pratici sull'organizzazione della società preanarchica, Edizioni Vogliamo, Lugano, 1932.

Randolfo Vella (1893-1963) è stato un militante anarchico. Il suo pseudonimo viene dalla sua stessa famiglia, nota negli ambienti libertari come "tribù Vella": Randolfo è uno degli otto figli dei Vella, tutti schedati come anarchici e protagonisti della scena politica di Milano. Espatriato in Svizzera nel 1928, vi fonda il mensile "Vogliamo!», collabora alla stampa anarchica internazionale e ricopre ruoli di primo piano tra gli esuli anarchici. Vedi «Vella, Randolfo», in M. Antonioli et al. (diretto da), Dizionario biografico degli anarchici italiani, vol. II cit., pp. 663-666.

${ }^{465} \mathrm{C}$. Berneri, La pre-anarchia cit.

${ }^{466}$ Ibid. Il corsivo è nostro. 
siamo di fronte a un'interpretazione personale data dal lodigiano sulle possibili applicazioni libertarie degli organismi di base sorti durante la Rivoluzione russa, non certo a una fiducia o a un'esaltazione degli sviluppi del sistema sovietico realizzatosi nell'URSS; eppure al suo articolo segue una critica serrata da parte di Max Sartin, pseudonimo di Raffaele Schiavina ${ }^{467}$.

Secondo Sartin, il soviet è «un organo elettivo avente potere legislativo» e gli anarchici dovrebbero essere

recisamente avversi ai corpi elettivi aventi giurisdizione politica sulle città, o sul villaggio, nella regione o sulla nazione [...].

La nostra opposizione non è dettata da capriccio, ma dalla nostra qualità di anarchici, cioè nemici irriducibili di ogni e qualsiasi potere politico e della coscienza che cesseremmo di essere anarchici il momento in cui da questa opposizione desistessimo ${ }^{468}$.

Sartin non prende neanche in esame la possibilità che il soviet possa svilupparsi in senso libertario, sostenendo che la natura «autoritaria del Soviet era completa sin dall'inizio sia per il carattere rappresentativo della sua costituzione, sia per gli scopi governativi della sua funzione». ${ }^{469}$ L'autore sostiene altresì che gli anarchici

hanno difeso [...] le autonomie sovietiche in generale e quelle dei soviet di Kronstadt in particolare, per la stessa ragione che difendono le autonomie locali e individuali contro le rappresaglie feroci e gli assorbimenti dittatoriali dei regimi borghesi. [...]

Ma da questo, che è compito elementare di ogni avanguardia di progresso ad assegnarsi come meta il Sovietismo [...] corre un abisso insormontabile.

La nostra meta è sempre l'Anarchia, a cui non vi conduce certamente il Soviet, come non vi conduce la Costituente ${ }^{470}$.

Con l'ultima sentenza del suo intervento, ci sembra evidente che Sartin abbia fornito una stroncatura non solo dell'ipotesi sovietista di Berneri, ma anche di quella sua ipotesi di Costituente come esito di un'ampia rivoluzione sociale organizzata secondo un sistema federalista e autonomista. Non ultimo, Sartin mostra uno spiccato integralismo anarchico nel suo dirsi recisamente contrario a qualunque ipotesi organizzativa che contempli la presenza di organismi rappresentativi. In altre

${ }^{467}$ M. S. [M. Sartin], Sovietismo, «L'Adunata dei Refrattari», XI, 17 settembre 1932.

Raffaele Schiavina (1894-1987) è stato un importante militante e pubblicista anarchico di tendenza antiorganizzatrice. Emigrato negli USA nel 1913, è qui protagonista di numerose esperienze editoriali, tra cui sono da ricordare la collaborazione a «Cronaca Sovversiva» e a «L'Adunata dei Refrattari»; di quest'ultima sarà direttore a partire dal 1928 e fino al 1971, anno in cui la testata chiude i battenti. Vedi «Schiavina, Raffaele», in M. Antonioli et al. (diretto da), Dizionario biografico degli anarchici italiani, vol. II cit., pp. 516-521.

${ }^{468}$ Ibid.

${ }^{469}$ Ibid.

${ }^{470}$ Ibid. 
parole, una contrapposizione frontale alle idee berneriane che non tarderà a svilupparsi in uno scambio di posizioni maggiormente articolato.

\section{La polemica Berneri-Sartin sui soviet}

Berneri non è certo un ingenuo e, nell'articolo Sovietismo, anarchismo e anarchia con cui risponde a Max $\operatorname{Sartin}^{471}$, si dichiara consapevole che il soviet possa «contenere in nuce le tendenze alla cristallizzazione statale», ma sostiene che esso rappresenti anche

l'immediata ed inevitabile espressione del bisogno delle masse di darsi un sistema di coordinazioni capace di assicurare e possibilmente aumentare e migliorare il tenore di vita, la difesa delle posizioni conquistate, la sostituzione degli organi e delle funzioni rispondenti ai generali bisogni ${ }^{472}$.

Il soviet sarebbe insomma per Berneri un elemento imprescindibile in un ipotetico programma minimo degli anarchici che intenda presentarsi come un'alternativa di organizzazione politica e sociale credibile, applicabile e concreta. È un corpo politico che può aprire la strada per procedere verso l'anarchia, sempre che gli anarchici si convincano di questo e si impegnino per «conservare al Soviettismo quanto vi è in esso di autonomia, di anti-Stato, di ex-lege, cercando che il sistema sia sano alle radici e saldo nei suoi ulteriori sviluppi» ${ }^{473}$.

Berneri prosegue nel suo intervento rilanciando il suo approccio problemista e la sua posizione concretista rivoluzionaria:

I problemi della rivoluzione sono quelli che sono, risolvibili nel quadro di una maturità politica e morale di un dato complesso di economici fattori obiettivi che impongono soluzioni non solo immediate ma generali. Un organismo qual è lo Stato odierno può essere demolito, ma alla sua ossatura fa riscontro tutto quel sistema di fasci muscolari e nervosi, che sono i servizi pubblici. Questi vanno riorganizzati, ed essendo [...] degli organismi eminentemente nazionali, al di sopra del villaggio, della città, della regione dovrà pulsare un sistema di centri direttivi $[\ldots]^{474}$.

Berneri ammette che la soluzione sovietista non coincida interamente con quella anarchica, tuttavia la ritiene più un'opportunità che non un ostacolo. Proseguendo, il lodigiano si profonde in quella che è con ogni probabilità una summa tra le più significative e sintetiche del suo pensiero politico; egli afferma che

\footnotetext{
${ }^{471}$ C. Berneri, Sovietismo, anarchismo e anarchia, «L'Adunata dei Refrattari», XI, 15 ottobre 1932, segue nota di m. s. [M. Sartin]. L'articolo e la nota sono riprodotti in Id., Pietrogrado 1917 Barcellona 1937 cit., pp. 118-127 (col titolo Il soviet e l'anarchia), e in Id., Scritti scelti cit., pp. 130-138.

${ }^{472}$ Ibid.

${ }^{473}$ Ibid.

${ }^{474}$ C. Berneri, Sovietismo, anarchismo e anarchia cit.
} 
l'anarchismo è il viandante, che va per le vie della storia, lotta con gli uomini quali sono e costruisce con le pietre che gli fornisce la sua epoca. [...] Egli sa che il destino, che la sua missione è di riprendere il cammino, additando alle genti nuove mete. Ma quando il popolo insorto dai rottami dello Stato fa materiale per costruire il libero Comune, e contro la Banca e il Consorzio padronale erge il Sindacato, e nella palestra del Comune si addestra ad amministrare, l'anarchico comprende che nella storia si agisce sapendo di essere popolo per quel tanto che permetta di essere compresi e di agire, additando mete immediate, interpretando reali e generali bisogni, rispondendo a sentimenti vivi e comuni.

Recisamente contrari al Sovietismo noi? Noi che nelle autonomie locali avremo la migliore trincea per sbarrare la strada allo Stato? Noi che non possiamo sognare di veder realizzata l'Anarchia se non dopo la più larga e la più profonda esperienza di auto-democrazia nel campo dell'amministrazione cooperativa e comunale ${ }^{475}$ ?

\title{
In definitiva, per Berneri
}

Il sovietismo è il sistema di auto-amministrazione popolare rispondente ai bisogni fondamentali della popolazione, rimasta priva degli organismi amministrativi statali. Questo sistema può permettere la ripresa della vita economica, compromessa dal caos insurrezionale, e può servire di base alla fondazione di un nuovo ordine sociale, costituendo inoltre una proficua palestra di auto-amministrazione preparante il popolo a sistemi di maggiore autonomia ${ }^{476}$.

Per dare finalmente inizio alla tanto desiderata discussione sui problemi della rivoluzione, il lodigiano lancia un invito al suo interlocutore:

M. S. dovrebbe, per convincermi che ho torto, spiegarmi quale sistema crede possibile possa sprigionare la rivoluzione italiana e con quali linee programmatiche e tattiche l'anarchismo italiano potrebbe agire in seno a quella rivoluzione raggiungendo i suoi obiettivi possibili $[\ldots]^{477}$.

La risposta di Max Sartin non cede di un millimetro rispetto a quella di partenza, a partire dal fatto di contestare a Berneri di intendere col termine sovietismo «cosa diversa da ciò che tutti gli altri intendono, cosa che non è mai esistita fuorché nel suo desiderio e nella sua immaginazione $\gg{ }^{478}$. È soprattutto l'affermazione che l'anarchismo debba accettare compromessi tra l'idea e i fatti però a ripugnare all'interlocutore del lodigiano:

La parola compromesso è una brutta parola, ciò che implica è ancora più brutto. In ogni modo, se si è costretti a subire il compromesso, non si deve accettarlo mai. Il fatto della vita sociale impone delle restrizioni e degli ostacoli all'applicazione integrale dell'Idea oggi, e forse anche domani. Ma noi non possiamo considerare

\author{
${ }^{475}$ Ibid. \\ ${ }^{476}$ Ibid. \\ ${ }^{477}$ Ibid. \\ ${ }^{478}$ Nota di m. s. [M. Sartin] a C. Berneri, Sovietismo, anarchismo e anarchia cit.
}


quelle restrizioni come un passo verso l'Anarchia, quando invece ne sono una barriera: né possiamo accettare come definitivi quegli ostacoli. [...]

Ora, io penso che gli anarchici non debbano essere, neanche transitoriamente, fautori di istituzioni autoritarie ${ }^{479}$.

Sartin dichiara inoltre di avere presenti

le complicazioni della vita moderna: penso anzi che i cosiddetti servizi pubblici, che tanto preoccupano il compagno Berneri, saranno assai più numerosi e complicati in seguito all'espropriazione della terra e di tutti i mezzi di produzione e di scambio. [...] Né intendo che l'Anarchia significhi ritorno alla vita semplice o primitiva dei campi e del villaggio al crepuscolo della civiltà, bensì superamento, integrazione, su tutti i campi dell'umana attività delle conquiste sinora raggiunte ${ }^{480}$.

La fiducia di Sartin nello spontaneismo, tanto avversato da Berneri, appare in questo passaggio con grande chiarezza, e da solo basterebbe per far comprendere quanto il dialogo con parte cospicua del movimento anarchico dovesse essere difficile e complessa per il lodigiano. Lo sviluppo dell'argomentazione di Max Sartin non fa che confermare quanta incomunicabilità potesse esserci tra una posizione problemista e concretista come quella di Berneri e quella di chi sosteneva che l'anarchismo dovesse, in definitiva, «risolvere anarchicamente il problema della gestione diretta del patrimonio sociale», pena il contentarsi di «espedienti autoritari» che non solo allontanerebbero l'avvento dell'anarchia, ma addirittura renderebbero l'anarchismo «un pleonasmo assurdo che non serve ad altro che a designare una delle tante correnti autoritarie del movimento rivoluzionario» ${ }^{481}$.

In conclusione, Sartin si trova a dover fare un'ammissione che riteniamo significativa per comprendere le difficoltà che si paravano di fronte a Berneri nel suo tentativo di convincere il suo movimento ad aggiornare la propria battaglia politica:

non ho un programma politico da offrire all'anarchismo italiano pei suoi bisogni cumulativi in occasione della prossima rivoluzione.

Non ho che una convinzione, e cioè che le deviazioni, le transazioni autoritarie dell'anarchismo, siano al tempo stesso dannose all'Anarchia, al popolo italiano, alla sua rivoluzione.

Ed un proposito: mescolarmi tra la folla dei diseredati che non aspirano a creare per sé nuovi monopoli o privilegi, che sperano con la rivoluzione conquistarsi il pane e la libertà, viverne la passione, combatterne le battaglie per l'abolizione di tutte le ingiustizie. [...]

È poca cosa, ma mi sembra ancor meglio del sovietismo ${ }^{482}$.

\footnotetext{
${ }^{479}$ Nota di m. s. [M. Sartin] a C. Berneri, Sovietismo, anarchismo e anarchia cit.

${ }^{480} \mathrm{Ibid}$.

${ }^{481}$ Ibid.

${ }^{482}$ Ibid.
} 
Questo ostinato barricarsi nella purezza e nell'intransigenza delle proprie idee da parte di Max Sartin - e, c'è da crederlo, di gran parte del movimento anarchico sono ciò contro cui Berneri ha sempre lottato a partire dal 1919 sulla scorta dell'influenza salveminiana e dell'ampio dibattito politico e culturale svoltosi in Italia tra primo dopoguerra e trionfo della dittatura fascista.

\section{Soviet, un perno del programma}

Non c'è da stupirsi che il lodigiano abbia scelto di non proseguire lo scambio, anche perché deve essergli parso evidente che scambio non poteva esserci e che sarebbe stato tanto meglio profondere le proprie energie in nuove elaborazioni programmatiche e nella ricerca di nuove convergenze tattiche. Non per questo rinuncia a cercare di convincere il movimento anarchico della possibilità di dare al sovietismo un'interpretazione libertaria, condizione necessaria per persuadere i suoi a farne fonte di studio e di ispirazione per quell'idea di Costituente "altra" che aveva iniziato ad attraversargli la mente.

Il 10 novembre 1932 compare sulla rinata «Umanità Nova» un articolodi Berneri intitolato Soviettismo e bolscevismo da cui vale la pena trarre questa citazione, a sua volta estratta dal noto studio di Luigi Fabbri Dittatura e rivoluzione (1921):

I sovieti sorsero, nella rivoluzione russa del 1917, indipendentemente dal partito bolscevico. Furono un'organizzazione spontanea, autonoma, federale, rispondente al bisogno di regolare la produzione e gli scambi, di organizzare la difesa della rivoluzione, ecc. [...]

I bolscevichi si impadronirono dei sovieti contro lo spirito e la pratica del Sovietismo $^{483}$.

Più precisamente, Fabbri si era espresso così:

I sovieti sono sorti in realtà indipendentemente dal bolscevismo. Essi scaturirono dallo spirito di iniziativa degli operai delle città e dei villaggi, spronato dal bisogno di provvedere immediatamente in modo organico alle necessità pratiche della rivoluzione, alle relazioni, all'alimentazione delle masse, alla produzione, all'armamento, ecc. Avevano una organizzazione semplice, federalistica o autonomista che dir si voglia, pur essendo ciascuno in rapporto con gli altri per le necessità della vita sociale nel proprio villaggio, o rione, o città. L'intesa fra $i$ vari sovieti avveniva su basi egualitarie e senza coercizioni degli uni sugli altri.

[...] Insomma, i sovieti si devono più che altro alle tendenze anarchiche della masse russe; e se i bolscevichi sono riusciti a trasformarli in loro organismi di governo, questo non toglie che l'idea soviettista, antiautoritaria e federalista, contradica e cozzi contro lo spirito autoritario ed accentratore del bolscevismo, e cioè della concezione socialdemocratica e marxista della rivoluzione ${ }^{484}$.

${ }^{483}$ [C. Berneri], Soviettismo e bolscevismo, «Umanità Nova», I, 10 novembre 1932.

${ }^{484}$ L. Fabbri, Dittatura e rivoluzione, Libreria editrice internazionale Giovanni Bitelli, Ancona, 1921, pp. 65-67. Il corsivo è originale. 
Il tema del sovietismo, come è stato giustamente rilevato da Carrozza, costituisce ormai «uno degli assi portanti del pensiero politico berneriano» ${ }^{485} \mathrm{e}$ risulta essere, soprattutto nel corso dagli anni '30, un elemento sempre più fortemente presente nella sua riflessione. Ne abbiamo una chiara dimostrazione in una lettera che Berneri scrive a Carlo Frigerio nel febbraio 1933, dicendogli:

ho tenuto giorni or sono, per invito della LIDU di Sartrouville, una conferenza: Bolscevismo, Sovietismo e Anarchismo - che ha avuto discreto successo (non in applausi ma in...commozioni cerebrali, che più contano). [...]

Lo schema della conferenza: Bolscevismo, Sovietismo e Anarchismo - è questo:

$1^{\circ}$ Il marxismo e l'estinzione dello Stato

$2^{\circ}$ Lo Stato e le classi nell'U.R.S.S.

$3^{\circ}$ La distruzione dello Stato borghese

$4^{\circ}$ Dittatura del proletariato e socialismo di Stato

$5^{\circ}$ La critica anarchica della «dittatura del proletariato»

$6^{\circ}$ Democrazia e dittatura

$7^{\circ}$ L'origine del Soviet in Russia

$8^{\circ}$ Gli anarchici russi e il sovietismo

$9^{\circ}$ Il Sovietismo e la democrazia operaia russa

$10^{\circ}$ La lotta tra bolscevichi e anarchici: (dal '18 - al '21)

$11^{\circ}$ La Comune di Kronstadt e quella di Parigi

$12^{\circ}$ Dal Leninismo allo Stalinismo

$13^{\circ}$ Gli anarchici italiani, tedeschi e ungheresi di fronte al Sovietismo ${ }^{486}$

Come si può vedere, la scaletta della conferenza è ricca e nutrita, e mostra tutti i segni di una riflessione strutturata che è senza dubbio tesa a sostenere e ad argomentare una differenza tra interpretazione bolscevica e interpretazione anarchica degli organismi consiliari del soviet. Alla luce di quanto abbiamo fin qui esposto, ci sembra però ragionevole ritenere che non solo Berneri stesse insistendo sull'ipotesi sovietista integrandola nel proprio pensiero politico, ma anche che egli intendesse farne una cellula-base del programma federalista libertario: nella sua originale ipotesi di una possibile Costituente "altra", sembra ormai possibile intuire

${ }^{485}$ G. Carrozza, Il "sovietismo" di Camillo Berneri, in Camillo Berneri, singolare/plurale. Atti della giornata di studi, Reggio Emilia, 28 maggio 2005, Edizioni Biblioteca Panizzi e Archivio Famiglia Berneri-Aurelio Chessa, Reggio Emilia, 2007, p. 55.

${ }^{486}$ Lettera di Camillo Berneri a Carlo Frigerio (s. 1., s. d., ma Parigi, febbraio 1933), in C. Berneri, Epistolario inedito, vol. II, a cura di Paola Feri e Luigi Di Lembo, Edizioni Archivio Famiglia Berneri, Pistoia, 1984, pp. 86-87.

Carlo Frigerio (1878-1966) è stato un importante pubblicista anarchico. Svizzero di nascita e conoscitore di molte lingue, è parte del gruppo promotore de «Le Réveil/Il Risveglio». In contatto con i principali esponenti dell'anarchismo internazionale (Malatesta, Bertoni, Goldmann, Shapiro), sarà in seguito tra i redattori più importanti di «Umanità Nova» e di «Pensiero e Volontà», nonché curatore della pubblicazione dell'«Almanacco libertario pro vittime politiche». 
due coordinate principali, costituite dalle autonomie politico-territoriali (Comune) e da quelle sociali (consigli, sindacati, cooperative, etc.); in altre parole, soprattutto riflettendo sulle recenti esperienze rivoluzionarie che egli aveva visto attraversare l'Europa e l'Italia nell'immediato dopoguerra, Berneri sta con ogni probabilità iniziando a ipotizzare che l'approssimazione storica all'anarchia potrebbe essere costituita da una sorta di Repubblica dei consigli organizzata, ovviamente, secondo criteri federalisti e libertari.

Un programma minimo del genere avrebbe potuto riscuotere l'interesse di numerose forze politiche, distanti sia dalle tendenze comuniste sia da quelle della Concentrazione antifascista; avrebbe potuto fornire una piattaforma su cui farle convergere e attraverso cui sviluppare un'unità d'azione in cui il movimento anarchico avrebbe potuto - se si fosse rivelato all'altezza - ricoprire un ruolo di riferimento. Restavano da individuare i soggetti più adatti per tale alleanza e da elaborare un programma che fosse, per quanto minimo, il più vasto, profondo e concreto possibile. Le cose sembreranno evolversi favorevolmente nel giro di pochi anni.

\section{Il complesso avvicinamento tra anarchici e giellisti}

Luigi Fabbri nel 1933 stava assumendo un atteggiamento di apertura verso il movimento di «Giustizia e Libertà»: è interessante ai fini di questa ricerca leggere un suo intervento nei "Quaderni di Giustizia e Libertà»" ${ }^{487}$, soprattutto considerando che proprio lui era stato tra le voci contrarie alla tattica rivoluzionaria suggerita da Berneri. Fabbri fa mostra di apprezzare l'azionismo e lo slancio di GL, elementi ritenuti potenzialmente di primo piano nella rivoluzione che verrà:

Ciò che soprattutto approvo in essa è l'idea che la rivoluzione debba procedere immediatamente, fin dai suoi primi passi, [...] a realizzazioni pratiche di demolizione, espropriazione e riorganizzazione che possano restare conquista acquisita del popolo italiano $[\ldots]^{488}$.

Il carattere spontaneo, il rifiuto del dirigismo centralizzatore e la prospettiva di coordinamento e aiuto reciproco a livello pratico sono altri elementi apprezzati dal Fabbri. Il punto debole, a suo dire, è di non essersi limitati al ruolo movimentistico, dandosi un programma ritenuto troppo vincolante e che appare una «via aperta ad una involuzione in senso democratico borghese». Tuttavia egli è convinto che $\mathrm{i}$ programmi nella rivoluzione varranno solo come suggerimento sperimentale, dunque non insiste troppo su questo punto, registrando invece come GL rappresenti un progresso rispetto alle tendenze antifasciste socialdemocratiche e riformiste, e sostenendo che

\footnotetext{
${ }^{487}$ L. Fabbri, Qualche risposta all'inchiesta di «G.L.», «Quaderni di Giustizia e Libertà», n 7 , giugno 1933, pp. 111-116.

${ }^{488}$ Ivi, p. 111.
} 
l'atteggiamento di fronte a "G. e L." qual è presentemente, di tutti coloro che non ne condividono in tutto o in parte il programma, o che non vi aderiscono perché militanti [in] altri campi rivoluzionari, dovrebbe secondo me [...] essere un atteggiamento di cordialità "dal di fuori” ${ }^{489}$.

Dunque la simpatia per il movimento giellista ha già superato parte delle remore iniziali, anche perché abbiamo visto come in questo periodo per gli anarchici sia importante trovare interlocutori per rompere il proprio isolamento. Giacché tanto il movimento giellista quanto quello anarchico si oppongono ai «partigiani della dittatura», si intuisce la possibilità di stabilire un dialogo costruttivo, pur rimarcando che gli anarchici tenderanno sempre a combattere al di fuori dei parlamenti, per l'indebolirsi dello Stato e per la diffusione delle autonomie e delle libertà fino al massimo possibile.

Urge ancora, secondo Fabbri, una chiarificazione che elimini ogni possibile equivoco da parte di GL, che certo aspira a rappresentare il futuro governo democratico, nei confronti delle forze d'opposizione extraparlamentari:

Poiché gli anarchici non vogliono comandare agli altri né costringere, neppure a fin di bene, alcuno a fare quel che essi vogliono, la loro posizione non lascia luogo ad equivoci né ha bisogno di troppe spiegazioni. Maggiori spiegazioni invece, secondo me, debbono darle tutti coloro che in un modo o nell'altro vogliono andare al potere, specialmente coloro che ripudiano i sistemi dittatoriali e pongono la candidatura di governanti per gli uomini del loro partito in nome della libertà ${ }^{490}$

Riserve a parte, sarà soprattutto a partire dal 1934 che l'atteggiamento degli anarchici verso i giellisti muterà sensibilmente, allorché GL abbandonerà la piattaforma concentrazionista accentuando le proprie specifiche caratteristiche. Tra queste certo vanno ricordate le idee consiliariste e di pluralismo democratico di derivazione gobettiana sostenute dal gruppo giellista torinese (composto, tra gli altri, da Ginzburg, Levi, Foa e Giua) e la posizione socialista e tendenzialmente antiautoritaria dell'animatore Rosselli, che avversava tanto il marxismo quanto il liberalismo tradizionale, ponendo il proprio movimento in una posizione quanto meno di prossimità rispetto alla filosofia politica libertaria, col recupero di temi e contenuti proudhoniani, bakuniniani e federalisti-risorgimentali ${ }^{491}$. Emblematico a tal proposito sarà il celebre editoriale rosselliano Contro lo Stato (21 settembre $1934)^{492}$ che, a partire da una netta contrapposizione tra Stato e società, propone di far risorgere quest'ultima sotto forma di «federazione di associazioni quanto più libere e varie possibili», dichiarando di ricollegarsi «alla tradizione rivoluzionaria

\footnotetext{
${ }^{489}$ L. Fabbri, Qualche risposta all'inchiesta di «G.L.» cit., p. 114.

${ }^{490}$ Ivi, pp. $115-116$.

${ }^{491}$ Vedi su questi aspetti C. Malandrino, Socialismo e libertà. Autonomie, federalismo, Europa da Rosselli a Silone, Franco Angeli, Milano, 1990, soprattutto le pp. 109-129, e S. Fedele, Il retaggio dell'esilio cit., pp. 103-106.

${ }^{492}$ G.L. [C. Rosselli], Contro lo Stato, «Giustizia e Libertà», I, 21 settembre 1934.
} 
europea, a Proudhon, a Bakounine, allo stesso Marx» ${ }^{493}$. Infine va sottolineata l'influenza esercitata sul movimento giellista dal sociologo russo Gurvitch, che di fatto è il principale tramite attraverso cui avviene il recupero del pensiero politico di Proudhon e si sviluppa il conseguente dibattito intorno al tema del federalismo all'interno dei «Quaderni di Giustizia e Libertà» ${ }^{494}$.

Gli apprezzamenti e l'interesse da parte anarchica si faranno da questo momento sempre più vivaci: a conferma di ciò si può citare una lettera di Luigi Fabbri a Carlo Rosselli del 14 ottobre 1934, che abbiamo potuto consultare negli Archivi di Giustizia e Libertà conservati presso l'Istituto Storico della Resistenza in Toscana e che solo recentemente è stata pubblicata nel voluminoso epistolario di Fabbri ${ }^{495}$. In questa lettera, spedita dall'Uruguay, Fabbri spiega di aver ricevuto il 26 settembre una lettera da Rosselli insieme a un pacco di arretrati del settimanale giellista in cui mancava però la copia del 21 settembre, contenente il famoso articolo Contro lo Stato. Leggendo un trafiletto pubblicato nella rubrica «Stampa amica e nemica» del $\mathrm{n}^{\circ} 15$ di «Giustizia e Libertà» (21 agosto), l'anarchico viene colpito dal fatto che il settimanale giellista, rispondendo a un socialdemocratico tedesco, avesse fatto mostra di prendere le distanze da Bakunin e dall'individualismo. Fabbri aveva dunque maturato l'intenzione di criticare la presa di posizione di GL con un passaggio riportato integralmente nella lettera a Rosselli:

In quanto al ricollegamento col bakuninismo, ora respinto, non ricorda "G. e L." di essersene in qualche modo fatto un vanto nel secondo numero della sua rivista, col riesumare la "Libertà e Giustizia" di Bakunin del $1865^{496}$, approvandone il principio

${ }^{493}$ Ibid.

${ }^{494}$ Vedi G. Manganaro Favaretto, Proudhon in Italia. Una riflessione politica incompresa, Edizioni Università di Trieste, Trieste, 2000 , pp. 88-98, e D. Paci, “Proudhon in esilio". La ricezione del pensiero proudhoniano negli ambienti del fuoruscitismo italiano in Francia (anni Venti e Trenta), «Società e Storia», XXXIV, n 131, gennaio-marzo 2011, soprattutto le pp. 113-121.

Georges Gurvitch (1894-1965) è stato un sociologo russo. Inizia la sua carriera di docente in Russia, che abbandona nel 1920 per trasferirsi prima in Cecoslovacchia e quindi in Francia. Naturalizzato francese nel 1928, risiederà a Parigi fino alla Seconda guerra mondiale, quando espatrierà negli Stati Uniti dove resterà fino al 1946, anno del suo rientro in Francia. È noto soprattutto per aver contribuito alla nascita della sociologia del diritto.

${ }^{495}$ Lettera di Luigi Fabbri a Carlo Rosselli (Montevideo, 14 ottobre 1934), in ISRT, AGL, Fondo C. Rosselli, b. 1, sez. 1; riprodotta in L. Fabbri, Epistolario ai corrispondenti italiani ed esteri (1900-1935), a cura di Roberto Giulianelli, Edizioni Biblioteca Franco Serantini, Pisa, 2005, pp. 491-492. Sulle prossimità e affinità tra Fabbri e Rosselli si è particolarmente soffermata C. Aldrighi, Luigi Fabbri in Uruguay (1929-1935), in R. Giulianelli (a cura di), Luigi Fabbri. Studi e documenti sull'anarchismo tra Otto e Novecento, Edizioni Biblioteca Franco Serantini, Pisa, 2005, specialmente alle pp. 78-92.

${ }^{496}$ Il riferimento è all'articolo di [C. Rosselli e A. Garosci], "Libertà e Giustizia" e "Giustizia e Libertà" , "Quaderni di Giustizia e Libertà», n² 2, marzo 1932, pp. 53-54 che si chiudeva auspicando una soluzione radicale del problema delle autonomie in Italia dichiarando: «ci è piaciuto ricordare 'Libertà e Giustizia' e.... Bakunin». 
di autonomia e la critica allo Stato centralista, che non era individualismo di sicuro, ma che come tale vien gabellato dai marxisti? Ha forse cambiato parere in proposito? Ce ne spiacerebbe ${ }^{497}$.

Fortunatamente, prosegue Fabbri, il successivo arrivo di un nuovo pacco di copie "Giustizia e Libertà» gli ha permesso di leggere e lodare l'editoriale rosselliano Contro lo Stato, dichiarando anzi che esso «è arrivato in tempo per farmi togliere da uno 'spunto critico e polemico' del mio foglio...intermittente un capoverso che avrebbe potuto farmi passare per ingiusto» ${ }^{498}$.

Nonostante ciò, l'esule uruguaiano non nasconde di nutrire comunque qualche riserva in merito all'atteggiamento e alle prospettive politiche di GL:

Di certo la vostra posizione è più vicina alla nostra, dal punto di vista della libertà, di quella dei marxisti. Ma permane la differenza sostanziale, che il vostro è sempre un antistatalismo...di governo, mentre il nostro è contro il governo, anche a quello di domani, nel quale fatalmente le buone intenzioni son condannate a naufragare sotto l'onda dei fatti ${ }^{499}$.

Fabbri si dichiara comunque non contrario a priori in merito a una possibile collaborazione al settimanale «Giustizia e Libertà». Il suo timore è solo sull'opportunità o meno di tale accordo editoriale, temendo che non possa che generare fraintendimento:

È piuttosto questione d'opportunità e di maggiore o minor rendimento, ed anche questione di non aumentare la confusione delle lingue. Eppoi che cosa dire di speciale dalla vostra tribuna? Non saprei. Vedremo. Del resto, anche senza il suo cortese invito, non avrei mancato, né mancherò, di ricorrere alla vostra ospitalità quando ciò mi sembrasse necessario per una qualche ragione $\mathrm{e}^{500}$.

Il fatto che Carlo Rosselli avesse invitato l'anarchico a una collaborazione col giornale della sua sigla è indicativo del fatto che l'apertura tra anarchici e giellisti non fosse unilaterale, bensì reciproca. Ma al di là della collaborazione giornalistica, traspare nella lettera anche uno slancio umano ed emotivo, che è segno di profonda stima e di rispetto intellettuale tra queste due figure:

Anche a me dispiace d'esser così lontano e non conoscerla di persona. Alle volte i rapporti personali e d'amicizia permettono di darsi una mano in qualche cosa di concreto, meglio dei rapporti di partito e al di fuori di questi $^{501}$.

\footnotetext{
${ }^{497}$ Lettera di Luigi Fabbri cit.

${ }^{498}$ Ibid.

${ }^{499}$ Ibid.

${ }^{500}$ Ibid.

${ }^{501}$ Ibid.
} 
Nella parte conclusiva della lettera Fabbri ha un pensiero anche per Nello Rosselli, che conobbe frequentando il Salvemini e che aiutò nella ricerca di alcuni documenti per il suo studio sul rapporto tra Mazzini e Bakunin. Richiede di mandargli, con le dovute prudenze, i suoi saluti, e se fosse possibile avere una copia del suo recente libro su Pisacane, anche solo in prestito. A suggello di quanto emerso, non sarà superfluo ricordare che in «Giustizia e Libertà» del 12 luglio 1935 comparirà un trafiletto per commemorare la scomparsa di Luigi Fabbri avvenuta il 23 giugno di quell'anno.

A partire dal 1934 si può dire dunque che vi siano segnali di prossimità, pur nelle rispettive specificità, tra $i$ due movimenti politici. Il contatto tenderà ad aumentare e a farsi più complesso, intuendo la possibilità di stabilire un'unità d'azione che travalicasse le linee programmatiche "massime" e gli apriorismi ideologici. Vi è all'interno del movimento anarchico una corrente disponibile alla collaborazione con GL nella prospettiva della lotta antifascista; il rispetto in molti casi diviene vera e propria simpatia, se non accettazione di certi contenuti ideologici e programmatici del giellismo. I rapporti tuttavia resteranno complessi sotto più di un aspetto, non ultimo la palese distinzione ideologica di fondo, che non mancherà mai di essere rilevata. Ciò autorizzerebbe a considerare le vicinanze tra giellisti e anarchici «essenzialmente di ordine politico e legate alle necessità del momento della lotta antifascista, ad un'analisi molto simile sul modo di condurla ${ }^{502}$.

Il patto d'azione siglato tra PSI e Partito Comunista d'Italia (PCd'I) nell'agosto del 1934 sarà inoltre decisivo nel delineare un nuovo campo d'azione politico: nell'aprile del 1935 si separa dal PRI il movimento «Azione Repubblicana Socialista» (ARS) guidato da Fernando Schiavetti ${ }^{503}$ che, insieme a GL, sembra costituire un'interessante sponda politica per il programma di intese ricercato da Berneri. Egli inizierà così ad avvicinarsi sempre più apertamente al movimento giellista, tanto che - come testimonia il suo stesso epistolario ${ }^{504}$ - verrà sovente invitato a partecipare agli incontri presso i circoli GL di Parigi, tanto da farlo

\footnotetext{
${ }^{502}$ G. Manfredonia, Gli anarchici italiani in Francia cit., p. 109, si vedano le pp. 105-109 per una disamina globale sui rapporti tra GL e anarchici. Cfr. inoltre S. Fedele, Il retaggio dell'esilio cit., pp. 95-108, e F. Giulietti, Il movimento anarchico italiano nella lotta contro il fascismo cit., pp. 128-138.

${ }^{503}$ Sulla nascita e le caratteristiche dell'ARS si veda il saggio di E. Signori, L'«Azione Repubblicana e Socialista», in E. Signori, M. Tesoro, Il verde e il rosso cit., in particolare le pp. 84-152.

${ }^{504} \mathrm{Si}$ vedano le lettere di Carlo Rosselli a Camillo Berneri riprodotte in C. Berneri, Epistolario inedito, vol. I cit., pp. 121-122; le risposte di Berneri confermano che egli accettò gli inviti rivoltigli dal vecchio amico.
} 
includere nell'elenco degli affiliati al gruppo dei «Simpatizzanti di Giustizia e Libertà ${ }^{505}$ di cui prende nota la Polizia Politica ${ }^{506}$.

\section{Un progetto per un'Italia federalista e libertaria: indirizzi generali}

Berneri in quello stesso anno è anche il membro più autorevole del Comitato organizzatore del Convegno d'intesa degli anarchici italiani, tenutosi a Sartrouville (Parigi) nell'ottobre del $1935^{507}$. Egli, insieme ai suoi compagni del Comitato, decide d'impegnarsi a stilare un importante rapporto sui compiti di ricostruzione postinsurrezionale degli anarchici, tentando di rompere con forza ma anche con senso pratico l'isolamento di questi ultimi. Il documento redatto, la Relazione $C$. Rapporto sui compiti ricostruttivi degli anarchici nel periodo post-insurrezionale pubblicata per la prima volta da Aurelio Chessa solo nel 1980 - propone importanti coordinate d'azione politica rivoluzionaria:

Un piano d'azione degli anarchici dovrà per conseguenza basarsi su di un maggior decentramento territoriale possibile, su di una suddivisione dei pubblici uffici in rapporto al loro carattere e sfera d'azione, ed affidare all'iniziativa dei gruppi costituiti liberamente tutte quelle funzioni che oggi sono considerate attribuzioni dello Stato.

Bisognerà conseguire un piano di conquista dei comuni, che rappresentano l'espressione minima di governo amministrativo e nell'ambito comunale sviluppare al massimo il concetto della libertà individuale, la messa in comune di tutte le ricchezze e l'autonomia assoluta dagli organi di governo centrale ${ }^{508}$.

Viene particolarmente messo in rilievo, come Berneri aveva sempre sostenuto, il ruolo-chiave che avranno i Comuni nella nuova organizzazione:

I comuni liberi potranno impedire la formazione di un governo centrale [...] a condizione che ogni comune - da sé o in collaborazione libera e volontaria coi vicini - si organizzi razionalmente per mantenere e difendere le conquiste rivoluzionarie, soddisfare i bisogni della popolazione e assicurare i servizi locali.

${ }^{505} \mathrm{Il}$ movimento giellista aveva lanciato questa iniziativa nel giugno dell'anno precedente, vedi Gruppi simpatizzanti di Giustizia e Libertà (SI.G.LA.), «Giustizia e Libertà», I, 15 giugno 1934.

${ }^{506}$ Berneri è segnalato col $n^{\circ} 75$ in Elenchi di affiliati, in ACS, Min. Int., Dir. Gen. PS, Div. Pol. Polit., fascicoli per materia, b. 127, fsc. 1c, Giustizia e Libertà, Parigi, sottofsc. A.

${ }^{507}$ Vedi l'appunto della Div. Pol. Polit., 16 novembre 1935 per la Dir. Gen. PS, AA. GG. RR., Sez. Prima, in ACS, Min. In., CPC, Berneri Camillo, b. 537, fasc. III. Una riproduzione dell'appunto è stata pubblicata in A. Chessa (a cura di), Convegno d'intesa degli anarchici italiani emigrati in Europa (Francia - Belgio - Svizzera), Parigi/Ottobre 1935, Edizioni Archivio Famiglia Berneri, Pistoia,1980, pp. 45-46.

${ }^{508}$ Relazione C. Rapporto sui compiti ricostruttivi degli anarchici nel periodo postinsurrezionale, in A. Chessa (a cura di), Convegno d'intesa degli anarchici italiani cit., p. 27. 
Terra, case, officine, miniere, mezzi di trasporto, depositi di materie prime e manufatti, tutto dovrà diventare "proprietà del comune" (non confondere con nazionalizzazione).

[...] La terra, proprietà comunale inalienabile, sarà concessa agli operai agricoli, braccianti, contadini, mezzadri, fittavoli di oggi - che la lavoreranno in comune nel modo che meglio converrà loro, cioè in cooperative, o sotto l'egida dei sindacati agricoli, o in gruppi di famiglie a seconda dei casi e delle località ${ }^{509}$.

Il Comitato delinea anche il funzionamento della nuova organizzazione comunalista e libertaria, individuando anche la possibilità di riunioni a livello più alto e anche una serie di settori di interesse nazionale:

Nell'orbita limitata del comune, i cittadini si conoscono e quindi possono con cognizione di causa scegliere i loro rappresentanti o delegati, controllarli da vicino e cambiarli ogni qualvolta questi cessino di riscuotere la loro fiducia. [...] Il comune di domani, il Comune Libero, dipenderà in tutto e per tutto dai suoi soli abitanti, nessuna ingerenza di autorità estranee, nessun legame se non quelli volontariamente consentiti e accettati con gli altri comuni affini e limitrofi.

Di tanto in tanto i rappresentati dei comuni si riuniranno in assemblee regionali per consultarsi e risolvere insieme dei problemi d'indole generale, e per certi servizi come le ferrovie, le poste, la navigazione marittima e aerea, l'istruzione, il commercio e i rapporti internazionali, ecc. ecc., si avranno delle assemblee nazionali in cui i delegati dei comuni collaboreranno e delibereranno con i delegati delle corporazioni interessate ${ }^{510}$.

La Relazione $C$ si occupa anche delle future questioni economiche e vale la pena soffermarsi brevemente anche su di esse. Nonostante l'indirizzo prevalente dell'abolizione della proprietà privata, si precisa ad esempio che tale abolizione sarà

limitata naturalmente a quelle sole proprietà e ricchezze che permettono lo sfruttamento dell'uomo - il che vuol dire che certe forme di piccola proprietà saranno tollerate in quantochè non nuocciono al benessere della collettività ${ }^{511}$.

Più avanti il Comitato entra maggiormente nello specifico di come dovrà procedere la nuova organizzazione rivoluzionaria; ritroviamo qui il ruolo-chiave ricoperto dai sindacati e dai soviet (o consigli):

Noi stessi procederemo all'espropriazione di tutti i beni e di tutte le fonti di ricchezza indispensabili alla vita collettiva, man mano che diverremo, insurrezionalmente, padroni del territorio - anche qualora i nostri trionfi non siano che locali. Si espropria dove si è, senza aspettare che la totalità o i tre quarti del paese decida quel che si deve fare. Dobbiamo quindi, in maniera semplice e chiara, preconizzare che:

\footnotetext{
${ }^{509}$ Ibid.

${ }^{510}$ Relazione C. Rapporto sui compiti ricostruttivi degli anarchici cit., p. 28.

${ }^{511}$ Ibid.
} 
a) La proprietà individuale capitalistica deve scomparire;

b) Tutti gli elementi della produzione capitalistica devono divenire proprietà comune della società ed essere amministrati e distribuiti dai produttori stessi;

c) La vita economica del paese dovrà essere affidata ai sindacati e ai comuni.

L'officina apparterrà al comune, quindi alla collettività locale, e sarà gestita nell'orbita del suo funzionamento, dal personale che vi lavora ${ }^{512}$.

Il documento rappresenta un'importante testimonianza della progettualità politica che Berneri e compagni tentarono di portare avanti nel movimento anarchico, nonché dell'influenza esercitata dal lodigiano nell'individuazione delle coordinate entro cui sviluppare detta progettualità.

Se questo scritto risulta poco noto agli storici del pensiero politico, lo è ancor meno il fatto che il gruppo con cui Berneri lavora si spingerà ben oltre generiche riflessioni, arrivando addirittura ad abbozzare una carta costituzionale concepita come documento fondativo. Tale necessità è dichiarata nella stessa Relazione $C$ :

Sarà rimproverato agli anarchici di non possedere un piano costruttivo, di limitare la loro attività e di impiegare tutta la loro energia alla distruzione degli organi vitali della società. Bisogna sfatare questa leggenda e sbozzare sommariamente le linee generali di una organizzazione sociale economica e politica, attendibile domani in Italia e fare in modo che fin dall'inizio della rivoluzione, gli operai, i proletari insorti, realizzino delle conquiste pratiche e concrete ed abbiano subito qualcosa di materiale, di tangibile, che possa resistere a tutte le insidie della demagogia statale e legalitaria $[\ldots]^{513}$.

In questo stimolo alla ricerca di un piano costruttivo appare ancora una volta evidente l'impronta berneriana: la sua proposta politica permea fortemente tanto la Relazione $C$ quanto la parallela Costituzione della Federazione Italiana Comuni Socialisti (F.I.C.S.), i punti più elevati del concretizzarsi del programma anarchico da sempre sostenuto dal lodigiano. Certamente però l'autorevolezza intellettuale di Berneri non deve essere divenuta, nel momento della stesura dei due documenti, un vero e proprio autoritarismo - stiamo pur sempre parlando di un anarchico - dunque è plausibile ipotizzare che egli abbia accettato alcune correzioni e aggiunte da parte dei compagni del Comitato organizzatore ${ }^{514}$. Ciò nondimeno riteniamo eccessivo il giudizio di Adamo secondo cui «nessuno dei due documenti esprime appieno il suo pensiero, presentandosi piuttosto come esito di una collaborazione ${ }^{515}$ : se è vero che

\footnotetext{
${ }^{512}$ Relazione C. Rapporto sui compiti ricostruttivi degli anarchici cit., pp. 29-30.

${ }^{513}$ Ivi, p. 26. I corsivi sono nostri.

${ }^{514} \mathrm{Si}$ veda su questo punto la nota introduttiva di Pietro Adamo a C. Berneri, Costituzione della Federazione Italiana Comuni Socialisti (F.I.C.S.), in Id., Anarchia e società aperta cit., p. 206, ma cfr. anche S. D'Errico, Anarchismo e politica cit., pp. 570-582, e F. Guidi, Nostra patria è il mondo intero! Camillo Berneri e «Guerra di Classe» a Barcellona (ottobre '36novembre '37), edito a cura dell'autore, Brescia, 2010, p. 10.

${ }^{515}$ Nota introduttiva di Pietro Adamo a C. Berneri, Costituzione cit., p. 206.
} 
non possiamo sostenere l'idea di un Berneri quale unico redattore, è altrettanto vero che, alla luce delle ricerche e della storiografia sin qui prodotta, nessun altro dei compagni del Comitato organizzatore né - più in generale - del movimento anarchico aveva potuto sviluppare una così vasta e ampia elaborazione politica; alla luce della nostra ricostruzione, ci pare chiaro che il lodigiano fosse il solo a essersi lanciato (da tempi non sospetti, peraltro) in una riflessione e in un aggiornamento del patrimonio ideologico dell'anarchismo tali da poter condurre alla messa a punto di un simile indirizzo di programma minimo.

In altre parole Berneri, soprattutto dopo tutta la sua irrequieta e persistente opera di critica e ripensamento della tattica e della strategia politica del proprio movimento, era il solo capace di giungere all'appuntamento del Convegno d'intesa con le idee più chiare e profonde, il solo capace di rappresentarvi un saldo e fermo punto di riferimento per quella parte degli anarchici disposta a seguire una certa impostazione della lotta antifascista e rivoluzionaria. Gli indirizzi della Relazione $C$, che verranno travasati ed elaborati nella parallela Costituzione, ci sembrano mostrare in maniera oltremodo evidente una fortissima - ancorché non assoluta - impronta berneriana e riteniamo pertanto che Berneri potesse vedere nella Costituzione una prima formulazione di quel programma minimo per il quale si era a lungo battuto, nonché una possibile piattaforma politica capace di rompere l'isolamento del movimento anarchico; ciò sembrerebbe essere confermato da un altro passaggio della Relazione $C$ :

diffidiamo nel modo più assoluto dei "bolscevichi" e non lasciamoci isolare, anche a costo di patteggiare, in certi momenti e in date circostanze, con gli altri partiti sovversivi: socialisti, repubblicani e comunisti dissidenti ${ }^{516}$.

\section{Un progetto per un'Italia federalista e libertaria: la Costituzione}

La Costituzione, pubblicata solo nel 2001 e la cui importanza non è stata ancora adeguatamente considerata $^{517}$, può essere effettivamente vista come un ipotetico punto di partenza non solo per il programma del movimento anarchico, ma anche per riunire e confrontare le altre forze di una sinistra che, riprendendo il titolo dello studio di Giovanna Angelini, potremmo definire ispirata da «un altro socialismo» vale a dire «un socialismo "dal basso", democratico e liberale, in contrapposizione all'immagine di un socialismo considerato statalista, autoritario e illiberale» ${ }^{518}$. Questo documento rappresenta un vero e proprio punto di svolta sul piano politico

${ }^{516}$ Relazione C. Rapporto sui compiti ricostruttivi degli anarchici cit., p. 26. Interlocutore principale, secondo D'Errico, sarebbe dovuto essere proprio il movimento giellista, cfr. S. D'Errico, Anarchismo e politica cit., pp. 32-33.

${ }^{517}$ All'indomani della sua pubblicazione (2001), il solo studio di S. D'Errico, Anarchismo e politica cit. vi ha dedicato uno spazio considerevole e se ne è parlato solo per alcune battute nello Spazio dibattito (5 maggio 2007), in G. Berti, G. Sacchetti (a cura di), Un libertario in Europa. Camillo Berneri: fra totalitarismi e democrazia. Atti del convegno di studi storici, Arezzo, 5 maggio 2007, Edizioni Biblioteca Panizzi e Archivio Famiglia Berneri-Aurelio Chessa, Reggio Emilia, 2010, p. 267. 
per l'anarchismo, in quanto mai - in onore alla tradizione antiautoritaria - era stata proposta nella sua storia una carta costituzionale che potesse svolgere il ruolo di eventuale documento fondativo per un nuovo assetto statale, sia pure inteso come una repubblica dei consigli federale, socialista e libertaria. Questo spiega perché la Costituzione, ritenuta troppo audace, non sia stata mai riproposta in seguito né agli anarchici disposti a muoversi secondo gli indirizzi di Sartrouville, né ad altri esponenti di forze politiche prossime a un simile disegno. Rimasta a lungo inedita anche dopo la morte di Berneri, la moglie Giovanna Caleffi, prima, e quindi Aurelio Chessa, allorché passò a gestire l'Archivio Famiglia Berneri, hanno scelto di tenerla nascosta. Solo la recente disponibilità di Fiamma Chessa, figlia di Aurelio e attuale responsabile dell'archivio, ha reso possibile la sua pubblicazione nel 2001.

Il documento si apre con alcune «Disposizioni generali», nove articoli in tutto, che tentano un'originale coniugazione tra principi federalisti classici e libertari:

Art. 1 - L'Italia è una Repubblica federale tendente a realizzare il massimo possibile di libertà e di giustizia. I suoi organismi amministrativi, politici e giuridici emanano dal popolo, che ne controlla il funzionamento. La Repubblica è il complesso degli organi nazionali, regionali e municipali.

Art. 2 - L'ordinamento costituzionale della F.I.C.S. è riformabile mediante assemblee costituenti formulanti progetti di riforma che saranno oggetto di plebisciti nazionali.

Art. 3 - La proprietà dei mezzi di produzione è soppressa de jure, ma è tollerato l'ius utendi senza sfruttamento del lavoro altrui.

Art. 4 - Il Comune è il basilare organo amministrativo della socializzazione.

Art. 5 - Le leggi hanno estensione comunale, regionale e nazionale, a seconda del loro oggetto. Esse emanano dalle assemblee comunali, regionali e nazionali e sono approvate o abrogate mediante plebisciti comunali, regionali e nazionali.

Art. 6 - Ogni religione è tollerata, ma sono soppressi i privilegi ecclesiastici.

Art. 7 - La F.I.C.S. non ha una capitale, bensì varie capitali corrispondenti alle varie sfere della sua attività economica.

Art. 8 - La F.I.C.S. è una nazione neutrale e disarmata.

Art. 9 - La F.I.C.S. accoglie le norme del Diritto Internazionale che possono coerentemente incorporarsi nel suo Diritto ${ }^{519}$.

Il Comune è posto, come si può vedere, alla base dell'edificio politico e sociale: non solo la F.I.C.S. è una federazione di Comuni, ma questi ultimi - come peraltro era già stato stabilito nella Relazione $C$ - sono anche gli amministratori delle proprietà socializzate che divengono, in questo modo, proprietà di tutti i cittadini ivi residenti che le ricevono in usufrutto. A questo proposito, il terzo articolo delle «Disposizioni generali» è particolarmente interessante in quanto sembra

\footnotetext{
${ }^{518} \mathrm{G}$. Angelini, L'altro socialismo. L'eredità democratico-risorgimentale da Bignami a Rosselli, Franco Angeli, Milano, 1999, p. 9.

${ }^{519}$ C. Berneri, "Costituzione della Federazione Italiana Comuni Socialisti - (F.I.C.S.), Titolo preliminare, Disposizioni generali", in AFB, conservato separatamente, c. 1. Le sottolineature sono originali. Costituzione cit. pp. 207-208.
} 
riecheggiare le idee di Proudhon circa la distinzione che egli fece tra la proprietà come diritto di dominio, jus in re, e la proprietà intesa come possesso, jus ad rem: il socialista francese giustificò, nella sua memoria Qu'est-ce que la propriété? (1840), il secondo tipo di proprietà, in quanto diritto d'usufrutto dei mezzi di produzione legittimato dal lavoro ${ }^{520}$. Significativo il fatto che Berneri e compagni abbiano evitato di pensare a una capitale, favorendo in questo modo un maggiore decentramento e lasciando così che fossero i centri maggiori delle varie attività economiche a emergere quali punti di riferimento.

Il carattere autonomista e federalista della F.I.C.S. viene sviluppato ulteriormente negli articoli del «Titolo primo - basi costituzionali», di grande interesse soprattutto per quel che riguarda la separazione di competenze tra organi nazionali ed enti locali autonomi:

Art. 11 - Tutti i municipi sono autonomi nelle materie di loro competenza ed eleggono i loro consigli a suffragio universale, diretto e segreto. L'assemblea comunale è l'assemblea del popolo (arengo).

Art. 12 - Le province sono costruite dai gruppi di municipi federati.

Art. 13 - Le regioni possono organizzarsi in unità autonome di fronte alla F.I.C.S., qualora il loro statuto autonomista sia approvato dai due terzi della popolazione.

Art. 14 - La federazione di regioni autonome di fronte alla F.I.C.S. è ammessa a condizione che tali regioni siano limitrofe.

Art. 15 - Sono di esclusiva competenza degli organi nazionali: la rappresentanza della F.I.C.S. all'estero e ogni categoria di relazioni internazionali; acquisto e perdita della nazionalità, sistema monetario; pesi e misure, statistica nazionale.

Art. 16 - I Comuni sono: rurali, industriali, marittimi.

Art. 17 - I Comuni delle sopraccennate categorie costituiscono dei settori nazionali ai quali corrispondono direzioni nazionali a carattere consultivo-esecutivo per le seguenti competenze: finanza generale della repubblica; ordinamento generale delle comunicazioni (postali, telefoniche, telegrafiche, ecc.); utilizzazioni idrauliche e installazioni elettriche; difesa sanitaria; legislazione commerciale e sulla proprietà intellettuale; ordinamento minerario; principi generali sull'agricoltura, foresticoltura e pastorizia in rapporto all'economia nazionale; ferrovie, strade, gallerie, ponti, canali e porti d'interesse generale; legislazione delle acque; caccia e pesca; illuminazione delle coste; servizi dell'aviazione; statistica $^{521}$.

La F.I.C.S. ha una chiara struttura ascendente, è evidente in questa caratteristica l'influenza della lezione politica cattaneana reinterpretata da Salvemini in questo senso; di indubbia derivazione salveminiana sono anche alcune delle competenze lasciate agli organi nazionali ${ }^{522}$. Dovrebbero restare numerose autonomie agli altri

${ }^{520}$ Vedi P.-J. Proudhon, Qu'est-ce que la propriété? Ou Recherche sur le principe du Droit et $d u$ Gouvernement, J.-F. Brocard, Paris, 1840, tr. it. Laterza, Bari, 1967, pp. 49-51.

${ }^{521}$ C. Berneri, "Costituzione della Federazione Italiana Comuni Socialisti - (F.I.C.S.), Titolo Primo, Basi costituzionali”, cc. 1-2. Le sottolineature sono originali. Costituzione cit., p. 208.

${ }^{522}$ Vedi su questi aspetti C. Lacaita, Salvemini interprete e continuatore di Cattaneo, in M. Degl'Innocenti (a cura di), Gaetano Salvemini e le autonomie locali, Lacaita, Manduria-BariRoma, 2007, pp. 53-76, cfr. però le similari idee di O. Zuccarini, Qualche idea 
enti locali, tuttavia ciò è in buona parte controbilanciato dal consistente numero delle competenze per le quali questi devono interagire con le «direzioni nazionali» consultive ed esecutive. Berneri e compagni redattori sembrano prendere atto del numero crescente di attribuzioni che non possono essere totalmente cedute ai diversi comuni, pena il mutamento dell'autonomismo in spinta centrifuga, in potenziale fattore di frammentazione. Tuttavia la struttura, seppure abbozzata, presenta decisi margini di libertà e tenta di ridurre e ripartire quanto più possibile il peso delle «direzioni nazionali», rendendole più che altro organi utili al coordinamento su diverse materie di interesse, dando loro carattere consultivo accanto a quello esecutivo, legandole insomma fortemente alle basi comunaliste dell'organizzazione federale.

La giustizia e l'ordine pubblico, istituzioni storicamente oggetto di critica da parte dell'anarchismo, sono previste dalla Costituzione, ma sono estremamente interessanti e originali le soluzioni proposte per una loro radicale riforma in senso partecipativo e per il loro essere rese controllabili da parte dei cittadini o delle loro assemblee:

Art. 26 - L'ordinamento giudiziario è il seguente: conciliatori in ogni quartiere urbano e in ogni villaggio, eletti dai cittadini e adibiti a risolvere vertenze famigliari, risse di poco conto, ecc.; probiviri corporativi, eletti dalle rispettive categorie professionali, adibiti a risolvere questioni sorte in seno ai sindacati, o al personale delle cooperative o dei municipi; commissioni paritarie, elette dai sindacati e dalle assemblee comunali, nei casi di contrasti tra le cooperative e l'amministrazione comunale, o tra i consigli di quartiere e la seconda; commissioni criminali costituite da tre commissioni: la prima composta da tre medici, la seconda composta di tre esperti di medicina legale, la terza composta di tre psichiatri. Queste sottocommissioni entrano in attività a seconda dei casi.

Art. 27 - I provvedimenti che le commissioni criminali possono prendere a carico dell'autore di un delitto sono: 1) interdizione professionale, temporanea o a vita; 2) interdizione famigliare; 3) interdizione di soggiorno sul territorio del Comune; 4) internamento in un manicomio o in un istituto di rieducazione.

Art. 28 - Ogni detenuto in manicomio deve essere oggetto di una visita collegiale di controllo annuale effettuata da psichiatri incaricati dalla commissione di appello, alla quale potranno rivolgersi sia il detenuto sia i suoi famigliari sia persone amiche. Art. 29 - L'attività comunale in materia di profilassi e di terapeutica della criminalità è integrata e coordinata da una commissione giuridica nazionale, eletta dal Congresso nazionale di assistenza sociale, dal congresso nazionale dei criminalisti, nonché da un Istituto Nazionale di Rieducazione.

Art. 30 - L'ordine pubblico è assicurato da vigili volontari, sorveglianti a turno, un quartiere od un villaggio per incarico della popolazione; da sorveglianti comunali (stradali, forestali, portuari); da sorveglianti sindacali o cooperativi (eletti incaricati dai sindacati o dalle cooperative); da associazioni di volontari (protezioni minorenni, protezione animali, ecc.).

Art. 31 - La polizia criminale è comunale ed è costituita da un relatore (incaricato di esporre il delitto), da un segretario e da agenti dipendenti dall'ufficio di assistenza

sull'ordinamento dello Stato, «La Critica Politica», IV, 25 luglio 1924. 
sociale in numero proporzionale alla popolazione del Comune e alla frequenza dei delitti. L'assemblea comunale controllerà la condotta della polizia criminale, con diritto a commissione d'inchiesta, a revocazione, ecc. ${ }^{523}$

Il carcere, si sarà notato, non compare neanche una volta negli articoli della Costituzione: si parla solamente di istituti di rieducazione e di manicomi, nonché di commissioni di appello a cui possono rivolgersi gli stessi internati dei manicomi. Manca una soluzione per gli istituti di rieducazione, così come non è chiaro quali saranno le sanzioni in cui potranno incorrere coloro che saranno arrestati dalla polizia criminale alle dipendenze del Comune. Quel che appare chiaro è che né Berneri né i compagni si fanno illusioni su un rapido miglioramento degli uomini e delle loro azioni anche se all'interno di un nuovo e più equo sistema, ma sono tuttavia restii a parlare e a entrare nel dettaglio di pene e di detenzione. Resta comunque interessante il fatto che degli anarchici abbiano pensato alla necessità di figure specifiche atte a mantenere l'ordine pubblico, e va inoltre sottolineato che per quest'ultimo non è prevista alcuna direzione nazionale (art. 17) e che resta pertanto una competenza dei singoli Comuni e delle rispettive amministrazioni e assemblee: la F.I.C.S. non dispone insomma di un corpo di polizia nazionale.

Proseguendo, troviamo un elenco dettagliato di diritti civili e politici, su cui degli anarchici hanno certo minori remore a esprimersi:

Art. 37 - Ogni persona ha diritto di manifestare liberamente le proprie idee valendosi di qualsiasi mezzo di diffusione che non costituisca un privilegio sociale ed una speculazione capitalista.

Art. 38 - Ogni italiano può dirigere petizioni, individualmente o collettivamente, agli organi nazionali e può intervenire e prendere la parola nelle assemblee comunali.

Art. 39 - I cittadini maggiorenni di ambo i sessi godranno i medesimi diritti elettorali purché appartenenti ad una categoria di lavoratori.

Art. 40 - È riconosciuto il diritto di riunirsi all'aperto e quello della propaganda orale, senza limiti di luogo né di tempo che non siano quelli stabiliti dai regolamenti comunali.

Art. 41 - Gli italiani possono associarsi o sindacarsi liberamente ${ }^{524}$.

La Costituzione è invece generica e abbastanza vaga al Capo Secondo del Titolo Terzo per quel che riguarda economia e lavoro:

Art. 58 - Il Comune è proprietario della terra, del sottosuolo, delle acque e degli edifici; le cooperative sono proprietarie dei mezzi meccanici, chimici e dei prodotti della terra.

\footnotetext{
${ }^{523}$ C. Berneri, "Costituzione della Federazione Italiana Comuni Socialisti - (F.I.C.S.), Titolo terzo - Diritti e doveri, Capo Primo - Garanzie individuali e politiche", cc. 3-4. Le sottolineature sono originali. Costituzione cit. pp. 209-210.

${ }^{524}$ Ivi, c. 4. Costituzione cit. p. 211.
} 
Art. 59 - La ricchezza artistica e storica del paese appartiene alla Nazione, che ne assicura la custodia e la conservazione mediante l'Ente nazionale dei monumenti.

Art. 60 - Non è tollerata la proprietà privata che a titolo di ius utendi, senza diritto ereditario e senza salari ${ }^{525}$.

Salvo l'articolo 59, si può notare come questa sezione ricalchi in tutto e per tutto i contenuti della Relazione $C$ senza però svilupparli ulteriormente. Per quanto attiene il lavoro, le garanzie costituzionali sono ancora più vaghe:

Art. 61 - Il lavoro è un obbligo sociale.

Art. 62 - È proibito il lavoro dei fanciulli e delle donne gravide.

Art. 63 - La giornata di lavoro ed il compenso sono stabiliti da commissioni sindacali comunali ${ }^{526}$.

Riteniamo che queste due sezioni siano state solo abbozzate per via della delicatezza di questi temi, non solo perché avevano ricoperto spazio minore anche nella Relazione $C$, ma anche e soprattutto in vista di un confronto con altre forze politiche: su questi temi si sarebbe probabilmente dovuto transigere molto più che in altri, dunque Berneri e compagni del Comitato devono aver preferito limitarsi a fissare una serie di capisaldi da sviluppare.

La Costituzione è invece ricca in merito agli organi rappresentativi ed esecutivi, e ciò è molto interessante poiché qui molti dottrinarismi e parole d'ordine tradizionali dell'anarchismo vengono accantonati e sacrificati. Il tentativo di coniugare la delega della rappresentanza - storicamente rifiutata dagli anarchici con un maggior peso delle istanze di partecipazione "dal basso" diventa allora il primo dei problemi:

Art. 64 - La potestà legislativa risiede nel popolo che la esercita per mezzo di plebisciti.

Art. 65 - I Consigli Comunali sono eletti per suffragio universale, eguale, diretto e segreto; i Consigli provinciali sono eletti dai Consigli comunali; i Consigli regionali sono eletti dai Consigli provinciali; i Consigli nazionali sono eletti dai consigli regionali.

Art. 66 - La durata del mandato è fissata da un plebiscito.

Art. 67 - Ogni delegato è destituibile su richiesta del $50 \%$ degli elettori ${ }^{527}$.

Val la pena sottolineare, ancora una volta, come il Comune sia il vero elementochiave dell'intera organizzazione federalista e libertaria della F.I.C.S.: è a partire da

${ }^{525}$ C. Berneri, "Costituzione della Federazione Italiana Comuni Socialisti - (F.I.C.S.), Titolo terzo - Diritti e doveri, Capo Secondo - Economia", c. 6. La sottolineatura è originale. Costituzione cit., p. 212.

${ }^{526}$ Ibid.

${ }^{527}$ C. Berneri, "Costituzione della Federazione Italiana Comuni Socialisti - (F.I.C.S.), Titolo terzo - Diritti e doveri, Capo Secondo - Organi esecutivi e rappresentativi”, c. 6. Costituzione cit., pp. 212-213. 
esso che si risale progressivamente fino agli organi nazionali, in un concatenamento che - servendoci di una metafora - rende il vertice del triangolo molto più vicino alla base; vertice che peraltro ha soprattutto, come abbiamo visto, attribuzioni di coordinamento, non di governo vero e proprio. Proseguendo, possiamo notare come siano stati immaginati e previsti dei forti contrappesi agli organi nazionali, anche per quel che concerne le questioni internazionali e la rappresentanza della F.I.C.S. all'estero:

Art. 70 - Le convenzioni internazionali sono di spettanza dei consigli nazionali, ma vanno ratificate dai consigli regionali. I trattati internazionali di carattere politico vanno ratificati da un plebiscito nazionale.

Art. 71 - Il popolo esercita il diritto di iniziativa mediante proposte ed esercita il controllo mediante voti di censura e sfiducia. La procedura e le garanzie dei referendum e della iniziativa popolare sono fissate dalla Costituente permanente: assemblea annuale di tutti i consigli regionali, incaricati dai mandanti di quelli provinciali e comunali.

Art. 72 - Il rappresentante della F.I.C.S. è la personificazione simbolica della Nazione. Esso è nominato annualmente mediante plebiscito. La funzione è soltanto rappresentativa.

Art. 73 - I Commissari nazionali sono nominati dai Consigli nazionali e costituiscono le direzioni generali dei vari settori.

Art. 74 - Gli ambasciatori sono nominati dai Consigli nazionali, i consoli sono nominati dagli italiani all'estero ${ }^{528}$.

Da rilevare la presenza di un organismo del tutto particolare, una «Costituente permanente». La Costituzione non è affatto un punto fermo, non arresta e cristallizza il processo rivoluzionario, ma anzi lo tiene in vita attraverso questo organo che ha il compito di stabilire modalità e regole attraverso cui il popolo può partecipare e controllare gli organi amministrativi, politici e giuridici della F.I.C.S., come previsto dall'art. 1: non esiste, come si è potuto vedere, una sola decisione che possa essere presa, una sola regola che possa essere imposta, una sola carica che possa essere ricoperta senza l'approvazione o comunque sia contro il parere dei rappresentati.

La Costituzione rappresenta un documento centrale e, va ribadito, senza precedenti né esempi analoghi successivi in ambito anarchico. In particolare è interessante osservare il tentativo costante di salvaguardare e di affermare, all'interno di quello che in fin dei conti è un atto normativo, principi cardinali libertari e federalisti: è un segnale forte di ricerca di concretezza operativa e di dialogo politico senza che questo dovesse comportare l'abbandono dei propri punti di riferimento ideologici. Ciò conferma e ben rappresenta la maturazione storica della tendenza berneriana volta ad aggiornare la battaglia anarchica in senso propositivo, rispetto alla quale la Costituzione mostra una sorprendente continuità. Si è trattato insomma di un'occasione importante per fissare i punti di quel "programma minimo" che Berneri auspicava da tanto tempo, tanto più in un

\footnotetext{
${ }^{528}$ Ivi, cc. 6-7. Costituzione cit., pp. 212-213.
} 
momento in cui i rapporti col giellismo si facevano sempre più stretti e la possibilità di un'azione comune iniziava a profilarsi.

Alla luce di quanto fin qui osservato - e soprattutto tenendo presenti i passi della Relazione $C$ che abbiamo citato e che ritornano nell'abbozzato testo costituzionale non riteniamo affatto convincente la ricostruzione di De Maria: lo studioso sostiene che il progetto costituente non «risulta del tutto chiaro» e lo considera, in modo a nostro avviso quanto meno riduttivo, nulla più che «uno dei frammenti della riflessione politica di Berneri, da affiancare ad altri, senz'altro più significativi» ${ }^{529}$. La Costituzione ci sembra rappresentare invece senz'altro qualcosa di più di un frammento significativo, appare anzi il concretizzarsi nel modo più compiuto di quel «anarchismo attualista», di quel concretismo rivoluzionario di Berneri, e doveva certo rappresentare anche una prima bozza per organizzare una piattaforma per un'alleanza con GL o con altre forze disponibili a lavorare su un programma federale, autonomista, rivoluzionario e tendenzialmente libertario: un documento centrale per comprendere quanto fosse viva, almeno in una parte del movimento, la volontà di rompere il proprio isolamento per raggiungere una base di intesa con interlocutori politici affini. Altresì non condividiamo il giudizio di Carrozza, secondo il quale l'attività di Berneri «non si dà mai come un insieme organico di risposte in positivo, ma sempre e comunque come tentativo di stimolare la riflessione, di mettere in luce le contraddizioni, di problematizzare $[\ldots] \gg{ }^{530}$. $\mathrm{Ci}$ sembra quasi superfluo sottolineare, ancora una volta, come la Costituzione sia stato un primo, per quanto abbozzato, tentativo di costruire un programma pratico e attuabile per il movimento anarchico, un primo e importante passo verso un insieme organico di risposte in positivo. È vero che molti dottrinarismi e parole d'ordine vengono accantonati in questo documento, $\mathrm{ma}$ in realtà è nostra opinione che già il solo fatto di aver voluto tratteggiare un progetto costituente fosse una rottura profonda con alcuni principi dell'anarchismo tradizionale.

Il programma della rivoluzione e della ricostruzione su cui dialogare con altre forze politiche antifasciste tuttavia non doveva essere tale da violare completamente i principi dell'anarchismo; dunque, pur rinunciando ad alcune pregiudiziali, è interessante notare come questa ricerca e questo lavoro di stesura siano avvenute all'insegna di soluzioni e di idee in grado di mantenere insieme proposte pragmatiche e salvaguardia di importanti principi cardinali libertari.

\section{Sviluppi della riflessione e confronto con il «socialismo federalista liberale»}

La Costituzione forse non riflette integralmente le idee di Berneri, ma alla luce della nostra ricostruzione riteniamo che vi sia fortemente e inequivocabilmente

\footnotetext{
${ }^{529}$ C. De Maria, Camillo Berneri cit., p. 164 (nota a piè di pagina). Vedi anche Ivi, pp. 96-97.

${ }^{530} \mathrm{G}$. Carrozza, Alcuni elementi per la comprensione dei rapporti tra Berneri ed il movimento anarchico, in Atti del Convegno di studi su Camillo Berneri, Milano, 9 ottobre 1977, La Cooperativa Tipolitografica, Carrara, 1979, p. 32.
} 
percepibile l'influenza del pensiero politico da lui sviluppato. La sua ricerca di un "programma minimo" per la lotta antifascista e la rivoluzione sociale e federalista libertaria sono state del tutto particolari e originali all'interno del movimento anarchico, e trovano finalmente in questo documento un importante punto fermo. $\grave{E}$ tuttavia difficile pensare che egli si ritenesse soddisfatto, di certo queste non potevano che essere le linee generali, il nucleo di un lavoro che avrebbe dovuto con ogni probabilità diventare più ampio e articolato; in altre parole, la Costituzione è parte essenziale di quell'agognato programma, ma di certo non si può dire che questo coincida con quella in tutto e per tutto. Avrebbe potuto però senza dubbio diventarlo nel corso del tempo e avrebbe forse potuto ricoprire un ruolo di importanza inestimabile per il movimento anarchico, o almeno per una parte di esso: non solo Berneri e compagni si sarebbero potuti presentare a dei potenziali alleati politici con un documento chiaro e ben sviluppato, recante le coordinate generali della nuova organizzazione federalista e libertaria nazionale, ma $-\mathrm{e}$ forse è ancor più significativo - finalmente il movimento avrebbe potuto avvalersi di un piano d'azione con importanti indirizzi e coordinate per la rivoluzione libertaria.

Gli anarchici avrebbero potuto finalmente costituire un'alternativa influente e concreta, capace di calamitare tutti i movimenti e i partiti contrari tanto all'asse tra socialisti e comunisti quanto alle ipotesi moderate di ristabilimento dell'antico ordine liberal-democratico; tutto sarebbe dipeso dalla capacità del loro programma di offrire risposte il più possibile pratiche e capillari. Il movimento sarebbe così potuto diventare un punto di riferimento, senza isolarsi nella propria purezza e senza ridursi a giocare un ruolo di secondo piano: più gli anarchici sarebbero stati disponibili a uscire dal generico e piano campo dei principi per incamminarsi sul complesso e accidentato terreno della politica, più sarebbero riusciti a imprimere un carattere libertario alla rivoluzione sociale e antifascista.

\section{Un laboratorio politico anarchico}

Un documento a cui non è mai stata data sufficiente considerazione fino ad oggi nelle ricostruzioni sulla storia del movimento anarchico in esilio o sulla vicenda di Berneri è altamente eloquente in questo senso: è una Circolare priva di luogo e datazione, ma che riteniamo certamente successiva al Convegno di Sartrouville (ottobre 1935) poiché inviata dal Comitato organizzatore e volta a stimolare la partecipazione dei compagni nell'elaborazione di un «programma insurrezionale e di realizzazione immediata per il nostro movimento»:

il Convegno ha tracciato le linee generali della nostra condotta e della nostra azione rivoluzionaria. 
Restano a esaminare le soluzioni pratiche - e per così dire: particolari - dei diversi e complessi problemi della vita sociale che intendiamo riorganizzare domani su basi nuove ${ }^{531}$.

Il Comitato invita chi desidera collaborare a scegliere le questioni in cui si ritiene più competente, e specifica:

In apposite riunioni, discuteremo i problemi uno per uno e tireremo delle conclusioni. I compagni che non vivono nella regione parigina - e che per conseguenza non potranno partecipare alle riunioni, come quelli che - pur essendo vicini - ne saranno impediti per una ragione qualsiasi, sono pregati di mandare delle relazioni scritte.

In tal modo intendiamo continuare e completare la missione del Convegno d'Ottobre.

È ben esplicito che: le soluzioni proposte o suggerite per ogni problema specifico devono rapportarsi al periodo insurrezionale.

Ogni relatore deve tener presente che si tratta di soluzioni e realizzazioni pratiche, immediate e cioè applicabili nelle prime fasi della rivoluzione, e non perdersi nei miraggi dell'avvenire lontano, di cui tratteremo più tardi, quando la rivoluzione sarà in atto e le prime tappe realizzate ${ }^{532}$.

È facile riconoscere nelle frasi di questa Circolare $\mathrm{i}$ motivi principali dell'attivismo e dell'atteggiamento intellettuale berneriano: se anche non fossero state stese dal lodigiano in persona, di certo risentono fortemente della sua influenza. Dunque, una parte del movimento anarchico era pronta a mettersi in gioco e a dar corso allo sviluppo di un programma rivoluzionario, studiando i «problemi della vita sociale» e ponendo le basi di un laboratorio politico quale il Berneri aveva sempre auspicato. Alla circolare era allegato un elenco di problemi da esaminare, e vale la pena scorrerlo tutto per rendersi conto di quale ampiezza di respiro dovesse avere il lavoro di elaborazione programmatica che Berneri e il Comitato organizzatore intendevano intraprendere:

Problema del Lavoro

La rimunerazione del Lavoro

Problema dell'Abitazione

I Trasporti Pubblici

Poste Telefoni e Telegrafi

Teatro e Divertimenti

Il Servizio Sanitario

Il Commercio di Dettaglio e i Rifornimenti

L'Istruzione Pubblica

${ }^{531}$ Circolare s. 1., s. d. (ma Parigi, novembre-dicembre 1935), in ACS, Min. Int., Dir. Gen. PS, Div. Pol. Polit., fascicoli per materia, b. 25, fsc. 1, Francia, anarchici italiani. Il documento è riprodotto in A. Chessa (a cura di), Convegno d'intesa degli anarchici italiani cit., p. 5.

${ }^{532} \mathrm{Circolare}$ cit. Il corsivo è nostro. 


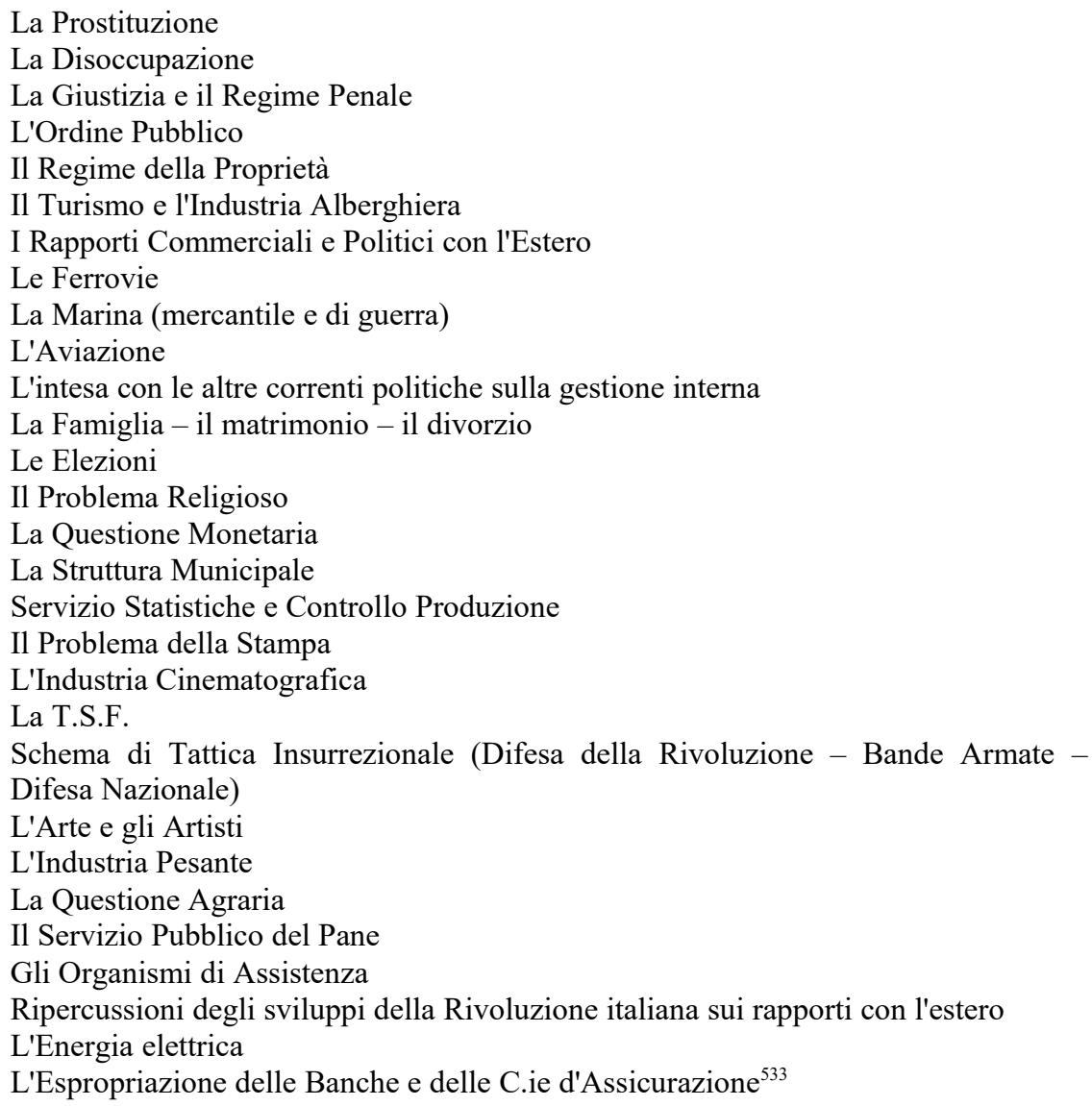

La preparazione del movimento anarchico avrebbe dovuto essere, come si può vedere, vasta, capace di offrire soluzioni libertarie e percorribili per ciascuno dei problemi proposti; a lavoro concluso, la Costituzione sarebbe divenuta con ogni probabilità un documento più ricco, completo e articolato, capace di dare al movimento un programma pratico e di metterlo così in una posizione di primo piano tra le forze antifasciste di ispirazione federalista e autonomista. Aspetto importante era però, appunto, anche quello del dialogo con partiti e movimento prossimi agli anarchici, giacché né Berneri né nessun altro compagno erano certo convinti di poter avanzare da soli e, al tempo stesso, non avrebbero mai voluto o potuto accettare di imporre ad altri la propria leadership. Conseguentemente, la necessità di un programma approfondito e il più possibile completo diventava ancora più urgente poiché, presumibilmente, maggiore sarebbe stato il lavoro di studio e di elaborazione in questo senso, minori sarebbero stati i sacrifici da fare alle proprie istanze. Gli

${ }^{533}$ Ibid. L'elenco è riprodotto in A. Chessa (a cura di), Convegno d'intesa degli anarchici italiani cit., p. 3. 
esiti, insomma, sarebbero stati tanto più libertari quanto più forte e incisiva sarebbe stata la preparazione degli anarchici.

La Costituzione della F.I.C.S. - pur nella sua provvisorietà - ci sembra confermare questa ipotesi: è un documento "aperto" e, come abbiamo visto, caratterizzato dall'accettazione di numerosi compromessi rispetto alle idee anarchiche; attagliato alle condizioni storiche e sociali dell'Italia e passibile di ulteriori sviluppi e messe a punto, avrebbe potuto offrire una piattaforma programmatica organica da discutere con altre forze di ispirazione socialista, autonomista e federalista.

\title{
Appunti berneriani complementari: il nazional-anarchismo
}

Il legame tra la Costituzione della F.I.C.S. e il percorso intellettuale di Berneri che, come abbiamo fin qui mostrato, è profondamente segnato dal dibattito politico e culturale svoltosi in Italia intorno a questi temi, oltreché dalla necessità per il movimento anarchico di darsi un programma strettamente connesso coi problemi della vita nazionale - è confermato da una serie di appunti discontinui di questo periodo, significativamente raccolti sotto il titolo Il nazional-anarchismo ${ }^{534}$. Ne estrapoliamo un passaggio che contribuisce a comprendere come Berneri stesso qualificasse il proprio pensiero come un innesto di influenze varie all'interno di una visione politica sostanzialmente anarchica:

\begin{abstract}
Il mio naz[ional] an[archismo] Cattaneo, letto, citato e consigl[iato] con scandalo dei caporalucci dell'ortod[ossia] Kropot[kiniana]; simpatia per il protestant[esimo]; probl[emismo] di Salv[emini]; critica liberista allo St[ato]; Gobetti - si richiama a Pareto, a Einaudi, ecc. ben più che ai liberali inglesi; Critic[a] Pol[itica] - nazionalrepubbl[icana]; revisionismo lib[erale]; ribel[lione] al dom[inio] di Londra, la Mosca della I Int[ernazionale]; la lotta tra Maz[zini] e Bak[unin], così ben illustr[ata] da Nello Rosselli e da Max Nettlau, fu in gran parte lotta tra l'astratt[ismo] ideol[ogico] di Mazzini e il concretismo soc[ialista] - Piscane - lett[era] di Bak[unin] sulla riv[oluzione] pol[itica] al Bertani ${ }^{535}$
\end{abstract}

Ma se questo estratto degli appunti del 1935 non fa che confermare ulteriormente quanto abbiamo già osservato nel corso di questo lavoro, è un altro di questi piccoli fogli che attira la nostra attenzione. Con ogni probabilità coevo alla stesura della Costituzione, giacché vi fa esplicito riferimento, esso testimonia chiaramente quanto abbiamo illustrato nel corso di questa trattazione e ci permette di aggiungere qualcosa di nuovo a quanto è stato fin qui sostenuto circa questo insieme

\footnotetext{
${ }^{534}$ C. Berneri, "Il nazional-anarchismo", in AFB, fondo C. Berneri, cass. IV, Opere di carattere politico, $\mathrm{n}^{\circ} 1$, Anarchia e anarchismo. Si tratta di una serie di appunti che, seppur citata, non è ancora mai stata riprodotta integralmente; questi sono stati stesi nel corso del 1935, come dimostrerebbe, tra l'altro, il fatto che alcuni siano stati presi su buste di lettere indirizzate a Berneri nel gennaio di quello stesso anno, vedi C. De Maria, Camillo Berneri cit., p. 142 (nota a piè di pagina).

${ }^{535}$ Ibid.
} 
di appunti dalle ricostruzioni sul pensiero politico dell'anarchico lodigiano: vi è stato visto soprattutto un Berneri che tratteggia la propria formazione e le sue influenze culturali $^{536}$, ma noi riteniamo invece che in questi appunti vi sia soprattutto un Berneri che intende dare ulteriore sviluppo a quelle linee-guida che hanno favorito il maturare del suo anarchismo sui generis, travasandole nell'ambizioso progetto politico per cui si è a lungo impegnato e sbozzando alcune idee circa ulteriori articolazioni del programma e il possibile funzionamento di alcuni organi della F.I.C.S.

Le soluzioni sono alquanto originali, ad esempio, per quanto riguarda le sedi delle «direzioni» a carattere consultivo ed esecutivo dei settori di interesse nazionale dell'articolo 17 della Costituzione:

Dire[zione] gen[erale] Ferrovie - treno speciale | Trasporti marittimi: nave | Istruz[ione] pubbl[ica] Firenze Industrie: Torino | Commercio: Milano | Belle arti Venezia | agricoltura: carovana auto | Comunicazioni: carovana auto | Aviazione: dirigibile | Colonie: nave | Igiene: nave e carovana auto | Esteri: in viaggio ${ }^{537}$

Berneri stava inoltre iniziando a occuparsi di uno specifico problema, quello degli italiani residenti in Francia, sommariamente affrontato nell'articolo 74 della Costituzione. Egli ipotizza anche in questo caso una soluzione di tipo consiliare con specifiche autonomie e una serie di meccanismi attraverso cui favorire la massima partecipazione dei residenti all'estero sulla scelta e il controllo dei propri rappresentati:

Colonie: tutti gli italiani dim[oranti] a Parigi e banlieue cost[ituiscono] la colonia ital[iana] di Parigi - che ha una casa degli italiani (sale di lettura, albergo, teatro, ecc), una scuola italiana, un ospedale italiano (o una clinica) e un consolato - Il console è eletto mediante plebiscito promosso dal Consiglio degli emigr[ati] Ital[iani] di Parigi e dintorni - e rimane in carica fino a quando non vi è il $10 \%$ degli it[aliani] della Colonia che ne richiede la destituzione. Il Console è coadiuvato e controllato da 2 vice-consoli, anch'essi nominati dal plebiscito. I 3 nominano i funz[ionari] seguendo le norme dello Statuto della Colonia italiana di Parigi e dintorni - Le ambasciate dipendono dal Consiglio degli emigrati e dalla direz[ione] gener[ale] delle colonie e da quella dei rapporti con l'estero. Gli ambasciatori vengono eletti da un'assemblea di consoli e di vice-consoli. La nomina deve essere ratificata (o può essere respinta) dalla direz[ione] gen[erale] affari esteri e da quella delle colonie - Nel $2^{\circ}$ caso è promosso un plebiscito dei consigli delle colonie it[aliane] della Francia. In caso che l'accordo non sia raggiunto si promuove un plebiscito nelle colonie della Francia - L'Ambasc[iata] ital[iana] è a Marsiglia e non a Parigi ${ }^{538}$.

\footnotetext{
${ }^{536}$ Vedi P. Adamo, Introduzione a C. Berneri, Anarchia e società aperta cit., pp. 77-79, e C. De Maria, Camillo Berneri cit., pp. 141-145.

${ }^{537} \mathrm{C}$. Berneri, "Il nazional-anarchismo" cit.

${ }^{538}$ Ibid. Le sottolineature sono originali.
} 
Infine, Berneri riunisce sotto l'evocativo nome «Campi di Maggio» tutto l'insieme degli organi consiliari politico-territoriali ed economico-sociali della F.I.C.S. che vanno a sostituirsi, di fatto, al governo:

I Campi di Maggio - I Congressi corporativi permanenti - I Consigli regionaliprovinciali-comunali-corporativi locali-le adunate cons[igli] di fabbrica, di scuola, ecc.

Federaz[ione] Comun[i] Soc[ialisti] It[aliani] (F.C.S.I.) - Bandiera rossa con F.C.S.I.

- Non vi è capitale - governo è il Campo di Maggio - Capitale: ovunque il lavoro $\operatorname{arde}^{539}$

Già l'articolo 7 aveva stabilito che la F.I.C.S. non avesse una capitale, ma quello che sorprende è l'utilizzo del termine «governo» da parte di un anarchico, che può essere però spiegato come l'ennesima interpretazione originale che il lodigiano ha dato a un termine lontano dall tradizione politica anarchica, quella sintesi direttiva di comunità autonome e federate evocata nella sua concezione anarchica dello Stato. In altre parole, la F.I.C.S. rappresenta una sorta di "Repubblica dei consigli federalista e libertaria" e l'insieme di tali consigli "governa" nel senso che si occupa dell'amministrazione delle cose, subentrata infine al governo sugli uomini. Berneri elabora certamente le sue idee in questi ultimi mesi del 1935 in cui pare finalmente possibile che parte degli anarchici sia pronta a confrontarsi su questioni concrete, come dimostra un'altra Circolare senza luogo e senza data giunta alla Divisione Polizia Politica ${ }^{540}$ : essa invita a una riunione per sabato 21 dicembre 1935 alle ore 14 per discutere sul problema del lavoro che, come si ricorderà, era proprio una delle sezioni meno sviluppate della Costituzione.

Berneri e compagni del Comitato stanno incominciando a mobilitare le forze di almeno una parte del movimento anarchico e molto probabilmente il risultato di questi incontri avrebbe favorito un approfondimento dell'ancora schematica Costituzione. Restava da vedere quali avrebbero potuto essere i loro interlocutori politici.

\section{«Avversari un poco cugini»}

Pur senza parlare di costituenti e di piattaforme comuni, già l'anarchico Umberto Consiglio era tornato a esprimere la preoccupazione, con una lettera pubblicata su «Giustizia e Libertà» del 22 novembre 1935, che le pur accettabili simpatie e collaborazioni tra $\mathrm{i}$ due movimenti potessero sfociasse in un possibile assorbimento, invitando $i$ suoi compagni a non perdere di vista la funzione specifica dell'anarchismo:

${ }^{539} \mathrm{C}$. Berneri, "Il nazional-anarchismo" cit. Le sottolineature sono originali.

${ }^{540}$ Circolare s. 1., s. d. (ma Parigi, dicembre 1935), in ACS, Min. Int., Dir. Gen. PS, Div. Pol. Polit., fascicoli per materia, b. 25, fsc. 1 cit. 
Noi ci si deve sforzare di restare noi stessi, affinché si rimanga il nucleo che domani, assieme ai reduci delle galere e dei confini, si possa divenire la pattuglia per la ripresa integrale della nostra azione ${ }^{541}$.

Il timore di Consiglio è che GL, movimento nato sotto il fascismo e dal tormento della gioventù repubblicana e socialista, possa un domani farsi partito e diventare élite radicale dimenticando i suoi principi più audaci. Tuttavia egli afferma che si tratta di una preoccupazione che riguarda l'avvenire e la posizione attuale dei giellisti è in buona parte condivisibile. Inoltre, poiché gli anarchici non possono da soli imprimere un segno negli avvenimenti prossimi, può risultare utile legarsi a quei movimenti o partiti con cui si hanno «maggiori affinità elettive»; l'importante è che i suoi compagni non divengano così «pattuglia ondeggiante d'individui in cerca affannosa del migliore alleato cui accodarsi $[\ldots]\rangle{ }^{542}$.

La postilla alla lettera, da attribuirsi con ogni probabilità a Carlo Rosselli, sottolinea allo stesso modo la consonanza tra ideologia giellista e anarchica:

Al pari degli anarchici, noi siamo recisamente opposti ad ogni forma di dittatura e di oppressione, ad ogni ipertrofia statale e, in genere, ad ogni forma di organizzazione sociale in cui non si faccia largo, e sempre più largo, posto alle autonomie e iniziative dei singoli e dei gruppi; e diamo grande importanza ai problemi di moralità e di cultura ${ }^{543}$.

La divisione sussiste, secondo Rosselli, in merito all'eccessiva ripugnanza dell'anarchismo verso le forme di organizzazione, ma soprattutto al mantenersi da parte di troppi anarchici sulle vecchie formulazioni del pensiero anarchico tradizionale ottocentesco, che rappresentava una visione di un mondo assai lontano da quello attuale. In ragione di ciò egli afferma che bisogna considerare i problemi di una società libera alla luce dei tempi contemporanei, utilizzando una formula che in un certo modo suonerà per gli anarchici come concorrenziale: «Urge cioè diventare libertari del XX secolo, come talvolta noi - e non per civetteria - ci proclamiamo, per tentare finalmente una grande esperienza pratica ${ }^{544}$. Tale rivendicazione del titolo di «libertari del XX secolo» suggerisce che Rosselli considerasse in qualche modo l'anarchismo come superato dal proprio movimento

${ }^{541}$ U. Consiglio, Gli anarchici e "G. e L.", «Giustizia e Libertà», II, 22 novembre 1935. Umberto Consiglio (1889-1964) è stato un anarchico italiano. Divenuto militante dopo la Prima Guerra Mondiale, collabora alla stampa e si fa notare per il suo attivismo antifascista. Emigrato in Francia nel 1926, dialoga con vari movimenti e partiti, tra cui GL. Accorso in Spagna per la Guerra Civile, vi si impegna fino alla sconfitta del fronte repubblicano; tornato in Francia, si arruolerà volontario nell'esercito francese, ma verrà catturato dalla polizia di Vichy e condotto a Dachau, cui riuscirà per miracolo a sopravvivere. Vedi «Consiglio, Umberto», in M. Antonioli et al. (diretto da) Dizionario biografico degli anarchici italiani, vol. I, Edizioni Biblioteca Franco Serantini, Pisa, 2003, pp. 434-436.

${ }^{542}$ Ibid.

${ }^{543}$ [C. Rosselli], Risposta di G.L. a U. Consiglio, Gli anarchici e "G. e L." cit. ${ }^{544}$ Ibid. 
politico, anche perché ne aveva parzialmente ripescato la tradizione nella sua critica del marxismo e nel tentativo di formulare una nuova elaborazione teorica socialista $^{545}$. Berneri, che come abbiamo visto sta già da tempo lavorando per un rinnovamento dell'anarchismo che oltrepassasse i dogmatismi e gli assoluti ideologici, non si sente affatto superato, tanto meno da GL, e non mancherà di far sentire la sua voce in merito. La prossimità alle idee e al percorso intellettuale del vecchio amico Carlo Rosselli ${ }^{546}$ non sono più forti del bisogno che il Berneri sente di dover nettamente differenziare il proprio movimento da quello dei giellisti, con cui pure sta cercando una fertile collaborazione.

Sul finire del 1935 e all'indomani del ricordato Convegno di Sartrouville, Berneri interviene direttamente su «Giustizia e Libertà» proprio a proposito di questo tema, avviando un breve ma intenso scambio con il fondatore di GL. Il dibattito, finora solo parzialmente analizzato in particolare dagli studiosi di Rosselli ${ }^{547}$, ci sembra far emergere un aspetto su cui non si è ancora fatta sufficiente attenzione, e che va al di là delle già studiate prossimità e divergenze ideologiche tra Rosselli e Berneri, o dei rapporti tra i movimenti anarchico e giellista. Esso rivela e conferma con chiarezza - nonostante le critiche portate al movimento e nonostante la tendenza concretista per un «anarchismo attualista» che lo aveva portato a stilare un progetto costituzionale - la piena adesione di Camillo Berneri all'ideale anarchico, da un lato; dall'altro, mostra quanto Rosselli e il suo movimento fossero

${ }^{545} \mathrm{Si}$ veda in particolare C. Rosselli, Socialisme libéral, Librairie Valois, Paris, 1930, tr. it. RCS Quotidiani, Milano, 2011, soprattutto le pp. 122-166, in cui compaiono cenni alle idee di Proudhon e alla «scarna tradizione socialista italiana» in cui sono inclusi i libertari Pisacane e Cafiero. Cfr. anche C. Malandrino, Socialismo e libertà cit., pp. 114-115 e 125129.

${ }^{546} \mathrm{Su}$ prossimità e divergenze tra posizioni e idee politiche di Rosselli e Berneri si veda G. Berti, Berneri e Rosselli: anarchismo e socialismo liberale a confronto, in M. Nacci (a cura di), Figure del liberalsocialismo, Centro Editoriale Toscano, Firenze, 2010, soprattutto alle pp. 75-81; cfr. inoltre E. Acciai, Berneri e Rosselli in Spagna. L'esperienza della "Sezione italiana della colonna Ascaso", in G. Berti, G. Sacchetti (a cura di), Un libertario in Europa cit., pp. 82-95, e S. D'Errico, Anarchismo e politica cit., pp. 352-354.

${ }^{547}$ Il dibattito è stato integralmente riprodotto in C. Berneri, Pietrogrado 1917 Barcellona 1937 cit., pp. 161-176, e in Id., Anarchia e società aperta cit., pp. 199-204 e pp. 152-158. Oltre alle più equilibrate ricostruzioni di S. Fedele, Il retaggio dell'esilio cit., pp. 106-108 e di C. Malandrino, Socialismo e libertà cit., pp. 129-135, si può facilmente notare come l'attenzione sullo scambio tra i due sia dovuta soprattutto agli studi sul Berneri, laddove nelle antologie degli scritti di Rosselli se ne trova in genere solo una minima traccia - cfr. in particolare C. Rosselli, Scritti dell'esilio, vol. II, Dallo scioglimento della Concentrazione antifascista alla Guerra di Spagna (1934-1937), a cura di Costanzo Casucci, Torino, Einaudi, 1992, pp. 261-265, che riporta un intervento del solo Rosselli senza ricostruire l'intero dibattito. In generale, gli stessi studi sul giellismo tendono a lasciare sullo sfondo il confronto col movimento anarchico e con Berneri, se non per imputare a questi ultimi le responsabilità dello scioglimento della «Colonna Ascaso» in Spagna. 
invece lontani dall'aver recuperato il patrimonio ideologico libertario con piena, concreta e propositiva convinzione.

Nel suo primo intervento, il professore anarchico rileva che un elemento di contatto forte tra $\mathrm{i}$ due movimenti è la comune radice antiautoritaria, tanto da arrivare ad affermare che

Se, per citare un esempio, Carlo Rosselli avesse avute presenti le critiche al marxismo di Covelli, di Cafiero, di Malatesta, di Tcherkesof, di Merlino, di Gille, di Fabbri e di altri scrittori anarchici, avrebbe constatato che il suo revisionismo di 'Socialisme Libéral' non era che una sintesi della critica anarchica. Leggendo gli scritti degli intellettuali giellisti, incontro continuamente vecchie conoscenze: da Godwin a Malatesta. Ma come Sorel profittò in Italia della poca fortuna di Proudhon, così i libertari del XX secolo profittano della cattiva conoscenza che $\mathrm{i}$ più, anche tra le persone colte, hanno del pensiero anarchico. Non voglio dire che vi sia dolo. Vi è capillarità ${ }^{548}$.

Berneri contesta a Rosselli di voler annettere, a sé e al suo movimento, l'eredità del socialismo libertario, approfittando del fatto che si ha scarsa e cattiva conoscenza dell'anarchismo. A maggior ragione dopo gli sforzi teorici intorno al progetto costituzionale che abbiamo precedentemente esposti, il lodigiano non è affatto disposto ad ammettere il superamento dell'anarchismo e il travasarsi dei suoi contenuti positivi entro il movimento giellista:

Gli anarchici non sono disposti a fare, in seno a G.L., la parte che il rosmarino fa nell'arrosto. Essi hanno un programma proprio, e tra i giellisti non possono cercare e trovare che scambi d'idee, imprestazione di problemi, riesame di teorie. Ma anche per questo genere di contatti i giellisti farebbero bene a rinunciare al titolo di libertari del XX secolo, anche perché non è passato un secolo da quando essi tenevano più ad ingraziarsi i liberali e i socialdemocratici che gli anarchici ottocentisti. [...] Per ora accontentiamoci di discutere, non da cattedra ai banchi, ma... al caffè: tra avversari un poco cugini ${ }^{549}$.

Verrebbe da dire che, per Berneri, una cosa è la collaborazione, ma ben altra è la confusione. Soprattutto egli tende a ricordare ai giellisti la loro solo recente consonanza col libertarismo e pertanto sostiene che verso GL possa esserci apertura, confronto e cordiale dibattito nel rispetto delle reciproche specificità. Proprio in ragione di queste ultime però egli chiede ai giellisti di non autoeleggersi «libertari del XX secolo», dato che i libertari del XX secolo vi sono e operano attivamente. Non stupisce che a dirlo sia proprio colui che forse più di tutti si è sforzato per una revisione e un aggiornamento di quest'area politica, in modo da tenerne vive le tradizioni e attuale il messaggio.

${ }^{548}$ C. Berneri, Gli anarchici e "G. e L.", «Giustizia e Libertà», II, 6 dicembre 1935., segue nota di C. R. [C. Rosselli]

${ }^{549}$ Ibid. 
Una postilla all'articolo reca la risposta di Carlo Rosselli, che tende a rassicurare Berneri circa il riconoscimento dell'autonomia degli anarchici, ma difende al tempo stesso il recupero del patrimonio libertario da parte di GL:

\begin{abstract}
Berneri sa che noi sempre riconoscemmo - pur senza arrivare alla sua apologetica i meriti intellettuali delle correnti socialiste anarchiche, che dominarono il movimento proletario italiano dal 1870 sin verso il ' 90 . Ma questo riconoscimento non basta a fare di noi, come tenta Berneri, degli echeggiatori inconsapevoli quanto automatici del pensiero, [...] dei Proudhon, Bakunin, Kropotkin, Malatesta, Merlino. Attenti voi, anarchici, a non creare, dopo la Bibbia marxista, un corpo biblico anarchico, al quale solo e sempre si dovrebbero rifare coloro i quali vedono nel socialismo la più alta espressione dell'idea di liberta ${ }^{550}$.
\end{abstract}

Come si vede, toni cordiali ma senza esclusione di colpi. Rosselli mette in guardia il suo amico Berneri dal rischio di voler fare della tradizione libertaria un monopolio del movimento anarchico: per il fondatore di GL la società libera va ripensata alla luce dei nuovi problemi imposti dai mutamenti storici, pertanto il lascito dei «Maestri» può essere prezioso, ma inevitabilmente si riferisce a un altro contesto e le loro soluzioni pratiche non risultano dunque più idonee:

Le forme e le formule che si addicevano agli artigiani del Giura o ai mugik della Russia o ai braccianti del Beneventano non si addicono evidentemente agli operai della Fiat e di tutta la grande industria moderna. I mercati nazionali e mondiali chiedono ben altra coordinazione e disciplina dei mercati locali del secolo scorso. La radio, l'aeroplano, la grande stampa, la velocissima circolazione delle cose e delle idee rendono sempre più debole e vacuo ogni federalismo solo o soprattutto territoriale; mentre la potenza degli Stati totalitari e delle armi moderne costringono a concepire in modo ben altrimenti complesso i processi rivoluzionari e $\mathrm{i}$ fatti insurrezionali ${ }^{551}$.

Inconsapevolmente Carlo Rosselli con questo passaggio non fa in realtà che riferirsi proprio all'azione che il Berneri ha intrapreso per anni in seno al movimento anarchico, rilevando l'insufficienza dei vecchi schemi, del dogmatismo e del tradizionalismo degli anarchici di fronte al mondo contemporaneo. La necessità di valorizzare la tradizione libertaria, di trovarle una collocazione pratica e concreta nell'ora era un'esigenza che il lodigiano aveva avvertito in tutto il corso della sua formazione politica. Inoltre Carlo Rosselli, a proposito di questioni contingenti, avverte la necessità di una collaborazione nella prospettiva di un'unità comune contro il fascismo:

[...] unità che a nostro avviso deve comprendere comunisti, socialisti, giellisti, anarchici, repubblicani avanzati, su una piattaforma che escluda per ora ogni

\footnotetext{
${ }^{550}$ Nota di C. R. [C. Rosselli] a C. Berneri, Gli anarchici e "G. e L." cit.

${ }^{551}$ Ibid.
} 
problema di concreta successione e potere. Alleanza Rivoluzionaria Italiana; e non governo di Fronte Popolare ${ }^{552}$.

Per Rosselli questa è una soluzione per «l'oggi», ma si tratta di una scelta che determinerà anche l'avvenire rivoluzionario, poiché, se i libertari non collaboreranno intervenendo attivamente, sarà più probabile un degenerare del nuovo ordine verso nuove forme di passatismo e dispotismo statale. Pertanto, sostiene il fondatore di GL, le forze rivoluzionarie dovranno qualificarsi e organizzarsi in base ad affinità sostanziali circa le prospettive postrivoluzionarie:

Non è difficile prevedere sin d'ora - a meno di revisioni profonde e augurabili da parte comunista - la futura possibile linea di frattura delle forze rivoluzionarie. La frattura avverrà presumibilmente in relazione alle antitesi: dittatura-autonomie, socialismo o comunismo dispotico centralizzatore-socialismo o comunismo democratico federalista liberale. Guai se i fautori di un socialismo liberale e libertario saranno divisi in dieci gruppi e sottogruppi; guai se non sapranno disciplinarsi e organizzarsi solidamente. [...] Mentre noi staremo a disputarci entro che limiti debba contenersi un potere centrale, altri faranno di questo potere centrale la macchina inesorabile che tutti ci schiaccerà ${ }^{553}$.

Rosselli, come già aveva in tempi non sospetti fatto Berneri, auspica una piattaforma delle forze libertarie, onde evitare che la rivoluzione antifascista possa prendere pieghe centralistiche. I principi assoluti sono ritenuti pericolosi fattori di debolezza: solo una franca collaborazione che ruoti intorno a dei principi cardine può costituire una valida alternativa nel campo dell'antifascismo. La palla passa dunque agli anarchici stessi:

Ecco il problema, il vostro problema, socialisti anarchici: esaminare se vi convenga, per mantenervi fedeli all'assoluto libertario, conservarvi anche domani in setta a parte; oppure se non vi convenga concorrere, nell'interesse essenziale degli ideali che sono cari, a dar vita in Italia al nuovo grande libero movimento socialista italiano, condividendone coraggiosamente sin dall'inizio le corresponsabilità e i rischi tanto alla base quanto al centro ${ }^{554}$.

\section{Il confronto tra Berneri e Rosselli su federalismo e autonomie}

Dopo aver analizzata la bozza di carta costituzionale degli anarchici e la risposta di Rosselli, sembra proprio di poter affermare che la volontà di costituire una piattaforma fosse condivisa bilateralmente ${ }^{555}$. I due movimenti avvertono il pericolo

\footnotetext{
${ }^{552}$ Ibid.

${ }^{553}$ Ibid. I corsivi sono originali.

${ }^{554}$ Nota di C. R. [C. Rosselli] a C. Berneri, Gli anarchici e "G. e L." cit.

${ }^{555} \mathrm{La}$ ricostruzione biografica di De Maria ha il merito di mettere bene in luce come, negli ultimi mesi del 1935, Berneri si fosse impegnato all'interno dei circoli parigini di GL, nel tentativo di giungere all'auspicata unità d'azione antifascista tra giellisti, repubblicani dell'ARS, anarchici, socialisti massimalisti e comunisti dissidenti. Vedi C. De Maria, Camillo
} 
dell'isolamento, la necessità di agire e di imprimere una direzione chiara agli eventi rivoluzionari. Certo, pare di poter cogliere nelle parole del giellista la volontà di fare da guida, o quanto meno da ago della bilancia, del «nuovo grande libero movimento socialista italiano» e probabilmente era questo l'atteggiamento che meno di tutti predisponeva Berneri, e anarchici in generale, verso questa unificazione delle forze con GL. L'assorbimento paventato prima da Consiglio, e poi dal lodigiano, deve riferirsi proprio a questo, alla paura di subire una nuova egemonia all'interno del nuovo "movimento libertario" da parte dei «libertari del XX secolo». Tuttavia le prospettive presentano prossimità in più di un punto, come rivela una seconda lettera di Berneri al settimanale giellista, Discorso sul federalismo e l'autonomia, pubblicata il 27 dicembre 1935.

Lo scritto ruota ancora intorno alla questione delle alleanze e del "programma minimo":

Caro R.,

il nostro problema essenziale in rapporto al nuovo ruolo di comunisti libertari in seno alla rivoluzione italiana, è quello di scegliere tra l'integralismo tradizionalista e un possibilismo che, pur mantenendo fisso lo sguardo alla stella polare dell'Idea, ci permetta d'incunearci fecondamente nella linea di frattura delle forze rivoluzionarie. L'antitesi che mi pare non presumibile, come tu dici, bensì inevitabile, sarà: comunismo dispotico centralizzatore o socialismo federalista liberale.

Dal 1919 in poi non mi sono stancato di agitare in seno al movimento anarchico il problema di conciliare l'integralismo educativo e il possibilismo politico, osando sostenere polemiche e contraddittori con i più autorevoli rappresentanti del movimento ${ }^{556}$.

Berneri sostiene che nel movimento anarchico non esista alcun pericolo che sorga una sorta di Scolastica, ma semplicemente una prevalenza di correnti tendenzialmente dottrinarie. Per far meglio comprendere all'amico l'agitarsi di fermenti di rinnovamento entro l'anarchismo, il lodigiano fa riferimento al lascito intellettuale di Salvemini, loro comune maestro, parlando di

impulsi novatori, e alla propaganda generica, tradizionalista, tendenzialmente dottrinaria sta subentrando ovunque un problemismo...salveminiano precursore e nuncio di programmi aderenti a questa e a quella situazione rivoluzionaria ${ }^{557}$.

La possibilità di un dialogo al di là delle pregiudiziali è ritenuto da Berneri possibile:

Berneri cit., pp. 84-88.

${ }^{556}$ C. Berneri, Discorso sul federalismo e l'autonomia, «Giustizia e Libertà», II, 27 dicembre 1935, segue nota di C. R. [C. Rosselli]. I corsivi sono originali.

${ }^{557}$ Ibid. 
Dato che tu e gli altri dirigenti di G.L. siete persone colte, mi pare che la discussione possa essere impostata non sui residui tradizionalisti dell'anarchismo bensì su quel che di vivo, ossia di attuale e razionale, voi vedete nell'anarchismo contemporaneo. Noi e voi abbiamo di fronte il problema di come imprimere alla rivoluzione italiana un indirizzo autonomista in politica e socialista-liberista in economia ${ }^{558}$.

Si noti come il lessico politico del lodigiano tenda a muovere passi sostanziali verso quello giellista, in vista di una possibile condivisione di programma: non si parla più di «socialismo libertario» ma di «socialismo liberale di impronta federale». Tuttavia Berneri richiede un chiarimento in merito al programma di GL sul punto delle autonomie in quanto, dopo l'esperienza della Rivoluzione russa, confessa di dare importanza relativa ai programmi:

I movimenti politici navigano per forza di venti e l'apriorismo razionalista dei programmi è quasi sempre destinato a dissolversi a contatto dell'irrazionale, ossia della storia in atto. [...] Il giellismo che è, attualmente, in molte sue formule e in molti suoi atteggiamenti, vicino all'anarchismo, può domani allontanarsene in una situazione di compromesso a dispetto dei suoi dirigenti e di parte dei suoi quadri. Non vi attribuisco tenebrose manovre, ma non considero il vostro movimento abbastanza omogeneo nella sua formazione e abbastanza elaborato nel suo programma per rinunciare a riserve attuali e a preoccupazioni per l'avvenire ${ }^{559}$.

Insomma, l'indeterminatezza generale del giellismo non predispone bene il professore anarchico verso un accordo o un'unità d'azione. Sarebbe necessario a tal proposito una chiarificazione in merito al federalismo di GL che Berneri tende a considerare piuttosto come autonomismo unitario «a colorazione federalista legalitaria», intendendo con ciò un sistema di decentramento atto ad alleggerire la presenza dello Stato ma contemporaneamente a garantirne il predominio politico. Pur mettendo da parte dunque gli apriorismi, Berneri aveva colto che quello di Rosselli era sì «meno Stato», ma rimaneva pur sempre uno Stato centrale, secondo una concezione di federalismo tradizionale. Ciò si intende ancora meglio leggendo ciò che l'anarchico scrive in merito al «federalismo legalitario» e alle sue differenze con quello libertario:

L'autonomismo unitario abbraccia tutti i sistemi di decentramento atti ad alleggerire lo Stato nel campo delle sue attività amministrative ma atti altresì a garantire al governo centrale il predominio politico. [...]

Il federalismo, senza tenere conto di quello neo-guelfo, ormai superato, è autonomista-legalitario e autonomista-libertario. Il federalismo legalitario è essenzialmente repubblicano (Ferrari, Cattaneo, Rosa, Bovio, Zuccarini e La Critica politica, ecc.) e non è, in sostanza, che una integralista concezione democratica dello Stato. Nel campo socialista fu del tutto singolare la propaganda federalista del Salvemini. Il federalismo libertario (Pisacane, Bakunin, Cafiero, Malatesta, Fabbri,

\footnotetext{
${ }^{558}$ Ibid.

${ }^{559} \mathrm{C}$. Berneri, Discorso sul federalismo e l'autonomia cit. Il corsivo è originale.
} 
ecc.) si è frazionato in tre correnti principali: una riallacciantesi al comunalismo kropotkiniano, una sindacalista, una sovietista ${ }^{560}$.

Nella prospettiva berneriana, GL resta quindi tra l'autonomismo unitario, federalismo repubblicano e federalismo libertario. Ora, data l'indeterminatezza programmatica del movimento, Berneri ritiene che la prima componente potrebbe trionfare in caso di una restaurazione liberale e democratica, o di un trionfo dei comunisti, dei socialdemocratici o dei repubblicani. La seconda componente potrebbe invece prevalere in caso di rivoluzione, ove i «partiti autoritari» non abbiano la possibilità di egemonizzare il movimento.

Il lodigiano passa dunque a una disamina abbastanza cinica del possibile opportunismo politico che il giellismo potrebbe assumere:

Il giellismo [...] mi pare destinato ad essere girondino (federalista) di fronte all'unitarismo giacobino, qualora questi si sia reso, o sia per rendersi, padrone dello Stato, o giacobino (autonomista unitario) se la rivoluzione l'abbia portato ad un ruolo governativo. Nel primo caso c'incontreremo; nel secondo caso c'incontreremo egualmente, ma come avversari ${ }^{561}$.

Per un'autentica posizione socialista libertaria sarebbe necessario rinunciare al ruolo governativo e a radicarsi nella rivoluzione permanente, ma soprattutto acquisire una prospettiva federale in cui

i comuni, i sindacati, i consigli, i comitati, alla base, e le assemblee (regionali e nazionali) e le direzioni generali, al vertice, vengono, almeno in teoria, a sostituire lo Stato, delineando un sistema politico in cui al governo degli uomini subentra l'amministrazione delle cose. Questo sistema è, per Proudhon, l'Anarchia. E lo è anche per tutti i socialisti libertari ${ }^{562}$.

Ecco che Proudhon, recuperato in qualche modo dal giellismo, arriva a "presentare il conto". Dopo aver aleggiato, più o meno in penombra, nelle idee fondamentali del movimento giellista, ecco tornare il socialista francese a dirimere le nebbie ideologiche in merito al carattere libertario e e federalista di GL. Padre dell'anarchismo e pioniere del federalismo libertario, la sua figura assume il ruolo di una cartina tornasole attraverso cui valutare le reali intenzioni programmatiche del movimento di Carlo Rosselli. Berneri qui dimostra, al di là del suo anarchismo critico e sui generis, di non spingersi affatto fino alla totale eresia o al superamento dell'anarchismo, ma anzi di avere ben chiari i punti cardinali e la «stella polare dell'Idea» secondo i quali muoversi nel mare magnum dell'antifascismo. Aperto al dibattito, al confronto, volto all'aggiornamento del suo stesso movimento, egli resta fino alla fine sostanzialmente anarchico e in quanto tale legato a quella lezione

${ }^{560} \mathrm{C}$. Berneri, Discorso sul federalismo e l'autonomia cit. I corsivi sono originali.

${ }^{561}$ Ibid. I corsivi sono originali.

${ }^{562}$ Ibid. 
proudhoniana che per i giellisti resta invece sullo sfondo del loro stagliarsi rispetto alla sinistra marxista italiana.

Se è vero che Proudhon, per la natura complessa e controversa dei suoi testi, poteva prestarsi a diverse letture a seconda della sensibilità degli interpreti, pare di poter affermare che è altrettanto vero che la sua opera contenga una sostanza di fondo con cui presto o tardi bisogna confrontarsi: il suo profondo antiautoritarismo, la sua lotta senza quartiere allo Stato come centralismo dispotico e il suo federalismo teso ad annullarne o indebolirne la presenza ne fanno a tutti gli effetti un libertario. Tant'è vero che in questa chiosa di Berneri, in quello che è l'ultimo intervento nel dibattito sul federalismo con GL, la citazione del francese pare proprio finalizzata a mettere un punto, a segnare una linea di confine insuperabile. Non si può essere libertari e continuare allo stesso tempo a concepire lo Stato come ambito istituzionale di governo, anche se riformato; se si è libertari, allo Stato inteso come rapporto gerarchico governanti-governati si deve voler sostituire un rapporto più equilibrato e orizzontale, che dia valore e che lasci spazio al massimo possibile di organi autonomi. Proprio secondo la lezione di quel Proudhon che aveva insegnato ad anarchici e libertari a sentirsi e a chiamarsi tali ${ }^{563}$.

Ad ogni modo, per perentoria che possa sembrare la marcatura della distanza tra federalismo giellista e federalismo libertario da lui operata, Berneri non è certo intenzionato a sbattere la porta in faccia a Carlo Rosselli, riconosce anzi che la sua opinione sulle idee federali di G. L. sia «scarsamente documentata», e aggiunge:

Forse studiando tutto quanto avete scritto sull'argomento sarei diversamente disposto. Ma dubito che sarei del tutto soddisfatto, anche perché ho la presunzione di avere, sul federalismo, delle idee personali, per quanto riguarda l'Italia ${ }^{564}$.

In altre parole, l'anarchico lodigiano non ha piena contezza dell'ampio e ricco dibattito svoltosi nei "Quaderni di Giustizia e Libertà», ma c'è da credere che anche a fronte di una conoscenza maggiormente approfondita la sua opinione non sarebbe poi mutata più di tanto. D'altronde, persino il suggestivo auspicio formulato da Leone Ginzburg e Carlo Levi «creare uno Stato coi mezzi dell'anarchia» ${ }^{565}$ continuava ad avere lo Stato come orizzonte di riferimento: lo spirito libertario andava suscitato per poi essere negato.

Il dialogo è insomma difficile, ma di certo non è tra sordi. Deve pensarla così anche Rosselli, dal momento che la sua nota all'intervento berneriano si apre così:

\footnotetext{
${ }^{563}$ P.-J. Proudhon, Qu'est-ce que la propriété cit., p. 278: «Che cosa siete dunque?» «Io sono anarchico». «Ho capito: fate della satira contro il governo». «In alcun modo: quella che avete appena udita è la mia professione di fede seria e meditata: benché molto amico dell'ordine, io sono, in tutta la forza del termine, anarchico».

${ }^{564}$ C. Berneri, Discorso sul federalismo e l'autonomia cit.

${ }^{565}$ M. S. [C. Levi e L. Ginzburg], Il concetto di autonomia nel programma di "G.L.", «Quaderni di Giustizia e Libertà», n 4, settembre 1932, p. 9.
} 
Berneri conferma autorevolmente la nostra interpretazione dell'anarchismo collettivista come socialismo federalista liberale e riconosce la necessità che gli anarchici abbiano a prendere posizione domani in una concreta situazione rivoluzionaria per far trionfare soluzioni di libertà su soluzioni di dittatura.

È un primo punto, ma è il punto decisivo. I socialisti e i comunisti anarchici sono numerosi in Italia; [...] e, se sapranno domani accettare le responsabilità non solo dell'azione (il che è certo) ma anche della ricostruzione, potranno esercitare una notevole influenza contribuendo anche ad evitare le deviazioni di altri movimenti a loro affini. Giacché da che cosa derivano, o potrebbero derivare, quelle deviazioni? Dal fatto che su una posizione di intransigente difesa dei principi di autonomia e di libertà non si sono trovate sinora, nella classe operaia e contadina, forze abbastanza solide per contenere le tendenze dittatoriali accentratrici. [...]

Quindi, socialisti e comunisti libertari, se volete vincere domani o almeno non soccombere bisogna che vi prepariate sino ad ora ad opporre alle concrete soluzioni dittatoriali una concreta, attuabile soluzione socialista federalista liberale. La quale, beninteso, non spranghi le porte a progressi ulteriori ${ }^{566}$.

Inconsapevolmente, Carlo Rosselli sta facendo a Berneri le stesse proposte che quest'ultimo ha fatto al movimento anarchico durante l'esilio: lotta antifascista e rivoluzione libertaria come binomio inscindibile, programma concreto per la ricostruzione in senso autonomista e federalista, e azione politica atta ad arginare il ritorno di soluzioni autoritarie e centraliste.

Più avanti nella sua nota, Rosselli si prende dello spazio per «fissare qualche idea intorno al nostro socialismo federalista liberale» e lo schematizza in sei punti dichiarando:

1) che per G. L. il federalismo politico territoriale è un aspetto e una applicazione del più generale concetto di autonomia a cui il nostro movimento si richiama: cioè di libertà positivamente affermata per i singoli, gruppi, in una concezione pluralistica dell'organizzazione sociale;

2) che la regione storica, utile ai fini politici amministrativi, può diventare mortifera a fini economici e culturali, la regione agricola non coincidendo con la regione storica, la regione industriale variando da industria a industria [...]. Perciò, anche in materia di regioni, pluralismo, elasticità;

3) che, specie dopo il fascismo, anziché rivalutare la patria regionale bisognerà sforzarci di recuperare o allargare la patria nazionale in cui si asfissia, facendola coincidere con la nozione di patria umana o umanità, espressione di valori essenziali comuni a tutti gli uomini [...];

4) che gli organi vivi dell'autonomia non sono gli organi burocratici, indiretti, in cui l'elemento coattivo prevale, ma organi di primo grado, diretti, liberi o con un alto grado di spontaneità, alla vita dei quali l'individuo partecipa direttamente o che è in grado di controllare. Quindi il comune, organo territoriale che ha in Italia salde radici e funzioni; il consiglio di fabbrica e di azienda agricola, organo o uno degli organi dei produttori associati; la cooperativa, organo dei consumatori; le camere del lavoro, $i$ sindacati, le leghe, organi di protezione e di cultura professionale; i

${ }^{566}$ Nota di C. R. [C. Rosselli] a C. Berneri, Discorso sul federalismo e l'autonomia cit. I corsivi sono originali. 
partiti, i gruppi, i giornali, organi di vita politica; la scuola, la famiglia, i gruppi sportivi, i centri di cultura e le innumerevoli altre forme di libera associazione, organi di vita civile;

5) che è partendo da queste istituzioni nuove o rinnovate, legate fra loro da una complessa serie di rapporti, e la cui esistenza dovrà essere presidiata dalle più larghe libertà di associazione, di stampa, di riunione, di lingua, di cultura, che si arriverà a costruire uno Stato federativo orientato nel senso della libertà, cioè una società socialista federalista liberale;

6) che il concetto di autonomia deve valere non solo per il domani ma anche per oggi; non solo per la ricostruzione ma per la lotta che dovrebbe condursi secondo questi criteri $[\ldots]^{567}$.

È sorprendente notare quanti punti di contatto presenti questa esposizione riassuntiva del federalismo giellista con il "programma minimo" anarchico promosso da Berneri e temporaneamente condensatosi negli indirizzi della Relazione $C$ e nel testo della Costituzione. I punti 4 e 5 in particolare sembrano riflettere, seppur sinteticamente, in tutto e per tutto le idee berneriane di partecipazione diretta e di controllo da parte degli individui e di una sorta di repubblica dei consigli federalista e libertaria che risulti dai legami tra i vari organi autonomi, in una sorta di processo ascendente. Il secondo punto è a sua volta oltremodo vicino all'elaborazione dell'anarchico lodigiano, in particolare per quel che riguarda il fatto che il federalismo politico-territoriale debba essere completato e arricchito dal federalismo economico e sociale: entrambi hanno indicato il Comune e gli organi dei produttori e dei consumatori (consigli di fabbrica, cooperative, sindacati, etc.) quali cellule-base della nuova organizzazione. Infine, la necessità di fare dell'autonomismo non solo la cifra della ricostruzione, ma anche della stessa battaglia antifascista.

Non c'è insomma da stupirsi se sulla carta stampata il confronto su questo tema tra Berneri e Rosselli si arresta qui, in quanto è facile immaginare che - anche se consapevoli delle specifiche e diverse posizioni - entrambi abbiano potuto cogliere la possibilità di avviare uno scambio politico e un lavoro organizzativo comune tra movimento giellista e anarchici. Insomma, dalle parole bisognava passare ai fatti.

\section{Torna Salvemini...?}

C'è una terza voce nel dibattito tra Berneri e Rosselli, una terza voce di cui le ricostruzioni storiche hanno finora scarsamente tenuto conto. L'unico articolo del terzo interlocutore - che si firma Giesse e potrebbe essere con ogni probabilità proprio Gaetano Salvemini ${ }^{568}$ - compare in effetti molto tardi, nel numero del 10

${ }^{567}$ Ibid. I corsivi sono originali.

${ }^{568}$ L'ipotesi è stata proposta da Malandrino, vedi C. Malandrino, Socialismo e libertà cit., pp. 129-135, ed è stata accolta anche da Lacaita, cfr. C. Lacaita, Salvemini e il federalismo, in G. Pescosolido (a cura di), Gaetano Salvemini (1873-1957). Ancora un riferimento. Atti del convegno di studi tenutosi a Roma l'11 e il 12 dicembre 2007 in collaborazione con la Fondazione "Ernesto Rossi e Gaetano Salvemini", Lacaita, Manduria-Bari-Roma, 2010, pp. 69-71. 
gennaio $1936^{569}$, quando lo scambio sul federalismo tra l'anarchico e il giellista si è di fatto concluso e non ha conosciuto ulteriori sviluppi sulle colonne di «Giustizia e Libertà».

L'autore interpella direttamente $\mathrm{i}$ due protagonisti del dibattito e li esorta a rifuggire le astrattezze:

Agli amici Berneri e C. R.

Domanderei qualche schiarimento in merito al «Federalismo» di cui avete discusso nel giornale del 27 dicembre scorso.

Non pago del desiderio ideale di una vaga libertà, vedo con piacere che si precisi il generale bisogno di vivere e di agire senza soverchi impacci, in una formula più conveniente $\mathrm{e}$ più aderente alle possibilità moderne.

Tutto ciò che va sotto il nome di «autonomia» e di «federalismo» attira la mia attenzione. Non sempre, però, la mia curiosità si trova soddisfatta, perché molti costruiscono dei sistemi astratti arbirarii, anziché applicarsi ad aggiustare od a rifare e numerare i pezzi che devono rimpiazzare quelli logori e cattivi nel meccanismo amministrativo e politico italiano ${ }^{570}$.

E più avanti entra nello specifico dei problemi che l'autonomismo potrebbe portare:

Per l'autonomia che si dovrebbe praticare fin d'ora nei gruppi - giusta - bisogna tuttavia guardarsi dall'allentare troppo i legami tra centro e periferia.

$[\ldots]$

Domanderei che si confrontassero le proprie convinzioni federalistiche con i principi codificati nelle costituzioni di alcuni Stati (Svizzera, Stati Uniti) e ci si provasse a cementarli al fuoco di una concreta realtà, per vedere se la medaglia non abbia un rovescio non conforme alle proprie speranze $\mathrm{e}^{571}$.

Il tono dell'articolo sembra a tutti gli effetti caratterizzato dall'atteggiamento intellettuale concretista e problemista di Salvemini, specialmente nel rimandare all'esame di esempi di Stati organizzati secondo un sistema federale. Giesse si mostra soprattutto preoccupato che il modello ideale si misuri con la realtà economica del mondo moderno:

È assurdo e impossibile frazionare (e il decentramento è sempre una finestra aperta verso il frazionamento) le ferrovie, il telegrafo, la radio, l'aviazione, le sorgenti d'acqua, l'elettricità, i porti. C'è bisogno invece di andare avanti. [...]

C'è d'altra parte una tendenza opposta che esce dal seno stesso delle tendenze accentratrici delle industrie moderne: il decentramento urbano portato dai mezzi di comunicazione più leggeri, e il decentramento industriale dovuto all'elettricità che

\footnotetext{
${ }^{569}$ Giesse [G. Salvemini], Federalismo superato, «Giustizia e Libertà», III, 10 gennaio 1936, segue nota di C. R. [C. Rosselli].

${ }^{570} \mathrm{Ibid}$.

${ }^{571}$ Giesse [G. Salvemini], Federalismo superato cit.
} 
pare facciano rivivere un artigianato numeroso, quantunque in appendice alla centralizzata industria elettrica e alla mercé delle banche.

Insomma molte ragioni fanno ritenere che il problema del centralismo o del federalismo inteso alla vecchia maniera sia superato. Per il resto, sembra una questione più tecnica che politica, da delegare a speciali commissioni di studio al momento opportuno, e non da decidersi nei comizi ${ }^{572}$.

Troviamo la prima parte di questa citazione particolarmente importante e significativa: si ricorderà che su questi ed altri servizi o attività di interesse nazionale la Costituzione berneriana prevedeva un gran numero di «direzioni nazionali», riconoscendo dunque la necessità di non trasformare l'autonomia in una spinta centrifuga che avrebbe potuto portare a un'eventuale paralisi del paese. Sarebbe una nuova conferma dell'influenza esercitata dal pensiero salveminiano sull'elaborazione politica di Berneri, un portato di lungo periodo fecondamente innestatosi all'interno del suo federalismo libertario.

Giesse pone infine una suggestione, una nuova questione da affrontare per chiunque voglia riflettere ed elaborare una proposta federalista e autonomista al passo coi tempi:

Piuttosto, è tutta la questione del regime rappresentativo, che è oggi in giuoco, ma sotto un aspetto, diremo così, verticale, portato dal sindacalismo.

Comunque, risolto il problema delle classi, si scopre sin d'ora il contrasto tra le varie categorie della produzione di fronte alla rappresentanza generale della società.

Poiché il peso di dette categorie varia per qualità e quantità di fronte a tutta la popolazione, occorre equipararle con opportune e urgenti disposizioni onde evitare nuove forme di oppressione e di privilegio ${ }^{573}$.

In altre parole, il problema è quello del rapporto tra rappresentanza politica e rappresentanza professionale, del peso che ambedue avranno a livello locale e nazionale e dei rispettivi contrappesi. Si tratta di una tematica che ci sembra confermare ulteriormente la possibilità che dietro lo pseudonimo Giesse si celi Gaetano Salvemini: accanto alla formulazione tecnica di un federalismo ascendente che dai municipi risalisse progressivamente fino a un Parlamento nazionale con specifiche attribuzioni, anche il tema del federalismo sociale e della rappresentanza degli interessi era stato al centro del dibattito de «L'Unità» e dai gruppi della Lega democratica tra 1919 e 1920, dibattito in cui erano emerse idee di riforma dello Stato in senso autonomista attraverso l'istituzione di specifici organi di rappresentanza professionali e di categoria ${ }^{574}$.

\footnotetext{
${ }^{572}$ Ibid.

${ }^{573}$ Ibid.

${ }^{574}$ Vedi Fabio Grassi, Salvemini e l'ultima «Unità», in G. Cingari (a cura di), Gaetano Salvemini tra politica e storia, Laterza, Roma-Bari, 1986, pp. 329-353, e F. Grassi Orsini, Salvemini e il federalismo, in M. Degl'Innocenti (a cura di), Gaetano Salvemini e le autonomie locali cit., pp. 91-96.
} 
Ci pare significativo che a mettere un punto sul dibattito sia stato, con ogni probabilità, Gaetano Salvemini, comune fonte di ispirazione per Rosselli e per Berneri: egli sembra intervenire ancora una volta per rimarcare la necessità di fuggire le astrattezze, di andare a fondo nello studio dei problemi senza accontentarsi di formule semplici o di principi da opporre ad altri principi, di ricercare soluzioni concrete, attuabili, immediatamente percorribili. A rimarcare, in altre parole, quel lascito intellettuale che egli aveva consegnato ad ambedue.

\section{Verso un epilogo: ultimi episodi di riflessione federalista}

Dagli ultimi mesi del 1935 e fino a circa la prima metà del 1936, Berneri si confronta soprattutto con GL e i repubblicani dell'ARS: sono queste le due nuove forze che sembrano poter dar vita a un nuovo socialismo democratico, repubblicano, federalista e classista, passibile di arricchirsi anche di contenuti libertari. L'anarchico lodigiano sarà particolarmente attivo in questo arco di tempo nel tentare in tutti $\mathrm{i}$ modi di trovare un convergenza tra il suo movimento, i giellisti e i repubblicanosocialisti, ma anche i socialisti massimalisti e i comunisti dissidenti (bordighisti e trotzkisti). Sarebbe stato il realizzarsi dell'alternativa antifascista da lui così a lungo rincorsa, e gli anarchici - già al lavoro per approfondire e ampliare il proprio "programma minimo" - avrebbero potuto giocarvi un ruolo importante, contribuendo a determinarne uno sviluppo in senso libertario. Il dialogo e i contatti saranno fitti, ma sempre difficili e comunque mai tali da portare al concretizzarsi della convergenza di forze che avrebbe desiderato Berneri ${ }^{575}$.

L'apice sarà toccato a una riunione parigina di GL nel giugno 1936, in cui Carlo Rosselli propone di esaminare la situazione dell'antifascismo

allo scopo di vedere se può trovarsi un "nuovo antifascismo" [...] una piattaforma comune, anche una collaborazione di educazione politica senza pregiudizio per l'avvenire che potrà dare anche vita ad accordi più precisi. Scopo non è di arrivare, ma solo di vedere se possibile trovare questo accordo ${ }^{576}$.

Nel corso della riunione, Rosselli presenta tre proposte in questo senso:

$1^{\wedge}$ - formare una specie di comitato di collegamento che riunisca persone di varie correnti e senza allontanarle dai loro partiti.

$2^{\wedge}$ - fare, con mezzi adatti, un'inchiesta sulla vera situazione in Italia allo scopo di trovare le crepe attraverso le quali fare penetrare il "nuovo antifascismo".

${ }^{575}$ Gli eventi, gli incontri e e gli scambi epistolari che hanno caratterizzato questa fase sono stati ben ricostruiti da De Maria, vedi C. De Maria, Camillo Berneri cit., pp. 88-102.

${ }^{576}$ Informazione confidenziale, 5 giugno 1936, in ACS, Min. Int., Dir. Gen. PS, Div. Pol. Polit., fascicoli per materia, b. 127, fsc. 1d, Giustizia e Libertà, Parigi. 
$3^{\wedge}$ - creare insieme un nuovo centro di cultura, una specie di università proletaria che dia una cultura antifascista ed obiettiva attraverso un programma di studi e di lezioni ${ }^{577}$.

Forse perché disilluso dopo i continui nulla di fatto, forse perché ancora convinto della necessità di studiare a fondo le problematiche inerenti alla rivoluzione e le relative soluzioni concrete, forse perché l'idea di un centro di cultura pensato per questo scopo gli poteva evocare il ricordo e l'esperienza del circolo salveminiano dei tempi universitari, Berneri si dirà concorde soltanto sul terzo punto. In questo contesto, prima che la fiammata rivoluzionaria della Spagna richiami all'azione, si collocano le ultime, significative riflessioni berneriane sul tema del federalismo.

\section{Le chiusure del movimento anarchico}

Berneri e quella parte di anarchici disponibili e aperti verso il dialogo con forze politiche affini devono fare i conti con un movimento organizzato o con esponenti antiorganizzatori che sono di tutt'altro avviso. $\grave{E}$ significativo rilevare che tra dicembre 1935 e gennaio 1936 - vale a dire nel medesimo periodo in cui si sviluppa il confronto tra Berneri e Rosselli su «Giustizia e Libertà» e alle riunioni gielliste l'Unione Anarchica Italiana (ricostituitasi a Parigi nel 1927) diffonda tre circolari intitolate "Contro il movimento 'Giustizia e Libertà"; queste circolari, di cui sono conservate le trascrizioni operate dalla Divisione Polizia Politica, sono state da noi consultate presso l'Archivio Centrale dello Stato $^{578}$ e non risultano essere mai state prese in considerazione né da alcuno storico del pensiero politico né da alcuno storico del movimento anarchico. Fabrizio Giulietti, nel suo pur documentatissimo lavoro sul movimento anarchico nella lotta contro il fascismo, ha invero parlato di una circolare che dissuadeva gli anarchici dall'avvicinarsi a GL, tuttavia non solo non ha omesso un'indicazione precisa della fonte, ma ha parlato di un testo diffuso dalla Federazione Anarchica Profughi Italiani (FAPI) ${ }^{579}$, non della UAI. Quest'ultima si era in effetti trasformata dapprima in Unione Comunista Anarchica Profughi Italiani (UCAPI) nel 1930, quindi in FAPI nel 1933; potrebbe dunque essere che la Polizia Politica stesse continuando a identificare la FAPI con la UAI, mantenendo quest'ultima sigla. Resterebbe tuttavia un'incongruenza, poiché le circolari, come detto, sono tre e non una sola, come riportato da Giulietti; ad ogni modo, nessuna ricerca ha finora citato neanche un estratto di questi documenti, che noi dunque citiamo e riportiamo qui per la prima volta.

La prima circolare è datata 16 dicembre 1935 e mette in guardia i compagni dai militanti giellisti, ritenuti elementi inaffidabili ai fini rivoluzionari:

\footnotetext{
${ }^{577}$ Ibid.

${ }^{578}$ ACS, Min. Int., Dir. Gen. PS, Div. Pol. Polit., fascicoli per materia, b. 124, fsc. 8, Rapporti fra Giustizia e Libertà e anarchici.

${ }^{579}$ Vedi F. Giulietti, Il movimento anarchico italiano nella lotta contro il fascismo cit., pp. 137-138.
} 
La generosità di quanti, come [i] borghesi di "Giustizia e Libertà", aspirano a governarvi, consiste nel promettervi e restituirvi in una forma o un'altra una piccola parte di quel che voi avete lor dato.

Ecco infatti gli estensori del manifesto impegnare il governo monarchico o repubblicano borghese in ogni caso, che secondo i loro desideri dovrebbe raccogliere l'eredità del Fascismo, "al trasferimento alle organizzazioni operaie e contadine di tutte le proprietà del partito e delle organizzazioni fasciste" e al "sequestro dei beni di tutti i responsabili della dittatura".

[...] Ma ricordate compagni lavoratori, che il Governo della successione non potrà darvi, non potrà restituirvi, per essere più esatti, codesta ricchezza se non a condizione che voi lo mettiate [...] in grado di toglierla alle organizzazioni ed ai capi del Fascismo, che [...] oggi la detengono.

Il Governo di domani non ha più che non lo abbia quello di oggi il diritto di governarvi $^{580}$.

Più avanti la prima circolare incita gli anarchici a fare da sé e a non lasciarsi assorbire nelle fila di GL:

Togliete alla tirannia che oggi vi opprime e vi dissangua la complicità involontaria della vostra inerzia ed essa crollerà, negate a coloro che aspirano a succederle l'investitura del vostro suffragio, il sangue del vostro sacrificio, nelle rivolte imminenti, e Voi resterete liberi.

Espropriate per voi, pel vostro lavoro e pel vostro sudore fecondi le proprietà e le ricchezze che vi furono usurpate e voi ne potrete godere per l'avvenire tutti i benefici ${ }^{581}$.

La circolare successiva, del 24 dicembre 1935, torna a insistere sul carattere controrivoluzionario di GL, in quanto

se onestamente essi sono convinti di quanto vi promettono, il trasferimento alle organizzazioni operaie e contadine di tutte le proprietà del partito e delle organizzazioni Fasciste ed il sequestro [...] dei beni di tutti i "responsabili della Dittatura" implica l'espropriazione pura e semplice di quattro quinti almeno della ricchezza privata italiana.

[...] Ma che cosa sarebbe una rivoluzione tendente ad espropriare quattro quinti della ricchezza privata se non una rivoluzione a largo di carattere sociale? ${ }^{582}$

Questa perplessità si unisce peraltro ad altre che portano inevitabilmente i redattori a diffidare del movimento giellista, poiché

${ }^{580}$ Trascrizione della Circolare dell'Unione Anarchica Italiana "Contro il movimento 'Giustizia e Libertà'", 16 dicembre 1935, in ACS, Min. Int., Dir. Gen. PS, Div. Pol. Polit., fascicoli per materia, b. 124 , fsc. 8 cit.

${ }^{581}$ Ibid.

${ }^{582}$ Trascrizione della Circolare dell'Unione Anarchica Italiana "Contro il movimento 'Giustizia e Libertà'", 24 dicembre 1935, in ACS, Min. Int., Dir. Gen. PS, Div. Pol. Polit., fascicoli per materia, b. 124, fsc. 8 cit. 
il confessato atteggiamento antirivoluzionario, la preoccupazione emanante da ogni pagina di salvare il più che sia possibile delle vecchie istituzioni politiche, e soprattutto economiche, lasciano bene intendere che queste due clausole del programma di "Giustizia e Libertà" non hanno se non un valore demagogico $[\ldots]^{583}$.

Il punto sulla questione viene però messo in maniera perentoria con la terza circolare, datata 2 gennaio 1936:

In una parola "Giustizia e Libertà" non vi propone soltanto di conservare lo Stato con o senza monarchia, la proprietà privata del monopolio capitalistico e la conseguente schiavitù del salariato, ma anche l'ignominia del tribunale squadrista che, mutato personale, chiamerà tribunale rivoluzionario e con cui compierà le vendette dello Stato antifascista [...]

Convenite compagni lavoratori che vi si invita ad offrire il vostro consenso, la vostra opera, il vostro sacrificio per una larva di "rivoluzione" che, debellate alcune forme della dittatura Fascista, mira a conservarne intatta gran parte se non tutta la sostanza e, tolti dalla circolazione alcuni tra i suoi maggiori responsabili, a consolidare col terrore il dominio dei nuovi padroni ${ }^{584}$.

La chiusura e la diffidenza sono insomma totali da parte del movimento anarchico organizzato, che mette in rilievo tutte le contraddizioni tra $\mathrm{i}$ fermenti rivoluzionari giellisti e le sue tendenze a porsi come forza di governo e, conseguentemente, autoritaria. Berneri, lo abbiamo visto, è altresì convinto che l'atteggiamento politico di GL non sia abbastanza netto e definito, anzi ne teme soprattutto le possibili oscillazioni a seconda dell'evolversi degli eventi rivoluzionari ${ }^{585}$; eppure per l'anarchico lodigiano il movimento di «Giustizia e Libertà» non è né antirivoluzionario né demagogico, ma anzi passibile di essere uno dei migliori compagni di strada in vista di una rivoluzione antifascista di segno libertario.

L'anarchico lodigiano chiarirà i limiti e l'ampiezza della sua apertura nei confronti di GL in un articolo pubblicato su «L'Adunata dei Refrattari» del 4 aprile 1936 e intitolato Come vedo il movimento giellista ${ }^{586}$, che compare seguito da una nota redazionale di Sartin. Berneri intende rispondere alle «voci pantografanti la mia simpatia culturale per il cenacolo giellista di Parigi fino a fare di essa un inserimento», sottolineando peraltro che i militanti di GL siano stati «cordialmente

\footnotetext{
${ }^{583} \mathrm{Ibid}$.

${ }^{584}$ Trascrizione della Circolare dell'Unione Anarchica Italiana "Contro il movimento 'Giustizia e Libertà', 2 gennaio 1936, in ACS, Min. Int., Dir. Gen. PS, Div. Pol. Polit., fascicoli per materia, b. 124, fsc. 8 cit.

${ }^{585}$ Vedi supra.

${ }^{586} \mathrm{C}$. Berneri, Come vedo il movimento giellista, «L'Adunata dei Refrattari», XV, 4 aprile 1936, segue nota di M. S. [M. Sartin]. L'articolo è riprodotto in Id., Pietrogrado 1917 Barcellona 1937 cit., pp. 177-182 (col titolo Socialisti libertari e socialisti liberali), e in Id., Anarchia e società aperta cit., pp. 216-219.
} 
accoglienti nelle riunioni della sigla nonostante sia stato uno dei più insistenti e talvolta dei più aspri avversari del giellismo ${ }^{587}$. Berneri entra subito nel merito, sgombrando il campo da ogni dubbio:

Io resto avversario del giellismo, ma sono curiosamente e cordialmente attento all'attività dei giellisti che conosco: quelli di Parigi. Io mi rifiuto di considerare "diciannovisti ritardatari" dei giovani intelligenti, colti e di animo generoso nei quali non riesco a scorgere una forma mentis mussoliniana ma nei quali vedo, invece, una ferma volontà di formazione politica, il disgusto per l'improvvisazione programmatica e per la demagogia, un'appassionata ricerca di colmare le proprie lacune di cultura e di esperienza nello studio e nel contatto con elementi dei vari partiti e movimenti dell'emigrazione antifascista.

Distinguo l'opportunismo di alcuni dirigenti di G. e L. dalla ricerca della maggioranza dei giellisti di origine politica e sociale molto varia e di una generazione che si è formata le ossa in un'epoca del tutto inadatta a capire cristallizzazioni teoriche. E distinguo opportunismo agitatorio da confusionismo ${ }^{588}$.

Dopo aver messo in chiaro la propria posizione, Berneri prende a questo punto le difese dei giellisti sostenendo che:

Lo schema esotico dell'abbozzo programmatico di G. e L. non è dovuto a povertà di sintesi costruttiva bensì al profondo senso della storia e alla coscienza del ramificarsi di ogni problema in cento e più problemi, senso e coscienza che sono propri di ogni intellettuale. $[\ldots]$ Ora che cos'è un programma politico se non una sintesi escludente la precisa analisi possibile soltanto nel problemismo concreto e particolare? [...] Dove finisce la cautela dello scienziato e dove comincia l'astuzia del politico nell'opportunismo di alcuni dirigenti di G. e L.? Io non lo so. Quello che constato [...] è questo: che i repubblicani di destra sono fermi alla Repubblica del $1849 \mathrm{e}$ quelli di sinistra non sono riusciti né ad elaborare il federalismo né a sottrarsi ad un mimetismo bolscevizzante; che i socialisti unitari e quelli massimalisti non hanno un programma della rivoluzione italiana che non sia un canovaccio di richiami marxisti; che $\mathrm{i}$ comunisti dal giacobinismo alla russa non sono riusciti a trarre che un possibilismo che è più che mai "diciannovista" 589 .

In altre parole $\mathrm{i}$ giellisti condividono un atteggiamento intellettuale che Berneri conosce bene e che ha caratterizzato fortemente la sua formazione politica, tanto basta a non renderli dei demagoghi agli occhi del lodigiano. La dettagliata anatomia dell'antifascismo italiano è funzionale proprio a dimostrare questo, cioè che GL è l'unico movimento che si è posto il problema di un programma per la rivoluzione e la ricostruzione dell'Italia su basi nuove, altro aspetto che nei fatti avvicina l'anarchico lodigiano ai giellisti. Dunque è opportuno distinguere, poiché - e qui Berneri sembra parafrasare il maestro Salvemini - vi è «un possibilismo ingenuo

${ }^{587} \mathrm{C}$. Berneri, Come vedo il movimento giellista cit. Il corsivo è originale.

${ }^{588}$ Ibid. I corsivi sono originali.

${ }^{589}$ Ibid. 
come vi è un estremismo ingenuo. Tutto sta non nell'essere possibilisti od estremisti bensì nell'essere rivoluzionari intelligenti»» ${ }^{590}$.

Secondo la sintesi compiuta da Berneri, «Giustizia e Libertà»

deve invertire il rapporto tra il suo programma e il suo spirito. In un primo tempo il programma è stato più rosso di quello che non fosse l'assieme dei dirigenti, in un secondo tempo, l'attuale, l'assieme dei suoi aderenti e dei suoi dirigenti è più rivoluzionario del programma. [...] Noi siamo anarchici e come tali il dilemma è uno solo, per noi: o "Giustizia e Libertà" evolve verso il socialismo libertario fino a toccare l'ala socialista libertaria del movimento anarchico o la collaborazione rimarrà generica.

Il movimento giellista, precisando il proprio programma, subirà altre scissioni, che si delimiteranno sulla linea dello Stato e dell'Anarchia, che non è semplicemente il non-Stato bensì un sistema politico a-statale, ossia un insieme di autonomie federate ${ }^{591}$.

Sono, come si ricorderà, gli stessi termini della questione che Berneri aveva posto di fronte a Rosselli nel loro dibattito su federalismo e autonomie. Ritorna ancora una volta inoltre l'idea berneriana per la quale il realizzarsi politico dell'anarchia risulta, in ultima istanza, dal sostituirsi della federazione di autonomie locali e sociali a una gestione del potere di tipo verticistico, accentrato e autoritario, idea su cui ha a lungo insistito e che - come sappiamo - ha cominciato a delineare nei suoi contorni e nei suoi contenuti insieme ad un gruppo di compagni. A loro deve far riferimento quando, in conclusione dell'articolo, dichiara:

Il giorno in cui mi considerassi giellista vorrebbe dire: o che io da anarchico sono divenuto liberale-socialista o che il giellismo è diventato socialista libertario come lo sono io e lo sono con me altri libertari del secolo $X X$. Ci sarebbe, allora, non un'alleanza rivoluzionaria $[\ldots]$ bensì una nuova organizzazione convivente pacificamente con le altre organizzazioni libertarie, con i gruppi autonomi e con i compagni non-organizzati ${ }^{592}$.

Interessante come Berneri qualifichi se stesso e altre individualità come «libertari del secolo XX», utilizzando quella formula che Rosselli gli aveva opposto lamentando la scarsa disponibilità all'aggiornamento e all'elaborazione politica da parte degli anarchici. I nuovi libertari non sono insomma i giellisti - non adesso, non ancora, un domani forse, chissà - ma lo sono invece tutti quegli anarchici disposti a uscire dagli apriorismi, dall'ostinato aggrapparsi ai principi, dal continuo ribadire $i$ capisaldi del proprio pensiero politico senza confrontarsi e misurarsi con lo studio dei problemi e con l'elaborazione di soluzioni concrete. Sono, diremo così, quelli che hanno accettato la sifda di riflettere sulla struttura e sul fondamento di una nuova organizzazione sociale e politica, un'organizzazione federalista, autonomista e

\footnotetext{
${ }^{590} \mathrm{Ibid}$. I corsivi sono originali.

${ }^{591} \mathrm{Ibid}$. Il corsivo è originale.

${ }^{592} \mathrm{Ibid}$. Il corsivo è originale.
} 
libertaria. Sono quelli che di fronte alle questioni che impone la rivoluzione non assumono posizioni come quelle di Max Sartin che, nella lunga nota redazionale di risposta all'articolo berneriano, sposta tutta la questione sui principi.

Per il direttore de «L'Adunata dei Refrattari» la scelta è semplice, o si è autoritari o si è libertari, e i principi

professati da "Giustizia e Libertà" sono autoritari: i suoi uomini aspirano a diventare governo o sostenitori di governo, e tanto basta perché il movimento di "Giustizia e Libertà" sia inconfondibile col movimento anarchico ${ }^{593}$.

Secondo Max Sartin non c'è alcuna possibilità che GL si sviluppi in senso libertario e che possa essere, un domani, al fianco degli anarchici; di più, essa «non si trova rispetto all'anarchismo in posizione diversa da quella in cui si trovano gli altri partiti», ed è necessario stare in guardia e tener fede ai propri principi poiché «la rivoluzione sociale è soprattutto un problema di fondamenti, e bisogna stare in guardia perché il fascino della coltura non ci allontani dai principi fondamentali» ${ }^{594}$. Niente problemismo, niente concretismo, niente studio e dibattito culturale dunque, perché c'è il serio rischio di perdere di vista la giusta via da percorrere.

\section{Cattaneo completato dal sovietismo}

Il 17 marzo 1936 la Divisione Affari Generali Riservati annota che, stando a fonti fiduciarie, Berneri «avrebbe intenzione di fondare un nuovo partito a fondo anarchico ed a tale scopo ne starebbe preparando il programma» ${ }^{595}$. È chiaro che si tratta di una terminologia imprecisa o comunque da prendere con la dovuta cautela, anche perché non solo nessun anarchico - neanche Berneri, per quanto sui generis penserebbe mai di fondare un partito propriamente detto, ma addirittura il lodigiano stesso non ha mai espresso alcuna tendenza o interesse a muoversi in questa direzione.

In senso lato, tuttavia, fin dai mesi immediatamente successivi al Convegno di Sartrouville, Berneri stava effettivamente lavorando all'interno del movimento con un gruppo di compagni disponibili a muoversi su un "programma minimo"; inoltre, come abbiamo visto, qualificava questo gruppo come «libertari del secolo XX». Le riunioni sui temi del dopo-rivoluzione promosse dal Comitato organizzatore sono continuate, ne abbiamo qualche notizia dalle carte della Polizia Politica che segnalano riunioni per l'esame dei problemi del dopo rivoluzione ${ }^{596}$ e che ci sembra non facciano che confermare come la Relazione $C$ e la Costituzione fossero stati dei

\footnotetext{
${ }^{593}$ Nota di M. S. [M. Sartin] a C. Berneri, Come vedo il movimento giellista cit.

${ }^{594}$ Ibid.

${ }^{595}$ Nota della Dir. Gen. PS, AA. GG. RR., Sez. Prima, 17 marzo 1936, in ACS, Min. In., CPC, Berneri Camillo, b. 537, fsc. III.

${ }^{596}$ Vedi informazione confidenziale, 1 marzo 1936, in ACS, Min. Int., Dir. Gen. PS, Div. Pol. Polit., fascicoli per materia, b. 25, fsc. 1 cit.; e appunto della Div. Pol. Polit., 14 marzo 1936 per la Dir. Gen. PS, AA. GG. RR., Sez. Prima, in ACS, Min. In., CPC, Berneri Camillo, b. 537 , fsc. III
} 
punti di partenza, delle coordinate su cui muoversi per l'elaborazione di un programma politico per il movimento anarchico, o almeno per una parte di esso.

Berneri non ha per questo smesso di cercare esempi nella storia del pensiero politico, anzi proprio nel corso del 1936 si situa quella che è con ogni probabilità la sua ultima grande pubblicazione sul tema del federalismo: uno studio su Carlo Cattaneo, il repubblicano cui era stato introdotto da Salvemini e che aveva rappresentato a lungo un riferimento anche per la sua riflessione libertaria. Il saggio Carlo Cattaneo, federalista viene pubblicato a puntate sulla rivista «Studi Sociali» di Montevideo ${ }^{597} \mathrm{e}$, fin dall'apertura, tradisce la volontà da parte di Berneri di darne un'immagine tutta filtrata attraverso l'interpretazione salveminiana, un'immagine funzionale peraltro a sostenere quella che fino ad allora era stata anche l'impostazione della propria battaglia all'interno del movimento.

L'anarchico lodigiano sostiene che l'opera di Cattaneo

fu impostazione di problemi, concretezza di analisi, ossia preparazione di studioso e non sbandieramento di sonanti parole, positivismo e non trascendentalismo, scienza e non demagogia. Nessuna declamazione, nessun volo romantico, in quell'opera, bensì eloquenza sostenuta, pensiero cristallino, trattazione rigorosa. Egli guarda alle stelle dell'ideale, ma ancor di più alla strada della storia; e pare quasi un caso che il suo nome rimanga legato alle giornate barricadiere di Milano ${ }^{598}$.

Ancora una volta troviamo i motivi del concretismo e del problemismo, un filo conduttore che Berneri sembra stabilire per legare il suo atteggiamento intellettuale a quello del maestro Salvemini e, conseguentemente, a quello del Cattaneo. Ritorna inoltre quell'immagine cara a Berneri del rapporto complesso tra idea e fatto, della «stella polare dell'Idea» che guida un cammino che però deve (e non può che) svolgersi sulle «vie della storia» affinché il pensiero possa giungere a realizzarsi politicamente. Nel corso del testo il lodigiano non perde inoltre l'occasione di ricordare che Salvemini «ha compilato una buona antologia del Cattaneo con un'introduzione che è un vero gioiello» ${ }^{599}$. Dopo un excursus biografico, Berneri bipartisce due periodi principali nello sviluppo del pensiero politico cattaneano, quello precedente al 1848 e quello successivo, caratterizzati rispettivamente dal tentativo di proporre riforme in senso federalista all'interno dell'Impero austriaco e dalla partecipazione al movimento di unità nazionale su posizioni repubblicane e anti-sabaude.

Berneri spiega che

\footnotetext{
${ }^{597}$ C. Berneri, Carlo Cattaneo, federalista, «Studi Sociali», VII, 4 maggio, 15 agosto e 20 novembre 1936, riprodotto in Id., Il federalismo libertario cit., pp. 92-109, e in Id., Anarchia e società aperta cit., pp. 324-337.

${ }^{598}$ Ibid.

${ }^{599} \mathrm{C}$. Berneri, Carlo Cattaneo, federalista cit. Il riferimento è, ovviamente, a Le più belle pagine di Carlo Cattaneo, scelte da Gaetano Salvemini, Fratelli Treves, Milano, 1922.
} 
il Cattaneo era ostilissimo al regime dispotico e centralista dell'Austria, disprezzava i patrizi collaboratori ed era irritato dallo spadroneggiare del clero. Ma pensava che male non minore del dominio austriaco sarebbe stato quello piemontese, essendo quella monarchia dispotica ed essendo in Piemonte ancor più dominante il clero, più gravi i privilegi feudali, assai meno liberi e più burocratizzati gli ordinamenti amministrativi ${ }^{600}$.

Nel saggio, come si sarà notato, avviene qualcosa di molto simile a quanto già avvenuto in quello su Kropotkin: da qualunque angolazione storica si scelga di vedere l'evoluzione del pensiero del repubblicano lombardo, la radice comune resta - nella ricostruzione berneriana - la lotta all'accentramento e allo sviluppo della burocrazia, prime cause della nascita del dispotismo e del privilegio; non stupisce che l'anarchico lodigiano sottolinei questo aspetto, che è funzionale ad avvicinare l'opera e il pensiero cattaneano all'anarchismo e, in ultima istanza, alla propria originale elaborazione di quest'ultimo. Mentre ripercorre tutta la vicenda biografica e politica del Cattaneo, Berneri torna spesso a rimarcare come il egli vedesse nel centralismo

la causa della pletora burocratica e del costituirsi della burocrazia in casta dominante. E insisteva nel dimostrare che il parlamento unico non può avere né il tempo né la competenza necessaria per risolvere i tanti e complessi problemi amministrativi, economici, giuridici, ecc., i quali variano profondamente dall'una all'altra regione. [...]

Nel sistema accentratore un'enorme massa di affari è sottratta alle competenze dei consigli locali e rovesciata a Roma, sì che il paese è schiavo della burocrazia e dei ministeri. Il governo federale, invece, affida agli uffici centrali le sole funzioni politiche di interesse nazionale, lasciando alle amministrazioni locali, più vicine agli interessi, tutta la direzione della vita locale ${ }^{601}$.

E Berneri non manca neanche di rilevare come il federalismo cattaneano abbia una forte e inequivocabile base comunalista:

Le regioni, i Comuni: ecco le basi del sistema federativo del Cattaneo. Le città sono per lui, come illustrava nel 1836, le "patrie locali", e "chi prescinde da questo amore delle patrie locali, seminerà sempre nella rena" ${ }^{602}$.

È nella conclusione del saggio berneriano che però emergono gli elementi più interessanti, in particolare il suo tornare a indirizzarsi ai repubblicani - e non è improbabile pensare che si stia rivolgendo in particolare al movimento "Azione Repubblicana Socialista», con cui riteneva possibile un'intesa - esortandoli affinché sviluppino un proprio programma ripensando alla propria tradizione politica e al contributo che il Cattaneo ha offerto:

${ }^{600}$ C. Berneri, Carlo Cattaneo, federalista, «Studi Sociali», VII, 15 agosto 1936.

${ }^{601}$ Id., Carlo Cattaneo, federalista, «Studi Sociali», VII, 20 novembre 1936.

${ }^{602}$ Ibid. 
A diffondere e ad elaborare il pensiero federalista del Cattaneo ha particolarmente contribuito La Critica Politica di O. Zuccarini, e anche non pochi giornali repubblicani, primo La Riscossa di Treviso. Ciò nonostante Cattaneo non è ancora abbastanza ben conosciuto dai repubblicani, ed è in considerazione di questa lacuna che ho scritto queste pagine [...]. Non mi è possibile dilungarmi ad esporre quei ritocchi e quegli sviluppi che la nostra concezione politica e sociale conduce ad apportare al sistema federalista del Cattaneo. Quanto tale sistema risponda ai tempi nostri, se contenga contraddizioni, se abbia costituzionali deficienze, potrà essere materia di discussione. Ma, per conto mio, anche gli anarchici hanno da guadagnare, per la loro cultura politica non solo, ma anche per una chiara ed organica visione dell'Italia rinnovata dalla rivoluzione antifascista e socialista, dalla conoscenza dell'opera di questo sommo scrittore ${ }^{603}$.

Da notare come Berneri torni a citare Zuccarini, promotore attraverso «La Critica Politica» di un intenso risvegliarsi delle idee federaliste all'interno del fronte repubblicano e di una vigorosa campagna di studio, approfondimento ed educazione politica che l'anarchico lodigiano aveva a suo tempo apprezzato e seguito con interesse. Nonostante le difficoltà nel riuscire a riunire un'insieme di partiti e movimenti intorno a un programma libertario, federalista e socialista, l'articolo berneriano apre ancora al confronto e alla discussione, sperando di riuscire a far avvicinare e integrare tra loro due tradizioni politiche che tanto potrebbero contribuire a indirizzare le sorti di una rivoluzione antifascista.

Il pensiero di Cattaneo - letto attraverso la lente di Salvemini e reinterpretato in chiave concretista rivoluzionaria - è stato spesso proposto da parte di Berneri ai suoi compagni come prossimo e compatibile con le idee libertarie e, come abbiamo visto, la stessa Costituzione ne conserva una traccia inconfondibile. Il federalismo del repubblicano lombardo potrebbe essere un terreno di incontro ideale, passibile ovviamente di ulteriori sviluppi. Come afferma Berneri nel suo saggio:

Se il suo federalismo non tiene conto, e non lo può, delle nuove forze direttive sorte e potenziate dalla grande industria, dalla vita sindacale, dal cooperativismo, è compito dei repubblicani d'avanguardia andare oltre il Cattaneo, sì che [...] la sua opera acquisti una funzione rivoluzionaria e ricostruttrice.

Cattaneo può ancora contribuire a fare della storia. Egli, che diceva che per navigare non ci vuol solo lume di stelle ma anche forza di venti, sarebbe ben lieto nel vedere l'Italia condotta dal grande vento della rivoluzione sociale più in là di quei limiti della sua prudenza positivista. E sarebbe ben lieto di vedere i repubblicani affrettarsi ad integrare e a dare più ampio respiro al proprio pensiero politico e sociale, alla vigilia, che dobbiamo volere prossima, di un nuovo '48, senza tradimenti di moderati ed illusioni di temporeggiamenti ${ }^{604}$.

Leggere questa conclusione e le piccole integrazioni di cui, a parere di Berneri, il federalismo cattaneano avrebbe bisogno, non può non farci tornare in mente la sua ${ }^{603}$ Ibid. I grassetti sono originali.

${ }^{604} \mathrm{C}$. Berneri, Carlo Cattaneo, federalista cit. Il grassetto è originale. 
idea per cui il sovietismo, libertariamente inteso, ne sarebbe stato il completamento ideale. Non può non farci tornare in mente le sue idee sulle autonomie degli organi sociali (consigli di fabbrica, sindacati, cooperative, consorzi, etc.) che avrebbero dovuto costituire, accanto ai Comuni, l'ossatura di un nuovo federalismo, che avrebbero dovuto - complice anche il successo dell'azione rivoluzionaria antifascista - essere capaci di sottrarre un elevato numero di compiti e attribuzioni agli organi centrali e di poter un domani renderli superflui, di sostituendoli in tutto e per tutto.

Il sovietismo e la riflessione sulle sue potenzialità sono in effetti tenuti ancora vivi da Berneri come argomenti di studio e di approfondimento. Nel luglio 1936, scrivendo a Carlo Frigerio, egli dichiara di essere al lavoro sull'opuscolo

«Stato e Rivoluzione», al quale vorrei far seguire «Bolscevismo e Sovietismo»; «Sovietismo e Anarchismo»; il «Comune libertario» ecc. ${ }^{605}$

Si può intuire che questa serie di opuscoli, purtroppo mai scritti, avrebbe potuto eventualmente costituire il nucleo centrale di un'opera di sintesi del pensiero berneriano sull'integrazione tra federalismo libertario, comunalismo e sovietismo. Tra le carte dell'Archivio Famiglia Berneri-Aurelio Chessa è conservato un breve manoscritto ancora inedito e privo di datazione, una "Introduzione" schematica e abbozzata, ci sembrerebbe essere stata pensata proprio per lo studio dedicato al rapporto tra sovietismo e anarchismo. Una nota aggiunta postuma sul documento sembrerebbe confermare la possibilità che l'introduzione sia stata pensata per il progettato opuscolo «Sovietismo e Anarchismo», collocandola dunque nel 1936; tale datazione è stata sostenuta anche da De Maria ${ }^{607}$. Berneri aveva sviluppato la seguente riflessione:

L'organizzazione degl'interessi collettivi richiede un sistema politico. Si presentano quattro sistemi possibili

$1^{\circ}$ L'amministrazione diretta, sistema nel quale il popolo in massa delibera volta per volta sulle varie questioni d'interesse generale, e provvede all'esecuzione delle sue deliberazioni;

$2^{\circ}$ La rappresentanza generica o autoritaria, sistema nel quale il popolo delega la propria sovranità ad un certo numero di persone da lui scelte e lascia a quelle il potere deliberativo ed esecutivo;

$3^{\circ}$ La democrazia propriamente detta, sistema nel quale il popolo delega le varie faccende d'interesse generale a dei tecnici, riservandosi di approvarne gli atti, controllando il loro operato, riservandosi di destituirli, ecc;

$4^{\circ}$ il sovietismo integrale, sistema nel quale l'amministrazione diretta e la democrazia si integrano, distaccandosi nettamente dalla rappresentanza autoritaria.

${ }^{605}$ Lettera di Camillo Berneri a Carlo Frigerio (Parigi, 6 luglio 1936), in Camillo Berneri, Epistolario inedito, vol. II cit. p. 150.

${ }^{606}$ Camillo Berneri, "Introduzione”, in AFB, fondo C. Berneri, cass. VII, Opere di carattere politico, $\mathrm{n}^{\circ} 5$, Russia.

${ }^{607}$ Vedi Carlo De Maria, Camillo Berneri cit., p. 161. 
Il regime bolscevico vigente nell'U.R.S.S. e la concezione bolscevica del sovietismo costituiscono una negazione dell'amministrazione diretta ed una deviazione della democrazia verso la rappresentanza autoritaria ${ }^{608}$.

Sono appunti particolarmente originali e in cui, notiamo, non si parla mai di anarchia o di "Stato libertario". Cattura immediatamente l'attenzione l'idea che la «democrazia propriamente detta» coincida con un sistema di deleghe tecniche controllabili e destituibili dal popolo, idea in effetti non nuova per Berneri e variamente declinata a partire dal 1919 con l'articolo L'autodemocrazia. Ora, secondo l'anarchico lodigiano, laddove questo sistema si integrasse con l'«amministrazione diretta», si avrebbe «sovietismo integrale», cosa che allontanerebbe così l'associazione mentale tra soviet e dittatura del proletariato: non a caso Berneri sottolinea immediatamente come l'Unione Sovietica abbia operato una deviazione rispetto alla concezione originaria del soviet, tipica argomentazione dalla quale si è sempre dovuto difendere ogni volta che provava a indicare nel sovietismo una strada da valutare per instaurare un'organizzazione di tipo libertario $^{609}$.

Gli appunti sono completati da alcune annotazioni sulle esperienze sovietiche della Rivoluzione russa, ma anche di quella ungherese e bavarese, esperienze sempre additate con interesse dall'anarchico lodigiano e che certo avrebbero dovuto costituire materia di studio e di approfondimento. Non è difficile comunque leggere in questo «sovietismo integrale» un rimando a quello che abbiamo oramai potuto fissare come uno dei perni del "programma minimo" berneriano, l'autonomismo degli organi sociali. I soviet, o consigli, avrebbero dovuto essere parte integrante di un progetto federalista - una sorta di "Repubblica dei consigli" libertaria - di cui Berneri, insieme ad alcuni compagni, aveva tentato di sbozzare la linee fondamentali nella Costituzione; un cantiere, come si può vedere, ancora aperto ed elemento portante del programma della lotta antifascista e della rivoluzione sociale da portare in Italia, con l'idea di emarginare nuove soluzioni di «rappresentanza generica o autoritaria».

Non sarebbe stata certo l'anarchia, ma almeno le fondamenta - costruite con le famose pietre offerte dall'epoca - su cui provare a edificarla.

\section{La Spagna, il banco di prova}

Sul finire del luglio 1936 Berneri ha abbandonato tutti i cantieri di studio ed elaborazione faticosamente aperti nel tempo per raggiungere Barcellona e andare a misurarsi direttamente con i fatti: in Spagna la resistenza repubblicana al tentativo di colpo di Stato da parte dei falangisti guidati da Franco (18-19 luglio 1936) ha aperto la strada a una vasta rivoluzione sociale in cui le organizzazioni libertarie della CNT-FAI stanno giocando un ruolo di primo piano, imprimendo agli eventi suggestivi sviluppi in senso anarchico. Barcellona e la regione della Catalogna sono in questo senso le roccaforti degli anarchici spagnoli, che si trovano a gestire quasi

${ }^{608}$ Camillo Berneri, "Introduzione" cit. Le sottolineature sono originali.

${ }^{609}$ Vedi supra. 
tutto in una volta non solo la resistenza militare, ma anche l'organizzazione politica ed economica ${ }^{610}$; ci sono, insomma, tutte le premesse per mettere in pratica le idee del collettivismo e del comunalismo libertario.

L'urgenza dell'azione porta Berneri a lavorare per l'organizzazione di una sezione italiana inquadrata all'interno della «Colonna Ascaso» della CNT-FAI, tuttavia il lodigiano non si dimostrerà particolarmente adatto e capace sul fronte e pertanto tornerà a Barcellona per occuparsi di incarichi politici. In ottobre il lodigiano avvia la pubblicazione del periodico "Guerra di Classe», che faceva eco col suo titolo alla storica testata dell'Unione Sindacale Italiana di tendenza anarcosindacalista: è l'inizio di una fase particolarmente intensa, in cui tutte le energie intellettuali e fisiche di Berneri si concentrano sull'analisi della situazione rivoluzionaria spagnola e dello scenario politico internazionale. Non è più il momento dello studio del programma, è il momento della realizzazione dello stesso ed è proprio in Spagna anzi che forse si potranno collaudare spunti, progetti e intuizioni da portare un domani in Italia.

Nonostante il frangente spagnolo non sia denso di contenuti o di sviluppi della riflessione federalista libertaria del Berneri, c'è un manoscritto ancora inedito di questo periodo che si ricollega alle elaborazioni politiche dell'anarchico lodigiano; il documento, dal titolo "Un esperimento cooperativista" 611 , non reca datazione, ma riteniamo possibile collocarlo nella fase finale del 1936, tra settembre e dicembre, quando Berneri ha oramai abbandonato il fronte e si è sovente recato in Francia per mantenere i contatti con i gruppi antifascisti. Il manoscritto sembra tornare sui temi dell'autonomismo che abbiamo visto essere così importante per l'anarchico lodigiano nell'elaborazione di un federalismo più dinamico, capace di integrare la dimensione economica e sociale a quella politica e territoriale. Curiosamente però non si parla di consigli o di soviet, bensì di un esperimento di cooperativa edile sperimentato in terra francese. "Cooperativa" è però termine insolito, non tra i più usati da Berneri; proprio per questo probabilmente egli decide di aprire il suo scritto con una breve storia del cooperativismo entro il movimento anarchico:

Il cooperativismo ha avuto, tra gli anarchici, varia e mutevole fortuna. Alle origini [...] gli anarchici furono agli avamposti, contribuendo a creare quelle oasi comuniste che furono le colonie libertarie e partecipando al movimento cooperativista socialista. Poi $[\ldots]$, fallita l'esperienza delle colonie libertarie, cominciò il distacco dell'anarco-comunismo dal movimento cooperativista socialista, e quel distacco andò sempre più accentuandosi con le deformazioni piccolo-borghesi di quel

\footnotetext{
${ }^{610}$ Per una ricostruzione degli eventi spagnoli si vedano almeno G. Ranzato, L'eclissi della democrazia: la guerra civile spagnola e le sue origini (1931-1939), Bollati Boringhieri, Torino, 2003; G. Jackson, La repubblica spagnola e la guerra civile (1931-1939), Il Saggiatore, Milano, 2003, e l'ancora indispensabile H. Thomas, Storia della guerra civile spagnola, Einaudi, Torino, 1963. Sul ruolo degli anarchici cfr. soprattutto C. Venza, Anarchia e potere nella guerra civile spagnola (1936-1939), Elèuthera, Milano, 2009.

${ }^{611} \mathrm{C}$. Berneri, "Un esperimento cooperativista", in AFB, fondo C. Berneri, cass. IV, Opere di carattere politico, $\mathrm{n}^{\circ} 3$, Rivoluzione e guerra di Spagna.
} 
movimento, irretito nei compromessi parlamentari ai quali condusse il protezionismo governativo $^{612}$.

Gli anarchici dunque dovrebbero essere recisi avversari delle cooperative, specialmente nella loro declinazione socialista riformista, per via del loro essere compromesse con il governo e la borghesia, per aver sviato i loro iscritti dalla lotta di classe per l'emancipazione sociale e politica. Ancora una volta però - come era stato per l'idea del soviet o per quella della Costituente - tutto dipende da come il termine e l'esperienza vengono interpretati:

Ma l'azione cooperativista è quello che si vuole che sia, e le deviazioni, le degenerazioni, le lacune non debbono far ignorare le possibilità feconde di esperimenti basati su di una chiara coscienza dei fini, dei mezzi e dei limiti. Incoerenze vi sono, prima quella di lavorare a contratto, sistema costituente una forma indiretta di lavoro a cottimo. Ma queste incoerenze permettono di sfuggire ad altre non minori: prima quella di rinunciare ad una notevole emancipazione economica sottoponendosi al comune sfruttamento dei salariati. Emancipazione economica che non ha soltanto un valore individuale, ma anche, e moralmente più, il significato, la missione sociale di un esempio ${ }^{613}$.

Da quelle che sono le possibili e interessanti opportunità che una cooperativa può idealmente garantire, Berneri passa a esporre il caso concreto che ha potuto osservare:

Di contro alle incoerenze si ergono, evidenti di significazione morale e fecondi di emancipazione sociale, i principi che presiedono alla creazione di una delle varie cooperative edili sorte in Francia e le norme che ne assicurano il funzionamento. Questa cooperativa è sorta con il compito precipuo di aiutare il movimento anarchico, sia finanziandone le iniziative (stampa all'estero e in Italia, comitati di soccorso, ecc.) sia sottraendo il maggior numero di compagni alle difficoltà di una situazione anormale, per la crisi di lavoro e per la difficoltà di regolarizzare la residenza. La cooperativa assicura ad alcuni dei suoi aderenti un guadagno che sarebbe loro difficile, e per taluni, impossibile trovare altrove, ma vi sono in essa aderenti che volontariamente rinunciano ad un guadagno superiore per lavorare associati, a favore dell'associazione ${ }^{614}$.

La cooperativa ha inoltre un'altra risorsa, quella di poter sperimentare il lavoro in comune anche con individualità non anarchiche e favorendo quindi un esempio concreto di collaborazione e solidarietà:

Le discussioni, la comunanza di fatiche, di sofferenze e di pericoli del lavoro, l'amicizia personale, lo scambio di pubblicazioni: tutto questo amalgama i simpatizzanti e i militanti, i comunisti e i socialisti, e gli uni e gli altri e noi.

${ }^{612} \mathrm{C}$. Berneri, "Un esperimento cooperativista" cit., c. 1.

${ }^{613}$ Ivi, c. 2.

${ }^{614}$ Ivi, c. 3 . 
Purtroppo non sempre vi è in tutti gli anarchici aderenti quella tolleranza verso le opposte idee, che è condizione prima per un'esatta comprensione e un'abile propaganda spicciola, ma alle intolleranze di taluni supplisce e compensa la mitezza di altri, sì che vi è, nel complesso, un'atmosfera di cordialità e di intesa che è tanto più efficace oggi che $\mathrm{i}$ dissidi tra $\mathrm{i}$ partiti di avanguardia sono approfonditi $\mathrm{e}$ febbrili ${ }^{615}$.

Riuscire a far penetrare idee e principi libertari negli avversari politici è senz'altro positivo per Berneri, è l'inizio indispensabile per costruire l'argine al prevalere di soluzioni autoritarie e stataliste; l'esperimento dimostra insomma che «la rivoluzione delle cose non basta, e che occorre una ben vasta e profonda evoluzione degli spiriti per realizzare il comunismo libertario» ${ }^{616}$. L'anarchico lodigiano è pertanto soddisfatto dell'iniziale successo della cooperativa e ritiene che essa sia da considerare un mezzo, sempre tenendo presente però che bisogna fuggire «l'illusoria credenza nella possibilità di combattere efficacemente il dominio capitalistico opponendogli la concorrenza di una rete di cooperative di produzione e di consumo»> ${ }^{617}$.

La cooperativa da sola in altre parole non basta e non ci si può accontentare di ritagliare un'oasi di produzione e consumo all'interno di un sistema economico, sociale e politico che si intende demolire per ricostruire una nuova organizzazione su basi diverse, libertarie, federaliste e autonomiste. La cooperativa, in altre parole, è l'ennesimo nucleo in cui prepararsi per la rivoluzione:

Anarchici, tendenzialmente comunisti, vediamo nel cooperativismo una forma di mutuo appoggio ispirata a generose intenzioni di assistenza e di lotta, e un'esperienza preparatrice a quella diretta ed egualitaria gestione della vita economica del paese, che conquisteremo con la forza armata, con la espropriazione del latifondo e dell'officina, con la confisca dei capitali bancari, e che costruiremo mediante una rete di consigli di operai e di contadini, di liberi comuni, di consigli provinciali e regionali, di congressi nazionali costituiti da rappresentanze tecniche, di direzioni generali aventi un carattere strettamente esecutivo. La pratica cooperativistica deve creare dei nuclei di operai capaci di direzione tecnica ed amministrativa, deve creare l'abitudine e la capacità ${ }^{618}$.

Le coordinate, come si può vedere, sono rimaste le stesse del "programma minimo" concretizzatosi nella Costituzione, nell'idea di una «Federazione Italiana Comuni Socialisti» di tipo ascendente, in cui gli organi governativi vengono sostituiti da quelli amministrativi; di nuovo, la "Repubblica dei consigli" libertaria. Bisogna essere però preparati, pronti a offrire le famose soluzioni concrete ai problemi che la rivoluzione porrà e che già in quel momento sta ponendo in Spagna; ancora una volta, quel concretismo rivoluzionario e quell'impostazione

${ }^{615} \mathrm{C}$. Berneri, "Un esperimento cooperativista” cit., c. 3

${ }^{616}$ Ibid.

${ }^{617}$ Ivi, c. 5 .

${ }^{618} \mathrm{C}$. Berneri, "Un esperimento cooperativista" cit., c. 5. I corsivi sono nostri. 
problemistica derivati dalla lezione di Salvemini che continua a riecheggiare nelle parole dell'anarchico lodigiano.

Berneri ha serbato fino in fondo le idee che ha sviluppato, anche quando volge lo sguardo verso l'avvenire e pensa all'insurrezione antifascista come rivoluzione sociale libertaria:

Domani, rientrando in Italia, vi porteremo un'esperienza in più, e sarà tanto meno possibile il dominio dei demagoghi e la formazione di una nuova borghesia burocratica e tecnica quanto più in ogni città, in ogni villaggio, in ogni campagna esisteranno gruppi di comunisti capaci di erigere un'ordine nuovo e migliore ${ }^{619}$.

Tutto dipenderà, in ultima istanza, dagli anarchici stessi: quanto più sapranno incidere politicamente, tanto più indirizzeranno la rivoluzione; quanto più mostreranno di avere una serie di proposte concrete e tecnicamente applicabili, tanto più potranno evitare il rafforzarsi di soluzioni centraliste e autoritarie; quanto più vorranno e si prepareranno, tanto più potranno. Certo Berneri aveva intenzione di percorrere quella strada fino in fondo e avrebbe cercato di portare con sé quanti più compagni possibile, tentando altresì di favorire una convergenza di altre formazioni politiche su una piattaforma programmatica di tipo federalista, libertario e socialista.

Così, significativamente, si conclude la sua elaborazione politica su questo asse portante: l'anarchico lodigiano aveva in effetti messo in cantiere l'ennesimo progetto di libro, questa volta sul tema del municipalismo; avrebbe dovuto intitolarsi "La nuova organizzazione municipale in Spagna", ma la sua stesura non inizierà mai e tutto ciò che oggi ci resta è soltanto una serie di materiali (ritagli di giornale, opuscoli, estratti da libri, etc.) raccolti tra il 1936 e il 1937 e conservati presso l'Archivio Famiglia Berneri-Aurelio Chessa ${ }^{620}$. L'esperienza spagnola forse avrebbe offerto a Berneri nuove e numerose suggestioni, probabilmente lo avrebbe portato a sviluppare ulteriormente le sue idee federaliste e comunaliste libertarie alla luce di quello che è stato con ogni probabilità il frangente rivoluzionario in cui il movimento anarchico è riuscito a tradurre maggiormente in pratica i propri postulati cardinali.

Restano tuttavia solo delle ipotesi e delle supposizioni: il suo arresto e la sua uccisione a Barcellona nella notte tra il 4 e il 5 maggio 1937 hanno tragicamente e prematuramente posto fine ai suoi pensieri e alle sue battaglie.

\footnotetext{
${ }^{619}$ Ibid.

${ }^{620}$ Raccolta di materiale documentario sul tema "Municipio", per l'elaborazione di un'opera mai scritta "La nuova organizzazione municipale in Spagna", in AFB, fondo C. Berneri, cass. IV, Opere di carattere politico, $\mathrm{n}^{\circ} 3$ cit.
} 


\section{Bibliografia}

\section{Fonti su Camillo Berneri citate o consultate}

Archivio Centrale dello Stato, Ministero dell'Interno, Casellario Politico Centrale, Berneri Camillo, busta 537, fascicolo I, II e III

Archivio Storico dell'Università degli Studi di Firenze, Regio Istituto di Studi

Superiori, Pratici e di Perfezionamento in Firenze. Sezione di Filosofia e Filologia. Registro della carriera scolastica degli studenti X (1914-1917)

Archivio Famiglia Berneri-Aurelio Chessa, fondo C. Berneri, cassetta I, ${ }^{\circ} 1$, Carte personali

Archivio Famiglia Berneri-Aurelio Chessa, fondo C. Berneri, cassetta IV, $\mathrm{n}^{\circ}$ 1, Anarchia e anarchismo

Archivio Famiglia Berneri-Aurelio Chessa, fondo C. Berneri, cassetta IV, $\mathrm{n}^{\circ}$

3, Rivoluzione e guerra di Spagna

Archivio Famiglia Berneri-Aurelio Chessa, fondo C. Berneri, cassetta VII, $\mathrm{n}^{\circ}$ 5, Russia

Archivio Famiglia Berneri-Aurelio Chessa, fondo C. Berneri, cassetta XI, $n^{\circ}$ 2, Scritti di critica storica, letteraria, filosofica

Archivio Famiglia Berneri-Aurelio Chessa, conservato separatamente, "Costituzione della Federazione Italiana Comuni Socialisti - (F.I.C.S.)"

\section{Scritti editi di Camillo Berneri citati o consultati}

Berneri Camillo, Il pensiero sociale di Alfred Russel Wallace, «L'Avanguardia», VIII, 8 marzo 1914

B. C., La proprietà è un furto, «L'Avanguardia», VIII, 19 luglio 1914

— , Da dove la Chiesa ha rubato i suoi culti?, «L'Avanguardia», VIII, 26 luglio 1914

Berneri Camillo, Effetti di guerra in un paese di pace, «La Folla», III, 16 agosto 1914 
—, Un pittore e un poeta pacifisti: Rubens e Fagiuoli, «L'Avanguardia», VIII, 1 novembre 1914

—, Agli anglofili, «L'Avanguardia», VIII, 8 novembre 1914

- , Confessioni di un anglofobo, «L'Avanguardia», VIII, 6 dicembre 1914

B. C., Demetrio Tutzewitsch, «L'Avanguardia», VIII, 13 dicembre 1914

Berneri Camillo, Augusto Blanqui, «L'Avanguardia», VIII, 20 dicembre 1914

B. C., Uomini e idee. Paola Mink, «L'Avanguardia», IX, 10 gennaio 1915

Berneri Camillo, Riabilitazioni guerraiole, «L'Avanguardia», IX, 14 febbraio 1915

—, Dopo i fatti di Reggio Emilia, «L'Avanguardia», IX, 14 marzo 1915

— , Piaghe di casa nostra, «L'Avanguardia», IX, 4 aprile 1915

— , La pittura sociale. Karel Myelbeck di Praga, «L'Avanguardia», IX, 4 luglio 1915

—, La guerra e la pace nell'arte, «L'Avanguardia», IX, 25 luglio 1915

B. C., Perché $i$ sovrani si chiamano...come si chiamano, «L'Avanguardia», $\mathrm{X}, 21$ maggio 1916

— , La confessione, «L'Avanguardia», X, 4 giugno 1916

—, Pangermanesimo in crisi, «L'Avanguardia», X, 2 luglio 1916

Berneri Camillo, Lettera aperta ai Giovani socialisti, «L'Avvenire Anarchico», IV, 28 luglio e 4 agosto 1916

B. C., Maternità militarizzata, «L'Avanguardia», X, 13 agosto 1916

Berneri Camillo, Novella storica. L'Ercole di Valle d'Illiez, «L'Avanguardia», X, 29 ottobre 1916

_ , Per un silenzio ingiusto, «Guerra di Classe», III, 22 aprile 1917

—, Mazzini e Bismarck, «L'Avvenire Anarchico», VIII, 29 giugno 1917

— , Mentre i giovani socialisti polemizzano, «Guerra di Classe», III, 30 giugno 1917

B. C., Tradeunionismo e sindacalismo in Inghilterra, «Guerra di Classe», III, 11 agosto 1917

Berneri Camillo, I mazziniani e la guerra monarchico-repubblicana, «L'Avvenire Anarchico», VIII, 17 agosto 1917

—, Con Kerenskij o con Lenin, «Guerra di Classe», III, 6 ottobre 1917

— , Da Kipling a Chamberlain, «L'Avvenire Anarchico», VIII, 12 e del 26 ottobre 1917

— , Fuori dal partito socialista non v'è azione socialista?, «L'Avvenire Anarchico», VIII, 26 ottobre 1917

— , Un pedagogista moderno. Jean Lightart, «L'Università Popolare», XVII, 15 dicembre 1917 
B. C., Asterischi. Della filosofia del dopoguerra, «Il Grido», II, 20 settembre 1918

Berneri Camillo, Le marionette storiche, «Il Grido», II, 5 novembre 1918

Camillo da Lodi, I problemi dell'unità rivoluzionaria, «Il Libertario», XVII, 1 maggio 1919

— , Il partito repubblicano di fronte alla rivoluzione, «Volontà», I, 1 maggio 1919

—, I limiti dell'unità rivoluzionaria, «Il Libertario», XVII, 15 maggio 1919

—, L'autodemocrazia, «Volontà», I, 1 giugno 1919

- , I doveri di una conquista classista. Le 8 ore di lavoro e la cultura popolare, «Volontà», I, 16 settembre 1919

- , I problemi della Rivoluzione. I rapporti fra la Città e le Campagne, «Volontà», I, 1 dicembre 1919

— , I problemi della rivoluzione. Parentesi, «Volontà», II, 16 gennaio 1920

—, Il momento attuale e l'unità rivoluzionaria, «Umanità Nova», I, 9 marzo 1920

_, Pane e giustizia per le Puglie!, «Umanità Nova», I, 8 ottobre 1920

C. B., La politica della volpe di Dronero, «Umanità Nova», I, 26 ottobre 1920

— , La politica del ministro della malavita, «Umanità Nova», I, 27 ottobre 1920

—, La Dalmazia italiana?, «Umanità Nova», I, 17 dicembre 1920

—, Stato e burocrazia, «Umanità Nova», I, 25 dicembre 1920

— , La crisi dello Stato, «Umanità Nova», II, 3 settembre 1921

- , Stato e Comune secondo Giuseppe Mazzini, «Umanità Nova», II, 14 settembre 1921

— , Gli equivoci del parlamentarismo, «Umanità Nova», II, 19 novembre 1921

Berneri Camillo, Decentramento e conservazione statale, «Umanità Nova», II, 26 novembre 1921

—, Stato e burocrazia in Russia, «Umanità Nova», II, 29 dicembre 1921

- , Repubblicanesimo sociale e anarchismo. In margine alla polemica Ansaldi-Malatesta, «Umanità Nova», III, 16 aprile 1922, segue nota di Errico Malatesta

—, A proposito di libertà, «Umanità Nova», III, 23 settembre 1922

- , Anarchismo e federalismo - Il pensiero di Camillo Berneri, «Pagine Libertarie», II, 20 novembre 1922

_ , Il liberalismo nell'Internazionale, «Rivoluzione Liberale», II, 24 aprile 1923

C. B., Della tolleranza, «Fede!», II, 20 aprile 1924 
Berneri Camillo, Libertà ed autorità, «Fede!», II, 22 giugno 1924

- , Risposta ad una consultazione sui compiti immediati e futuri dell'anarchismo, «La Revue Internationale Anarchiste», I, 15 gennaio 1925

—, Un federalista russo. Pietro Kropotkin, Edizioni Fede!, Roma, 1925

— , Prefazione a Pietro Kropotkin [Pëtr Kropotkin], Il mutuo appoggio: un fattore dell'evoluzione, Casa Editrice Sociale, Milano, 1925

C. B., I libri, «Pensiero e Volontà», III, 1 marzo 1926

Berneri Camillo, L'uomo finito cattolico, «Fede!», IV, 21 aprile 1926

— , La Comune di Parigi e l'idea federalista, «Culmine», II, 27 aprile 1926

C. B., L'antifascismo in Francia, «Il Risveglio comunista-anarchico», XXVI, 5 marzo 1927

Berneri Camillo, Nord e Sud, «La Lotta Umana», I, 30 settembre 1928 e II, 20 ottobre 1928

C. B., La nostra ora, «Il Risveglio anarchico», XXVII, 18 maggio 1929

Berneri Camillo, Per le autonomie locali, «Vogliamo!», I, 1 agosto 1929

—, L'ora dell'anarco-sindacalismo, «Guerra di Classe», settembre 1930

—, Un aborto possibile, «L'Adunata dei Refrattari», IX, 6 settembre 1930

— , Il movimento "Giustizia e Libertà", «L'Adunata dei Refrattari», IX, 1 novembre 1930

—, Mali passi o fisime?, «Guerra di Classe», II, gennaio 1931

— , Fallimento o crisi?, «Guerra di Classe», II, gennaio 1931

C. B., La tattica fumogena, «L'Adunata dei Refrattari», XI, 10 gennaio 1932

Berneri Camillo, L'operaiolatria, «Guerra di Classe», III, 30 aprile 1932

- , Gli anarchici e $i$ comitati locali rivoluzionari, "L'Adunata dei Refrattari», XI, 18 giugno 1932, segue nota della redazione

—, Del diritto alla critica, «L'Adunata dei Refrattari», XI, 2 luglio 1932

L'Orso, La Costituente, «L'Adunata dei Refrattari», XI, 23 luglio 1932

Berneri Camillo, Il problema delle autonomie locali, «Guerra di Classe», III, agosto 1932

—, Il dilettantismo culturale, «L'Adunata dei Refrattari», XI, 6 agosto 1932

—, La pre-anarchia, «L'Adunata dei Refrattari», XI, 17 settembre 1932

—, Sovietismo, anarchismo e anarchia, «L'Adunata dei Refrattari», XI, 15 ottobre 1932, segue nota di m. s. [Max Sartin]

— , I repubblicani di destra, gli altri e noi, «Umanità Nova», I, 20 ottobre 1932

—, Soviettismo e bolscevismo, «Umanità Nova», I, 10 novembre 1932

— , L'operaiolatria, Gruppo d'Edizioni Libertarie, Brest, 1934

— , Gli anarchici e "G. e L.", "Giustizia e Libertà», II, 6 dicembre 1935, segue Risposta di Carlo Rosselli 
— , Discorso sul federalismo e l'autonomia, «Giustizia e Libertà», II, 27 dicembre 1935

— , Come vedo il movimento giellista, «L'Adunata dei Refrattari», XV, 4 aprile 1936, segue nota di M. S. [Max Sartin]

—, Carlo Cattaneo, federalista, «Studi Sociali», VII, 4 maggio, 15 agosto e 20 novembre 1936

\section{Raccolte e antologie di scritti di Camillo Berneri}

Berneri Camillo, Pensieri e battaglie, Comitato Camillo Berneri, Parigi, 1938

—, Pietrogrado 1917 Barcellona 1937. Scritti scelti, a cura di Pier Carlo Masini e Alberto Sorti, Sugar, Milano 1964

—, Epistolario inedito, vol. I, a cura di Aurelio Chessa e Pier Carlo Masini, Edizioni Archivio Famiglia Berneri, Pistoia, 1980

— , Epistolario inedito, vol. II, a cura di Luigi Di Lembo e Paola Feri, Edizioni Archivio Famiglia Berneri, Pistoia, 1984

— , Il federalismo libertario, a cura di Patrizio Mauti, La Fiaccola, Ragusa, 1992

- , Anarchia e società aperta. Scritti editi ed inediti, a cura di Pietro Adamo, M\&B, Milano, 2001

—, Scritti scelti, Zero in condotta, Milano, 2013

\section{Letteratura secondaria: opere, studi e scritti su Camillo Berneri}

Adamo Pietro, Introduzione a Camillo Berneri, Anarchia e società aperta. Scritti editi ed inediti, M\&B, Milano, 2001

-, Dai feudi di Camillo Prampolini: Camillo Berneri e la tradizione socialista, in Giorgio Boccolari, Luciano Casali (a cura di), Prampolini e il socialismo reggiano, «L'Almanacco», XX, n³7, dicembre 2001

Atti del Convegno di studi su Camillo Berneri, Milano, 9 ottobre 1977, La Cooperativa Tipografica Editrice, Carrara, 1979

Berti Giampietro, Berneri e Rosselli: anarchismo e socialismo liberale a confronto, in Michela Nacci (a cura di) Figure del liberalsocialismo, Centro Editoriale Toscano, Firenze, 2010

Berti Giampietro, Sacchetti, Giorgio (a cura di), Un libertario in Europa. Camillo Berneri: fra totalitarismi e democrazia. Atti del convegno di studi storici, Arezzo, 5 maggio 2007, Edizioni Biblioteca Panizzi e Archivio Famiglia Berneri - Aurelio Chessa, Reggio Emilia, 2010 
Camillo Berneri, singolare/plurale. Atti della giornata di studi, Reggio Emilia, 28 maggio 2005, Edizioni Biblioteca Panizzi e Archivio Famiglia Berneri-Aurelio Chessa, Reggio Emilia, 2007

Carrozza Gianni [Giovanbattista], Camillo Berneri ed il dibattito antimilitarista nella Federazione Giovanile Socialista, «Università di Firenze, Facoltà di Magistero - Annali dell'Istituto di Storia», III, 19821984, Leo S. Olschki, Firenze, 1985

- , Note per una bibliografia di Berneri, appendice a Camillo Berneri, Scritti scelti, Zero in condotta, Milano, 2013

Cerrito Gino, Introduzione a Camillo Berneri, Scritti scelti, Zero in condotta, Milano, 2013

D'Errico Stefano, Anarchismo e politica. Nel problemismo e nella critica dell'anarchismo del ventesimo secolo, il "programma minimo" dei libertari del terzo millennio. Rilettura antologica e biografica di Camillo Berneri, Mimesis, Milano, 2007

De Maria Carlo, Camillo Berneri. Tra anarchismo e liberalismo, Franco Angeli, Milano, 2004

- , Una famiglia anarchica. La vita dei Berneri tra affetti, impegno ed esilio nell'Europa del Novecento, Viella, Roma, 2019

Fochi Adalgisa, Con te, figlio mio!, Officina Grafica Franchising, Parma, 1948

Gervasoni Marco, Il filo rosso della "inappartenenza": Berneri e Tasca, «Rivista Storica dell'Anarchismo», IV, n 1 , gennaio-giugno 1997

Guidi Flavio, Nostra patria è il mondo intero! Camillo Berneri e «Guerra di Classe» a Barcellona (ottobre '36-novembre '37), edito a cura dell'autore, Brescia, 2010

Madrid Santos, Francisco, Camillo Berneri, un anarchico italiano (18971937). Rivoluzione e controrivoluzione in Europa (1917-1937), Edizioni Archivio Famiglia Berneri, Pistoia, 1985

Masini Pier Carlo, Camillo Berneri alla scuola di Prampolini, appendice a Camillo Berneri, Mussolini, psicologia di un dittatore, Edizioni Azione Comune, Milano, 1966

Memoria antologica, saggi critici e appunti biografici in ricordo di Camillo Berneri nel cinquantesimo della morte, Edizioni Archivio Famiglia Berneri, Pistoia, 1986

Montanari Fabrizio, La giovinezza di Berneri, «Ricerche Storiche», XXXI, $\mathrm{n}^{\circ} 83$, dicembre 1997

Pechar Saverio Werther, Il caso Berneri. Antifascisti italiani nella Spagna rivoluzionaria (1936-1937), Edizioni ANPPIA, Roma, 2017

Salvemini Gaetano, Donati e Berneri, «Il Mondo», IV, 3 maggio 1952 
Scavino Marco, Berneri, Gobetti e la rivoluzione italiana, «Rivista Storica dell'Anarchismo», IV, n 1 , gennaio-giugno 1997

Strambi Claudio, L'inquieta attitudine: Camillo Berneri e la vicenda politica dell'anarchismo in Italia. Primo Libretto, Edizioni Kronstadt, Pisa, 2015

- , L'inquieta attitudine: Camillo Berneri e la vicenda politica dell'anarchismo in Italia. Secondo Libro, "Il biennio rosso e rossonero", Edizioni Kronstadt, Pisa, 2017

Tasca Angelo, Camillo Berneri, «Il nuovo Avanti», IV, 22 maggio 1937

\section{Fonti archivistiche}

Archivio Centrale dello Stato, Ministero dell'Interno, Direzione Generale Pubblica Sicurezza, Divisione Polizia Politica, Fascicoli per materia, busta 25, fascicolo 1, Francia, anarchici italiani

Archivio Centrale dello Stato, Ministero dell'Interno, Direzione Generale Pubblica Sicurezza, Divisione Polizia Politica, Fascicoli per materia, busta 124, fascicolo 8, Rapporti fra Giustizia e Libertà e anarchici

Archivio Centrale dello Stato, Ministero dell'Interno, Direzione Generale Pubblica Sicurezza, Divisione Polizia Politica, Fascicoli per materia, busta 125, fascicolo 1, Giustizia e Libertà, Parigi (riunioni, aderenti)

Archivio Centrale dello Stato, Ministero dell'Interno, Direzione Generale Pubblica Sicurezza, Divisione Polizia Politica, Fascicoli per materia, busta 127, fascicolo 1c, Giustizia e Libertà, Parigi

Archivio Centrale dello Stato, Ministero dell'Interno, Direzione Generale Pubblica Sicurezza, Divisione Polizia Politica, Fascicoli per materia, busta 127, fascicolo 1d, Giustizia e Libertà, Parigi

Istituto Storico della Resistenza in Toscana, Archivio Gaetano Salvemini, sezione I, Manoscritti e materiali di lavoro, fascicolo I, Manoscritti e materiali di lavoro dal 1898 all'esilio, $\mathrm{n}^{\circ}$ 8, Appunti e materiali di studio per corsi di lezione e conferenze su Giuseppe Mazzini

Istituto Storico della Resistenza in Toscana, Archivio Gaetano Salvemini, sezione I, Manoscritti e materiali di lavoro, fascicolo I, Manoscritti e materiali di lavoro dal 1898 all'esilio, $\mathrm{n}^{\circ}$ 9, Materiali di lavoro e redazioni preparatorie per l'introduzione al libro Le più belle pagine di Carlo Cattaneo, scelte da Gaetano Salvemini

Istituto Storico della Resistenza in Toscana, Archivi di Giustizia e Libertà, fondo C. Rosselli, busta 1, sezione 1 


\section{Fonti bibliografiche}

Acciai Enrico, Antifascismo, volontariato e guerra civile in Spagna. La sezione italiana della Colonna Ascaso, UNICOPLI, Milano, 2016

Albanese Giulia, La Settimana Rossa tra aspirazioni rivoluzionarie e reazioni d'ordine, in Mario Isnenghi, Simon Levis Sullam (a cura di) Gli Italiani in guerra. Conflitti, identità, memorie dal Risorgimento ai nostri giorni, vol. II, Le «Tre Italie»: dalla presa di Roma alla Settimana Rossa (1870-1914), Utet, Torino, 2008

Ambrosoli Luigi, Né aderire né sabotare, 1915-1918, Edizioni Avanti!, Milano, 1961

Anarchici e anarchia nel mondo contemporaneo. Atti del Convegno promosso dalla Fondazione Luigi Einaudi (Torino, 5, 6, e 7 dicembre 1969), Fondazione Luigi Einaudi, Torino, 1970

Andreucci Franco, Detti Tommaso (a cura di), Il movimento operaio italiano. Dizionario biografico, 1853-1943, vol. II, Editori Riuniti, Roma, 1976

— , Il movimento operaio italiano. Dizionario biografico, 1853-1943, vol. III, Editori Riuniti, Roma, 1977

—, Il movimento operaio italiano. Dizionario biografico, 1853-1943, vol. V, Editori Riuniti, Roma, 1978

Angelini Giovanna, L'altro socialismo. L'eredità democraticorisorgimentale da Bignami a Rosselli, Franco Angeli, Milano, 1999

Antonioli Maurizio, Gli anarchici italiani e la prima guerra mondiale, «Rivista Storica dell'Anarchismo», II, n¹, gennaio-giugno 1995

Antonioli Maurizio, Masini Pier Carlo, Il sol dell'avvenire. L'anarchismo in Italia dalle origini alla prima guerra mondiale, Edizioni Biblioteca Franco Serantini, Pisa, 1999

Antonioli Maurizio et al. (diretto da), Dizionario Biografico degli anarchici italiani, vol. I, Edizioni Biblioteca Franco Serantini, Pisa, 2003

- , Dizionario Biografico degli anarchici italiani, vol. II, Edizioni Biblioteca Franco Serantini, Pisa, 2004

Arfé Gaetano, Storia del socialismo italiano (1892-1926), Einaudi, Torino, 1965

— , Il movimento giovanile socialista. Appunti sul primo periodo (19031912), Edizioni Del Gallo, Milano, 1973

Aruffo Alessandro, Breve storia degli anarchici italiani, 1870-1970, Datanews, Roma, 2006

Asor Rosa, Alberto, La cultura, in Storia d'Italia, vol. IV, Dall'Unità a oggi, t. 2, Einaudi, Torino, 1975

Bagnoli Paolo, Rosselli, Gobetti e la rivoluzione democratica. Uomini e idee tra liberalismo e socialismo, La Nuova Italia, Firenze, 1996 
Barbadoro Idomeneo, Biennio rosso: lotte sociali e direzione socialista, in Id. (coordinata da), Storia della società italiana, vol. XXI, Teti, Milano, 1982

Bennassar Bartolomé, La guerra di Spagna: una tragedia nazionale, Einaudi, Torino, 2006

Berti Giampietro, Il pensiero anarchico. Dal Settecento al Novecento, Lacaita, Manduria-Bari-Roma, 1998

— , Errico Malatesta e il movimento anarchico italiano e internazionale, Franco Angeli, Milano, 2003

Bertolucci Franco, A Oriente sorge il sol dell'avvenire. La rivoluzione russa vista dagli anarchici italiani 1917-1922, Edizioni Biblioteca Franco Serantini, Pisa, 2017

Bettini, Leonardo, Bibliografia dell'anarchismo, vol. I, t. 1, Periodici e numeri unici anarchici in lingua italiana pubblicati in Italia (18721971), Edizioni Crescita Politica, Firenze, 1972

- , Bibliografia dell'anarchismo, vol. I, t. 2, Periodici e numeri unici anarchici in lingua italiana pubblicati all'estero (1872-1971), Edizioni Crescita Politica, Firenze, 1976

Bianciardi Silvia, Camillo Prampolini, costruttore di socialismo, Il Mulino, Bologna, 2012

Boccolari Giorgio, Casali Luciano (a cura di), Prampolini e il socialismo reggiano, «L'Almanacco», XX, n³7, dicembre 2001

Bresciani Marco, Quale antifascismo? Storia di Giustizia e Libertà, Carocci, Roma, 2017

Campos Boralevi Lea (a cura di), Challengin centralism. Decentramento e autonomie nel pensiero politico europeo, Firenze University Press, Firenze, 2011

Capetta Francesca, Piccolo Sara (a cura di), Archivio storico dell'Università degli Studi di Firenze (1860-1960). Guida inventario, Firenze University Press, Firenze, 2004

Caretti, Stefano, Il socialismo italiano e la "grande guerra», in Stefano Caretti, Zeffiro Ciuffoletti e Maurizio Degl'Innocenti (a cura di), Lezioni di storia del Partito Socialista Italiano, 1892-1976, Cooperativa Editrice Universitaria, Firenze, 1977

Carlo e Nello Rosselli (1937-2017), «Rivista storica del socialismo», Nuova serie, II, $\mathrm{n}^{\circ} 1$, maggio 2017

Casali Luciano, Sovversivi e costruttori. Sul movimento operaio in EmiliaRomagna, in Roberto Finzi (a cura di), Storia d'Italia, Le regioni dall'Unità ad oggi. L'Emilia-Romagna, Einaudi, Torino, 1997 
Cecchini, Lucio, Unitari e federalisti. Il pensiero autonomistico repubblicano da Mazzini alla formazione del P.R.I., Bulzoni, Roma, 1974

Cerasi Laura, Gli ateniesi d'Italia: associazioni di cultura a Firenze nel primo Novecento, Franco Angeli, Milano, 2000

Cerrito Gino, L'antimilitarismo anarchico in Italia nel primo ventennio del secolo, Edizioni RL, Pistoia, 1968

—, Il ruolo della organizzazione anarchica: l'efficientismo organizzativo, il problema della minoranza, il periodo transitorio, classismo e umanesimo, Edizioni RL, Catania, 1973

- , Dall'insurrezionalismo alla settimana rossa: per una storia dell'anarchismo in Italia (1881-1914), Edizioni Crescita Politica, Firenze, 1977

- , L'emigrazione libertaria italiana in Francia nel ventennio fra le due guerre, in Bruno Bezza (a cura di), Gli italiani fuori d'Italia. Gli emigrati italiani nei movimenti operai dei paesi d'adozione, 1880-1940, Franco Angeli, Milano, 1983

Ceva Lucio, Spagne 1936-1939: politica e guerra civile, Franco Angeli, Milano, 2010

Chessa Aurelio (a cura di), Convegno d'intesa degli anarchici italiani emigrati in Europa (Francia - Belgio - Svizzera), Parigi/Ottobre 1935, Pistoia, Edizioni Archivio Famiglia Berneri, 1980

Chiaramonte Umberto, Il dibattito sulle autonomie nella Storia d'Italia (1796-1996). Unità-federalismo-regionalismo-decentramento, Franco Angeli, Milano, 1998

Chomsky, Noam, Anarchia e libertà. Scritti e interviste, Datanews, Roma, 2006

—, Il governo del futuro, Tropea, Milano, 2009

Cingari Gaetano (a cura di), Gaetano Salvemini tra politica e storia, Laterza, Roma-Bari, 1986

Ciuffoletti Zeffiro (a cura di), Nello Rosselli. Uno storico sotto il fascismo. Lettere e scritti vari (1924-1937), La Nuova Italia, Firenze, 1979

Ciuffoletti Zeffiro, Degl'Innocenti Maurizio, Sabbatucci Giovanni, Storia del PSI, vol. I, Le origini e l'età giolittiana, Laterza, Roma-Bari, 1992

Ciuffoletti Zeffiro, Federalismo e regionalismo. Da Cattaneo alla Lega, Laterza, Roma-Bari, 1994

Colombo Arturo (a cura di), I colori della libertà. Il mondo di Nello Rosselli fra storia, arte e politica, Franco Angeli, Milano, 2003

Cortesi Luigi, Il PSI e la Grande Guerra, «Rivista storica del socialismo», X, $\mathrm{n}^{\circ} 32,1967$ 
Dadà Adriana, Gli anarchici italiani fra guerra di classe e reazione, in Idomeneo Barbadoro (coordinata da), Storia della società italiana, vol. XXI, Teti, Milano, 1982

- , L'anarchismo in Italia: fra movimento e partito. Storia e documenti dell'anarchismo italiano, Teti, Milano, 1984

De Caro Gaspare, Gaetano Salvemini, Utet, Torino, 1970

De Marco Laura, Il soldato che disse no alla guerra: storia dell'anarchico Augusto Masetti (1888-1966), Spartaco, Santa Maria Capua Vetere, 2003

Degl'Innocenti Maurizio, Geografia e istituzioni del socialismo italiano, 1892-1914, Guida, Napoli, 1983

—, Gaetano Salvemini e le autonomie locali, Lacaita, Manduria-Bari-Roma, 2007

— , Camillo Prampolini e il socialismo del suo tempo, «Storia e Futuro», VIII, n¹9, febbraio 2009

—, La patria divisa. Socialismo, nazione e guerra mondiale, Franco Angeli, Milano, 2015

Della Peruta Franco, I democratici e la rivoluzione italiana, Feltrinelli, Milano, 1958

—, Democrazia e socialismo nel Risorgimento, Editori Riuniti, Roma, 1973

Di Lembo Luigi, Il federalismo libertario e anarchico in Italia. Dal Risorgimento alla Seconda Guerra Mondiale, Edizioni Sempre Avanti!, Livorno, 1994

- , Guerra di classe e lotta umana. L'anarchismo in Italia dal Biennio rosso alla Guerra di Spagna (1919-1939), Edizioni Biblioteca Franco Serantini, Pisa, 2001

—, La tradizione dell'anarchismo federato, in L'Unione Anarchica Italiana. Tra rivoluzione europea e reazione fascista (1919-1926), Zero in condotta, Milano, 2006

Emiliani Vittorio, Gli anarchici. Vite di Cafiero, Costa, Malatesta, Cipriani, Gori, Berneri, Borghi, Bompiani, Milano, 1973

Fabbri Luigi, Dittatura e rivoluzione, Libreria editrice internazionale Giovanni Bitelli, Ancona, 1921

—, Epistolario ai corrispondenti italiani ed esteri (1900-1935), a cura di Roberto Giulianelli, Edizioni Biblioteca Franco Serantini, Pisa, 2005

Fedele Santi, Storia della Concentrazione Antifascista, 1927-1934, Feltrinelli, Milano, 1974

— , I repubblicani di fronte al fascismo, 1919-1926, Le Monnier, Firenze, 1983

_ , I repubblicani in esilio nella lotta contro il fascismo (1926-1940), Le Monnier, Firenze, 1989 
—, Una breve illusione: gli anarchici italiani e la Russia sovietica, 19171939, Franco Angeli, Milano, 1996

— , Il retaggio dell'esilio. Saggi sul fuoruscitismo antifascista, Rubbettino, Sovaria Mannelli, 2010

Garosci Aldo, Storia dei fuorusciti, Laterza, Bari, 1953

— , Vita di Carlo Rosselli, Vallecchi, Firenze, 1973

Giovana Mario, Giustizia e Libertà in Italia: storia di una cospirazione antifascista, 1929-1937, Bollati Boringhieri, Torino, 2005

Giulianelli Roberto (a cura di), Luigi Fabbri. Studi e documenti sull'anarchismo tra Otto e Novecento, Edizioni Biblioteca Franco Serantini, Pisa, 2005

Giulietti Fabrizio, Il movimento anarchico italiano nella lotta contro il fascismo, Lacaita, Manduria-Bari-Roma, 2004

- Storia degli anarchici italiani in età giolittiana, Franco Angeli, Milano, 2012

—, Gli anarchici italiani dalla grande guerra al fascismo, Franco Angeli, Milano, 2015

Giustizia e Libertà nella lotta antifascista e nella storia d'Italia. Attualità dei fratelli Rosselli a quaranta anni dal loro sacrificio, La Nuova Italia, Firenze, 1978

Golzio Francesco, Guerra Augusto (a cura di), La cultura italiana del '900 attraverso le riviste, vol. V, "L'Unità», «La Voce Politica» (1915), Einaudi, Torino, 1962

Gozzini Giovanni, Alle origini del comunismo italiano. Storia della federazione giovanile socialista (1907-1921), Dedalo Libri, Bari, 1979

— , La Federazione Giovanile Socialista tra Bordiga e Mussolini (19121914), «Storia contemporanea», XI, $\mathrm{n}^{\circ} 1$, febbraio 1980

Jackson Gabriel, La repubblica spagnola e la guerra civile (1931-1939), Il Saggiatore, Milano, 2003

Kropotkin Pëtr, Sovremennaia nauka $i$ anarkhizm, Russian Free Press, London, 1901, tr. it. Casa Editrice Sociale, Milano, 1922

—, Mutual Aid: a factor of evolution, William Heinemann, London, 1902, tr. it. Casa Editrice Sociale, Milano, 1925

L'Italia in esilio. L'emigrazione italiana in Francia tra le due guerre, Archivio Centrale dello Stato et al., Presidenza del Consiglio dei Ministri - Dipartimento per l'informazione e l'editoria, Roma, 1984

Lanaro Silvio, La cultura antigiolittiana, in Idomeneo Barbadoro (coordinata da), Storia della società italiana, vol. XX, Teti, Milano, 1981 
Larizza Lolli Mirella, Stato e potere nell'anarchismo, in Gian Mario Bravo, Silvia Rota Ghibaudi (a cura di), Il pensiero politico contemporaneo, vol. II, Franco Angeli, Milano, 1986

Le più belle pagine di Carlo Cattaneo, scelte da Gaetano Salvemini, Fratelli Treves, Milano, 1922

Levi Lucio, Il federalismo, in Gian Mario Bravo, Silvia Rota Ghibaudi (a cura di) Il pensiero politico contemporaneo, vol. III, Franco Angeli, Milano, 1986

—, Il pensiero federalista, Laterza, Roma-Bari, 2002

Lotti Luigi, La settimana rossa, Le Monnier, Firenze, 1972

Luti Giorgio, Firenze corpo 8. Scrittori, riviste, editori del '900, Vallecchi, Firenze, 1983

Malandrino Corrado, Socialismo e libertà: autonomie, federalismo, Europa da Rosselli a Silone, Franco Angeli, Milano, 1990

— , Federalismo. Storia, idee, modelli, Carocci, Roma, 1998

- , Autonomia e federalismo: una compresenza necessaria, sussidiaria, sinergica, «Quaderni Fiorentini», XLIII, t. 1, 2014

Manfredonia Gaetano, Gli anarchici italiani in Francia nella lotta antifascista, in La Resistenza sconosciuta. Gli anarchici e la lotta contro il fascismo, Zero in condotta, Milano, 2005

Manganaro Favaretto Gilda, Proudhon in Italia. Una riflessione politica incompresa, Edizioni Università di Trieste, Trieste, 2000

Mannori Luca, 'Autonomia'. Fortuna di un lemma nel vocabolario delle libertà locali tra Francia e Italia, «Quaderni Fiorentini», XLIII, t. 1, 2014

Marshall Peter, Demanding the Impossible. A History of Anarchism, Harper Perennial, London, New York, Toronto and Sydney, 2008

Martini Manuela, La Settimana Rossa, in Mario Isnenghi, Simon Levis Sullam (a cura di), Gli Italiani in guerra. Conflitti, identità, memorie dal Risorgimento ai nostri giorni, vol. II, Le "Tre Italie»: dalla presa di Roma alla Settimana Rossa (1870-1914), Utet, Torino, 2008

Masini Pier Carlo, Gli anarchici italiani tra "interventismo" e "disfattismo rivoluzionario”, «Rivista storica del socialismo», II, n5, gennaio-marzo 1959

- , Gli anarchici italiani e la rivoluzione russa, «Rivista storica del socialismo», V, n¹5-16, giugno-agosto 1962

—, Storia degli anarchici italiani da Bakunin a Malatesta, Rizzoli, Milano, 1974

— , Storia degli anarchici italiani nell'epoca degli attentati, Rizzoli, Milano, 1981 
Mastellone Salvo, Carlo Rosselli e la «rivoluzione liberale del socialismo», Leo S. Olschki, Firenze, 1999

- Storia della democrazia in Europa. Dal XVIII al XX secolo, Utet, Torino, 2006

Molaschi Carlo, Federalismo e libertà, Edizioni Fede!, Roma, 1925

Montanari Fabrizio, Voci del Plata: vita e morte di Torquato Gobbi, Bertani, Verona, 1997

Nacci Michela (a cura di), Figure del liberalsocialismo, Centro Editoriale Toscano, Firenze, 2010

Nettlau Max, Bibliographie de l'anarchie, Burt Franklin, New York, 1968

—, Breve storia dell'anarchismo, Edizioni L'Antistato, Cesena, 1964

Non Mollare (1925). Riproduzione fotografica dei numeri usciti con tre saggi storici di Gaetano Salvemini, Ernesto Rossi, Piero Calamandrei, La Nuova Italia, Firenze, 1955

Paci Deborah, "Proudhon in esilio". La ricezione del pensiero proudhoniano negli ambienti del fuoruscitismo italiano in Francia (anni Venti e Trenta), «Società e Storia», XXXIV, $\mathrm{n}^{\circ}$ 131, gennaio-marzo 2011

Paolini Federico, L'esperienza politica di Oliviero Zuccarini: un repubblicano fra Mazzini, Mill e Sorel, Marsilio, Venezia, 2003

Pecora Gaetano, Socialismo come libertà. La storia lunga di Gaetano Salvemini, Donzelli, Roma, 2012

- , La scuola laica. Gaetano Salvemini contro i clericali, Donzelli, Roma, 2015

Pescosolido Guido, Gaetano Salvemini (1873-1957). Ancora un riferimento. Atti del convegno di studi tenutosi a Roma l'11 e il 12 dicembre 2007 in collaborazione con la Fondazione "Ernesto Rossi e Gaetano Salvemini", Lacaita, Manduria-Bari-Roma, 2010

Petraccone Chiara (a cura di), Federalismo e autonomia in Italia dall'Unità a oggi, Laterza, Roma-Bari, 1995

Prezzolini Giuseppe, Prezzolini alla finestra, Pan Editrice, Milano, 1977

Proudhon Pierre-Joseph, Qu'est-ce que la propriété? Ou Recherche sur le principe du Droit et du Gouvernement, J.-F. Brocard, Paris, 1840, tr. it. Laterza, Bari, 1967

—, Idée générale de la révolution au XIX sièclee, Garnier Frères, Paris, 1851 , tr. it. Centro editoriale toscano, Firenze, 2001

— , Du Principe fédératif et de la nécessité de reconstituer le Parti de la Révolution, E. Dentu, Paris, 1863, tr. it. Mondo Operaio - Edizioni Avanti!, Roma, 1979 
Quasi Annarella, L'antimilitarismo italiano agli inizi del secolo, «Rivista di storia contemporanea», XI, fascicolo 1, gennaio 1982

Ragona Gianfranco, Anarchismo: le idee e il movimento, Laterza, RomaBari, 2013

Ranzato Gabriele, L'eclissi della democrazia: la guerra civile spagnola e le sue origini (1931-1939), Bollati Boringhieri, Torino, 2003

Rerum Scriptor [Gaetano Salvemini], La questione di Napoli (Come si sgominerebbero le camorre amministrative), Edizioni Critica Sociale, Milano, 1901

Riosa Alceo, La «terza via» del «né aderire né sabotare», in Mario Isnenghi, Daniele Ceschin (a cura di), Gli italiani in guerra. Conflitti, identità, memorie dal Risorgimento ai nostri giorni, vol. III, t. 1, La Grande Guerra: dall'Intervento alla «vittoria mutilata», Utet, Torino, 2008

Romaniello Lucia, Le radici del socialismo italiano. Atti del Convegno, Milano, 15-16-17 novembre 1994, Edizioni Comune di Milano «Amici del museo del Risorgimento», Milano, 1997

Romanò Angelo (a cura di), La cultura italiana del '900 attraverso la riviste, vol. III, «La Voce» (1908- 1914), Einaudi, Torino, 1960

Rosselli Carlo, Socialisme libéral, Librairie Valois, Paris, 1930, tr. it. RCS Quotidiani, Milano, 2011

— , Scritti dell'esilio, vol. I, «Giustizia e Libertà» e la Concentrazione antifascista (1929-1934), a cura di Costanzo Casucci, Einaudi, Torino, 1988

- , Scritti dell'esilio, vol. II, Dallo scioglimento della Concentrazione antifascista alla Guerra di Spagna (1934-1937), a cura di Costanzo Casucci, Einaudi, Torino, 1992

Rosselli Nello, Saggi sul Risorgimento e altri scritti, Einaudi, Torino, 1946

Rossi Ernesto, Un democratico ribelle. Cospirazione antifascista, carcere, confino. Scritti e testimonianze, a cura di Giuseppe Armani, Guanda, Parma, 1975

Rota Ghibaudi Silvia, Pierre-Joseph Proudhon, in Gian Mario Bravo, Silvia Rota Ghibaudi (a cura di), Il pensiero politico contemporaneo, vol. II, Franco Angeli, Milano, 1986

Rotelli Ettore, L'eclissi del federalismo. Da Cattaneo al Partito d'Azione, Il Mulino, Bologna, 2003

Roveri Alessandro, Salvemini, le grandi riforme e i contadini meridionali, in Idomeneo Barabdoro (coordinata da), Storia della società italiana, vol. XX, Teti, Milano, 1981

Sabbatucci Giovanni (a cura di), Storia del socialismo italiano, vol. II, L'età giolittiana (1900-1914), Il Poligono, Roma, 1980 
Sacchetti Giorgio, Compagni di strada. Gli anarchici italiani e la questione delle alleanze (1914-1944), in L'Unione Anarchica Italiana. Tra rivoluzione europea e reazione fascista (1919-1926), Zero in condotta, Milano, 2006

Salvadori Massimo L., Gaetano Salvemini, Einaudi, Torino, 1963

Salvemini Gaetano, La rivoluzione francese (1788-1792), Pallestrini \& C., Milano, 1905

—, Il ministro della malavita: notizie e documenti sulle elezioni giolittiane nell'Italia meridionale, Edizioni della Voce, Firenze, 1910

—, Mazzini, Edizioni La Voce, Roma, 1920

- , Opere II, Scritti di storia moderna e contemporanea, vol. II, Scritti sul Risorgimento, Feltrinelli, Milano, 1961

- , Opere IV, Il Mezzogiorno e la democrazia italiana, vol. I, Il ministro della mala vita e altri scritti sull'Italia giolittiana, Feltrinelli, Milano, 1962

- , Opere IV, Il Mezzogiorno e la democrazia italiana, vol. II, Movimento socialista e questione meridionale, Feltrinelli, Milano, 1963

- , Opere VIII, Scritti vari (1900-1957), Feltrinelli, Milano, 1978

—, Opere IX, Carteggi, vol. I, (1895-1911), Feltrinelli, Milano, 1968

Schirone Franco (a cura di), Cronache anarchiche. Il giornale Umanità Nova nell'Italia del Novecento (1920-1945), Zero in condotta, Milano, 2010

Scrima Valerio A., Vaccaro Salvo, Conversazione su anarchia $e$ autogoverno, La Fiaccola, Ragusa, 1994

Senta Antonio, Note su Torquato Gobbi, un anarchico problematico, «Clio», $\mathrm{XLIV}, \mathrm{n}^{\circ}$ 3, settembre-ottobre 2008

_ , Utopia e azione, per una storia dell'anarchismo in Italia (1848-1984), Elèuthera, Milano, 2016

Senta Antonio (a cura di), La rivoluzione scende in strada: la settimana rossa nella storia d'Italia, 1914-2014. Atti del convegno organizzato dall'Archivio storico della Federazione anarchica italiana, Imola, sabato 27 settembre 2014, Zero in condotta, Milano, 2016

Severini Marco (a cura di), La settimana rossa, a cura di Marco Severini, Aracne, Roma, 2014

Signori Elisa, Tesoro Marina, Il verde e il rosso: Fernando Schiavetti e gli antifascisti nell'esilio fra repubblicanesimo e socialismo, Le Monnier, Firenze, 1987

Spadolini Giovanni, I repubblicani dopo l'Unità. Quinta edizione accresciuta con una parte aggiuntiva sul PRI dalla sua costituzione al 1984, Le Monnier, Firenze, 1984 
Spriano Paolo, Torino operaia nella Grande Guerra, Einaudi, Torino, 1960 Tabor Davide, L'arte della propaganda. Il modello di proselitismo del Psi tra fine Ottocento e inizio Novecento, «Contemporanea», XIV, $\mathrm{n}^{\circ} 4$, 2011

Tarizzo Domenico, L'anarchia: storia dei movimenti libertari nel mondo, Mondadori, Milano, 1973

Tesoro Marina, Democrazia in azione: il progetto repubblicano da Ghisleri a Zuccarini, Franco Angeli, Milano, 1996

Thomas Hugh, Storia della guerra civile spagnola, Einaudi, Torino, 1963

Torcellan Nada, Gli italiani in Spagna: bibliografia della guerra civile spagnola, Franco Angeli, Milano, 1988

Tombaccini Simonetta, Storia dei fuorusciti italiani in Francia, Mursia, Milano, 1988

Uno della Tribù [Rodolfo Vella], Preanarchia: pareri pratici sull'organizzazione della società preanarchica, Edizioni Vogliamo, Lugano, 1932

Vaccaro Gennaro (a cura di), Panorama biografico degli italiani d'oggi, vol. I, Curcio, Roma, 1956

Valiani Leo, Il Partito socialista italiano nel periodo della neutralità, 19141915, Feltrinelli, Milano, 1963

Venza Claudio, Tra rivoluzione e guerra. Libertari italiani nella Spagna degli anni Trenta, in La Resistenza sconosciuta. Gli anarchici e la lotta contro il fascismo, Zero in condotta, Milano, 2005

— , Anarchia e potere nella guerra civile spagnola (1936-1939), Elèuthera, Milano, 2009

Vitali Stefano (a cura di), Istituto Storico della Resistenza in Toscana, Archivio Gaetano Salvemini, I, Manoscritti e materiali di lavoro, Ministero per i Beni Culturali e Ambientali - Ufficio Centrale per i Beni Archivistici, Roma, 1998

Vivarelli Roberto, Storia delle origini del fascismo: l'Italia dalla grande guerra alla marcia su Roma, vol. I, Il Mulino, Bologna, 1991

Ward Colin, Anarchia come organizzazione. La pratica della libertà, Elèuthera, Milano, 2006

Woodcock George, L'Anarchia. Storia delle idee e dei movimenti libertari, Feltrinelli, Milano, 1966

Wyatt Chris, A recipe for a cookshop of the future: G.D.H. Cole and the conundrum of sovereignty, "Capital \& Class», 30, Autumn 2006

Zani Luciano, Italia Libera. Il primo movimento antifascista clandestino (1923-1925), Laterza, Roma-Bari, 1975 


\section{Fonti emerografiche}

Ansaldi Carlo Francesco, Repubblicanesimo sociale e anarchismo, «Umanità Nova», III 7 aprile 1922, segue nota di Errico Malatesta

- , Repubblicanesimo sociale e anarchismo. Consensi e dissensi sulla teoria e la tecnica della rivoluzione, "Umanità Nova», III, 14 aprile 1922, segue nota di Errico Malatesta

—, Repubblicanesimo sociale e anarchismo, «Umanità Nova», III, 26 aprile 1922

—, Repubblicanesimo sociale e anarchismo, «Umanità Nova», III, 29 aprile 1922, segue nota di Errico Malatesta

[L'Avanguardia], Dalla giovine Italia socialista - Dall'Italia settentrionale, «L'Avanguardia», VIII, 19 luglio 1914

— , Dalla giovine Italia socialista - Federaz. Provinciale Reggiana, «L'Avanguardia», VIII, 27 settembre 1914

— , Impazienza, «L'Avanguardia», IX, 14 novembre 1915

Consiglio Umberto, Gli anarchici e "G. e L.", "Giustizia e Libertà», II, 22 novembre 1935, segue Risposta di G.L. [Carlo Rosselli]

Damiani Gigi [Damiani Luigi], Prima di ricominciare...per ricominciare, «Umanità Nova», III, 2 e 9 settembre 1922

— , Revisione, «Pagine Libertarie», II, 20 ottobre 1922, segue Postilla di Charles L'Ermite [Carlo Molaschi]

—, Anarchismo e federalismo - Le conclusioni di Gigi Damiani, «Pagine Libertarie», II, 20 novembre 1922

G. D. [Damiani Luigi], Il nostro programma. Parole che interessano amici e nemici, «Fede!», I, 16 settembre 1923

Fabbri Luigi, I repubblicani e noi, «Volontà», I, 16 giugno 1919

—, Ritorno al sindacalismo?, «Studi Sociali», II, 12 giugno 1931

— , Qualche risposta all'inchiesta di «G.L.», «Quaderni di Giustizia e Libertà», $n^{\circ}$ 7, giugno 1933

[Fienga Dino], Repetitia iuvant, «Il Grido», II, 5 gennaio 1918

Il Grido, Emeroteca, «Il Grido», I, 5 novembre 1917

—, Schiarimenti. Per intenderci, «Il Grido», I, 20 novembre 1917

—, Emeroteca, «Il Grido», I, 20 dicembre 1917

Klein Giovan Battista, La Costituente, «L'Unità», VIII, 18 gennaio 1919

Leonetti Alfonso, L'unicità del mezzo, «Il Grido», I, 5 dicembre 1917

Longobardi Ernesto Cesare, Le confessioni di un anglofilo, «L'Avanguardia», VIII, 22 novembre 1914 
M. S. [Levi Carlo, Ginzburg Leone], Il concetto di autonomia nel programma di "G.L.", «Quaderni di Giustizia e Libertà», $\mathrm{n}^{\circ} 4$, settembre 1932

Malatesta Errico, Noi ed i repubblicani, «Umanità Nova», I, 25 aprile 1920

—, Noi ed i mazziniani, «Umanità Nova», I, 9 maggio 1920

—, Ancora sulla repubblica, «Umanità Nova», I, 21 maggio 1920

—, Che cos'è la repubblica sociale?, «Umanità Nova», III, 2 aprile 1922

— , Repubblicanesimo sociale e anarchismo, «Umanità Nova», III, 27 aprile 1922

—, Repubblica e rivoluzione, «Pensiero e Volontà», I, 1 giugno 1924

— , Ancora di repubblica e rivoluzione, «Pensiero e Volontà», I, 15 giugno 1924

—, A proposito di costituente, «Pensiero e Volontà», I, 15 ottobre 1924

Mauri E., Federalismo e socialismo, «L'Avanguardia», IX, 8 agosto 1915

Meschi Alberto, Gli anarchici e "Giustizia e Libertà", "Il Martello», XVII, 2 aprile 1932

Charles L'Ermite [Molaschi Carlo], Sulla situazione (pensieri e commenti), «Pagine Libertarie», II, 21 settembre 1922

Molaschi Carlo, Anarchismo e federalismo - Postilla del compilatore, «Pagine Libertarie», II, 20 novembre 1922

—, Dalla teoria alla realtà, «Fede!», II, 17 agosto 1924

—, Le idee federaliste nell'attuale crisi italiana, «Pensiero e Volontà», I, 15 ottobre 1924

—, Il posto degli anarchici nell'attuale crisi italiana, «Pensiero e Volontà», I, 1 novembre 1924

Noi, Linee programmatiche, «La Critica Politica», I, 25 dicembre 1920-1 gennaio 1921

[Rosselli Carlo, Garosci Aldo], "Libertà e Giustizia" e "Giustizia e Libertà", "Quaderni di Giustizia e Libertà», n 2, marzo 1932

G. L. [Rosselli Carlo], «Giustizia e Libertà», I, Contro lo Stato, 21 settembre 1934

M. S. [Sartin Max], Sovietismo, «L'Adunata dei Refrattari», XI, 17 settembre 1932

Un insegnante, La Costituente, «L'Unità», VIII, 25 gennaio 1919, segue Postilla de l'Unità [G. Salvemini]

Un Travet [Salvemini Gaetano], Le origini della reazione, «Critica Sociale», VIII, 1 luglio e 1 agosto 1899

Rerum Scriptor [Salvemini Gaetano], La questione di Napoli, «Critica Sociale», X, 1 e 16 dicembre 1900 
Salvemini Gaetano, L'astrattismo del concretismo, «L'Unità», II, 31 gennaio 1913

—, Il nostro concretismo, «L'Unità», II, 28 febbraio 1913

—, Alla ricerca di una formula, «L'Unità», II, 7, 14 e 21 marzo 1913

—, Quel che l'Unità non può dare e quel che non vuol fare, «L'Unità», II, 2 maggio 1913

—, La Costituente, «L'Unità», VIII, 2 gennaio 1919

— , La provincia di Bari nel 1920, «L'Unità», IX, 16 settembre 1920

Giesse [Salvemini, Gaetano], Federalismo superato, «Giustizia e Libertà», III, 10 gennaio 1936

Savelli Rodolfo, Alla ricerca di una formula. Che cosa vuole l'«Unità»?, «L'Unità», II, 7 marzo 1913

Zagari Guglielmo, Polemiche sul metodo, con Postilla di Gaetano Salvemini, «L'Unità», II, 25 aprile 1913

Zuccarini Oliviero, Tendenze e fini di un movimento autonomista, «La Critica Politica», II, 25 aprile 1922

—, Qualche idea sull'ordinamento dello Stato, «La Critica Politica», IV, 25 luglio 1924

\section{Fonti digitali}

Archivio «Famiglia Berneri-Aurelio Chessa», a cura della Biblioteca Panizzi di Reggio Emilia

$<\underline{\text { http://panizzi.comune.re.it/allegati/ABC completo.pdf }>}$ (09/2019)

Tuccillo Fulvio, Il caso Fienga: le testimonianze di una vita straordinaria $<$ http://www.bnnonline.it/index.php?it/158/il-caso-fienga-letestimonianze-di-una-vita-straordinaria $>$ (09/2019)

Vallentyne Peter, Libertarianism, in The Stanford Encyclopedia of Philosophy, Edward N. Zalta, Spring 2012 Edition $<$ http://plato.stanford.edu/archives/spr2012/entries/libertarianism $>$ $(09 / 2019)$ 


\section{Indice dei nomi}

Acciai E. 179n.; 214

Adamo P. 11n.; 15; 17; 27n.; 35n.; 72n.; 7375; 77; 78n; 95n.; 112; 115; 134n.; 136n.; 163; 176n.; 211

Albanese G. 214

Albasini Scrostati V. 111

Aldrighi C. 158 n.

Ambrosoli L. 214

Andreucci F. 29n.; 32n.; 47n.; 214

Angelini G. 79n.; 164; 165n; 214

Angioletti F. 33

Ansaldi C. F. 91-93; 224

Antonioli M. 12n.; 34n.; 36n.; 94n.; 143n.; 149n.; 150n.; 178n.; 214

Arfé G. 26-27n.; 29n.; 31n.; 214

Armani G. 221

Aruffo A. 214

Ascarelli T. 111

Ascoli M. 111

Asor Rosa A. 51n.; 52; 53n.; 214

Bagnoli P. 214

Bakunin M. A. 23n.; 72; 85; 95-96; 98; 104; $116 ; 121 ; 141 ; 158 ; 160 ; 184 ; 230$

Barbadoro I. 36n.; 52n.; 55n.; 63n; 215; 217; 218

Battistelli L. 16n.; 39; 137

Battisti C. 33

Battisti L. 110

Bauer R. 111

Bellet G. 40

Bennassar B. 13n.; 215

Berneri C. 11-19; 23-86; 88-93; 95-111; $117 \mathrm{n} ; 119-156 ; 160-161 ; 163-177 ; 179-$ $192 ; 194-206 ; 207-213$

Berneri M. L. 37; 42

Bertani A. 175

Berti G. 14-16; 19; 23n.; 26n.; 51n.; 70n.; 93n.; 116n.; 164n.; 179n.; 211; 215

Bertolini P. 82
Bertolucci F. 45n.; 215

Bettini L. 215

Bettini (Schettini) S. 128; 138

Bezza B. 122n.; 216

Biagianti I. 57n.; 80n

Bianciardi S. 26n.; 215

Blanqui A. 28; 32-33

Bravo G. M. 219; 221

Bocci E. 110

Boccolari G. 27n.; 211; 215

Bonaparte N. 48

Bordiga A. 29

Bosicchi M. 33

Bovio G. 46n.; 67; 89; 184

Bracci L. 111

Bresciani M. 141n.; 215

Calamandrei P. $13 ; 85 ; 90$

Caleffi G. 11; 42; 73; 114n.; 136n.; 165

Calò G. 49n.; 68n.

Campos Boralevi L. 215

Cafiero C. 179n.; 180; 184

Cagliostro A. 48

Capetta F. 59n.; 215

Caretti S. 31n.; 215

Carrozza G. 14; 31n.; 43n.; 58n.; 70; 155; $171 ; 212$

Casali L. 27n.; 30n.; 211; 215

Casucci C. 179n.; 221

Cattaneo C. 16; 46n.; 80-81; 86; 88-89; 95$96 ; 121 ; 126-128 ; 130 ; 137 ; 139-140$; $175 ; 184 ; 198-200$

Cecchini L. 89n.; 216

Cerasi L. 51n.; 216

Cerrito G. 14; 15n.; 36n.; 80n.; 116n.; 122n.; $212 ; 216$

Ceschin D. 31n.; 221

Ceva L. 13n.; 216

Ceva U. 110 
Chessa A. 11; 14; 39n.; 54n.; 137n.; 161; 165; 173n.; 174n.; 211; 216

Chessa F. 165

Chiaramonte U. 30n.; 53n.; 87n.; 88n.; 89n.; 91n.; 102n.; 216

Chiodini A. 140

Chomsky N. 20; 216

Cingari G. 56n.; 57n.; 80n.; 190n.; 216

Ciuffoletti Z. 31n.; 86n.; 216cl

Clemenceau G. B. 65

Cole G. D. H. 22n.

Colombo A. 86n.; 216

Colorni E. 110

Considerant V. P. 58

Consiglio U. 177-178; 183; 224

Cortesi L. 31n.; 216

Crispi F. 82

D'Errico S. 12n.; 13n.; 14n.; 15; 17; 28n.; 100n.; 116n.; 119n.; 163n.; 164n.; 179n.; 212

De Agostini M. 73n.

De Caro G. 55n.; 217

De Marco L. 36n.; 217

De Maria C. 12n.; 13n.; 15; 25n.; 27n.; 41n.; 73n.; 77; 78n.; 112n.; 115; 116n.; 120n.; 128n.; 171; 175n.; 176n.; 182n.; 191; $201 ; 212$

Degl'Innocenti M. 26n.; 29n.; 30n.; 31n.; 35n.; 57n.; 80n.; 166n.; 190n.; 215-217

Della Peruta F. 79n.; 217

Dadà A. 36n.; 217

Damiani L. 73; 94-96; 98; 221

Damiani M. 110

Detti T. 29n.; 32n.; 47n.; 214

Di Lembo L. 14n.; 15n.; 21-22; 42n.; 52n.; 63n.; 73n.; 86n.; 102n.; 122n.; 155n.; $211 ; 217$

Einaudi L. 175

Emiliani V. 120n.; 217

Fabbri L. $67 ; 73 ; 135-136 ; 154 ; 156-159$; $160 ; 180 ; 184 ; 217 ; 224$

Farini L. C. 82

Fedele S. 45n.; 87n.; 88; 93n.; 120; 122n.; 129n.; 138n.; 141n.; 157n.; 160n.; 179n.; 217

Feri P. 14n.; 42n.; 52n.; 86n.; 155n.; 211
Ferrari G. 46n.; 79n.; 80-81; 88-89; 95-96; $102 ; 121 ; 128 ; 139 ; 184$

Ferraris C. F. 82

Fienga B. 45-46; 66-67; 224

Finzi R. 30n.; 215

Foa V. 157

Fochi A. 12; 25; 29n.; 33n.; 35n.; 41; 85n.; 86; 110n.; 111n.; 212

Franqueville A. 74

Friscia S. 28

Furlotti G. 27n.

Garosci A. 158n.; 218; 225

Gervasoni M. 29n.; 212

Ghisleri A. 88-90

Gille P. 180

Ginzburg L. 110; 157; 186; 225

Giovana M. 141n.; 218

Giua R. 157

Giulianelli R. 158n.; 217-218

Giulietti, Fabrizio 36n.; 42n.; 45n.; 63n.; 122n.; 160n.; 192; 218

Gobbi T. 34-36; 42

Gobetti P. 13; 91; 110; 139; 175

Godwin W. 180

Golzio F. 55n.; 218

Gozzini G. 29n.; 31n.; 32n.; 218

Grassi F. 56n.; 190n.

Grassi Orsini F. 57n.; 190n.

Guerra A. 55n.; 218

Guidi F. 163n.; 212

Gurvitch G. 158

Isnenghi M. 31n.; 214; 219; 221

Jacini S. F. 82

Jackson G. 203n.; 218

Jacometti A. 128

Jahier P. 85; 86n.

Kant I. 48

Kerenskij A. F. 44

Klein G. B. 147; 224

Kropotkin P. A. 46n.; 70; 95; 104-110; 181; $199 ; 210 ; 218$

Lacaita C. 57n.; 80n.; 166n.; 188n.

Lanaro S. 52n.; 218

Larizza Lolli M. 219

Lenin V. I. 44;48-49; 82-83 
Leonetti A. $47 ; 65 ; 224$

Leverdays, É. 58

Levi C. 157; 180; 225

Levi L. 219

Levis Sullam S. 214; 219

Longobardi E. C. 32; 224

Lotti L. 35n.; 219

Lucchini L. 82

Lussu E. 141

Luti G. 51n.; 219

Machiavelli N. 52

Madrid Santos F. 12n.; 14; 28n.; 53n.; 86n.; 212

Malandrino C. 23n.; 87n.; 88; 102; 157n.; 179n.; 188n.; 219

Malatesta, E. $13 ; 73 ; 84 ; 91-93 ; 101 ; 139$; 145; 155n.; 180-181; 184; 224-225

Manci G. 110

Manfredonia G. 122n.; 128n.; 160n.; 219

Manganaro Favaretto G. 79n.; 158n.; 219

Mannori L. 219

Marshall P. 22n.; 219

Martini M. 219

Marx K. 28; 49; 158

Marzocchi U. 14

Masaniello (Aniello) T. 48

Masetti A. 36

Masini P. C. 14; 27n.; 33n.; 35n.; 36n.; 39; 45n.; 46n.; 54n.; 57n.; 115; 134n.; 137n.; 211-212; 214; 219

Mastellone S. 60n.; 220

Mauti P. 16n.; 57n.; 81n.; 120n.; 139n.; 211

Mazzini G. 27; 47; 85-86; 89; 96; 128; 141; $160 ; 175 ; 213$

Melli G. 49n.

Meneghetti E. 111

Merlino F. S. 107; 180-181

Meschi A. 143-144; 225

Minghetti M. 82

Mink P. 28

Molaschi C. 94-96; 98; 101; 102n.; 103n.; 220; 224-225

Moneta V. 111

Montanari F. 27n.; 33n.; 34n.; 39; 212; 220

Morra di Lavriano U. 111

Mussolini B. 32

Nacci M. 179n.; 211; 220

Nettlau, Max 22n.; 96; 175; 220

Nietzsche F. 48
Nitti F. 141

Paci D. 158n.; 220

Paolini F. 87n.; 220

Papini G. 52-53

Papini M. 84n.

Pareto V. 175

Parri F. 111

Pechar S. W. 14n.; 212

Pecora G. 73n.; 74n.; 220

Pescosolido G. 57n.; 188n.; 220

Pestalozzi J. H. 29

Piccolo S. 59n.; 215

Pilati G. 110

Pisacane C. 46n.; 67; 79n.; 95; 102; 116; $136 ; 140 ; 160 ; 179$ n.; 184

Prampolini C. 26n.; 27n.; 29; 34-35; 38-39

Prezzolini G. 52-53; 220

Proudhon P.-J. 23n.; 60-61; 79n.; 86; 89; $100 ; 104 ; 116 ; 136 ; 140 ; 158 ; 166$; 179n.; 180-181; 185-186; 220

Quasi A. 31n.; 221

Ragona G. 221

Ranzato G. 13n.; 203n.; 221

Rasputin G. E. 48

Reclus J. É. 95

Riosa A. 31n.; 221

Rittinghausen M. 58; 60

Robespierre M. 48

Romaniello L. 26n.; 221

Romanò A. 53n.; 221

Rosa G. 184

Rosselli A. 86

Rosselli C. 13; 18; 23n.; 86; 110-111; 122n.; 141; 157-160; 178-182; 183n.; 184-188; 189n.; 191-192; 196; 213; 221; 224-225

Rosselli N. 13; 84-86; 110-111; 175; 221

Rossetti R. 138

Rossi E. 13; 85; 110-111; 221

Rota Ghibaudi S. 20n.; 219; 221

Rousseau J.-J. 58-60; 80

Roveri A. 55n.; 221

Sabbatucci G. 35n.; 216; 221

Sacchetti G. 15n.; 16; 17n.; 26; 36n.; 42n.; 51; 70n.; 164n.; 179n.; 211; 222

Salvadori M. L. 55n.; 222

Salvemini G. 12-13; 16; 18; 53-60; 62; 66; 68n.; 70; 73n.; 74-76; 77n.; 78; 80-81; 
84-86; 88; 91; 94; 97; 103-104; 106; $108 ; 110-111 ; 116 ; 124 ; 126-127 ; 129$; $137 ; 139 ; 146 ; 160 ; 166 ; 183-184 ; 189-$ $191 ; 195 ; 198 ; 200 ; 206 ; 212-213 ; 221-$ $222 ; 225-226$

Savelli R. 57n.; 226

Scaliati G. 73n.

Scavino M. 91n.; 213

Schiavetti F. 87; 138-141; 160

Schiavina R. (Max Sartin) 150-154; 194; $197 ; 210-211 ; 225$

Schirone F. 73n.; 140n.; 222

Scrima V. A. 20n.; 21; 222

Senta A. 34n.; 35n.; 36n.; 84n.; 222

Severini M. 35n.; 222

Signori E. 87n.; 138n.; 160n.; 222

Simon J. 75

Simonini A. 28

Sorel G. 180

Sorti A. 14; 35n.; 57n.; 115; 134n.; 211

Spadolini G. 222

Spriano P. 29n.; 223

Stirner M. 48

Strambi C. 15; 27n.; 35; 53; 54n.; 57n.; 63 n.; 70n.; 213

Tabor D. 26n.; 223

Tarquandi N. 111

Tasca A. 29; 39.; 213

Tcherkesof V. 180

Tesoro M. 87n.; 88n.; 138n.; 141n.; 160n.; 222-223
Thomas H. 13n.; 203n.; 223

Tocqueville A. 74; 129; 140

Tombaccini S. 120n.; 223

Torcellan N. 13n.; 223

Tuccillo F. 45n.; 226

Vaccaro G. 45n.; 223

Vaccaro S. 20-21; 222

Vallentyne P. 22n.; 226

Vannucci D. 110-111

Vella R. 149; 223

Venza C. 14n.; 203n.; 223

Vitali S. 86n.; 223

Vittorio Emanuele III di Savoia 111

Vivarelli R. 63n.; 66n.; 223

Volterra F. 140

Wallace A. R. 28

Ward C. 20; 21n.; 223

Woodcock G. 19-20; 22n.; 223

Wyatt C. 22n.; 223

Zagari G. 57n.; 226

Zani L. 110n.; 223

Zani R. 36n.

Zanotti-Bianco U. 111

Zibordi G. 33

Zuccarini O. 87-88; 90-91; 96; 127; 139; 166n.; 184; 200; 226 
ANNO 2007

Bracardi M., La Materia e lo Spirito. Mario Ridolfi nel paesaggio umbro

Coppi E., Purines as Transmitter Molecules. Electrophysiological Studies on Purinergic Signalling in Different Cell Systems

Mannini M., Molecular Magnetic Materials on Solid Surfaces

Natali I., The Ur-Portrait. Stephen Hero ed il processo di creazione artistica in A Portrait of the Artist as a Young Man

Petretto L., Imprenditore ed Università nello start-up di impresa. Ruoli e relazioni critiche

ANNO 2008

Bemporad F., Folding and Aggregation Studies in the Acylphosphatase-Like Family

Buono A., Esercito, istituzioni, territorio. Alloggiamenti militari e «case Herme» nello Stato di Milano (secoli XVI e XVII)

Castenasi S., La finanza di progetto tra interesse pubblico e interessi privati

Colica G., Use of Microorganisms in the Removal of Pollutants from the Wastewater

Gabbiani C., Proteins as Possible Targets for Antitumor Metal Complexes: Biophysical Studies of their Interactions

ANNO 2009

Decorosi F., Studio di ceppi batterici per il biorisanamento di suoli contaminati da Cr(VI)

Di Carlo P., I Kalasha del Hindu Kush: ricerche linguistiche e antropologiche

Di Patti F., Finite-Size Effects in Stochastic Models of Population Dynamics: Applications to Biomedicine and Biology

Inzitari M., Determinants of Mobility Disability in Older Adults: Evidence from Population-Based Epidemiologic Studies

Macrì F., Verso un nuovo diritto penale sessuale. Diritto vivente, diritto comparato e prospettive di riforma della disciplina dei reati sessuali in Italia

Pace R., Identità e diritti delle donne. Per una cittadinanza di genere nella formazione

Vignolini S., Sub-Wavelength Probing and Modification of Complex Photonic Structures

ANNO 2010

Fedi M., "Tuo lumine». L'accademia dei Risvegliati e lo spettacolo a Pistoia tra Sei e Settecento

Fondi M., Bioinformatics of genome evolution: from ancestral to modern metabolism. Phylogenomics and comparative genomics to understand microbial evolution

Marino E., An Integrated Nonlinear Wind-Waves Model for Offshore Wind Turbines

Orsi V., Crisi e Rigenerazione nella valle dell'Alto Khabur (Siria). La produzione ceramica nel passaggio dal Bronzo Antico al Bronzo Medio

Polito C., Molecular imaging in Parkinson's disease

Romano R., Smart Skin Envelope. Integrazione architettonica di tecnologie dinamiche e innovative per il risparmio energetico

ANNO 2011

Acciaioli S., Il trompe-l' $e i l$ letterario, ovvero il sorriso ironico nell'opera di Wilhelm Hauff

Bernacchioni C., Sfingolipidi bioattivi e loro ruolo nell'azione biologica di fattori di crescita e citochine

Fabbri N., Bragg spectroscopy of quantum gases: Exploring physics in one dimension

Gordillo Hervás R., La construcción religiosa de la Hélade imperial: El Panhelenion

Mugelli C., Indipendenza e professionalità del giudice in Cina

Pollastri S., Il ruolo di TAF12B e UVR3 nel ciclo circadiano dei vegetali

Salizzoni E., Paesaggi Protetti. Laboratori di sperimentazione per il paesaggio costiero euro-mediterraneo 
ANNO 2012

Evangelisti E., Structural and functional aspects of membranes: the involvement of lipid rafts in Alzheimer's disease pathogenesis. The interplay between protein oligomers and plasma membrane physicochemical features in determining cytotoxicity

Bondì D., Filosofia e storiografia nel dibattito anglo-americano sulla svolta linguistica

Petrucci F., Petri Candidi Decembrii Epistolarum iuvenilium libri octo. A cura di Federico Petrucci

Alberti M., La 'scoperta' dei disoccupati. Alle origini dell'indagine statistica sulla disoccupazione nell'Italia liberale (1893-1915)

Gualdani R., Using the Patch-Clamp technique to shed light on ion channels structure, function and pharmacology

Adessi A., Hydrogen production using Purple Non-Sulfur Bacteria (PNSB) cultivated under natural or artificial light conditions with synthetic or fermentation derived substrates

Ramalli A., Development of novel ultrasound techniques for imaging and elastography. From simulation to real-time implementation

ANNO 2013

Lunghi C., Early cross-modal interactions and adult human visual cortical plasticity revealed by binocular rivalry

Brancasi I., Architettura e illuminismo: filosofia e progetti di città nel tardo Settecento francese

Cucinotta E., Produzione poetica e storia nella prassi e nella teoria greca di età classica

Pellegrini L., Circostanze del reato: trasformazioni in atto e prospettive di riforma

Locatelli M., Mid infrared digital holography and terahertz imaging

Muniz Miranda F., Modelling of spectroscipic and structural properties using molecular dynamics

Bacci M., Dinamica molecolare e modelli al continuo per il trasporto di molecole proteiche - Coarsegrained molecular dynamics and continuum models for the transport of protein molecole

Martelli R., Characteristics of raw and cooked fillets in species of actual and potential interest for italian aquaculture: rainbow trout (oncorhynchus mykiss) and meagre (argyrosomus regius)

ANNO 2014

Lana D., A study on cholinergic signal transduction pathways involved in short term and long term memory formation in the rat hippocampus. Molecular and cellular alterations underlying memory impairments in animal models of neurodegeneration

Lopez Garcia A., Los Auditoria de Roma y el Athenaeum de Adriano

Pastorelli G., L'immagine del cane in Franz Kafka

Bussoletti A., L'età berlusconiana. Il centro-destra dai poli alla Casa della Libertà 1994-2001

Malavolti L., Single molecule magnets sublimated on conducting and magnetic substrates

Belingardi C., Comunanze urbane. Autogestione e cura dei luoghi

Guzzo E., Il tempio nel tempio. Il tombeau di Rousseau al Panthéon di Parigi

ANNO 2015

Lombardi N., MEREAFaPS: uno Studio di Farmacovigilanza Attiva e Farmacoepidemiologia in Pronto Soccorso

Baratta L., "A Marvellous and Strange Event». Racconti di nascite mostruose nell'Inghilterra della prima età moderna

Richichi I.A., La teocrazia: crisi e trasformazione di un modello politico nell'Europa del XVIII secolo

Palandri L., I giudici e l'arte. Stati Uniti ed Europa a confronto

Caselli N., Imaging and engineering optical localized modes at the nano scale

Calabrese G., Study and design of topologies and components for high power density dc-dc converters

Porzilli S., Rilevare l'architettura in legno. Protocolli metodologici per la documentazione delle architetture tradizionali lignee: $i$ casi studio dei villaggi careliani in Russia 
ANNO 2016

Martinelli S., Study of intracellular signaling pathways in Chronic Myeloproliferative Neoplasms

Abbado E., "La celeste guida”. L'oratorio musicale a Firenze: 1632-1799

Focarile P., I Mannelli di Firenze. Storia mecenatismo e identità di una famiglia fra cultura mercantile e cultura cortigiana

Nucciotti A., La dimensione normativa dell'imprenditorialità accademica. Tre casi di studio sugli investigatori principali, i loro gruppi di ricerca e i fattori di innesco dell'imprenditorialità accademica

Peruzzi P., La inutilizzabilità della prestazione

Lottini E., Magnetic Nanostructures: a promising approach towards RE-free permanent magnets

Uricchio T., Image Understanding by Socializing the Semantic Gap

ANNO 2017

Valenti R., Cerebral Small Vessel Disease and Cerebral Amyloid Angiopathy: neuroimaging markers, cognitive features and rehabilitative issues

Starnini M., L'uomo tutto intero. Biografia di Carlo Livi, psichiatra dell'Ottocento

Verardi D., La scienza e i segreti della natura a Napoli nel Rinascimento: la magia naturale di Giovan Battista Della Porta

Minicucci G., Il dolo nella bancarotta. Alla ricerca della tipicità soggettiva della fattispecie patrimoniale

Pattelli L., Imaging light transport at the femtosecond scale: a walk on the wild side of diffusion

Egea Molines M.T., Etnobotánica en el Alto Valle del Reno (Toscana y Emilia-Romaña, Italia). Etnobotanica nell'Alta Valle del Reno (Toscana ed Emilia-Romagna, Italia)

Romano I.M., Pressione turistica sul Centro Storico di Firenze - sito UNESCO. Un modello per la valutazione dell'impatto percettivo

ANNO 2018

Costa A., Histaminergic neurotransmission as a gateway for the effects of the fat sensing molecule Oleoylethanolamide. Focus on cognition and stress-reactivity

Solera D., "Sotto l'ombra della patente del Santo Officio». I familiares dell'Inquisizione romana tra XVI e XVII secolo

Landi G., Secession and Referendum. A new Dimension of International Law on Territorial Changes?

Sacchetti A., La costituente libertaria di Camillo Berneri. Un disegno politico tra federalismo e anarchismo

Livi L.F., New quantum simulations with ultracold Ytterbium gases

Bellini E., Ambienti sensoriali "terapeutici” che rendano Abili. Un progetto integrato di vita per persone con Disturbi dello Spettro Autistico

Piscitelli L.R., Serviceability and post-failure behaviour of laminated glass structural elements 
